\title{
Constraining Standard Model and Beyond Standard Model Higgs boson couplings in the four lepton decay channel with the ATLAS detector
}

by

\section{David Di Valentino}

\author{
A thesis submitted to the \\ Faculty of Graduate and Postdoctoral Affairs \\ in partial fulfillment of the requirements \\ for the degree of \\ Doctor of Philosophy \\ in the subject of \\ Particle Physics \\ Department of Physics \\ Carleton University \\ Ottawa-Carleton Institute for Physics \\ Ottawa, Ontario \\ April, 2018
}

(C) David Di Valentino, 2018 


\section{Abstract}

In 2012, the Standard Model of Particle Physics was "completed" with the discovery of a new particle consistent with a Standard Model Higgs boson. While this discovery marks a monumental achievement for particle physics, there are still several gaps in our understanding of physics which the Standard Model cannot explain. Therefore, we necessarily enter a phase beyond discovery where the properties of the Higgs boson must be understood with increasing detail, including deviations from its expected charge-parity $(\mathcal{C P})$ even nature, and couplings to other particles. Higgs boson decays to four leptons $\left(H \rightarrow Z Z^{*} \rightarrow 4 \ell\right)$ provide an ideal avenue for property measurements, due to their high signal-to-background ratio, and the high precision of electron and muon measurements in ATLAS.

This thesis presents an analysis which selects $H \rightarrow Z Z^{*} \rightarrow 4 \ell$ decays in order to measure the signal rates of different Higgs boson production modes. For these measurements, the full $2015+2016$ ATLAS data set is used, corresponding to $36.1 \mathrm{fb}^{-1}$ of $p p$ collisions at a centre-of-mass energy of $\sqrt{s}=13 \mathrm{TeV}$. Multivariate techniques are used to isolate the rare vector boson fusion ( $\mathrm{VBF}$ ) production mechanism, allowing for a more precise rate measurement for the process. The measured VBF signal rate is found to be $\mu_{\mathrm{VBF}}=3.95_{-1.48}^{+1.73}$, which is consistent with the Standard Model prediction of one. Limits are set on the presence and strength of $\mathcal{C P}$-odd and non-Standard Model $\mathcal{C P}$-even Higgs boson couplings to gluons and weak bosons. For each coupling hypothesis tested, the observed limits are compatible with the Standard Model, although the best-fit configurations favour the presence of anomalous couplings. 
A new ATLAS electron and photon reconstruction algorithm is presented which uses dynamically-sized clusters to recover radiative energy losses by electrons and positrons within the detector. The resultant "superclusters" improve the expected energy resolution of electrons and photon conversions by up to 40\%. Expected resolution improvements are also found for a number of topologies relevant to the ATLAS physics programme, with $5-8 \%$ improvements in mass resolution found for $Z \rightarrow e^{+} e^{-}$and $J / \psi \rightarrow e^{+} e^{-}$decays, and up to $5 \%$ improvement in the Higgs boson mass resolution in $H \rightarrow 4 \ell$ decays. 


\section{Acknowledgements}

I'd like to thank my supervisor Thomas Koffas, as well as my colleagues Dag Gillberg, Ioannis Nomidis, Christos Anastopoulos, and Jovan Mitrevski for the many years of guidance, advice, and hard work. Your expertise, understanding, and intuition drive me to improve myself to reach the same level, and hopefully pass on those same skills to the next generation of physicists.

Over the course of my graduate studies at Carleton, I grew from a young, impressionable Master's student to a grizzled, mature $\mathrm{PhD}$ candidate, and in that time I experienced a sea change in the makeup of ATLAS graduate students. To the original series: Graham Cree, Jim Lacey, Kate Whalen, Rick Ueno, and Tom McCarthy, thank you for helping me grow as a scientist with your discussions, feedback, and repartee over the years. To the next generation: Stephen Weber, Steven J. Lee, Matthew Park, and Rob Hunter, thanks for keeping me on my toes with discussions of your new research, and the changing ways of the world.

To my parents Antonio and Marcia, and my sisters Lisa and Tanya, thank you for your unwavering love, support, and encouragement. To Jill, thank you for your constant love and friendship, for dealing with all my globetrotting, and for keeping

my spirits up when the work got especially daunting. Each of you has helped shape me as a person, and surely my success is greater for your love and support. 


\section{Table of Contents}

$\begin{array}{ll}\text { Abstract } & \text { ii }\end{array}$

Acknowledgements $\quad$ iv

List of Tables $\quad$ vi

List of Figures vii

List of Abbreviations viii

1 Introduction 1

2 The Standard Model and beyond 4

2.1 Particle content and interactions . . . . . . . . . . . . . 5

2.1.1 Composite particles . . . . . . . . . . . . . 7

2.1.2 Conservation laws . . . . . . . . . . . . . . . 9

2.2 The SM Lagrangian . . . . . . . . . . . . . . . . . . . . 10

2.3 The Higgs mechanism . . . . . . . . . . . . . . . . . 12

2.3.1 Electroweak sector . . . . . . . . . . . . . . . 12

2.3.2 Fermion sector . . . . . . . . . . . . . . . . . . 15

2.3.3 Higgs sector . . . . . . . . . . . . . . 16

2.4 Scattering amplitudes and corrections . . . . . . . . . . . . 17

2.5 Higgs boson production and decay modes . . . . . . . . . . . . . 20

2.6 Beyond Standard Model Higgs physics . . . . . . . . . . . . . . 24

3 The LHC and ATLAS 29

3.1 The Large Hadron Collider . . . . . . . . . . . . . . . . . . . . . . 29

3.1.1 Collision terminology . . . . . . . . . . . . . 31

3.2 The ATLAS detector . . . . . . . . . . . . . . . 34

3.2.1 Inner Detector . . . . . . . . . . . . . . . 36

3.2.2 Calorimeter system . . . . . . . . . . . . . . . . 39 
3.2 .3 Muon Spectrometer . . . . . . . . . . . . . . . . . . . . 44

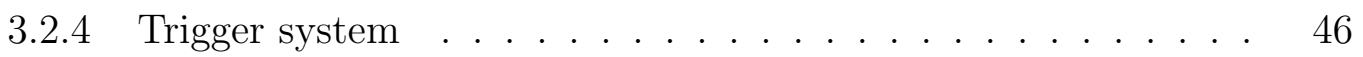

4 Electron, photon reconstruction using superclusters 48

4.1 Overview of electron, photon reconstruction . . . . . . . . . 49

4.1 .1 Clustering . . . . . . . . . . . . . . . . . . . 49

4.1.2 Tracking, loose track-cluster matching, and refitting . . . . . 51

4.1 .3 Electron candidate reconstruction . . . . . . . . . . . . 52

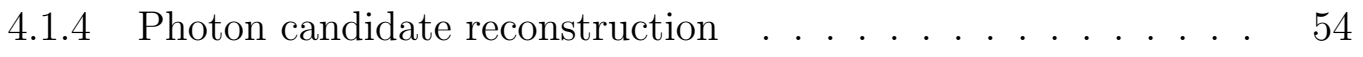

4.1.5 Ambiguity resolution . . . . . . . . . . . . . 55

4.2 Motivation for superclusters . . . . . . . . . . . 56

4.3 Topo-cluster reconstruction $\ldots \ldots \ldots \ldots \ldots$

4.4 Supercluster electron, photon reconstruction . . . . . . . . . 59

4.4 .1 Building EM topoclusters . . . . . . . . . . . . . . . . 59

4.4.2 Defining and building superclusters . . . . . . . . . . 63

4.5 Uncalibrated supercluster performance . . . . . . . . . . . . 68

4.5.1 Electrons, converted photons . . . . . . . . . . . . . 72

4.5 .2 Unconverted photons . . . . . . . . . . . . . . . . 73

4.6 MVA calibration . . . . . . . . . . . . . . . . . . . 73

4.7 Calibrated supercluster performance $\ldots \ldots \ldots \ldots \ldots$

4.7.1 Electrons and converted photons . . . . . . . . . . . 80

4.7 .2 Unconverted photons . . . . . . . . . . . . . . . . 80

4.8 Supercluster performance with simulated pile-up interactions . . . . 80

4.9 Supercluster performance in relevant physics topologies . . . . . . . 84

$4.9 .1 \mathrm{~J} / \psi \rightarrow e^{+} e^{-}$and $Z \rightarrow e^{+} e^{-} \ldots \ldots \ldots \ldots \ldots$

$4.9 .2 H \rightarrow Z Z^{*} \rightarrow 4 \ell \ldots \ldots \ldots \ldots \ldots$

5 Modelling Higgs boson production and decays $\quad 91$

5.1 Gluon fusion Higgs production . . . . . . . . . . . . . . . 93

5.1.1 NNLO production via the POWHEG NNLOPS process . . . . 94

5.1.2 NLO merging via the FxFx process in aMC@NLO . . . . . . 95

5.2 Vector boson fusion, associated production . . . . . . . . . 97

5.3 The Higgs Characterization model . . . . . . . . . . . . . . . . . . 99 
5.3.1 Higgs total width modifications . . . . . . . . . . . 107

5.4 Histogram morphing . . . . . . . . . . . . . . . . 108

6 Datasets, physics object reconstruction, and event selection $\quad 117$

6.1 Dataset and simulated signal samples . . . . . . . . . . 118

6.1.1 SM Higgs boson signal modelling . . . . . . . . . . . . . 119

6.1.2 Higgs boson signal with anomalous couplings . . . . . . . . . 120

6.2 Object reconstruction and selection . . . . . . . . . . 120

6.2 .1 Electron and muon reconstruction . . . . . . . . . . 120

6.2.2 Photon and jet reconstruction and selection . . . . . . . 122

6.3 Event selection . . . . . . . . . . . . . . . . . . . . . . 124

6.3 .1 Pre-selection . . . . . . . . . . . . . . . . . . . . . . . 124

6.3.2 Event-level selection . . . . . . . . . . . . . . . 125

6.4 Four lepton candidate selection . . . . . . . . . . . . . 127

6.5 Analysis categories . . . . . . . . . . . . . . . 130

6.6 Background estimation and modelling . . . . . . . . . . . 133

6.6.1 Reducible background estimation . . . . . . . . . . . 134

6.7 Best prediction scaling for signal with anomalous couplings . . . . . 136

7 The VBF $H \rightarrow Z Z^{*} \rightarrow 4 \ell$ multivariate analysis in ATLAS 138

7.1 Boosted decision trees . . . . . . . . . . . . . . . . 139

7.2 Input sample selection . . . . . . . . . . . . . . . 140

7.3 Input variable selection . . . . . . . . . . . . . . . . . 142

7.3.1 Optimization of input variables . . . . . . . . . . 147

7.4 BDTs and theory uncertainties in $H+2 j$ events . . . . . . . . 151

7.5 Flattening the distribution of $p_{\mathrm{T}}^{H j j} \ldots \ldots \ldots \ldots \ldots \ldots \ldots$

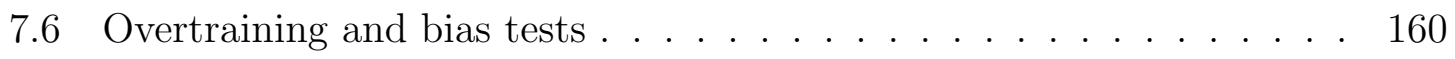

7.7 Discriminants for other categories . . . . . . . . . . . 161

8 Systematic uncertainties $\quad 165$

8.1 Experimental uncertainties . . . . . . . . . . . . . 166

8.2 Theoretical uncertainties . . . . . . . . . . . . . . 168

8.2.1 QCD scale variation, bin migration uncertainties . . . . . 168 
8.2.2 PDF uncertainties . . . . . . . . . . . . . . . 174

8.2.3 Parton showering, underlying event . . . . . . . . . 176

8.2.4 $k$-factor variations with anomalous couplings . . . . . . 178

9 Signal strength measurements, limits on anomalous couplings, and outlook

9.1 Event yields and discriminant shapes . . . . . . . . . . . . . . . 182

9.2 Higgs boson signal strength measurements . . . . . . . . . . . . 186

9.3 Limits on anomalous Higgs boson couplings . . . . . . . . . . . . 189

9.4 Outlook for anomalous Higgs boson coupling limits . . . . . . . . . 193

$\begin{array}{ll}10 \text { Summary } & 205\end{array}$

A Personal contributions to the ATLAS collaboration 208

B Higgs, jet kinematic definitions 212

C Merging scale choice and jet kinematics for ggF FxFx samples 214

D VBF $H \rightarrow 4 \ell$ prospects at HL-LHC $\quad 217$

D.1 The HL-LHC and Phase-II upgrades . . . . . . . . . . . . . . 219

D.2 Higgs boson signal, background simulation . . . . . . . . . . . . 221

D.2.1 Higgs boson signal samples . . . . . . . . . . . . . . . . 222

D.2.2 Background samples . . . . . . . . . . . . . . . . . . . . 223

D.2.3 Truth object smearing and pile-up simulation . . . . . . . . 224

D.2.4 BDT training and variable shapes . . . . . . . . . . 226

D.3 Analysis methodology . . . . . . . . . . . . . 226

D.3.1 Event selection . . . . . . . . . . . . . . . . . . . . . 229

D.3.2 Definition of the VBF signal region . . . . . . . . . . 230

D.3.3 $p_{\mathrm{T}}^{H j j}$ and S-T uncertainties for BDT categories . . . . . . 231

D.4 Statistical analysis and results . . . . . . . . . . . . . 232

$\begin{array}{lll}\text { E } & \text { PYTHIA8 settings } & 236\end{array}$

$\begin{array}{ll}\text { References } & 238\end{array}$ 


\section{List of Tables}

4.1 Cluster size in $n_{\eta} \times n_{\phi}$ cells for different particle types, in the barrel and end-cap regions of the ECAL. . . . . . . . . . . . .

5.1 Partonic cross sections computed at different QCD orders for inclusive, gluon fusion-mediated Higgs production with $m_{H}=125 \mathrm{GeV}$ and $\sqrt{s}=$ $13 \mathrm{TeV}$. The LO and NLO cross section estimates were computed using MAdGrAPH5_aMC@NLO. . . . . . . . . . . . . . . . . . .

6.1 List of input samples for the combined signal model of vector fusion production and associated production, used to build a morphing basis allowing the simultaneous introduction of $\kappa_{H V V}$ and $\kappa_{A V V}$ in the $H V V$ interaction vertex. . . . . . . . . . . . . . . . 121

6.2 List of input samples for the gluon fusion signal model, used to build a morphing basis which allows the introduction of $\kappa_{H V V}$ and $\kappa_{A V V}$ in the $Z Z^{*}$ decay vertex. . . . . . . . . . . . . . . . .

6.3 List of input samples for the gluon fusion signal model describing $\mathcal{C} \mathcal{P}$ mixing via the introduction of $\kappa_{A g g}$ in the ggF production vertex. . . 122

6.4 Trigger list for the $H \rightarrow 4 \ell$ event selection, where rows have been grouped according to trigger type. . . . . . . . . . . . . . 126

6.5 Event selection requirements defining the fiducial phase space of the $4 \ell$ analysis [52]. . . . . . . . . . . . . . . . . . . . . . 128

6.6 List of per-category "best prediction" scale factors for Higgs signal samples with anomalous couplings.

7.1 Table of the shape separation $\left\langle S^{2}\right\rangle$ and BDT importance for a number of kinematic variables with power to separate VBF and ggF Higgs production. Variables are ranked in order of descending $\left\langle S^{2}\right\rangle$, with the corresponding rankings for BDT importance shown. . . . . . . . . . .

7.2 Optimal training variable configurations for each choice of $k$ variables from the full set of $n=11$ possible variables. . . . . . . . . . . . . . . 149 
7.3 Relative uncertainties for the 2-jet enriched region, $\Delta \sigma_{2} / \sigma_{2}$, in bins of rectangular cuts on $p_{\mathrm{T}}^{H j j}$, computed using the Stewart-Tackmann method. . . . . . . . . . . . . . . . . .

9.1 The observed and expected signal and background yields in each reconstructed event category, within the invariant mass range $118<$ $m_{4 \ell}<129 \mathrm{GeV}$, for an integrated luminosity of $36.1 \mathrm{fb}^{-1}$ recorded at $\sqrt{s}=13 \mathrm{TeV}$. The expected yields assume the SM Higgs boson signal with a mass $m_{H}=125.09 \mathrm{GeV}$. Statistical and systematic uncertainties are added in quadrature. . . . . . . . . . . . . . . . . 183

9.2 Expected and observed signal strength values at $36.1 \mathrm{fb}^{-1}$ of $\sqrt{s}=$ $13 \mathrm{TeV}$ data in the $H \rightarrow Z Z^{*} \rightarrow 4 \ell$ channel, made using the inclusive sample, and for each considered Higgs production mode. The relative uncertainties on each measurement are presented in components as $($ statistical $)+($ experimental $)+($ theoretical $) . \ldots \ldots \ldots \ldots$

9.3 Expected and observed 95\% CL for the $\kappa_{A g g}, \kappa_{H V V}$ and $\kappa_{A V V}$ coupling strengths, the best-fit values of each coupling strength, and the corresponding compatibility with the SM expectation, obtained using negative log-likelihood scans performed with $36.1 \mathrm{fb}^{-1}$ of data at $\sqrt{s}=13 \mathrm{TeV}$. The coupling strength $\kappa_{H g g}$ is fixed to the SM value of one in the fit, while $\kappa_{\mathrm{SM}}$ is either fixed to the SM value of one, or left as a free parameter of the fit. . . . . . . . . . . . . . . 191

9.4 The best-fit coupling values and corresponding deviation from the SM expectation, obtained using two-dimensional log-likelihood scans over $\kappa_{H V V}$ and $\kappa_{A V V}$, performed with $36.1 \mathrm{fb}^{-1}$ of data at $\sqrt{s}=13 \mathrm{TeV}$.

D.1 Kinematic selection used to isolate truth-level $H \rightarrow 4 l+2 j$ events. . 229

D.2 Expected event counts before and after inclusive and dijet event selection at $\mathcal{I}=3000 \mathrm{fb}^{-1}$ and $\sqrt{s}=14 \mathrm{TeV}$, assuming a Higgs-boson mass of $m_{H}=125.5 \mathrm{GeV} \ldots \ldots \ldots \ldots \ldots \ldots \ldots \ldots$

D.3 Relative S-T uncertainties $\Delta \sigma_{2} / \sigma_{2}$ in bins of $10 \mathrm{GeV}$ rectangular cuts on $p_{\mathrm{T}}^{H j j}$, computed at $\sqrt{s}=14 \mathrm{TeV}$ using the Stewart-Tackmann method.231 
D.4 Expected signal and background event counts for different jet tracking coverage scenarios at $\mathcal{I}=3000 \mathrm{fb}^{-1}$ and $\langle\mu\rangle=200$ in the region (BDT score) $>0$ and $120<m_{4 l}<130 \mathrm{GeV}$, along with the VBF signal significance and signal strength uncertainty for each scenario. Scenarios are shown where only the statistical uncertainty (top), and also Stewart-Tackmann uncertainties (bottom) are considered in the fit. In the first case, the bracketed terms represent the number of events in the signal region with two selected jets from the primary vertex. . . . . . . . . . . . . . . . . . 235

D.5 Expected signal and background event counts for different jet tracking coverage scenarios at $\mathcal{I}=3000 \mathrm{fb}^{-1}$ and $\langle\mu\rangle=140$ in the region (BDT score) $>0$ and $120<m_{4 l}<130 \mathrm{GeV}$, along with the VBF signal significance and signal strength uncertainty for each scenario. Scenarios are shown where only the statistical uncertainty (top), and also Stewart-Tackmann uncertainties (bottom) are considered in the fit. In the first case, the bracketed terms represent the number of events in the signal region with two selected jets from the primary vertex. . . . . . . . . . . . . . . . . . . 235

E.1 Shower variation settings for the AZNLO tune in Pythia8. . . . . 237 


\section{List of Figures}

2.1 The potential $V(\Phi)$ introduced to the electroweak Lagrangian by the Higgs mechanism, under the condition $\mu^{2}<0$ [23]. The rolling ball represents the development of a vacuum expectation value (VEV) by the neutral component of the scalar doublet $\Phi$, induced by the introduction of a new minimum energy state by the potential. . . . . . . 13

2.2 The fundamental components of Feynman diagrams, particle propagators and interaction vertices. . . . . . . . . . . . 18

2.3 Feynman diagrams for a tree-level, photon-mediated $e^{+} e^{-} \rightarrow e^{+} e^{-}$ process. . . . . . . . . . . . . . . . . . . 19

2.4 Possible corrections to the tree-level $e^{+} e^{-} \rightarrow e^{+} e^{-}$scattering process. $\quad 20$

2.5 Tree-level Feynman diagrams of the four main Higgs boson production mechanisms at the LHC. . . . . . . . . . . . . . . . 21

2.6 Examples of NLO QCD corrections to the ggF Higgs boson production process. For simplicity, the $t \bar{t}$ loop has been enclosed in a filled circle.

2.7 Examples of corrections to the vertices involved in NLO QCD VBF and VH Higgs boson production calculations [22]. . . . . . . . 22

2.8 Tree-level (leading order) Feynman diagrams for notable light-to-medium Higgs boson decay channels searched for at the LHC. . . . . . . . . . 24

2.9 Predicted cross sections of various Higgs boson production methods at $\sqrt{s}=13 \mathrm{TeV}$ as a function of $m_{H}$, the Higgs boson mass [41]. . . .

2.10 Predicted branching ratios of the various Higgs boson decay modes as a function of $m_{H}$, the Higgs boson mass [41] . . . . . . . . 25

2.11 Corrections to the Higgs mass calculation which induce the hierarchy problem. . . . . . . . . . . . . . . . .

3.1 Diagram of the injection chain at the LHC for proton beams. The collider is designed to accelerate each proton beam up to energies of $7 \mathrm{TeV}$. The relative locations of the four main experiments are also labelled. Figure based on Ref. [54]. . . . . . . . . . . . . . . 
3.2 Illustration of the way a QCD Monte Carlo generator would simulate a proton-proton collision in which a hard $2 \rightarrow 2$ scattering process occurs, leading to final state partons with some momentum $p_{\mathrm{T}}$ transverse to the initial proton momenta (hard). Figure based on Ref. [60]. . . .

3.3 Cut-away view of the ATLAS detector. The detector stands $25 \mathrm{~m}$ high and is $44 \mathrm{~m}$ long, with a total weight of approximately 7000 tonnes [55]. 34

3.4 (Left) Layout of the ATLAS detector in Cartesian coordinates, with definitions of $\theta$ and $\phi$, relative to their rotational axes. (Right) Pseudorapidity and its relation to the $z$-axis. . . . . . . . . . .

3.5 Sensors and structural elements of the Inner Detector, along with the trajectory of a charged track with $p_{\mathrm{T}}=10 \mathrm{GeV}$ in the barrel region $(\eta=0.3)[62] \ldots \ldots \ldots \ldots \ldots$. . . . . . . . . . . . . . . 37

3.6 Cutaway view of the layers of the ATLAS calorimeter system [65]. . . 39

3.7 Sketch of the segmentation of a barrel module in the ECAL, showing the different layers and ganging of electrodes in the $\phi$-direction. The cell granularity in $\Delta \eta \times \Delta \phi$ is shown for each layer, along with the trigger towers [55]. . . . . . . . . . . . . . . . . 41

3.8 Cutaway view of the of the ATLAS Muon Spectrometer subsystem [55]. 44

4.1 Diagram of an example supercluster showing a seed electron cluster and a satellite photon cluster. . . . . . . . . . . . 57

4.2 Algorithm flow diagram for the new electron and photon reconstruction. 60

4.3 Distributions of $f_{\mathrm{EM}}$ and reconstruction efficiency for true electron clusters (black) and pile-up (red). . . . . . . . . . . . . .

4.4 Distance in $\Delta \eta$ and $\Delta \phi$ between the barycentres of seed and satellite clusters in simulated single electron clusters. . . . . . . . . . . . . 64

4.5 Steps of the superclustering algorithm for electrons and photons. . . 65

4.6 Distances in $\Delta \eta$ and $\Delta \phi$ between cluster barycentres under two scenarios: Between simulated primary electron topo-clusters and true secondary topo-clusters (black); and between simulated primary electron topo-clusters, and non-primary EM topo-clusters which are matched to the primary track (red). A window of $\Delta \eta \times \Delta \phi=0.125 \times 0.3$ is also demarcated on each plot. . . . . . . . . . . . . . 
4.7 Effect of restricting cluster size in $|\eta|$ on a sample electron supercluster, in the second EM calorimeter layer. The $z$-axis scale is the base- 10 logarithm of the cell energy. . . . . . . . . . . . . . . .

4.8 Average shower containment distributions for electrons reconstructed via sliding window and supercluster-based approaches. Included also are superclusters built using topo-clusters allowed unrestricted growth

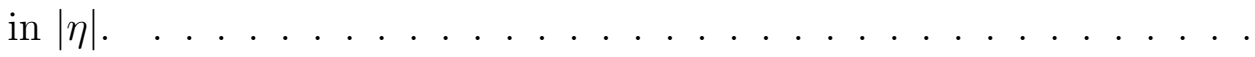

4.9 Distributions of the average number of cells contained in electron clusters reconstructed via sliding window and supercluster-based approaches. Included also are superclusters built using topo-clusters allowed unrestricted growth in $|\eta| \ldots \ldots \ldots \ldots$. . . . . . . . . . . 70

4.10 Diagram showing the IQR of a unit-width Normal distribution, where $Q_{1}$ and $Q_{3}$ are the first and third quartiles of the distribution. . . . 71

4.11 An example of the change in uncalibrated energy response for electrons in different truth energy regions, built using sliding window clusters. .

4.12 Comparison of uncalibrated IQE values between sliding window (red) and supercluster (blue) electrons, using samples of single electron events. 74

4.13 Comparison of uncalibrated Gaussian peak values between sliding window (red) and supercluster (blue) electrons, using samples of single electron events. . . . . . . . . . . . . . . . . . . 74

4.14 Comparison of uncalibrated IQE values between sliding window (red) and supercluster (blue) converted photons, using samples of single converted photon events. . . . . . . . . . . . . . . 75

4.15 Comparison of uncalibrated Gaussian peak values for sliding window (red) and supercluster (blue) converted photons, using samples of single converted photon events. . . . . . . . . . . . . .

4.16 Comparison of uncalibrated IQE values between sliding window (red) and supercluster (blue) unconverted photons, using samples of single unconverted photon events. . . . . . . . . . . . . 76

4.17 Comparison of uncalibrated Gaussian peak values for sliding window (red) and supercluster (blue) unconverted photons, using samples of single unconverted photon events. . . . . . . . . . . . . . 
4.18 Distributions of cluster-based MVA calibration training variables for sliding window and supercluster-based electrons and photons. . . . . 79

4.19 Distributions of track-based MVA calibration training variables for sliding window and supercluster-based converted photons using the new reconstruction software. . . . . . . . . . . . . . .

4.20 Comparison of calibrated IQE values between sliding window (red) and supercluster (blue) electrons, using samples of single electron events. .

4.21 Comparison of calibrated IQE values between sliding window (red) and supercluster (blue) converted photons, using samples of single converted photon events. . . . . . . . . . . . . . .

4.22 Comparison of calibrated IQE values between sliding window (red) and supercluster (blue) unconverted photons, using samples of single unconverted photon events. . . . . . . . . . . . .

4.23 Calibrated energy response resolution, expressed in terms of IQE, for simulated single electrons at different $\langle\mu\rangle$ levels. Sliding window-based response is shown in red, while the supercluster-based response is shown in blue. . . . . . . . . . . . . . . . .

4.24 Calibrated energy response resolution, expressed in terms of IQE, for simulated single converted photons at different $\langle\mu\rangle$ levels. Sliding window-based response is shown in red, while the supercluster-based response is shown in blue. . . . . . . . . . . . . . . .

4.25 Calibrated energy response resolution, expressed in terms of IQE, for simulated single unconverted photons at different $\langle\mu\rangle$ levels. Sliding window-based response is shown in red, while the supercluster-based response is shown in blue. . . . . . . . . . . . . . . .

4.26 Invariant mass distribution of the dielectron system from simulations of reconstructed $J / \psi \rightarrow e^{+} e^{-}$decays (left) and $Z \rightarrow e^{+} e^{-}$decays (right). . . . . . . . . . . . . . . . . .

4.27 Superimposed distributions of the invariant mass of simulated reconstructed four lepton candidates using $H \rightarrow Z Z^{*} \rightarrow 4 \ell$ analysis selection. The resolution values are derived from the width of a fitted Double Crystal Ball function. . . . . . . . . . . . . . . . 
5.1 Comparison of the inclusive Higgs $p_{\mathrm{T}}$ spectrum of various generators: HRes, Powheg NnLops, and MadGraph5_aMC@NLO FxFx, with each weighted to the best available $\left(\mathrm{N}^{3} \mathrm{LO}\right)$ cross section prediction. .

5.2 Kinematic shapes of important variables in $\geq 2$-jet ggF final states for different MC generators, with each distribution scaled to unit area. .

5.3 Comparison of the LO and NLO QCD differential cross section distributions for VBF Higgs boson production at $\sqrt{s}=13 \mathrm{TeV}$. The inclusive cross section of the NLO QCD component has been reweighted to match the best-known NNLO QCD cross section. . . . . . . . . . . . 100

5.4 Comparison of the LO and NLO QCD differential cross section distributions for hadronic VH Higgs boson production at $\sqrt{s}=13 \mathrm{TeV}$. The inclusive cross section of the NLO QCD component has been reweighted to match the best-known NNLO QCD cross section. . . . 101

5.5 Comparison of the kinematics in LO VBF + VH Higgs production under different coupling scenarios using the Higgs Characterization model.105

5.6 Comparison of the kinematics in NLO-merged ggF Higgs production for $\mathcal{C P}$-even and $\mathcal{C P}$-odd coupling scenarios using the Higgs Characterization model. . . . . . . . . . . . . . . 106

5.7 Changes to the Higgs production cross sections (left) and $Z Z^{*}$ branching ratio (right) as a function of different anomalous coupling strength constants in the Higgs Characterization model. . . . . . . . . . . . . . 107

5.8 The inverse of the change in the Higgs boson total width $\Gamma_{H}$ as a function of different anomalous couplings in the Higgs Characterization model, which serves as a correction factor to the branching ratios predicted by the HC model. . . . . . . . . . . . . . . . . . . . . . 108

5.9 Comparison of $n_{\text {eff }} / n_{\text {tot }}$ for morphing bases composed of $\left(\kappa_{H g g}, \kappa_{A g g}\right)=$ $(1,0),(0,1)$, and $(1,-1)$ (left), and $\kappa_{A g g}$ in $[-0.5,0,0.5]$ with $\kappa_{H g g}=1$

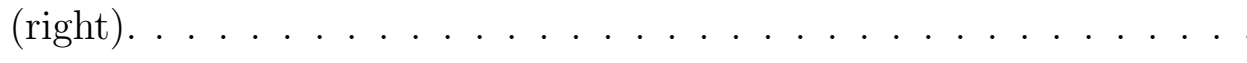

5.10 Comparison of $n_{\text {eff }} / n_{\text {tot }}$ in two dimensions $\left(\kappa_{H g g}\right.$ and $\left.\kappa_{A g g}\right)$ for morphing bases used to interpolate between values of $\kappa_{H g g}$ and $\kappa_{A g g}$. The SM equivalent scenario of $\kappa_{H g g}=1$ is marked with a dotted line. . . . . . 114 
5.11 Distributions of $n_{\mathrm{eff}} / n_{\text {tot }}$ for morphing bases built to interpolate beween values of $\kappa_{A V V}$ and $\kappa_{H V V}$ in $\mathrm{VBF}+\mathrm{VH}$ Higgs boson production, and $\kappa_{A g g}$ in $\operatorname{ggF}$ production.

6.1 Definitions of the impact parameters $d_{0}, z_{0}$, and $z_{0} \sin \theta$ for a particle track crossing the $z$-axis / beam line. . . . . . . . . . . . . . . . 127

6.2 Flow chart of event categorization in the $4 \ell$ analysis for $\sqrt{s}=13 \mathrm{TeV} .132$

7.1 Schematic diagram of a binary decision tree. . . . . . . . . . . . . 140

7.2 Comparison of truth- and reconstruction-level kinematics for ggF $H+$ $2 j$ events. The truth-level events are generated at NLO QCD using the Powheg HJJ generator, while the reconstruction-level events are generated at LO using the Powheg NNLOPs generator. . . . . . . .

7.3 Comparison of truth- and reconstruction-level kinematics for VBF $H+$ $2 j$ events. Both truth- and reconstruction-level events are generated using the Powheg VBFH generator. . . . . . . . . . . . . .

7.4 Discriminating variables considered for use in training the BDT to separate $\mathrm{VBF}$ and $\mathrm{ggF}$ production in the $H \rightarrow Z Z^{*} \rightarrow 4 \ell$ channel. . 146

7.5 Linear correlation coefficients for the optimized list of training variables, produced using truth-level samples of $\mathrm{VBF}$ and ggF $H \rightarrow 4 \ell$ events, generated at $\sqrt{s}=14 \mathrm{TeV}$ using the Powheg generator. . . . 150

7.6 Effect of cuts on $p_{\mathrm{T}}^{H j j}$ on the parton-level ggF $H+2 j$ differential cross section $d \sigma / d p_{\mathrm{T}}$, along with the scale variation error bands defined by $\mu_{\mathrm{R}}=\mu_{\mathrm{F}}=m_{H} / 2$ and $2 m_{H}$. All distributions are derived using MCFM with $m_{H}=125 \mathrm{GeV}$, requiring jet $p_{\mathrm{T}}$ above $30 \mathrm{GeV}$, and $m_{\mathrm{jj}}>120 \mathrm{GeV} .152$

7.7 Effect of cuts on $p_{\mathrm{T}}^{H j j}$ on the parton-level ggF $H+2 j$ differential cross section $d \sigma / d p_{\mathrm{T}}$, along with the scale variation error bands defined by $\mu_{\mathrm{R}}=\mu_{\mathrm{F}}=m_{H} / 2$ and $2 m_{H}$. Also shown are the symmetrized error bands computed using the Stewart-Tackmann method. All distributions are derived using MCFM with $m_{H}=125 \mathrm{GeV}$, requiring jet $p_{\mathrm{T}}$ above $30 \mathrm{GeV}$, and $m_{\mathrm{jj}}>120 \mathrm{GeV}$. . . . . . . . . . . . . . . . 154 
7.8 A comparison of the shape of $p_{\mathrm{T}}^{H j j}$ in $\mathrm{ggF} H+2 j$ events, before and after "flattening" the distribution to create $p_{\mathrm{T}}^{{ }^{H j j}}$, for a threshold value of $30 \mathrm{GeV}$. . . . . . . . . . . . . . . . . . .

7.9 Distribution of expected VBF significance (left) and signal strength uncertainty (right) using a likelihood fit across 10 BDT bins in $[-1,1]$, under different flattening thresholds of $p_{\mathrm{T}}^{H j j}$. Also shown are analogous values for BDTs trained using the Run-I variables (blue line), and a BDT trained without $p_{\mathrm{T}}^{H j j}$ (red line) . . . . . . . . . . . . 158

7.10 Distribution of $p_{\mathrm{T}}^{H j j}$ vs. BDT score in $\operatorname{ggF} H \rightarrow Z Z^{*} \rightarrow 4 \ell$ events with $m_{\mathrm{jj}}>120 \mathrm{GeV}$ and $p_{\mathrm{T}}^{H j j}<50 \mathrm{GeV}$, indicating that the trained BDT does not have a visible correlation with $p_{\mathrm{T}}^{H j j}$ in this region of phase space. . . . . . . . . . . . . . . . . .

7.11 Superimposition of the BDT response for the training and testing samples of $\mathrm{VBF}$ and $\mathrm{ggF} H \rightarrow 4 \ell$ events. . . . . . . . . . . . . . . 160

7.12 Shape of $m_{4 \ell}$ in different regions of the BDT discriminant for both VBF (left) and ggF (right) production. . . . . . . . . . . . . 161

7.13 Linear correlation coefficients for the BDT training produced using fully-simulated samples of VBF and ggF $H \rightarrow 4 \ell$ events. . . . . . . . 162

7.14 Per-category discriminants used in the $H \rightarrow Z Z^{*} \rightarrow 4 \ell$ analysis, shown for each major considered Higgs boson production mode. . . .

8.1 Theoretical uncertainties on the ggF cross section in each $4 \ell$ analysis bin, computed at $\sqrt{s}=13 \mathrm{TeV}$, due to QCD scale variations, bin migrations, and quark mass effects. . . . . . . . . . . . .

8.2 Theoretical uncertainties on the shapes of the BDT discriminants for $\operatorname{ggF} H+2 j$ events due to QCD scale variations, bin migrations, and quark mass effects.

8.3 Theoretical uncertainties due to PDF eigenvector and $\alpha_{s}$ variations on the ggF cross section in each $4 \ell$ analysis bin, computed at $\sqrt{s}=13 \mathrm{TeV}$, using PDF4LHC as the nominal PDF choice. . . . . . . . . . . . . 176

8.4 Uncertainties due to variations in the choice and configuration of the parton shower used to generate truth-level ggF events. . . . . . . . . 178 
8.5 Shape uncertainties due to choice of SMC generator and tune for the BDT discriminants in $\mathrm{ggF} H \rightarrow Z Z^{*} \rightarrow 4 \ell$ events. . . . . . . . . . . 179

8.6 Shape uncertainties due to variations in the nominal PYTHIA8 AZNLO tune for the BDT discriminants in ggF $H \rightarrow Z Z^{*} \rightarrow 4 \ell$ events. . . . 180

9.1 Inclusive distribution of the invariant mass $m_{4 \ell}$ in data, superimposed on MC normalized to the SM prediction for $36.1 \mathrm{fb}^{-1}$ at $\sqrt{s}=13 \mathrm{TeV}$.

9.2 Distributions of the per-category discriminants in data, superimposed on MC normalized to the SM prediction for $\mathcal{L}=36.1 \mathrm{fb}^{-1}$ recorded at $\sqrt{s}=13 \mathrm{TeV}$.

9.3 Distributions of the kinematic variables used to train the VBF discriminant in both data and $\mathrm{MC}$, shown in the kinematic region with $m_{\mathrm{jj}}>120 \mathrm{GeV}$ and $p_{\mathrm{T}}^{j_{1}}<200 \mathrm{GeV} \ldots \ldots \ldots \ldots$. . . . . . . .

9.4 The observed inclusive signal strength measurement (top), as well as the signal strength per production mode (bottom), for $36.1 \mathrm{fb}^{-1}$ of data recorded at $\sqrt{s}=13 \mathrm{TeV} \ldots \ldots \ldots \ldots$

9.5 Observed (solid black line) and SM expected (dashed blue line) negative log-likelihood scans for (a) $\kappa_{A g g}$, (b) $\kappa_{H V V}$ and (c) $\kappa_{A V V}$ coupling strengths using $36.1 \mathrm{fb}^{-1}$ of data at $\sqrt{s}=13 \mathrm{TeV}$. The horizontal lines indicate the value of the test statistic corresponding to the $68 \%$ and 95\% CL intervals for the parameter of interest. . . . . . . . . . 190

9.6 Observed (black) and SM expected (blue) contours of the 2D negative log-likelihood at 95\% CL for the $\kappa_{H V V}$ and $\kappa_{A V V}$ coupling strengths with $36.1 \mathrm{fb}^{-1}$ of data at $\sqrt{s}=13 \mathrm{TeV}$. The coupling strength $\kappa_{H g g}$ is fixed to the SM value of one in the fit. The coupling strength $\kappa_{\mathrm{SM}}$ is (a) fixed to the SM value of one or (b) left as a free parameter of the fit. . . . . . . . . . . . . . . . .

9.7 Unfolded differential cross sections of $p_{\mathrm{T}}^{H}$ in bins of $N_{j}=0,1$, and $\geq 2$ jets, made using $36.1 \mathrm{fb}^{-1}$ of $H \rightarrow Z Z^{*} \rightarrow 4 \ell$ data recorded at $\sqrt{s}=13 \mathrm{TeV}[52] \ldots \ldots \ldots \ldots \ldots \ldots$. . . . . . . . . . . . . . . . . . . . .

9.8 Comparison of $Z Z^{*} \mathrm{SM}$ differential cross section predictions of $p_{\mathrm{T}}^{H}$ vs. $N_{j}$ at $\sqrt{s}=13 \mathrm{TeV}$ with a number of BSM hypotheses, derived using the Higgs Characterization model. . . . . . . . . . . . . . . . . . . . . 198 
9.9 Example of $\Delta \chi^{2}$ test statistic distributions for null and alternate hypotheses, along with the definition of the $p$-value $p_{\text {alt }} \ldots \ldots \ldots 200$

9.10 Example of the method used to generate toys (pseudo-experiments) from expected differential cross sections, which are later used to generate $\Delta \chi^{2}$ test statistic distributions. . . . . . . . . . . . . . . . 201

9.11 Distributions of $\Delta \chi^{2}=\chi_{\mathrm{BSM}}^{2}-\chi_{\mathrm{SM}}^{2}$ for various hypothesis tests, computed using toys centred around the SM hypothesis, as well as toys centred around different BSM hypotheses. . . . . . . . . . . . 202

9.12 Expected exclusion limits on anomalous couplings in the Higgs Characterization model, set using differential cross section pseudo-data generated around the SM at $\sqrt{s}=13 \mathrm{TeV}$. Shown are simultaneous constraints on BSM $\mathcal{C P}$-even and odd coupling strengths $\kappa_{H V V}$ and $\kappa_{A V V}$ (left), and constraints on $\mathcal{C P}$-odd coupling strengths $\kappa_{A g g}$ and $\kappa_{A V V}$

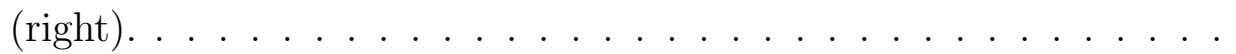

C.0 Comparison of relevant Higgs boson and jet kinematic quantities for samples of NLO-merged ggF events generated using the FxFx merging process in MADGRAPH5_aMC@NLO. Results are shown for choices of $\mu_{q}=30,40$, and $50 \mathrm{GeV} \ldots \ldots \ldots \ldots \ldots$

D.1 Comparison of the BDT training variable shapes for VBF $H \rightarrow 4 \ell$ events generated at $\sqrt{s}=14 \mathrm{TeV}$. The events are shown for each upgrade scenario, as well as for the case of no simulated pile-up. . .

D.2 Comparison of the BDT training variable shapes for $\mathrm{ggF} H \rightarrow 4 \ell$ events generated at $\sqrt{s}=14 \mathrm{TeV}$. The events are shown for each upgrade scenario, as well as for the case of no simulated pile-up. . .

D.3 BDT classifier distributions for Low, Middle, and Reference detector layouts at $\langle\mu\rangle=200$ and pile-up efficiency of 0.02 , as well as the response for a BDT trained exclusively on hard-scatter jets. . . . . . 233

D.4 BDT classifier distributions for Low, Middle, and Reference detector layouts at $\langle\mu\rangle=140$ and pile-up efficiency of 0.02 , as well as the response for a BDT trained exclusively on hard-scatter jets. . . . . . 234 


\section{List of Abbreviations}

\begin{tabular}{ll} 
ATLAS & A Toroidal LHC Apparatus \\
BLPTW & Boughezal-Liu-Petriello-Tackmann-Walsh \\
BSM & Beyond Standard Model \\
CL & Confidence Levels \\
CRs & Control Regions \\
CSC & Cathode-Strip Chambers \\
DM & Dark Matter \\
ECAL & Electromagnetic Calorimeter \\
EFT & Effective Field Theory \\
EMEC & Electromagnetic End-cap Calorimeter \\
EW & Electroweak \\
FCal & Forward Calorimeter \\
FSR & Final State Radiation \\
GgF & Gluon Fusion \\
GRL & Good Runs List \\
GSF & Gaussian Sum Filter \\
HC & Higgs Characterization \\
HCAL & Hadronic Calorimeter \\
HEC & Hadronic End-cap Calorimeter \\
\hline
\end{tabular}




\begin{tabular}{|c|c|}
\hline HL-LHC & High-Luminosity LHC \\
\hline HLT & High Level Trigger \\
\hline HS & Hard Scattering \\
\hline IBL & Insertable B-Layer \\
\hline ID & Inner Detector \\
\hline IQE & Effective Interquartile Range \\
\hline IQR & Interquartile Range \\
\hline ISR & Initial State Radiation \\
\hline JVF & Jet Vertex Fraction \\
\hline JVT & Jet Vertex Tagger \\
\hline LCCs & Linear Correlation Coefficients \\
\hline LHC & Large Hadron Collider \\
\hline LHE & Les Houches Event \\
\hline LL & Leading-logarithm \\
\hline LO & Leading Order \\
\hline MC & Monte Carlo \\
\hline MDT & Monitored Drift Tube \\
\hline MiNLO & Multi-Scale Improved NLO \\
\hline MLE & Maximum Likelihood Estimate \\
\hline MPI & Multiple Parton Interactions \\
\hline MS & Muon Spectrometer \\
\hline
\end{tabular}




\begin{tabular}{|c|c|}
\hline MVA & Multivariate Analysis \\
\hline NLL & Next-to-leading-logarithm \\
\hline NLO & Next-to-leading-order \\
\hline NNLO & Next-to-next-to-leading-order \\
\hline PDF & Parton Distribution Function \\
\hline PSB & Proton Synchrotron Booster \\
\hline PV & Primary Vertex \\
\hline QCD & Quantum Chromodynamics \\
\hline QFT & Quantum Field Theory \\
\hline Rol & Regions of Interest \\
\hline RPC & Resistive Plate Chambers \\
\hline S-T & Stewart-Tackmann \\
\hline SCT & Semiconductor Tracker \\
\hline SFOS & Same-flavour, Opposite-sign \\
\hline SM & Standard Model of Particle Physics \\
\hline SMC & Shower Monte Carlo \\
\hline SPS & Super Proton Synchrotron \\
\hline SR & Signal Region \\
\hline TGC & Thin Gap Chambers \\
\hline TMVA & Toolkit for Multivariate Data Analysis \\
\hline TRT & Transition Radiation Tracker \\
\hline
\end{tabular}




$\begin{array}{ll}\text { ttH } & \text { Top-anti-top-associated Production } \\ \text { UE } & \text { Underlying Event } \\ \text { VBF } & \text { Vector Boson Fusion } \\ \text { VEV } & \text { Vacuum Expectation Value } \\ \text { VH } & \text { Weak Boson-associated Production } \\ \text { WIMPs } & \text { Weakly Interacting Massive Particles }\end{array}$




\section{Chapter 1}

\section{Introduction}

The Standard Model of Particle Physics (SM) is undoubtedly the most extensivelytested - and well-validated - fundamental theory of nature. Prior to 2012, the most prominent unobserved component of the Standard Model was the Higgs boson. The existence of the Higgs boson is a consequence of the Higgs mechanism, which explains how fundamental particles acquire mass. The simultaneous discovery of a Standard Model Higgs boson-like particle by ATLAS and CMS in 2012 [1, 2] validated both the theoretical predictions given by the mechanism, as well as the existence of a Higgs boson-like particle, thereby "completing" the Standard Model.

In spite of the completion of the model, and for all of the experimental validation it has received, there are gaps in our understanding of modern physics that the Standard Model cannot explain. For example, the structure of the SM would seem to suggest a highly massive Higgs boson, but the comparative "lightness" of the observed mass seems to contradict this expectation, necessitating a strict tuning of the model to bring the mass down to the observed level. Likewise, the SM does not contain any particles which can adequately describe the "dark" matter which comprises $27 \%$ of 
the matter in the universe.

In light of these prominent inconsistencies with the Standard Model, we necessarily enter a phase beyond discovery where we must probe and understand the properties of the Higgs boson with more detail. For example, studies have been performed in previous years to set limits on the spin and charge-parity of the Higgs boson $[3,4,5,6]$, which is nominally a neutral, spin- 0 , parity-even particle. The results of theses studies can tell us broadly what new physics has not yet been excluded, and by proxy, which of the many existing Beyond Standard Model (BSM) theories are still viable for study.

The overarching purpose of this thesis is to present new measurements and constraints on Higgs boson properties using ATLAS data recorded in 2015 and 2016 at a centre-of-mass energy of $13 \mathrm{TeV}$. In particular, measurements of the signal strengths for different Higgs boson production modes are presented, along with limits on the presence of anomalous Higgs boson couplings to weak bosons and gluons. The latter approach can tell us what kinds of BSM phenomena are excluded - or, conversely, not yet excluded - by the data. These studies were made in the $H \rightarrow Z Z^{*} \rightarrow 4 \ell$ decay channel, which is well-suited for precision measurements due to its relatively high signal-to-background ratio, and the good experimental precision of its final-state decay products. A new and improved method of reconstructing electrons and photons within ATLAS is also presented, which has become the standard method within the reconstruction software as of late 2017.

Chapter 2 of this text presents an overview of the Standard Model of Particle Physics, describing the nature of the fundamental particles and their interactions. The primary focus of this chapter is the description of the Higgs mechanism and generation of the Higgs boson, as well as its various production and decay modes. 
This chapter also presents a broad overview of some motivating factors for the study of BSM physics, as well as some notable models relevant to Higgs physics at the Large Hadron Collider (LHC).

Chapter 3 presents technical descriptions of the LHC and the ATLAS decector. Chapter 4 presents the new electron and photon reconstruction algorithm used in ATLAS, for which the author was a primary developer. The motivation and technical implementation of the algorithm are described in detail, and validation studies are presented to demonstrate both that the method improves energy and mass resolution for several key physics processes, and is robust against the higher-energy conditions expected from the LHC in the future.

Chapter 5 provides a detailed look at how Higgs boson production and decay are modelled within ATLAS, with a particular emphasis on the $H \rightarrow Z Z^{*} \rightarrow 4 \ell$ decay channel. Also presented is a discussion of effective field theories, and how they are used to introduce anomalous Higgs boson couplings to the SM. A method to interpolate efficiently between BSM hypotheses is also introduced. The author's contributions to modelling gluon fusion-mediated Higgs boson production are detailed, both for SM and BSM scenarios, and in the context of interpolating between BSM hypotheses.

Chapter 6 describes how detector output from ATLAS is used to reconstruct the energy and trajectory of particles, such as electrons, muons, as well as the quantum-chromodynamical interactions of quarks and gluons. The event selection of the $H \rightarrow Z Z^{*} \rightarrow 4 \ell$ analysis is also detailed, showing how candidate $4 \ell$ events are isolated from the billions of collisions recorded by the ATLAS detector.

Chapter 7 describes the derivation and optimization of a multivariate analysisbased approach to search for vector boson fusion-mediated Higgs boson production, 
which is a rare production process, but provides a very distinct experimental signature of a Higgs boson with two widely-separated quarks (which form jets in ATLAS nomenclature). In conjunction with the $H \rightarrow Z Z^{*} \rightarrow 4 \ell$ decay, this production mode serves as an ideal vehicle to search for non-Standard Model physics in the weak interactions of the Higgs boson. The author was the primary developer of this analysis, which was implemented in the context of a broader effort to precisely measure Higgs boson production rates in the $4 \ell$ decay channel. This methodology is further applied in Appendix D to derive prospective results for the visibility of this process at the High-Luminosity LHC.

Chapter 8 describes the sources of systematic uncertainty present in analyses in the $H \rightarrow Z Z^{*} \rightarrow 4 \ell$ channel, as well as the methods by which their effects are estimated. The author's contributions to assessing gluon fusion-related uncertainties are detailed, with a particular emphasis on how the uncertainties on the Higgs boson signal yield predictions are affected by the division of events into exclusive categories based on the number of final-state jets.

Chapter 9 details the statistical analyses in the $H \rightarrow Z Z^{*} \rightarrow 4 \ell$ channel which implement the author's work. The multivariate discriminant of Chapter 7 is incorporated into a larger analysis which measures the rate of each Higgs boson production mode relative to Standard Model expectations. Furthermore, the Higgs boson signal modelling studies of Chapter 5 are incorporated into an analysis to set limits on the presence of anomalous couplings in Higgs boson production and decay. The results of these analyses are presented for the full ATLAS 2015+2016 dataset, which encompasses $36.1 \mathrm{fb}^{-1}$ of data recorded at $\sqrt{s}=13 \mathrm{TeV}$. 


\section{Chapter 2}

\section{The Standard Model and beyond}

Our modern understanding of physics is governed by the Standard Model of Particle Physics, as formulated through quantum field theory (QFT) [7]. The SM describes the properties of the fundamental components of matter, as well as how they interact. Furthermore, embedded within the SM is a description of how the fundamental particles acquire mass, known as the Nambu-Polyakov-Migdal-Anderson-Brout-EnglertHiggs-Guralnik-Hagen-Kibble mechanism [8, 9, 10, 11]. This mathematical framework is frequently referred to as the Higgs mechanism, as Peter Higgs first published that this same mechanism predicted the existence of a new, massive boson that could be observed through its decays - namely, the Higgs boson [12].

This chapter will give an overview of the physics of the Standard Model, and motivate the search for evidence of Beyond Standard Model physics. Section 2.1 details the particle content of the model, with Section 2.2 explaining how these particles interact. Section 2.3 provides an explanation of the Higgs mechanism, including the origins of the Higgs boson. Section 2.4 presents a pedagogical overview of how quantum mechanical scattering processes are calculated, which will be of relevance to later 
chapters. Section 2.5 details the various production and decay modes of the Standard Model Higgs boson. Lastly, Section 2.6 presents a motivation for searching for BSM physics, as well as a broad overview of popular BSM theories.

\subsection{Particle content and interactions}

According to the Standard Model, the entire known universe is composed of two types of particles: bosons and fermions. Standard Model bosons are integer-spin particles which can mediate interactions between fermions, and in some cases, between each other, as expanded upon in Section 2.2. The photon $(\gamma)$ is a spin-1, massless boson, and famously mediates the electromagnetic force between charged particles. The weak bosons, $W^{ \pm}$and $Z$, are massive, spin-1 bosons which mediate the weak nuclear force (or simply the weak force). The gluon $(g)$ is a spin-1 boson, and is theoretically massless ${ }^{1}$. The gluon mediates the strong nuclear force (or strong force), which is exclusive to quarks, and described using the framework of quantum chromodynamics (QCD). All the preceding particles are examples of gauge bosons, which are the force carrier components of any particle physics model. Lastly, the Higgs boson is a massive, spin-0 boson, and while it is not a gauge boson, it can exist as an intermediary particle which decays to a variety of final states, as explained in Section 2.5.

The fermion family contains entirely spin- $1 / 2$ particles, and is composed of leptons and quarks. The lepton group is composed of three generations or flavours - electrons, muons, and tau particles - with each having a corresponding neutrino: $\nu_{e}, \nu_{\mu}$, and $\nu_{\tau}$, respectively. The $e, \mu$, and $\tau$ all have integer charge of -1 , while all neutrinos are neutral, and each has its own corresponding anti-particle, denoted $\bar{\ell}$ or $\bar{\nu}_{\ell}$. Charged

\footnotetext{
${ }^{1}$ Limits have been set on the gluon mass of $m_{g}<1 \mathrm{MeV}$ [13].
} 
leptons and neutrinos most commonly interact through the weak nuclear force, which allows leptons to change flavour. For example, a muon may permute to an electron by radiating a negatively-charged weak boson as,

$$
\mu^{-} \rightarrow W^{-}+\nu_{\mu} \rightarrow \nu_{\mu}+e^{-}+\bar{\nu}_{e}
$$

The mass hierarchy of leptons is arranged as $m_{e}<m_{\mu}<m_{\tau}$, though it has not yet been confirmed if the same holds true for the analogous neutrinos $[14,15]$.

Quarks are similarly found in two types: up-type, composed of the top, up, and charm quarks; and down-type, composed of bottom, down, and strange quarks. The arrangement of quarks into up- and down-type also forms three generations,

$$
\left(\begin{array}{l}
u \\
d
\end{array}\right), \quad\left(\begin{array}{l}
c \\
s
\end{array}\right), \quad\left(\begin{array}{l}
t \\
b
\end{array}\right) .
$$

Like the different lepton flavours, it is possible for an up-type quark to permute to a down-type quark through the weak nuclear force, and vice versa. Generally speaking, quarks will decay weakly to the next massive quark, leading to a pattern of the form,

$$
t \rightarrow b \rightarrow c \rightarrow s \rightarrow u \leftrightarrow d
$$

A notable difference to the leptons is that quarks have fractional charge, with the up-type quarks having charge $+\frac{2}{3}$, and the down-type quarks having charge $-\frac{1}{3}$. The leptons, quarks, and neutrinos all have anti-matter equivalents, with leptons and quarks being Dirac fermions, meaning that their anti-matter equivalents are unique particles. Whether neutrinos are Dirac fermions is not yet known; numerous experiments are ongoing $[16,17,18]$ to determine whether neutrinos are in fact Majorana fermions, meaning they are their own anti-particles. 
As previously mentioned, the interactions of fermions and bosons are described by quantum field theory. Within this framework, predictions can be made using perturbation theory, which treats interactions between particles as small "perturbations" to a system which is otherwise in a state that is mathematically "stable". Interactions between particles can be dealt with in terms of perturbation theory because the relative strengths of each force are quantified in terms of coupling constants which are typically numerically small. For example, the electromagnetic force has a coupling constant of $\mathcal{O}\left(10^{-2}\right)$, while the weak nuclear force has a coupling constant of $\mathcal{O}\left(10^{-7}\right)$ to $\mathcal{O}\left(10^{-6}\right)$. Conversely, the coupling constant of the strong nuclear force is $\mathcal{O}(1)$. This large disparity in strength for the strong nuclear force means that in low energy regimes, or long-range processes, strong interactions cannot be described using perturbation theory. However, for high energy or short-range interactions characteristic of collider physics experiments, the formerly-divergent expression becomes suppressed by powers of the energy scale of the process, allowing for perturbative QCD [19].

\subsubsection{Composite particles}

A notable property of quarks is that it is possible for bound states of two, three, or four (or more) quarks to exist, which are collectively known as hadrons. In particular, two-quark hadrons are known as mesons, while three-quark hadrons are known as baryons. The key to the existence of hadrons is the colour charge, which is specific to the strong nuclear force. Quarks come in three colours - red, green, and blue with anti-particles carrying anti-colour (anti-red, anti-green, anti-blue). Analogous to electric charge, the sum of the three colours red + green + blue produces a "colourless" state, with the same being true of anti-colours. Furthermore, quarks within hadrons 
can change colours by exchanging gluons, which carry colour-anti-colour charge.

In order for a particle to exist in free space, it is required that it be colourless, which is true of both fundamental particles, and composite states like hadrons. For example, a meson composed of red-anti-red quarks can exist for some finite lifetime, while a single red quark cannot. In fact, if a quark is somehow liberated from a hadron - such as from a high-energy collision - it will hadronize effectively instantaneously with nearby matter, producing a cascade of hadrons which eventually decay to stable, non-coloured particles. The measured result of such a cascade is commonly known as a jet in particle physics, and the measurement of its energy and momentum can be critical for reconstructing and identifying rare physics processes.

A number of important mesons and baryons will be referenced in later sections, so their initial mention here may be useful. Perhaps most critically for this work, the proton is composed of three quarks - two up, one down - and has a charge of +1 . These three valence quarks are bound together by the exchange of gluons, as well as "sea" quark-antiquark pairs which appear and disappear instantly. Neutrons, similarly, are composed of two down quarks and an up quark, making them both neutral, and roughly as massive as the proton. Pions are mesons which come in charged and uncharged varieties, with $\pi^{+}\left(\pi^{-}\right)$composed of $u \bar{d}(d \bar{u})$, and $\pi^{0}$ composed of $u \bar{u}$ and $d \bar{d}$ states. Neutral pions, in particular, tend to decay frequently to pairs of photons, making them useful for understanding the energy measurement properties of particle detectors. Another class of meson which will be of relevance to later sections is the $B$-meson, which can be broadly defined as any meson containing a bottom quark. Due to the relatively large mass of the $b$-quark, $B$-mesons feature prominently in both Higgs boson production and decay (as explained further in Section 2.3), and tend to 
have both long lifetimes, and a greater degree of fragmentation during hadronization, which requires specialized reconstruction algorithms [20].

The sea quarks, valence quarks, and gluons which keep baryons together are known as partons. The theoretical modelling of how these components interact within a baryon can have a non-trivial effect on the predicted physics of a process. To provide a mathematical description of the motion of the partons within a baryon, the parton distribution function (PDF) is used. Practically speaking, PDFs characterize the probability density $f(x)$ for finding a parton with momentum $x$ at some energy scale, and for collider experiments in particular, tend to be derived in terms of the fraction of longitudinal momentum, i.e. the momentum component parallel to the collision axis. Due to the general difficulty of computing these distributions using lattice QCD, the derivation of PDFs is usually done empirically. In the context of collider physics, predictions can be obtained by fitting observables describing the behaviour of protons at varying energies to experimental data provided by fixed-target experiments such as BCDMS and NMC, as well as collider experiments such as CDF and DØ at the Tevatron, and ATLAS and CMS at the LHC [21].

\subsubsection{Conservation laws}

Beyond the obvious conservation laws in energy, momentum, and charge, a number of other conservation laws exist in the Standard Model, which dictate the allowed forms of interaction between particles. Analogous to electromagnetic charge conservation, the colour charge of all particles must balance for strong force-mediated interactions. Similarly, the number of leptons and quarks on either side of an interaction must be conserved, as well as the number of baryons. Some conservation laws are absolute, 
while others have exceptions. For example, flavour conservation is observed in strong nuclear interactions, while it is violated in weak nuclear interactions.

A particular conservation law worth discussing relates to the chirality of a particle, which can be loosely interpreted as the direction a particle spins, or its "handedness" 2. Chirality is tied into the law of parity conservation, which dictates, for example, that the physics of a system should remain the same if one transforms position as $(x, y, z) \rightarrow(-x,-y,-z)$, or similarly, inverts the "handedness" of each particle. Parity is conserved in the electromagnetic and strong interactions, and can be combined with charge conservation to form a compound symmetry of charge-parity (or $\mathcal{C P}$ ) conservation.

One notable place where parity conservation does not exist in the SM is within the weak nuclear force, as the weak bosons only couple to left-handed fermions, and right-handed anti-fermions, termed parity violation. The underlying reason for this behaviour is not precisely known, but an important result of parity violation in the SM is that all neutrinos are left-handed, while all anti-neutrinos are right-handed. In a more mathematically formal way, left-handed fermions are represented by doublet states, while right-handed fermions are represented by singlets, e.g. for the firstgeneration fermions [7],

$$
E_{L}=\left(\begin{array}{c}
e \\
\nu_{e}
\end{array}\right)_{L}, \quad e_{R}, \quad Q_{L}=\left(\begin{array}{c}
u \\
d
\end{array}\right)_{L}, \quad u_{R}, d_{R}
$$

\footnotetext{
${ }^{2}$ For massless particles, chirality is equivalent to helicity, or the sign of the projection of a particle's spin onto its momentum.
} 


\subsection{The SM Lagrangian}

Within the field theory framework, the dynamics and interactions of fermions and gauge bosons are described by gauge theories. One may recall from classical mechanics that the dynamics and forces of a system are described using a Lagrangian of the form $L=T-V$, where $T$ and $V$ are the kinetic and potential energies of the system. Analogous to classical mechanics, a gauge theory is described by a field theory Lagrangian. For example, a theory describing a scalar (spin-zero) particle $\phi$ inside of a potential $V(\phi)$ can be written as [22],

$$
\mathcal{L}=\frac{1}{2} \partial_{\mu} \phi \partial^{\mu} \phi-V(\phi)
$$

where the first term describes the motion of the scalar $\phi$, and the second term describes the interaction with the potential $V$. Generally speaking, gauge bosons are represented in QFT by gauge fields, which are four or more dimensional tensors, while fermions are represented by vectorial spinors. The SM Lagrangian is undoubtedly more complex than the above example, and describes the interactions between the gauge boson fields (represented symbolically by $W^{ \pm}, Z, G$, and the photon $A$ ) and the fermions. To first highlight the gauge field terms, the SM Lagrangian can be expressed as,

$$
\mathcal{L}_{\mathrm{SM}}=-\frac{1}{4} G_{\mu \nu}^{a} G_{a}^{\mu \nu}-\frac{1}{4} W_{\mu \nu}^{a} W_{a}^{\mu \nu}-\frac{1}{4} B_{\mu \nu} B^{\mu \nu}+(\text { fermion terms }) .
$$

Here, the first term describes an octet of gluon fields $G_{\mu}^{1, \ldots, 8}$, while $W_{\mu}^{1,2,3}$ and $B_{\mu \nu}$ correspond to fields representing a unified electroweak (EW) force.

Depending on how a field theory Lagrangian is constructed, it will respect certain 
symmetries, meaning that the behaviour of the quantum system is invariant under particular transformations. Symmetries can be either global or local; a global symmetry holds at all points in space-time for a given system, while a local (or gauge) symmetry varies from point to point. The local gauge symmetries of the SM are described by the unitary product group $\mathrm{SU}(3)_{C} \times \mathrm{SU}(2)_{L} \times \mathrm{U}(1)_{Y}$. Here, $S U(3)_{C}$ describes the symmetries associated to the strong force, where $C$ is the colour charge. The unitary product group $\mathrm{SU}(2)_{L} \times \mathrm{U}(1)_{Y}$ represents the symmetries of the electroweak force, where $L$ denotes left-handed chirality, and $Y$ is the weak hyper-charge.

A particular constraint of local symmetries is that massless vector fields are required for each symmetry generator [7]. This requirement poses a clear problem for the SM Lagrangian as written in Equation 2.3, as it implies that all fermions and gauge bosons must be massless, which we know to be untrue through decades of experimental validation. Therefore, in order to provide a complete theoretical description, the SM needs a final piece to introduce massive particles to the model.

\subsection{The Higgs mechanism}

The elusive key to massive gauge bosons and fermions lies in the Higgs mechanism, which combines the SM gauge theory with an additional field that spontaneously breaks the $\mathrm{SU}(2)_{L} \times \mathrm{U}(1)_{Y}$ symmetry. The work of introducing massive gauge bosons and fermions can be loosely broken up into three sectors. Within the electroweak sector, the Higgs mechanism explains how the three gauge bosons $W^{ \pm}$and $Z$ can obtain masses, but the photon remains massless. Although one can introduce mass terms of the form $-m_{q} \bar{\psi} \psi$ for quarks in an SU(3) gauge invariant manner [22], such a construction would also violate the isospin symmetry of the $\mathrm{SU}(2) \times \mathrm{U}(1)$ group (not 
to mention we also require massive leptons). Therefore, the Higgs mechanism is also necessary to properly introduce mass terms to the entire fermion sector of the SM. Lastly, the Higgs sector describes the interactions of the Higgs boson with the other sectors, along with its self-interactions, and mass generation.

\subsubsection{Electroweak sector}

To evaluate the effects of the Higgs mechanism on the electroweak gauge bosons, one can focus exclusively on these components of the SM Lagrangian,

$$
\mathcal{L}_{\mathrm{EW}}=-\frac{1}{4}\left(W_{\mu \nu}^{a} W_{a}^{\mu \nu}+B_{\mu \nu} B^{\mu \nu}\right)
$$

Here, the introduction of massive $W^{ \pm}$and $Z$ bosons begins with the addition of a complex SU(2) doublet of scalar fields,

$$
\Phi=\left(\begin{array}{c}
\phi^{+} \\
\phi^{0}
\end{array}\right) .
$$

With the introduction of this new scalar field, additional terms must be included in the Lagrangian to characterize its dynamics. In particular, the new scalar field terms will contain a kinematic term $\left|D^{\mu} \Phi\right|^{2}$, plus a new field potential controlled by parameters $\mu$ and $\lambda$,

$$
\mathcal{L}_{S}=\left|D_{\mu} \Phi\right|^{2}-V(\Phi)=\left(D^{\mu} \Phi\right)^{\dagger}\left(D_{\mu} \Phi\right)-\mu^{2} \Phi^{\dagger} \Phi-\lambda\left(\Phi^{\dagger} \Phi\right)^{2}
$$

The term $D_{\mu}$ is known as the covariant derivative, which is proportional to the electroweak gauge fields $B_{\mu}$ and $W_{\mu}^{\nu}$. Worth noting is that for $\mu^{2}<0$, the shape of $V(\Phi)$ 


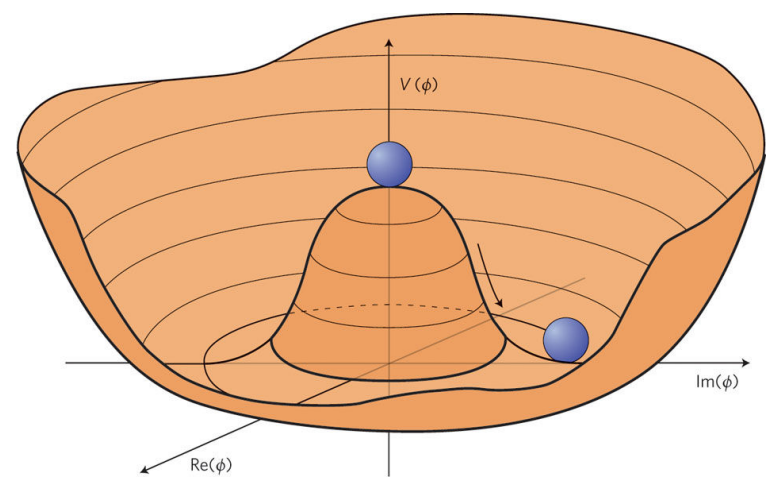

Figure 2.1: The potential $V(\Phi)$ introduced to the electroweak Lagrangian by the Higgs mechanism, under the condition $\mu^{2}<0$ [23]. The rolling ball represents the development of a vacuum expectation value (VEV) by the neutral component of the scalar doublet $\Phi$, induced by the introduction of a new minimum energy state by the potential.

will take the form of the potential shown in Figure 2.1 [23]. Generally speaking, it is also required that $\lambda>0$ in order to have a stable vacuum. Under these conditions, the neutral component of $\Phi$ develops a vacuum expectation value (VEV), defined as [22],

$$
\langle\Phi\rangle_{0}=\langle 0|\Phi| 0\rangle=\left(\begin{array}{c}
0 \\
v / \sqrt{2}
\end{array}\right), \quad \text { where } v=\sqrt{-\frac{\mu^{2}}{\lambda}} .
$$

From here, it is possible to apply a gauge transformation to $\Phi$ which redefines the field in terms of $v$ and a new field $H(x)$, bringing it to the so-called "unitarity gauge",

$$
\Phi(x) \rightarrow \frac{1}{\sqrt{2}}\left(\begin{array}{c}
0 \\
v+H(x)
\end{array}\right) .
$$

with the transformed $\Phi(x)$ being substituted back into the Lagrangian $\mathcal{L}_{S}$. This transformation induces spontaneous symmetry breaking, in which the $\mathrm{SU}(2)_{L} \times \mathrm{U}(1)_{Y}$ symmetry describing the heretofore unified electroweak force is broken. Using the transformed $\Phi(x)$, the kinematic derivative term $\left|D_{\mu} \Phi\right|^{2}$ resolves to [22], 


$$
\left|D_{\mu} \Phi\right|^{2}=\frac{1}{2}\left(\partial_{\mu} H\right)^{2}+\frac{1}{8} g_{2}^{2}(v+H)^{2}\left|W_{\mu}^{1}+i W_{\mu}^{2}\right|^{2}+\frac{1}{8}(v+H)^{2}\left|g_{2} W_{\mu}^{3}-g_{1} B_{\mu}\right|^{2} .
$$

The field terms $W_{\mu}^{1,2,3}$ and $B_{\mu}$ are components of the familiar unified electroweak fields, while the term $(1 / 2)\left(\partial_{\mu} H\right)^{2}$ represents a kinematic term for the Higgs field, which will be expanded upon in Section 2.3.3. In order to put this derivative expression in a form which is more familiar to the phenomenology laid out in Section 2.1, it is possible to rewrite the non-Higgs-coupled field terms above as [22],

$$
W^{ \pm}=\frac{1}{\sqrt{2}}\left(W_{\mu}^{1} \mp i W_{\mu}^{2}\right), \quad Z_{\mu}=\frac{g_{2} W_{\mu}^{3}-g_{1} B_{\mu}}{\sqrt{g_{2}^{2}+g_{1}^{2}}}, \quad A_{\mu}=\frac{g_{2} W_{\mu}^{3}+g_{1} B_{\mu}}{\sqrt{g_{2}^{2}+g_{1}^{2}}} .
$$

By substituting the above expressions back into the derivative term of Equation 2.9, and picking up the terms which are bilinear in the fields $W^{ \pm}, Z$, and $A$, mass terms are found for the $W^{ \pm}$and $Z$,

$$
m_{W}=\frac{1}{2} v g_{2}, \quad m_{Z}=\frac{1}{2} v \sqrt{g_{2}^{2}+g_{1}^{2}}, \quad m_{A}=0 .
$$

Worth noting is that the $\mathrm{U}(1)_{Y}$ symmetry remains unbroken by the mechanism, so the gauge field $A$, corresponding to the photon, has no mass term, as required. While the masses of the $W$ and $Z$ bosons are not directly predicted by the Standard Model, the ratio of the two can be defined analytically using a parameter $\rho$,

$$
\rho=\frac{m_{W}^{2}}{c_{W}^{2} m_{Z}^{2}},
$$

where the term $c_{W}$ is the Weinberg angle, which is related to the coupling strengths 
of the $W$ and $Z$ bosons as follows,

$$
c_{W}^{2}=1-\sin ^{2} \theta_{W}=\frac{g_{1}^{2}}{g_{1}^{2}+g_{2}^{2}}
$$

In the Standard Model specifically, $\rho=1$, an outcome that has been validated experimentally using precision measurements of $\theta_{W}[24,25,26], m_{W}[27,28]$, and $m_{Z}[29]$.

\subsubsection{Fermion sector}

Similar to the gauge bosons, fermion mass terms can be introduced to the SM using the scalar doublet field $\Phi$ defined in Section 2.3.1. The fermionic component of the SM Lagrangian can be written as,

$$
\begin{aligned}
\mathcal{L}_{\mathrm{F}}=\bar{L}_{i} D_{\mu} \gamma^{\mu} L_{i}+\bar{\ell}_{R i} i D_{\mu} \gamma^{\mu} \ell_{R i}+\bar{Q}_{i} i D_{\mu} \gamma^{\mu} Q_{i} & \\
& +\bar{u}_{R i} i D_{\mu} \gamma^{\mu} u_{R i}+\bar{d}_{R i} i D_{\mu} \gamma^{\mu} d_{R i} .
\end{aligned}
$$

The first two terms in this expression represent the left-handed lepton doublets $\left(L_{\ell}\right)$ and right-handed lepton singlets $\left(\ell_{R}\right)$, while the latter three terms correspond to the left-handed quarks, and right-handed up and down quarks, respectively. Furthermore, the term $\gamma^{\mu}$ represents the Dirac matrices, and the index $i$ runs over the three generations of fermions and quarks. By introducing the doublet $\Phi$ to the SM Lagrangian, the fermion-related components resolve to an $\mathrm{SU}(2)_{L} \times \mathrm{U}(1)_{Y}$ invariant Yukawa Lagrangian of the form [22],

$$
\mathcal{L}_{F}=-\lambda_{e} \bar{L} \Phi e_{R}-\lambda_{d} \bar{Q} \Phi d_{R}-\lambda_{u} \bar{Q} \tilde{\Phi} u_{R}+\text { (Hermitian conjugates) }
$$


In this expression, the $\lambda_{i}$ terms are coupling parameters, with the $\Phi$ terms surrounded by left-handed doublets and right-handed singlets, as previously established in Section 2.1, and $\tilde{\Phi}=i \tau_{2} \Phi^{*}$, where $\tau_{2}$ is the Pauli matrix,

$$
\tau_{2}=\left(\begin{array}{cc}
0 & -i \\
i & 0
\end{array}\right) .
$$

Using the gauge transformed definition of $\Phi$, it is possible to extract mass terms from the above expression for each fermion generation. Looking at the electron, for example, its component of the Yukawa Lagrangian resolves to [22],

$$
\mathcal{L}_{F} \supset-\frac{1}{\sqrt{2}} \lambda_{e}(v+H) \bar{e}_{L} e_{R}+\ldots
$$

Since $v$ and $\lambda_{e}$ are constants, then the product of these terms and $\bar{e}_{L} e_{R}$ yields the electron mass. A similar approach can be taken for each fermion generation to obtain,

$$
m_{\ell}=\frac{\lambda_{\ell} v}{\sqrt{2}}, \quad m_{q}=\frac{\lambda_{q} v}{\sqrt{2}} .
$$

\subsubsection{Higgs sector}

With the Higgs mechanism now embedded in the SM Lagrangian, the final consideration is how the Higgs boson itself originates and self-couples. Much the same as the other massive particles in the model, the kinematic term for the Higgs boson exists in the expansion of $\left|D_{\mu} \Phi\right|^{2}$, while the mass and self-interaction components come from the scalar potential,

$$
V(\Phi)=\mu^{2} \Phi^{\dagger} \Phi+\lambda\left(\Phi^{\dagger} \Phi\right)^{2} .
$$


By substituting the transformed $\Phi(x)$, and defining $v^{2}=-\mu^{2} / \lambda$, then the scalar potential resolves to,

$$
V=-\frac{1}{2} \lambda v^{2}(v+H)^{2}+\frac{1}{4} \lambda(v+H)^{4} .
$$

Furthermore, the Lagrangian describing the Higgs boson can be written as,

$$
\mathcal{L}_{H}=\frac{1}{2}\left(\partial_{\mu} H\right)^{2}-\lambda v^{2} H^{2}-\lambda v H^{3}-\frac{\lambda}{4} H^{4} .
$$

From this form of the Lagrangian, it is evident that several types of Higgs boson self-interactions are possible - namely, annihilation/pair production, triple gauge couplings, and $2 \rightarrow 4$ scattering of four Higgs bosons. Furthermore, the Higgs boson mass is defined in terms of the parameter $\mu$ as $M_{H}=\sqrt{2 \lambda} v=\sqrt{-2 \mu^{2}}$. Worth noting is that the parameter $\mu$ (hence, $M_{H}$ ) must be chosen for predictions to be made on the rates of production and decay of the Higgs boson. Ultimately, it was this necessity of choice that precipitated the decades-long experimental search for the Higgs boson and its mass.

\subsection{Scattering amplitudes and corrections}

The ultimate goal of a field theory is to be predictive, allowing for the calculation of scattering cross sections and branching ratios. When considering the interaction of two or more particles, the cross section can be thought of as an area transverse to the relative motion of the particles, within which they must meet to cause a scattering interaction. Accordingly, the cross section for a process is proportional to the probability of a given interaction occurring (or the scattering amplitude), such that 


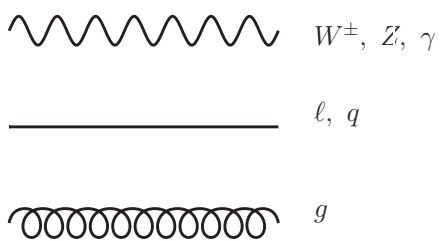

(a) Particle propagators

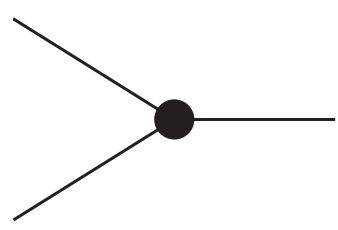

(b) Interaction vertices

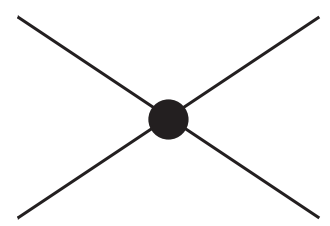

Figure 2.2: The fundamental components of Feynman diagrams, particle propagators and interaction vertices.

interactions with large cross sections will occur more frequently than those with small cross sections. In experimental particle physics, cross sections are usually expressed in terms of barns $[\mathrm{b}]$, where $1 \mathrm{~b}=10^{-28} \mathrm{~m}^{2}$. Branching ratios characterize the probability for an unstable particle to decay to some final state, and are typically unitless, expressed as a fraction of the total width of the particle, $\Gamma$. The total width is conventionally reported in units of energy, owing to the uncertainty relation $\Delta E \Delta \tau \geq \hbar / 2$, meaning that the uncertainty on the lifetime $(\tau)$ of an unstable particle is re-expressed as a "width" around its central mass value.

In perturbation theory, probability amplitudes are expressed in the form of expectation values, written generically as $\langle\psi|\mathcal{O}| \psi\rangle$, where $|\psi\rangle$ describes the state of the system, and $\mathcal{O}$ represents some operator describing a physical property of the system, such as energy or momentum. The state vector $|0\rangle$, in particular, represents the ground (or vacuum) state of the system. Wick's theorem [30] demonstrates that an expectation value of the form,

$$
\left\langle 0\left|T\left\{\phi_{I}\left(x_{1}\right) \phi_{I}\left(x_{2}\right) \ldots \phi_{I}\left(x_{n}\right)\right\}\right| 0\right\rangle,
$$

containing a number of time-ordered interaction operators $\phi_{I}$ can be expressed as a sum of products of Feynman propagators, which can be understood as self-contained 

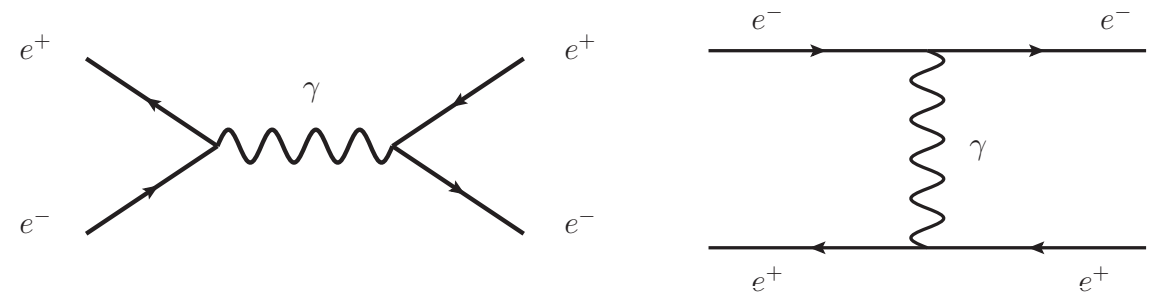

Figure 2.3: Feynman diagrams for a tree-level, photon-mediated $e^{+} e^{-} \rightarrow e^{+} e^{-}$process.

expectation values describing the probability amplitude for a particle to travel some distance with time, or travel with some energy and momentum.

The great revelation of this theorem is that it allows for a series of Feynman propagators - and more importantly, particle interactions - to be expressed pictorially using Feynman diagrams. In particular, a Feynman diagram is a time-ordered representation of a scattering process, wherein particle propagators, which are represented as lines, meet and cause interactions, represented using vertices, as shown in Figure 2.2. For example, an electron-positron scattering process (known as "Bhabha scattering" [7]) mediated through quantum electrodynamics (QED) can be drawn using the diagrams in Figure 2.3. In this Feynman diagram, an electron and positron meet and annihilate, causing the production of a photon, which later undergoes pair production, creating a final state electron-positron pair. Worth noting, as well, is that time flows rightward, meaning that the positrons go "backward" in time.

The scattering amplitude for a given process is typically denoted by $\mathcal{M}$, such that the cross section is proportional to $\mathcal{M}^{*} \mathcal{M}$, or $|\mathcal{M}|^{2}$, the matrix element. In the notation of QFT, the scattering amplitude for the full $e^{+} e^{-} \rightarrow e^{+} e^{-}$process may be expressed as [7],

$$
\mathcal{M} \sim\left\langle e^{+} e^{-}\left|H_{I}\right| \gamma\right\rangle^{\mu}\left\langle\gamma\left|H_{I}\right| e^{+} e^{-}\right\rangle_{\mu}
$$




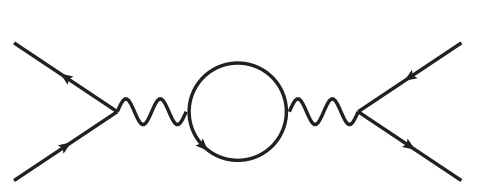

(a) Virtual loop

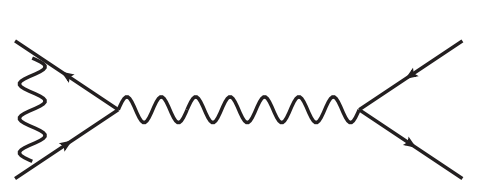

(b) Virtual emission

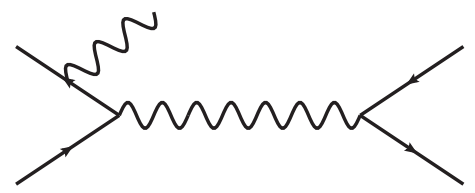

(c) Real emission

Figure 2.4: Possible corrections to the tree-level $e^{+} e^{-} \rightarrow e^{+} e^{-}$scattering process.

where $H_{I}$ denotes an operator describing the $e^{+} e^{-} \gamma$ interaction vertex, and the amplitude sums over a set of indices $\mu$, which denote the different possible physical configurations of the system, such as spin or chirality. In general, the scattering amplitude for a process is a linear sum of the amplitudes of all possible Feynman diagrams. Therefore, the matrix element $|\mathcal{M}|^{2}$ not only contains the moduli of individual diagrams, but also interference terms between diagrams, which may induce both negative and positive changes in the cross section.

The diagram in Figure 2.3 is also an example of a leading order (LO) or tree-level process. Tree-level diagrams are constructed using the least number of particle lines and vertices possible to represent the full scattering process. In general, however, a scattering process is defined by both tree-level contributions, and diagrams with corrections which represent higher-order terms in the perturbative expansion. Corrections may take the form of particle loops, as well as radiation from internal or external particle lines, and may be real (producing additional initial or final state particles) or virtual (producing no additional particles).

Returning to the Bhabha scattering process, examples of diagrams with higherorder corrections may appear as in Figure 2.4. Such diagrams would be considered next-to-leading-order (NLO) corrections to the tree-level process. Similarly, diagrams representing even higher order next-to-next-to-leading-order (NNLO), $\mathrm{N}^{3} \mathrm{LO}$, etc. cor- 


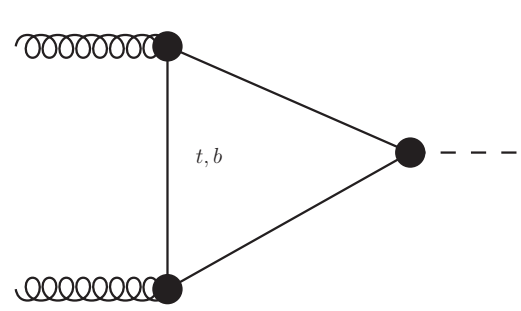

(a) Gluon-gluon fusion

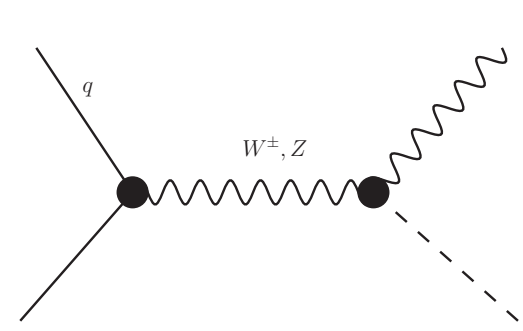

(c) $W^{ \pm} / Z$ associated production

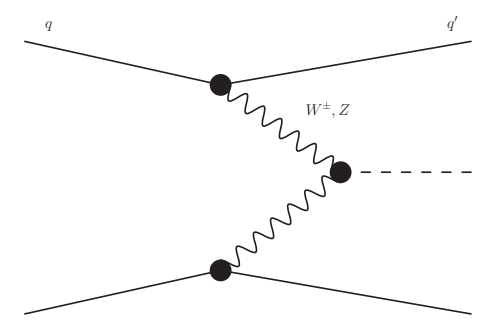

(b) Vector boson fusion

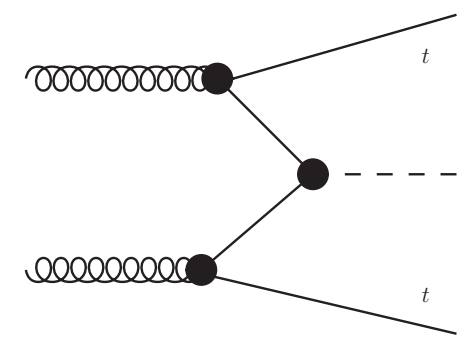

(d) $t \bar{t}$ associated production

Figure 2.5: Tree-level Feynman diagrams of the four main Higgs boson production mechanisms at the LHC.

rections are possible, which include further loops and emissions. In practice, however, the change in the process cross section tends to shrink asymptotically to zero as more corrective terms are added, meaning that sensible cross section predictions are often possible at NLO or NNLO. Worth noting, as well, is that Feynman diagrams can contain higher-order corrections in both QED and QCD, depending on whether quarks/gluons are involved in the process.

\subsection{Higgs boson production and decay modes}

Higgs boson production at the LHC occurs through proton-proton (or $p p$ ) collisions. Recalling that the proton is composed of three valence quarks bound together by a "sea" of gluons and virtual quark/anti-quark pairs, a $p p$ collision can involve the 


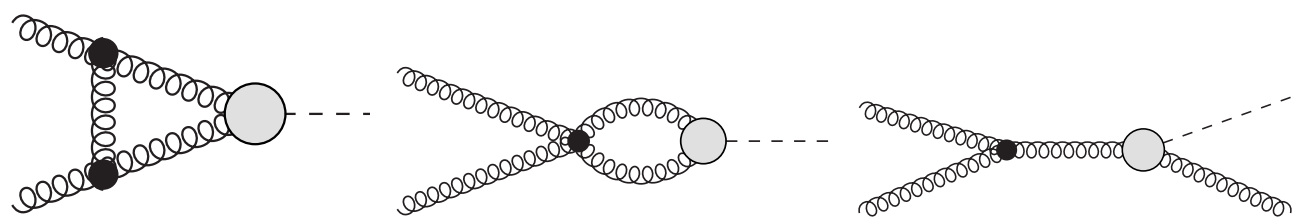

Figure 2.6: Examples of NLO QCD corrections to the ggF Higgs boson production process. For simplicity, the $t \bar{t}$ loop has been enclosed in a filled circle.

interaction of gluons with gluons, quarks with quarks, or quarks with gluons. The prevalence of a given type of Higgs boson production process is given by its cross section, which at the LHC is typically expressed in picobarns $\left(\mathrm{pb}\right.$, or $\left.10^{-40} \mathrm{~m}^{2}\right)$. The four highest production cross sections, in descending order, are: gluon fusion (ggF); vector boson fusion (VBF); weak boson-associated production (VH); and top-anti-topassociated production (ttH). The tree-level Feynman diagrams for each production process are shown in Figure 2.5.

Gluon fusion, as the name implies, involves the interaction of two gluons to form a Higgs boson. In particular, the process proceeds through loops of heavy quarkanti-quark pairs, predominantly $t \bar{t}$ and $b \bar{b}$. These particular quark loops dominate the interaction because the coupling strength of a given particle to the Higgs boson is proportional to its mass. Therefore, more massive particles will couple more strongly to the Higgs boson, while massless particles (photons and gluons) will not couple directly at all. Vector boson fusion proceeds through the inverse decay of $W^{ \pm}$or $Z$ bosons which are exchanged by quarks. Associated Higgs boson production encompasses two interactions: $W^{ \pm} / Z$ associated production occurs when a $q \rightarrow V q^{\prime}$ interaction leads to the emission of a Higgs boson from the intermediate weak boson; and "top fusion" occurs when two colliding gluons convert to top-anti-top pairs, with one $t$ and $\bar{t}$ from each conversion forming a Higgs boson. In the case of $W^{ \pm} / Z$ associ- 

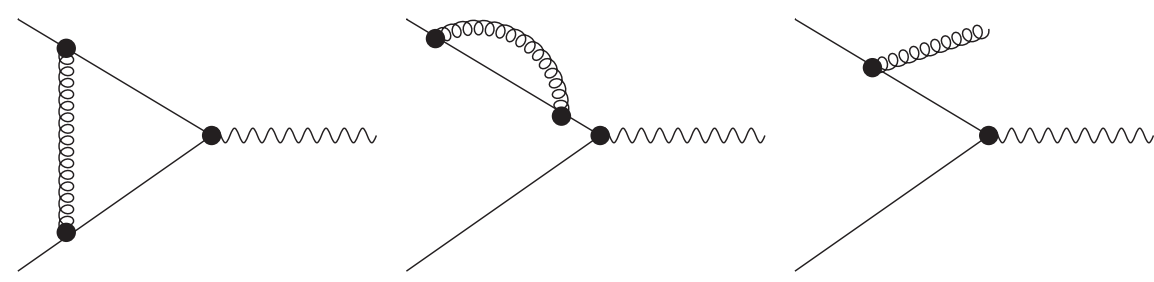

Figure 2.7: Examples of corrections to the vertices involved in NLO QCD VBF and VH Higgs boson production calculations [22].

ated production, the intermediate weak boson may decay hadronically (i.e. $V \rightarrow q q^{\prime}$ ) or leptonically (i.e. $Z \rightarrow \ell^{+} \ell^{-}$or $W^{ \pm} \rightarrow \ell^{+} \nu\left(\ell^{-} \bar{\nu}\right)$ ).

Worth noting is that ggF Higgs boson production, which is an entirely strong force-mediated process, receives important higher-order corrections in QCD, which can significantly affect both the production cross section and process kinematics. A representative example of some of the real and virtual NLO QCD corrections to the tree-level ggF process are given in Figure 2.6. In particular, the best-known $\mathrm{N}^{3} \mathrm{LO}$ QCD corrections to the process yield a total cross section which is $\sim 300 \%$ higher than the tree-level prediction [31]. The impact of these corrections on the description of the ggF process is explored further in Sections 5.1 and 8.2.1. In contrast, electroweak corrections to the ggF process are much smaller at $\mathcal{O}(5 \%)$ [32]. Being purely electroweak processes at tree-level, the effects of higher-order QCD corrections for VBF and VH production are less significant than for ggF; the NNLO QCD corrections for the VBF process are $\mathcal{O}(10 \%)$ [33, 34], while for VH they are $\mathcal{O}(40 \%)$ [35]. Examples of NLO QCD corrections to the $q q V$ vertices involved in both VBF and VH production are shown in Figure 2.7. Likewise, EW corrections are small at $\mathcal{O}(10 \%)$ for both VBF [36] and VH production [37].

The Higgs boson is a comparatively heavy particle, and consequently tends to 
decay almost immediately into lighter, more stable particles, with an extremely short lifetime of $\sim 10^{-22} \mathrm{~s}[38]$. The proportionality of the Higgs boson couplings to mass is reflected in the branching ratios of the various final states. In particular, final states with heavy particles, or interactions mediated by heavy particles, tend to dominate the Higgs boson total width, while modes with direct couplings to lighter particles are extremely rare. Tree-level Feynman diagrams for a number of representative decay processes are shown in Figure 2.8, whose structures can be broadly grouped into three categories: direct Higgs couplings to massive particles (e.g. $H \rightarrow b \bar{b}$ ); loop-induced decays to massless particles (e.g. $H \rightarrow \gamma \gamma$ ); and decays to leptons through pairs of weak bosons (e.g. $H \rightarrow Z Z^{*} \rightarrow \ell^{+} \ell^{-} \ell^{+} \ell^{-}$or $\left.H \rightarrow W W^{*} \rightarrow \ell^{+} \nu \ell^{-} \bar{\nu}\right)$. The branching ratios of these decays are also sensitive to the mass of the Higgs boson, with theoretical predictions for the branching ratios of each possible Higgs boson decay mode shown in Figure 2.10 as a function of the Higgs boson mass.

The focus of this work is the $H \rightarrow Z Z^{*} \rightarrow \ell^{+} \ell^{-} \ell^{+} \ell^{-}$(or simply "4 $\ell$ ") decay, in which the Higgs boson decays into two intermediary $Z$ bosons: one on-shell boson, which is a real particle with a mass centred around the $Z$ boson mass peak of $m_{Z}=$ 91.1876 GeV [39]; and one off-shell boson, which is a virtual particle, whose mass and width are not fixed around a central point. Rather, the mass distribution of the offshell boson is a spectrum which is mainly contained in the region of $m_{Z^{*}}<20 \mathrm{GeV}$. These intermediary weak bosons decay to pairs of opposite sign leptons, with four final states under consideration, listed by descending branching ratio: $4 \mu, 2 \mu 2 e, 2 e 2 \mu$, and 4e. In principle, decays to pairs of $\tau$ leptons are also possible, however this experimental signature tends to contain hadronic activity and invisible decay products (neutrinos), and is therefore more difficult to reconstruct experimentally. Accordingly, 


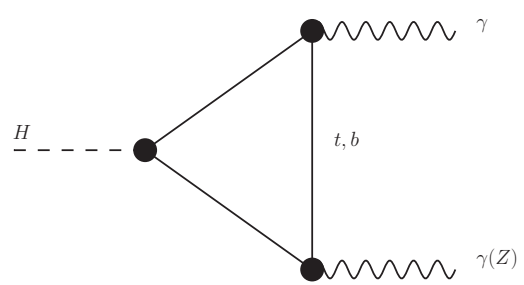

(a) $H \rightarrow \gamma \gamma(Z)$ ( $q \bar{q}$ loop)

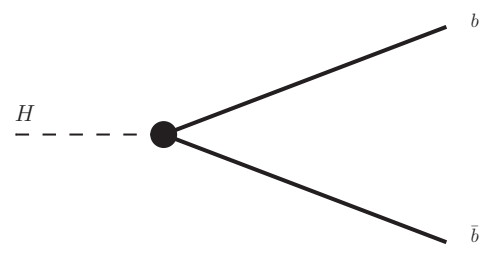

(c) $H \rightarrow b \bar{b}$

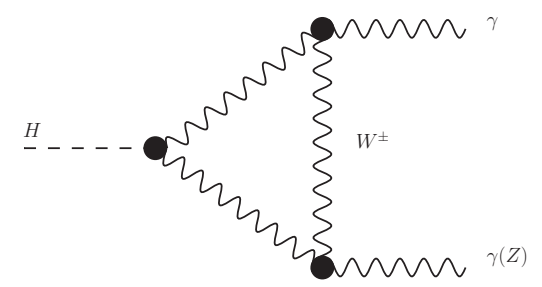

(b) $H \rightarrow \gamma \gamma(Z)(W$ loop)

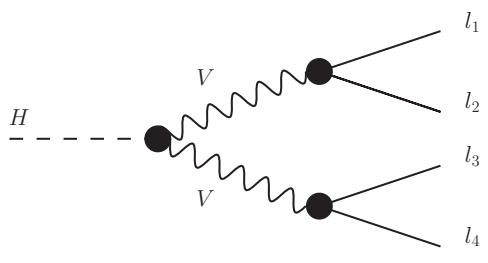

(d) $H \rightarrow V V^{*} \rightarrow 4 l$

Figure 2.8: Tree-level (leading order) Feynman diagrams for notable light-to-medium Higgs boson decay channels searched for at the LHC.

decay signatures with $\tau \tau$ final states are not considered in the following chapters.

The $H \rightarrow Z Z^{*} \rightarrow 4 \ell$ decay channel is considered the "golden channel" despite its small branching ratio $\left(\Gamma_{Z Z^{*}} \propto 10^{-4}\right)$ due to the fact that all final state leptons can be reconstructed fully, and with high experimental resolution, within both the ATLAS and CMS detectors. Furthermore, higher-order corrections to the $4 \ell$ Higgs decay process tend to be small around a region of $m_{H}=125 \mathrm{GeV}$, with QCD and EW corrections together leading to a change of $2-4 \%$ in the partial width $\Gamma_{Z Z^{*}}$, and affecting the final state lepton kinematics by at most 10\% [40]. Therefore, it can be said that the $H \rightarrow Z Z^{*} \rightarrow 4 \ell$ process is already well-understood using a leading order approach. Other decay processes such as $H \rightarrow \gamma \gamma$ which proceed at tree-level through heavy quark loops are more directly affected by higher-order corrections, however these final states are not under consideration in this work. 


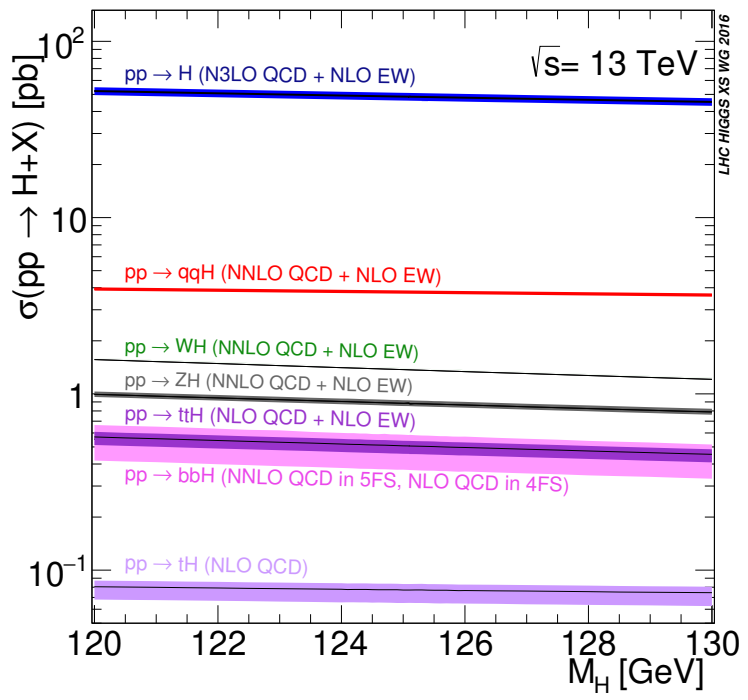

Figure 2.9: Predicted cross sections of various Higgs boson production methods at $\sqrt{s}=13 \mathrm{TeV}$ as a function of $m_{H}$, the Higgs boson mass [41].

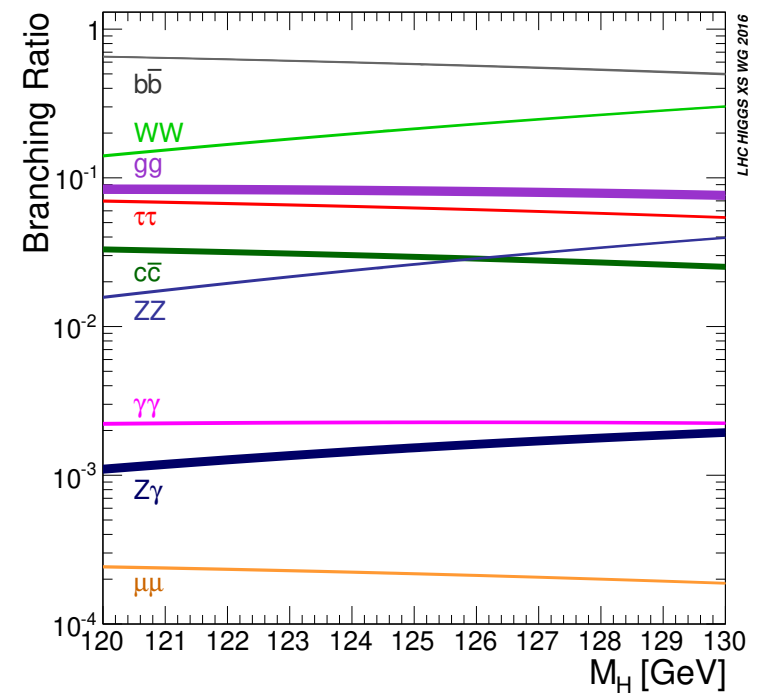

Figure 2.10: Predicted branching ratios of the various Higgs boson decay modes as a function of $m_{H}$, the Higgs boson mass [41]. 


\subsection{Beyond Standard Model Higgs physics}

While the Standard Model is indeed one of the most robustly tested and validated theories of nature, there are still a number of notable experimental observations which are unexplained by the SM, or conflict with its predictions or implications. Many of these anomalous observations have links to Higgs physics, and as such, are worth mentioning for their potential to be probed by ATLAS at the LHC. The following is a brief overview of some outstanding problems with the Standard Model to motivate the work of the following chapters.

Perhaps the most critical outstanding issue with the SM is the hierarchy problem, the central question of which is why the Higgs boson is so "light", when the existing Standard Model formulation seems to suggest it should be a very massive particle [42]. The heart of this problem is that the Higgs boson mass calculation in the SM Lagrangian receives significant corrections in the form of heavy particle loops, as shown in Figure 2.11, which should result in a mass that is much larger than the observed $m_{H} \sim 125 \mathrm{GeV}$. In order to predict a light Higgs boson, the parameters of the Standard Model have to be fine-tuned in a manner that negates these large contributions to the Higgs mass.

To remedy this issue, several BSM theories falling under the umbrella of Two Higgs Doublet Models - the most notable of which is supersymmetry [43] - introduce a second Higgs doublet during electroweak symmetry breaking. The use of two doublets leads to the presence of additional Higgs bosons, along with new particles (hence, new corrections) which cancel the problematic terms in the Higgs mass calculation. As a result of these changes, the theory allows for a light, SM-like Higgs boson plus one (or more) additional Higgs boson(s), which may feature non-SM behaviour, such 


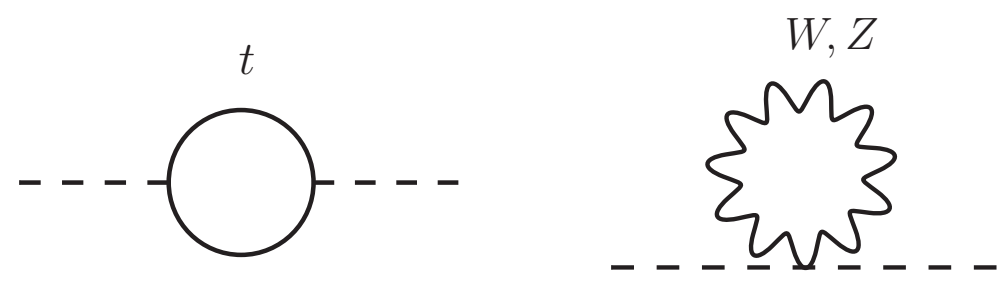

Figure 2.11: Corrections to the Higgs mass calculation which induce the hierarchy problem.

as $\mathcal{C P}$-odd or mixed-CP Higgs couplings. As of yet, however, no evidence of new, supersymmetric particles has been found at the LHC [44].

Theories falling under the umbrella of Little Higgs models also posit gauge structures which can produce a light Higgs boson with minimal new, non-SM contributions [45]. Such theories centre around a new strong force acting at an energy scale of tens of $\mathrm{TeV}$. The model may begin with a more complicated symmetry group than the SM (such as SU(5), for example) which has certain subgroups broken, or weakly gauged, with the latter meaning that the new strong force only acts weakly below its $\mathrm{TeV}$ energy scale. In such a model, the corrections necessary to maintain a light Higgs boson come from the introduction of new fermions and gauge bosons which emerge from the symmetry breaking. The Higgs boson itself may also be a composite particle whose internal interactions are mediated by the new strong force, allowing the Higgs boson to exhibit non-SM properties or behaviour, while the symmetries of the gauge theory are still allowed to break to the familiar SM result [45].

Another notable problem with the Standard Model is the fact that "observable" matter makes up only around $5 \%$ of the known universe [46]. The remaining contents of the universe are thought to be dark matter and dark energy, with dark matter (DM) in particular making up roughly $27 \%$ of the energy content of the universe [46]. This energy and matter is considered "dark" because it is not known to interact 
with observable matter. To this date, the exact nature of DM remains unknown, although several theories exist which introduce dark matter "candidates" that fit the description required by astrophysical observations. For example, one of the more common DM formulations is described in terms of Weakly Interacting Massive Particles (WIMPs). Candidates for WIMPs are postulated by several theories, including many supersymmetric [47, 48, 49] and Little Higgs models [45]. In the context of Higgs physics, a particle must interact with the Higgs field to acquire mass, so in principle it is possible to search for DM directly at colliders such as the LHC. To date, however, no dark matter signatures have been detected by either ATLAS or CMS [50].

Beyond direct searches for new particles, it is also possible to probe the Higgs boson itself to determine if its properties - such as spin, parity, and couplings are consistent with Standard Model expectations. For example, previous searches at $\sqrt{s}=8 \mathrm{TeV}$ by ATLAS have ruled out non-zero spin hypotheses quite definitively [51]. Constraints have also been made on the charge-parity of the Higgs boson which place it in agreement with SM expectations [51], however, they do not definitively rule out the presence of non-SM behaviour, such as $\mathcal{C P}$-mixing. Likewise, excesses in Higgs boson production rates have been previously observed in different Higgs boson decay channels using $\sqrt{s}=13 \mathrm{TeV}$ data $[52,53]$.

With these points in mind, it is worth re-investigating the properties of the Higgs boson at $\sqrt{s}=13 \mathrm{TeV}$ to determine what BSM scenarios may not be ruled out by the new data. For example, it is possible to probe the $\mathcal{C P}$ structure of the Higgs boson using its production and decay rates, as well as its kinematic properties. The presence of new, non-SM operators in the Lagrangian may also be indicated by deviations in 
the rate or kinematics of the Higgs boson, or the final state particles of its production and decay chain. New constraints on such properties may give an indication of a preferred direction for future searches, motivating a focus on particular classes of BSM models. 


\section{Chapter 3}

\section{The LHC and ATLAS}

The observation of a Higgs boson requires both a sizeable energy input to produce the particle, as well as an intricate detection system to observe its decay products. The Large Hadron Collider is able to produce millions of high-energy proton-proton collisions per second for this purpose (among others), although only a rare few will create a Higgs boson. Likewise, the ATLAS detector (A Toroidal LHC ApparatuS) was designed and built with the intention of picking out the decay products of these rare events from among the debris and electronic noise of the surrounding collisions. In this chapter, Section 3.1 provides an overview of the construction and collision methodology of the LHC, while Section 3.2 provides a detailed look at the function and components of the ATLAS detector, focusing particularly on the detector elements relevant to later chapters of this work. 


\subsection{The Large Hadron Collider}

The Large Hadron Collider (LHC) is a circular proton-proton collider, $27 \mathrm{~km}$ in circumference, located underground at CERN, along the French-Swiss border. The first proposal for the LHC was made at a workshop in Lausanne, Switzerland in 1984, with decades of work culminating in the collider becoming fully functional in 2009 . From 2009 to 2013, the collider was operated at a centre-of-mass energy of $\sqrt{s}=$ $7 \mathrm{TeV}$, or $3.5 \mathrm{TeV}$ per proton beam. A brief shutdown in 2011 allowed for an increase in centre-of-mass energy to $\sqrt{s}=8 \mathrm{TeV}$, with data taking occurring continually until early 2013. A shut down of the LHC in 2013 allowed for the application of several critical upgrades which prepared the collider for operation at higher centre-of-mass energies and luminosity. Proton-proton collisions resumed in summer 2015, with data acquisition ongoing at a centre-of-mass energy of $\sqrt{s}=13 \mathrm{TeV}$. In late 2018, the LHC will shut down once more for a second series of upgrades, with proton-proton collisions resuming in 2021 at the full design energy of $\sqrt{s}=14 \mathrm{TeV}$.

The production and acceleration of proton beams at the LHC is a four-stage process, and is shown pictorially in Figure 3.1. First, protons are obtained by stripping electrons from hydrogen atoms. These protons are then accelerated by the LINAC2 linear accelerator up to an energy of $50 \mathrm{MeV}$. The energy of the proton beams is then increased to $1.4 \mathrm{GeV}$ by travelling through the Proton Synchrotron Booster (PSB). Prior to entering the LHC, the protons are further accelerated by the Super Proton Synchrotron (SPS), bringing the beam energy further up to $450 \mathrm{GeV}$, at which point they are injected into the LHC main ring [54].

The main LHC ring is home to four experiments: ATLAS, ALICE, CMS, and LHCb. ATLAS [55] and CMS [56] are considered "sister" experiments, established 


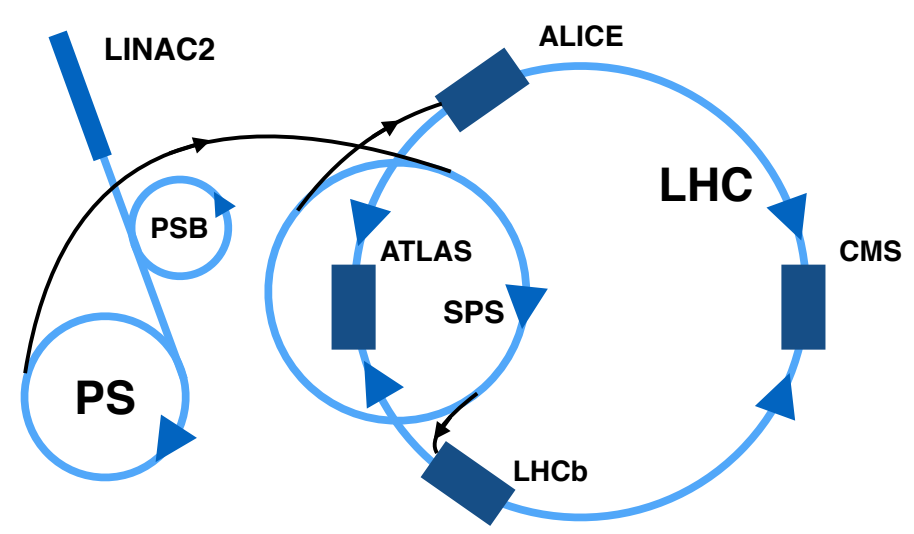

Figure 3.1: Diagram of the injection chain at the LHC for proton beams. The collider is designed to accelerate each proton beam up to energies of $7 \mathrm{TeV}$. The relative locations of the four main experiments are also labelled. Figure based on Ref. [54].

around general purpose, cylindrical detectors which focus on the measurement of the decay products from $p p$ collisions. The two experiments perform many complementary measurements and discovery searches, with perhaps the most notable result being the co-discovery of the Higgs boson in 2012 [1,2]. The ALICE experiment [57] is designed to study the decay products of lead ion collisions, with the underlying goal being the study of quark-gluon plasma. Lastly, the LHCb experiment [58] is dedicated to the study of charge-conjugation/parity violation and rare decays of heavy-flavour particles - namely, the bottom quark, from which the LHCb derives its name. Only results from the ATLAS detector are considered in this work.

\subsubsection{Collision terminology}

To better understand the dynamics of particle interactions within the ATLAS detector, it is useful to first define some terminology that describes the rate and intensity of $p p$ collisions produced by the LHC. Data acquisition is measured in units of lumi- 
nosity; in particular, the instantaneous luminosity $\mathcal{L}$ roughly describes the amount of data acquired at a given time, while the integrated luminosity,

$$
\int \mathcal{L}=\int \mathcal{L}(t) d t
$$

describes the total accumulated data for some time period. The instantaneous luminosity is defined in units of $\mathrm{s}^{-1} \mathrm{~m}^{-2}$, with the integrated luminosity defined in $\mathrm{m}^{-2}$. That $\int \mathcal{L}$ has units which are the inverse of the cross section is no coincidence; in fact, the estimated cross section of a given $p p \rightarrow X$ process is related to the integrated luminosity through the number of recorded events, $N_{p p \rightarrow X}$, as follows,

$$
\sigma_{p p \rightarrow X}=\frac{N_{\mathrm{pp} \rightarrow \mathrm{x}}}{\epsilon \int \mathcal{L}} .
$$

The additional term $\epsilon$ represents a generalized efficiency for the process, which can be affected by a number of factors, such as detector-related inefficiencies, and the fiducial coverage, i.e. the region in which one can "trust" the accuracy of measurements.

The definition of the instantaneous luminosity is slightly more complex [59],

$$
\mathcal{L}=\frac{n_{b} n_{1} n_{2} f_{r}}{2 \pi \Sigma_{x} \Sigma_{y}} .
$$

Here, the terms in the numerator and denominator of $\mathcal{L}$ relate to both the intensity and population of the proton beams, as well as their interaction cross sections. In the numerator, $n_{b}$ is the number of bunches per beam, $n_{i}$ is the number of protons within bunch $i$, and $f_{r}$ is known as the revolution frequency of the beams, or the rate at which the beams circle the collider ring. In the denominator, $\Sigma_{x}$ and $\Sigma_{y}$ are the horizontal and vertical convolved beam widths, respectively, which can be 
approximately understood as the length and width of the interaction surface shared by the colliding beams.

To further understand the nature of the interactions between protons at the LHC, one must consider that the protons do not "collide" in a classical sense, but rather interact through their constituent partons - namely, the quarks and gluons. In each collision, the partons which carry the largest fraction of momentum in each proton dictate the nature of the interaction, which is termed the hard scattering (HS) interaction. A number of secondary interactions can occur in addition to the HS interaction, forming what is called the underlying event (UE) $[60,61]$. An example of the UE is illustrated in Figure 3.2 for a $p p$ collision producing two final state partons. The UE can include particles originating from "beam-beam remnants", secondary collisions of incoming partons, as well as initial and final state radiation from the incoming and outgoing partons, respectively [60]. It is also possible for so-called multiple parton interactions (MPI) to contribute to the multiplicity of the final state, wherein low energy scattering interactions and hadronization of outgoing quarks produce closelygrouped cascades of particles (i.e. jets).

Of course, for any given recorded collision, there will inevitably be additional $p p$ collisions happening in close proximity, due to the fact that bunches of protons are collided, rather than individual particles. These collisions produce additional final state particles beyond those from the HS, UE, and MPI, and are referred to as pile-up interactions, owing to the fact that detector readout from the collision products overlaps with (or "piles up" against) signal from the HS interaction, creating unwanted noise that obscures the signal of interest. Two types of pile-up can occur for a given bunch crossing: In-time pile-up occurs within the same bunch crossing as 


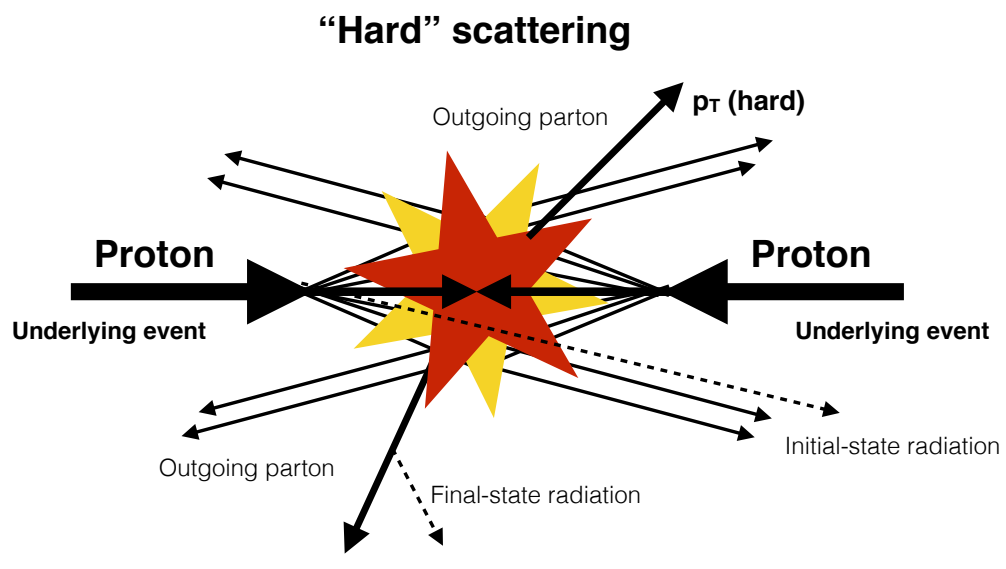

Figure 3.2: Illustration of the way a QCD Monte Carlo generator would simulate a proton-proton collision in which a hard $2 \rightarrow 2$ scattering process occurs, leading to final state partons with some momentum $p_{\mathrm{T}}$ transverse to the initial proton momenta (hard). Figure based on Ref. [60].

the HS interaction, typically at point displaced from the HS vertex; while out-of-time pile-up occurs at a bunch crossing different from the HS interaction. The amount of noise due to pile-up is directly related to the instantaneous luminosity expressed in Equation 3.3, in the sense that a higher luminosity implies more bunch crossings, hence more pile-up interactions. Given an instantaneous number of collisions per bunch crossing $\mu$, the amount of pile-up for a data taking period is quantified using the average number of interactions per bunch crossing, symbolized by $\langle\mu\rangle$.

\subsection{The ATLAS detector}

The ATLAS detector is a general purpose, cylindrical particle detector, designed to cover a broad reach of physics opportunities through measurement of the products of high energy $p p$ collisions at the LHC. A diagram of the ATLAS detector is shown in Figure 3.3. It is the largest detector on the LHC ring, standing at $25 \mathrm{~m}$ high, 44 


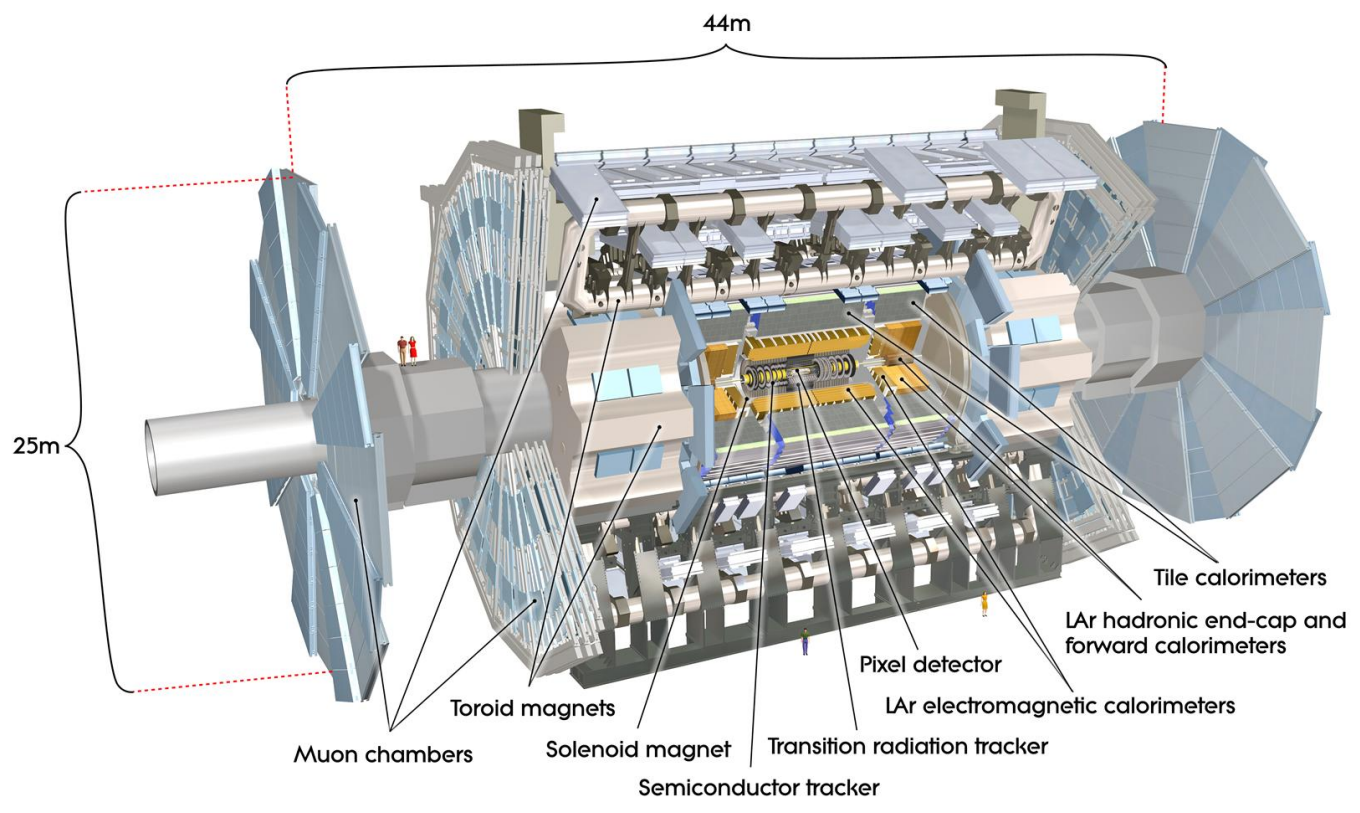

Figure 3.3: Cut-away view of the ATLAS detector. The detector stands $25 \mathrm{~m}$ high and is $44 \mathrm{~m}$ long, with a total weight of approximately 7000 tonnes [55].

m long, and weighing close to 7000 tonnes [55]. The ATLAS detector is comprised of multiple layered sub-detectors, with each tailored to measure some combination of energy, position, momentum, and charge. With this configuration, nearly $4 \pi$ coverage of active material is achieved around the $p p$ interaction point, which occurs at the geometric centre (or near the centre) of the detector. Such extensive coverage allows not only for detailed measurements of the outgoing particles from a given collision, but also the reconstruction of the underlying process which produced them.

The ATLAS coordinate system is fundamentally a right-handed cylindrical coordinate system, as demonstrated in Figure 3.4. The detector is oriented such that the $z$-axis is parallel to the $p p$ beam line, with the $x$-axis pointed towards the centre of the LHC ring, and the $y$-axis oriented accordingly to be perpendicular. Of particular 

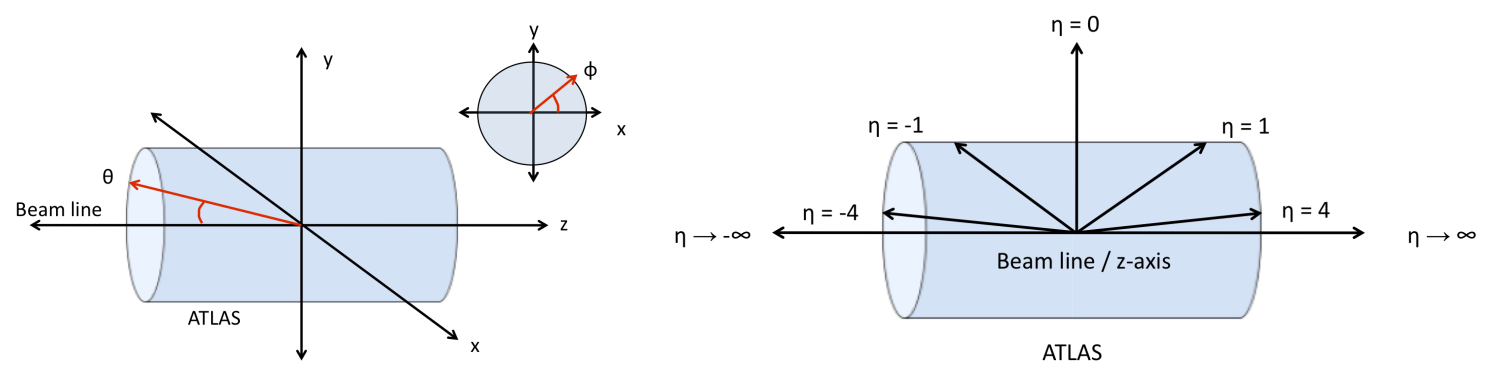

Figure 3.4: (Left) Layout of the ATLAS detector in Cartesian coordinates, with definitions of $\theta$ and $\phi$, relative to their rotational axes. (Right) Pseudorapidity and its relation to the $z$-axis.

note is that the angular displacement from the $z$-axis tends to be expressed in terms of rapidity, $y$, which is defined as,

$$
y=\frac{1}{2} \ln \left(\frac{E-p_{z}}{E+p_{z}}\right),
$$

where $E$ and $p_{z}$ are the energy and $z$-component of the momentum for a given particle, respectively. For highly relativistic particles with $E, p>>m$, the pseudorapidity, $\eta$, is also frequently used in place of the rapidity, and is defined as,

$$
\eta=-\ln \left(\tan \left(\frac{\theta}{2}\right)\right)
$$

where $\theta$ is the angle between the particle momentum and the beam line $/ z$-axis. For particles with negligibly small mass compared to their momentum, the rapidity and pseudorapidity will be functionally equivalent. Unlike the polar angle $\theta$, the rapidity and pseudorapidity separation, $\Delta y$ and $\Delta \eta$, are both invariant under Lorentz transformations.

As previously mentioned, the ATLAS detector is composed of several sub-detectors, each suited to a different purpose. Most of the sub-detectors have components which 
separately cover the barrel region within $0<|\eta|<1.5$, and the end-cap within $1.5<|\eta|<3.2$. The region between $3.2<|\eta|<4.5$ is considered the forward region, and contains its own specialized instrumentation. The innermost layer of ATLAS is the Inner Detector, which specializes in the measurement of the momentum and direction of charged particles. Enveloping the Inner Detector is the calorimeter system, comprised of the electromagnetic and hadronic calorimeters, with the former used to measure the energy deposition characteristics of electrons and photons, and the latter tailored towards the same measurements for all manner of hadrons. Along the edges of the ATLAS detector, a "forward calorimeter" system also exists to capture particles travelling at high rapidity. Lastly, the Muon Spectrometer is the outermost layer of ATLAS, and as the name implies, is tailored towards the measurement of the momentum, charge, and direction of muons, which in general interact only minimally with the other sub-detectors of ATLAS. The following sections will provide an overview of each component of ATLAS, with emphasis placed on those components which will be most relevant to later chapters of this work.

\subsubsection{Inner Detector}

The Inner Detector (ID) system in ATLAS encompasses several sub-detectors with different radial coverage, as shown in Figure 3.5. The nearest detector to the beam line is the Insertable B-Layer (IBL), which is surrounded by a silicon-based Pixel Detector. Enveloping the IBL and Pixel Detector is a Semiconductor Tracker (SCT) system, with the Transition Radiation Tracker (TRT) being the outermost layer. Both the Pixel and SCT detectors cover the region $|\eta|<2.5$, while the TRT covers the region $|\eta|<2$. The entire ID is immersed in a $2 \mathrm{~T}$ solenoidal magnetic field to 


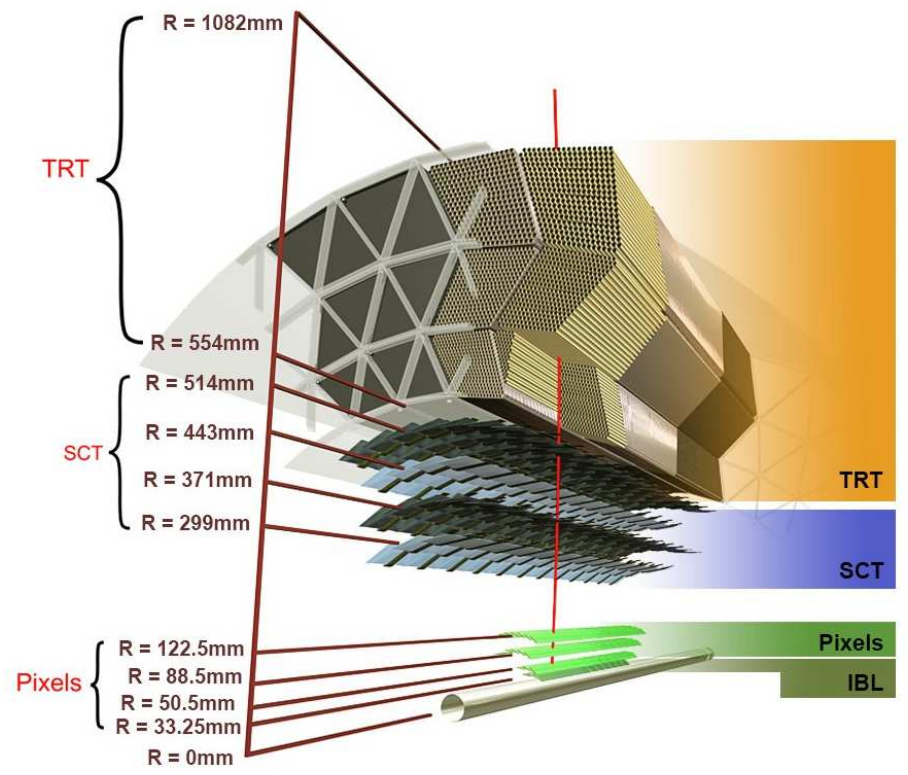

Figure 3.5: Sensors and structural elements of the Inner Detector, along with the trajectory of a charged track with $p_{\mathrm{T}}=10 \mathrm{GeV}$ in the barrel region $(\eta=0.3)$ [62].

allow for charged particle identification, as well as momentum and position measurement of tracks with momenta as low as $0.5 \mathrm{GeV}$. Tracks are essentially patterns of discrete depositions of energy which represent the trajectory of a charged particle as it traverses the Inner Detector. At small radii, the ID is capable of performing highresolution pattern recognition using discrete space points from the Pixel detector, in addition to stereo pairs of silicon microstrips from the SCT layer. At larger radii, the TRT weaves gaseous straw tube elements with transition radiation material to provide continuous tracking and electron identification over $|\eta|<2.0$.

\section{Insertable B-Layer}

The IBL is the innermost component of the ID, and provides the space-point measurements closest to the $p p$ interaction point, which are crucial for identifying tracks 
and vertices, as well as tagging potential $B$ mesons [63]. The latter function is particularly important for heavy meson identification in ATLAS, as $B$ mesons tend to cross this layer entirely before decaying, creating a distinct signature of one $B$-layer hit emerging into multiple tracks in subsequent ID layers. The IBL is structured as a barrel composed of 14 staves, where each stave is equipped with 16 or 32 modules. The main active component of each module is a pixel readout chip, which features an array of $80 \times 336$ pixels, with each pixel having a size of $50 \times 250 \mu \mathrm{m}^{2}$ [63]. The IBL was installed during the Phase I upgrade of the LHC, in order to manage the higher luminosity conditions during Run-II, as well as offset the performance degradation of the existing B-layer in ATLAS.

\section{Pixel Detector and Semiconductor Tracker}

The Pixel detector consists of three barrel layers and six disk layers, with three disks each in the forward and backward directions. The purpose of this detector layer is to provide high accuracy in track and vertex determination, yielding at least three space-point measurements per track. The detector is comprised of 1744 identical pixel sensors with nominal dimensions of $50 \times 400 \mu \mathrm{m}^{2}$, although the size extends to $50 \times 600 \mu \mathrm{m}^{2}$ in the regions at the front-end chips on a module. Each sensor hosts 47232 pixels, with each column of the front-end chip hosting four ganged pixels, totalling 46080 readout channels. The pixel modules are arranged in three barrel layers, along with two end-caps with three disk layers. The spatial resolution of individual pixel modules is quoted as $12 \mu \mathrm{m}$, with approximately $80 \%$ of tracks having at least one pixel hit [64].

The SCT consists of 4088 silicon-based strip modules which tile four coaxial, 
cylindrical layers in the barrel region, along with two end-caps containing nine disk layers each. The modules cover a total surface area of $63 \mathrm{~m}^{2}$, providing nearly hermetic coverage, which allows for four or more precision space-point measurements possible over the coverage of the ID. The 2122 barrel SCT modules are composed of $80 \mu \mathrm{m}$ pitch micro-strip sensors, providing a combined spatial resolution of $\sim 16 \mu \mathrm{m}$, as measured in the $R-\phi$ plane.

\section{Transition Radiation Tracker}

The basic elements of the TRT detector are polyimide straw tubes, each with a diameter of $4 \mathrm{~mm}$. Both the barrel and end-cap straws lead into anodes composed of $31 \mu \mathrm{m}$ diameter tungsten wires plated with $0.5-0.7 \mu \mathrm{m}$ gold. The anode wires of the barrel straws are read out at each end, with an active length of $\pm 71.2 \mathrm{~cm}$. The straw tubes themselves are filled with a $\mathrm{Xe} / \mathrm{CO}_{2} / \mathrm{O}_{2}$ gas mixture, with the straws operated in an envelope of $\mathrm{CO}_{2}$. For normal operating conditions, the maximum electron collection time is approximately $48 \mathrm{~ns}$, while the operational drift-time accuracy is $130 \mu \mathrm{m}$. The TRT records hits using straws interleaved with polypropylene, with up to 73 layers of straws in the barrel region, and 160 straw planes in the end-caps. In the barrel region, the TRT is divided into three rings of 32 modules each, while in the end-caps, the TRT is composed of two sets of independent wheels containing uniformly-spaced straws. A given charged track with $p_{\mathrm{T}}>0.5 \mathrm{GeV}$ and $|\eta|<2$ will cross at least 36 straws, or at least 22 straws in the region $0.8<|\eta|<1.0$ [64]. 


\subsubsection{Calorimeter system}

The calorimeter system in ATLAS contains multiple sampling detectors with full $\phi$ symmetry and coverage along the beam axis, as shown in Figure 3.6. Each calorimeter system is contained within a cryostat which allows the operating temperature of the calorimeter system to be suitably low to minimize noise and improve energy resolution. The active medium of each calorimeter is liquid argon, which was chosen for its linear behaviour, as well as the general stability and resistance of its response under continual radiation exposure. The calorimeter systems are arranged and divided roughly according to the types of particles they are best suited to measure. The barrel cryostat contains the electromagnetic barrel calorimeter, which is best suited to measurements of electrons and photons, as well as the tile calorimeter, which is tailored to measure hadronic radiation that travels more deeply into the detector. In the end-cap region, two cryostats contain the electromagnetic end-cap calorimeter (EMEC), as well as the hadronic end-cap calorimeter (HEC) behind it. In addition, a forward calorimeter (FCal) covers the region closest to the beam line.

Particles entering a calorimeter deposit energy by showering, inducing a cascade of electromagnetic and/or hadronic interactions within the active material of the detector. The depth at which the calorimeter can contain a shower is characterized by the type of interactions occurring. For EM showers, particles mainly lose energy through ionization and bremsstrahlung ("braking radiation"), and calorimeter depth is defined in terms of radiation lengths $\left(X_{0}\right)$, which is both the distance over which the mean energy loss of an electron to bremsstrahlung is all but $1 / e$, and for highenergy photons, $7 / 9$ of the mean free path before $e^{+} e^{-}$pair production [39]. For hadronic interactions, shower depth is described in terms of absorption lengths $(\lambda)$, 


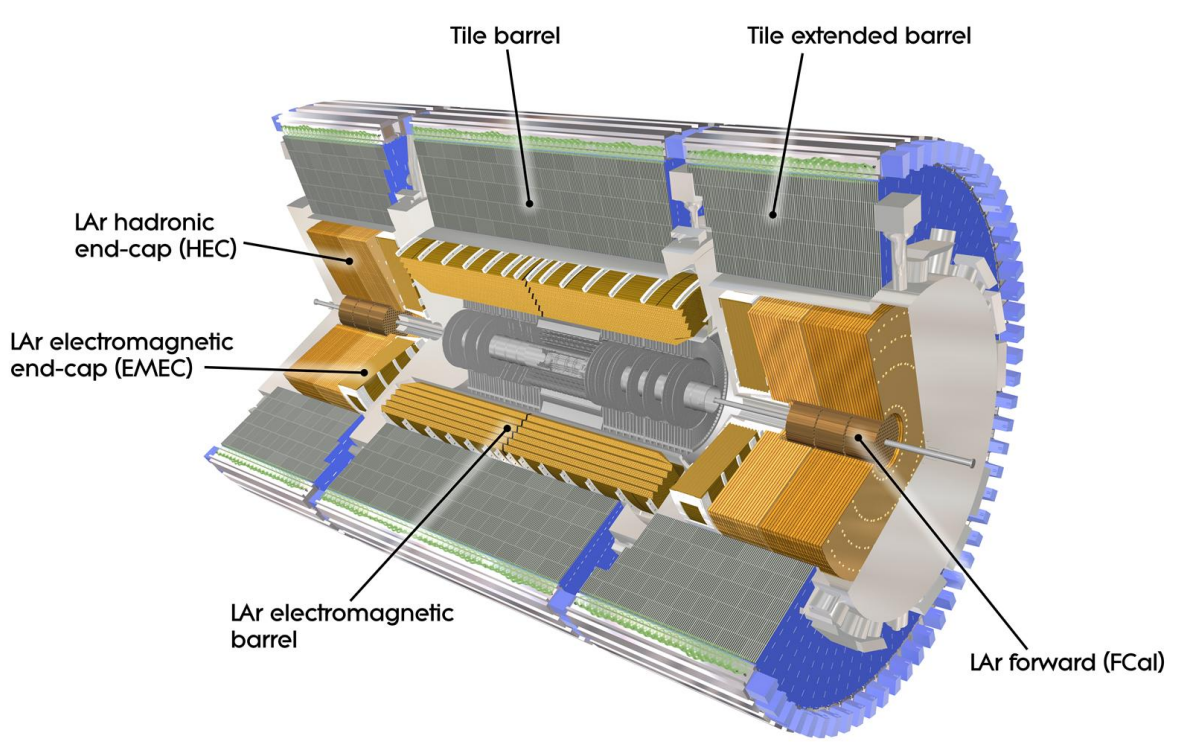

Figure 3.6: Cutaway view of the layers of the ATLAS calorimeter system [65].

which are characterized as the distance into a material in which a particle has lost $1 / e$ of its energy, but has not been absorbed [39]. If a particle shower extends beyond the active material depth of the calorimeter, only part of the energy of the particle will be measured.

\section{Electromagnetic calorimeter}

The electromagnetic calorimeter (ECAL) system contains lead-liquid argon detectors with accordion-shape absorbers and electrodes. The accordion geometry of the barrel and endcap calorimeters is chosen due to its ability to maintain a full coverage in $\phi$ without any cracks. Additionally, the uniformity in shape allows for consistent performance with regards to linearity and resolution as a function of $\phi$. The calorimeters span multiple active layers in depth; the precision-measurement region in $0<|\eta|<2.5$ spans three layers, while the higher- $|\eta|$ region in $2.5<|\eta|<3.2$ 
spans two layers, with an additional two layers found in the overlap region between the barrel and EMEC. The detector coverage is mostly hermetic in the $\eta$-direction up to the edge of detector acceptance, with the exception of a "crack" between the barrel and end-cap regions of the calorimeter system between $1.37<|\eta|<1.52$, which features only limited instrumentation [64].

The barrel region of the electromagnetic calorimeter is actually composed of two half-barrels which are centred about the $z$-axis. In particular, one half-barrel covers the region $0<\eta<1.475$, while the other covers $-1.475<\eta<0$. A single half-barrel is composed of 1024 accordion-shaped absorbers, with readout electrodes interleaved between the absorbers. A given LAr module has a thickness of $22 X_{0}$, although the thickness increases from 22 to $30 X_{0}$ between $|\eta|=0$ and 0.8 , and from 24 to $33 X_{0}$ between $|\eta|=0.8$ and 1.3 [64].

The different layers of the barrel ECAL are segmented to varying degrees, as shown in Figure 3.7. The first layer is segmented in strips along $\eta$, while the second and third layers are segmented both in $\eta$ and $\phi$. The front layer is roughly $4.4 X_{0}$ thick, with a strip size of $\Delta \eta=0.0031$, the high granularity of which aides in the identification of $\pi^{0} \rightarrow \gamma \gamma$ decays. The highest overall cell granularity is found in the second layer, with an individual cell size of $\Delta \eta \times \Delta \phi=0.025 \times 0.0245$. Lastly, the third layer constitutes a series of "trigger towers" with size $\Delta \eta \times \Delta \phi=0.1 \times 0.0982$, wherein individual cells have granularity $\Delta \eta \times \Delta \phi=0.05 \times 0.0245$. The purpose of these towers is to allow the data acquisition system to highlight a given recorded collision as potentially physically meaningful, as explained further in Section 3.2.4.

On each side of the electromagnetic barrel calorimeter, the EMEC calorimeters can be found. These calorimeters take the form of two wheels, which themselves consist 


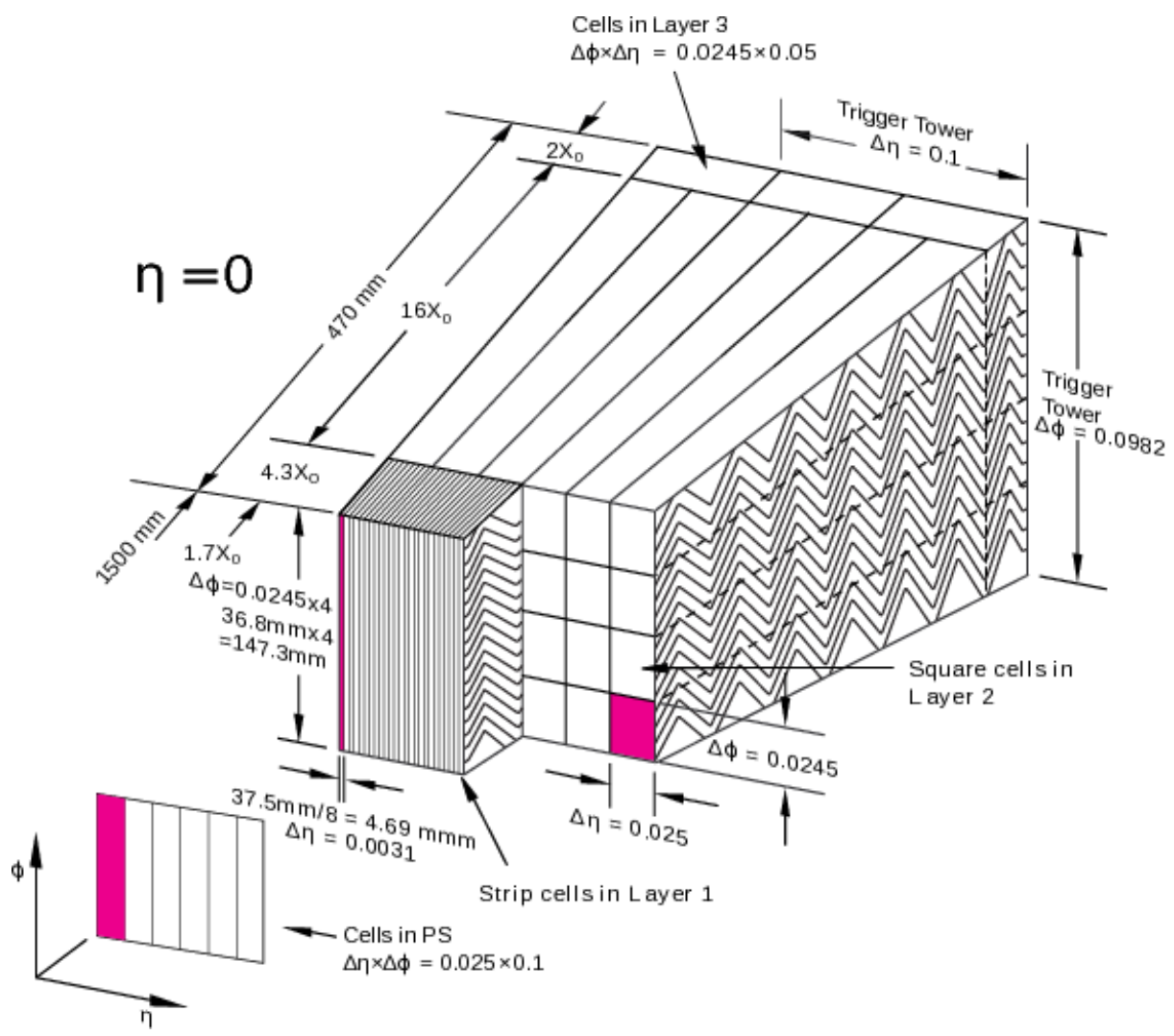

Figure 3.7: Sketch of the segmentation of a barrel module in the ECAL, showing the different layers and ganging of electrodes in the $\phi$-direction. The cell granularity in $\Delta \eta \times \Delta \phi$ is shown for each layer, along with the trigger towers [55]. 
of two co-axial wheels. The EMEC covers the region $1.375<|\eta|<3.2$, and each endcap has a total active thickness of at least $24 X_{0}$, except for the region $|\eta|>1.475$. The thickness increases from 24 to $38 X_{0}$ between $|\eta|=1.475$ and 2.5, and from 26 to $36 X_{0}$ between $|\eta|=2.5$ and 3.2. Within each end-cap, 768 absorbers are interleaved with readout electrodes in the outer wheel, while 256 absorbers are found in the inner wheel. Worth noting is that the granularity of the first layer is variable, with the granularity changing in $|\eta|$ in order to keep a consistent strip width. The transverse size of the projective cell in the middle layer is $\Delta \eta \times \Delta \phi=0.025 \times 0.025$, the same as that of the barrel region. The third layer has an equivalent granularity in $\Delta \phi$, but a twice coarser granularity in $\Delta \eta[64]$. Altogether, the target energy resolution for the ECAL system is [55],

$$
\frac{\sigma_{E}}{E}=\frac{10 \%}{E} \oplus \frac{17 \%}{E} \oplus 0.7 \%,
$$

where $E$ has units of $\mathrm{GeV}$.

The region $0<|\eta|<1.8$ also hosts a presampler in front of the electromagnetic calorimeter, which is a separate liquid-argon layer $11 \mathrm{~mm}$ in depth, made of 64 identical azimuthal sectors (or 32 / half-barrel). End-cap presamplers can be found in front of the end-cap calorimeters, as well, which consist of 32 identical, azimuthal modules, each with a granularity of $\Delta \eta \times \Delta \phi=0.025 \times 0.1$ [55]. The purpose of the presampler is to provide a measurement of the energy lost in front of the ECAL, which can be particularly important for the measurement of low energy electrons and photons, and of particles travelling within the crack region of $1.37<|\eta|<1.52$. 


\section{Hadronic calorimeters}

The hadronic calorimeter (HCAL) system in ATLAS can be broadly described in terms of three main subsystems: the tile calorimeter; the liquid-argon HEC; and the liquid-argon FCal. The tile calorimeter is a sampling calorimeter which uses steel as an absorber, and liquid-argon as the active medium. The tile calorimeter lies behind the ECAL, and is subdivided into a central barrel which covers the region $|\eta|<1.0$, and two extended barrels within $0.8<|\eta|<1.7$. Each barrel consists of 64 modules made of steel plates and scintillating tiles, with a longitudinal size $\Delta \phi \sim 0.1$. The depth of the tile calorimeter extends to roughly $7.4 \lambda$. Within the gap region between the barrel and extended barrel, a series of special steel-scintillator modules and thin scintillator counters are integrated which have the same sampling fraction as the rest of the tile calorimeter. Overall, the tile calorimeter has a measured energy resolution of $\sigma_{E} / E=56 \% \cdot \sqrt{\mathrm{GeV}} / \sqrt{E} \oplus 6 \%[55]$.

The HEC is a copper/liquid-argon sampling calorimeter, and covers the range $1.5<|\eta|<3.2$, and the modules share the same croystat as the EMEC and the FCal. The HEC is composed of two wheels in each end-cap cryostat: a front wheel (HEC1); and a rear wheel (HEC2). Each wheel is cylindrical in design, with an outer radius of $2030 \mathrm{~mm}$, and contains two longitudinal sections, constructed of 32 wedgeshaped modules. The FCal modules are located in the same cryostats as the end-cap calorimeters, and extend coverage over the region $3.1<|\eta|<4.9$. Each FCal module is split into three components: An electromagnetic module which covers roughly $2.7 \lambda$, and two hadronic modules which cover roughly 3.7 $\lambda$. The hadronic energy resolution of the $\mathrm{FCal}$ is $\sigma_{E} / E=70 \% \cdot \sqrt{\mathrm{GeV}} / \sqrt{E} \oplus 3 \%$ [55]. 


\subsubsection{Muon Spectrometer}

The Muon Spectrometer (MS) is the outermost part of the ATLAS detector, covering a region $|\eta|<2.7$. Its primary purpose is to detect and measure the momentum of charged particles exiting the barrel and end-cap calorimeters, primarily muons. Within the region of $|\eta|<2.4$, the MS is also capable of triggering data acquisition based on the presence of these particles, as discussed further in Section 3.2.4. The spectrometer is capable of measuring a large range in muon momenta, with good momentum resolution and charge identification possible from $\sim 3 \mathrm{GeV}$ up to $3 \mathrm{TeV}$. Like the Inner Detector, the Muon Spectrometer is immersed in a toroidal magnetic field, with the field strength of $\langle B\rangle=0.4 \mathrm{~T}$ allowing for a transverse momentum resolution of approximately $10 \%$ for tracks which have been reconstructed solely in the MS, with a momentum of $1 \mathrm{TeV}$ [55].

The precision-tracking chambers in the barrel are located on and between the coils of the superconducting toroid magnet, while in the end-cap, the chambers are in front and behind the two end-cap toroid magnets. The barrel chambers are arranged in three concentric, cylindrical shells around the beam axis, while the muon chambers form large wheels in the two end-cap regions, placed perpendicular to the $z$-axis. Worth noting is that a gap in coverage exists at $|\eta| \approx 0$, which is necessary to allow for access and repairs to the magnet, calorimeter, and tracking systems.

Precision momentum measurements are performed by the Monitored Drift Tube (MDT) chambers, consisting of three to eight layers of drift tubes. These chambers cover the pseudorapidity range $|\eta|<2.7$, with the exception of the innermost endcap layer, where coverage extends to $|\eta|<2.0$. In the region within $2<|\eta|<2.7$, the innermost tracking layer houses Cathode-Strip Chambers (CSC), which are multi- 


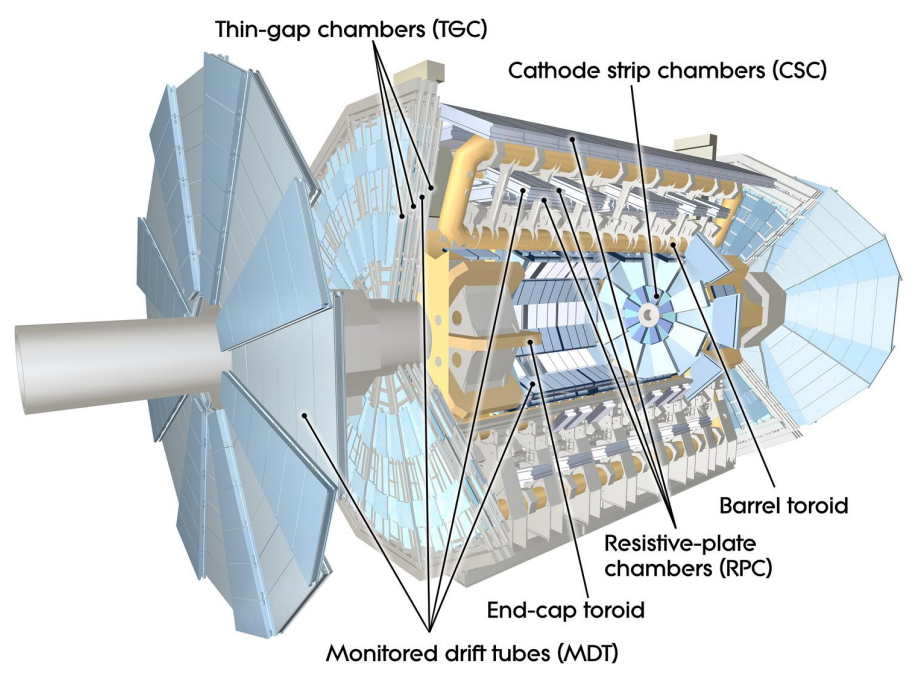

Figure 3.8: Cutaway view of the of the ATLAS Muon Spectrometer subsystem [55].

wire proportional chambers with cathode planes segmented into orthogonal strips. The purpose of the orthogonal segmentation is to allow for both coordinates to be measured from the induced-charge distribution. The resolution of a chamber is approximately $40 \mu \mathrm{m}$ in the bending plane, and $5 \mathrm{~mm}$ in the transverse plane [55].

Beyond the measurement systems in the MS, the Resistive Plate Chambers (RPC) and Thin Gap Chambers (TGC) cover regions of $|\eta|<1.05$ and $1.05<|\eta|<2.7$, respectively, and are used to provide triggering information for measurements containing muons. The RPC and TGC also serve to measure muon coordinates in a direction orthogonal to that of the MDT and CSC chambers. A high-precision optical alignment system is also implemented, which monitors the positions and internal deformations of the MDT chambers. Through use of the alignment system, along with track-based alignment algorithms, the locations of MDT wires and CSC strips along a muon trajectory can be known to better than $30 \mu \mathrm{m}$. 


\subsubsection{Trigger system}

The design luminosity of the LHC is $10^{34} \mathrm{~cm}^{-2} \mathrm{~s}^{-1}$, corresponding to a $40 \mathrm{MHz}$ bunch crossing rate, with an average of 25 proton-proton interactions per bunch crossing. With such a high multiplicity of instantaneous interactions, the trigger system was designed to reduce the input rate of data to a more manageable output rate of $\sim 200$ $\mathrm{Hz}$ for recording and offline processing [66]. The trigger system in ATLAS encompasses three "levels" with different purposes: The Level 1 (L1) trigger, which is a hardware-based system used to trigger detector readout; the Level 2 (L2) trigger, which applies preliminary event selection based on custom algorithms; and the event filter (EF) trigger, which looks at several types of reconstructed objects to isolate potentially meaningful events [67]. The latter two trigger levels (L2 and EF) together form the High Level Trigger (HLT).

The L1 trigger system is composed of custom-built fast electronics, and is designed to reduce the output rate to a maximum of $75 \mathrm{kHz}$. Prior to a decision by the $\mathrm{L} 1$ trigger, detector signals are stored in front-end pipelines, and information from the muon spectrometer and calorimeter systems lets the L1 trigger decide whether to accept the signals, and allow readout to the L2 trigger. When an event is accepted by the L1 trigger, data from the tracking detectors and calorimeter systems are transferred to detector-specific readout buffers used to store the event in fragments, pending the decision by the L2 trigger. Selection by the L2 trigger is based on the processing of detector information from several Regions of Interest (RoI) provided by the L1 trigger. The L2 trigger system further reduces the event rate to $\sim 3 \mathrm{kHz}$.

For events accepted by the L2 trigger, all event fragments from the readout buffers are built using specialized reconstruction software, thereby providing full event infor- 
mation to the EF. The EF is based mainly on offline algorithms which are invoked from custom interfaces for running in the trigger system. A combination of information on the quality of reconstructed objects, as well as preliminary object selection (e.g. energy or momentum cuts), is used to select events at a rate of $\sim 200 \mathrm{~Hz}$ with an average processing time of $\sim 4 \mathrm{~s} /$ event. Events which pass the EF trigger are written to disk at the target rate of $400 \mathrm{~Hz}$ [66]. 


\section{Chapter 4}

\section{Electron, photon reconstruction}

\section{using superclusters}

The reconstruction of electrons and photons - known within ATLAS as $e / \gamma$ reconstruction - is of critical importance in the study of many physical processes in ATLAS. Perhaps most notably, $e / \gamma$ objects are used to reconstruct the final states of the $H \rightarrow \gamma \gamma$ and $H \rightarrow Z Z^{*} \rightarrow 4 \ell$ processes, which compose the so-called "silver" and "gold" Higgs boson decay channels, respectively. Therefore, any changes to the $e / \gamma$ reconstruction which improve the efficacy of electron and photon measurements can potentially impact not just Standard Model measurements and Higgs physics, but future BSM searches, as well.

This chapter details a new $e / \gamma$ reconstruction method that not only reconstructs electrons and photons, but also searches and accounts for radiative losses in the Inner Detector by charged particles due to bremsstrahlung and other material interactions, providing overall better estimates of reconstructed particle energies. A particular focus will be placed on the derivation and performance of the electron reconstruction, 
which was primarily the work of the author. An overview of the existing $e / \gamma$ reconstruction framework is given, as well as a detailed discussion of the new algorithm. Validation studies for electrons and photons are shown before and after energy calibration, as well as studies for robustness against varying levels of pile-up. Lastly, results are shown detailing the impact on several analyses currently performed in ATLAS which use electrons.

\subsection{Overview of electron, photon reconstruction}

To provide a fair comparison of the performance of the previous and new $e / \gamma$ reconstruction methods, it is necessary to describe how each algorithm builds electrons and photons. In general, $e / \gamma$ reconstruction starts with two fundamental components: clusters and tracks. Clusters represent the energy deposited in the calorimeter by an electromagnetic shower. Both electrons and photons can leave electromagnetic showers in the ECAL, as they both interact with the liquid argon material in the calorimeter. Tracks, on the other hand, are uniquely formed by charged particles in the Inner Detector - namely, electrons and positrons, as well as $e^{+} e^{-}$pairs from photons that undergo pair production. Tracks can be formed from depositions in one or more of the sub-detectors of the ID, although most physics analyses in ATLAS tend to require a given track to have several hits in the Pixel and/or SCT detectors.

\subsubsection{Clustering}

In previous iterations of the $e / \gamma$ reconstruction, the sliding window algorithm was used to build clusters, which is based on the summation of calorimeter cells within a 


\begin{tabular}{lcc}
\hline \hline Particle type & Barrel size & End-cap size \\
\hline Electron & $3 \times 7$ & $5 \times 5$ \\
Converted photon & $3 \times 7$ & $5 \times 5$ \\
Unconverted photon & $3 \times 5$ & $5 \times 5$ \\
\hline \hline
\end{tabular}

Table 4.1: Cluster size in $n_{\eta} \times n_{\phi}$ cells for different particle types, in the barrel and end-cap regions of the ECAL.

fixed-size rectangular window. The position of the fixed-size window "slides" across the cell grid of the calorimeter until it contains a local maximum of transverse energy. The algorithm generally proceeds in three steps: tower building; precluster (seed) finding; and cluster filling [68]. In the first step, calorimeter towers are formed by collecting cells within all longitudinal layers in a region $\Delta \eta \times \Delta \phi$, and are used as an intermediate object for the subsequent seed finding.

In the seed finding step, a fixed-size window is moved across each element of the grid of calorimeter towers. If the transverse energy within this window (defined as the sum of the transverse energy of the towers contained in the window) is a local maximum, and is above a threshold energy, a pre-cluster is formed. If two pre-clusters sufficiently overlap in $\eta \times \phi$, only the pre-cluster with the largest transverse energy is kept. To build the final cluster, all ECAL cells within a fixed-size window around the pre-cluster barycentre are kept and summed together, where the barycentre is defined as the cluster position in the second ECAL layer, $\left(\eta_{\mathrm{L} 2}, \phi_{\mathrm{L} 2}\right)$. The size of the window varies depending on particle type and detector region, with the different options summarized in Table 4.1. The fixed sizes of $3 \times 7(5 \times 5)$ cells for electron clusters were optimized specifically for electrons to take into account the different overall energy distributions in the barrel (end-cap) calorimeters. 


\subsubsection{Tracking, loose track-cluster matching, and refitting}

Track reconstruction for electrons and converted photons proceeds in two steps: pattern recognition and track fitting. Generally speaking, a track seed consists of three hits, which can be in different layers of the silicon subsystems of the Inner Detector. The standard pattern recognition [69] takes the initial track seeds and applies a pion hypothesis to model energy loss within detector material, while also extending its trajectory through the length of the Inner Detector to create a track candidate. If a track seed with $p_{\mathrm{T}}>1 \mathrm{GeV}$ and seven or more hits cannot be successfully extended to a full track using the pion hypothesis, and it overlaps spatially with an EM cluster, the pattern recognition algorithm is retried with an electron hypothesis, using a method built around the Kalman filter-smoother formalism [70]. This approach is more "flexible" than the pion hypothesis, allowing for kinematic changes due to $\leq 30 \%$ energy loss at each material interaction point, which accounts for radiative energy losses due to bremsstrahlung. All track candidates are then fitted using the ATLAS Global $\chi^{2}$ Track Fitter [69], which can perform fits using either an electron or pion hypothesis. The electron and pion hypotheses proceed identically, with the exception that the electron hypothesis compensates for bremsstrahlung losses in the $\chi^{2}$ calculation. A track candidate can be refitted using the electron hypothesis if it fails the pion hypothesis track fit due to a large $\chi^{2}$ (e.g. due to large energy loss).

After the collection of fitted tracks is built, a loose matching is performed between EM clusters and tracks to identify potential electron and converted photon candidates [71]. For a given track, an extrapolation is performed from the perigee (or point of closest approach to the beam line) to the second layer of the ECAL, and a track-cluster match is formed if the distance in $\Delta \eta$ and $\Delta \phi$ is sufficiently small be- 
tween the extrapolated track end-point, and the cluster barycentre. Tracks with less than four silicon hits, or TRT-only tracks, are extrapolated from the last measurement point. A track-cluster match can also occur if a track overlaps a cluster within a narrower window when its momentum is rescaled to match the cluster energy. In the latter case, the momentum rescaling procedure allows for the retention of tracks whose measured momenta do not adequately match their associated calorimeter energies, such as in cases of radiative energy loss due to bremsstrahlung.

Once a set of track-cluster matches has been defined, tracks with silicon hits have their parameters precisely re-estimated using the Gaussian Sum Filter (GSF) algorithm, which implements a non-linear generalization of the Kalman filter algorithm [70]. This improvement in precision is achieved by accounting for non-linear effects from radiative energy losses in the ID, particularly in the transverse plane. The result from the Global $\chi^{2}$ Track Fit is kept for TRT-only tracks, along with the $\mathcal{O}(0.01 \%)$ of tracks which fail to be refitted by the GSF [71].

\subsubsection{Electron candidate reconstruction}

The previous method of reconstructing electron candidates is described in detail in Ref. [71], while a brief description follows here for context. An electron candidate is reconstructed if at least one track is matched to a given cluster. The track-cluster matching proceeds as described in Section 4.1.2, but using GSF-refitted tracks and tighter requirements on the separation in the $\phi$-direction. Worth noting is that, in general, more than one track can be associated to a given cluster.

The best-matched track, both in terms of measurement quality and overlap with the cluster, is chosen as the primary track, thereby determining the kinematics and 
charge of the electron. In general, tracks are required to have at least one hit in the Pixel detector; this requirement reduces the number of random matches between nearby tracks, which may occur due to bremsstrahlung interactions, for example. If more than one associated track has pixel hits, the tracks are sorted according to several criteria [71]:

- The distance between the cluster barycentre and the extrapolated track in the second layer of the ECAL

- The cluster barycentre and extrapolated track distance, with the track momentum scaled to correspond to the cluster energy

- The number of pixel hits for a given track, with a stronger preference given for tracks with earlier hits in the Pixel detector

Each electron candidate cluster is rebuilt in a sequential manner across the three ECAL layers and the presampler, starting from the second layer, using the window sizes defined in Table 4.1. The cluster position is corrected in each layer to account for the distribution of the deposited energy.

The reconstructed electron candidates are also subject to an identification routine which is based on tracking and calorimeter information [71]. The purpose of this identification scheme is to distinguish true electrons emerging from the HS interaction from those emerging from photon pair production, or "fake" electrons that are the result of e.g. hadrons from pile-up interactions. The identification works by way of a multivariate likelihood discriminant. In particular, the discriminant is trained using simulated "true" and "fake" electrons, encompassing several relevant variables: the energy leakage into the HCAL; the fraction of energy deposited in the third ECAL 
layer; shower width variables; and track-cluster matching variables. The signal and background probabilities for a given electron candidate are combined together into a single discriminant of the form,

$$
d_{\mathcal{L}}=\frac{\mathcal{L}_{S}}{\mathcal{L}_{S}+\mathcal{L}_{B}}, \quad \mathcal{L}_{S(B)}(\vec{x})=\prod_{i=1}^{n} P_{S(B), i}\left(x_{i}\right),
$$

such that physics analyses may then apply thresholds on the likelihood-based discriminant to achieve target selection efficiencies for true electrons.

\subsubsection{Photon candidate reconstruction}

The previous method of reconstructing unconverted and converted photon candidates is described in detail in Ref. [72]. The use of tracking together with clustering primarily affects the reconstruction of single- and double-track converted photons, for which the trajectory of one or both particles in the $\gamma \rightarrow e^{+} e^{-}$pair is recorded in the Inner Detector, respectively. A series of geometric requirements on the distance and angle between pairs of adjacent tracks are imposed to ensure that they emerge parallel at the so-called conversion vertex [72]. These geometric requirements serve as an input to a $\chi^{2}$-based fit to constrain the conversion vertex, which is used to estimate the distance from the beam line to the vertex in the transverse plane. To be retained for later use, a conversion vertex must have sufficiently small $\chi^{2}$ from the constrained fit, and the conversion radius of the vertex must be sufficiently large.

The reconstruction of asymmetric (or single-track) conversions can occur when one of the produced electron tracks is too soft (below $p_{\mathrm{T}}<0.5 \mathrm{GeV}$ ), or because the two tracks are very close to each other and cannot be adequately separated. In such cases, tracks without hits in the B-layer that either have an electron identification 
discriminant value greater than $95 \%$, or have no hits in the TRT, are considered as "single-track" conversion vertex candidates. For single-track conversion candidates, the conversion vertex is defined as the location of the first measurement of the track. Tracks which pass through a passive region of the B-layer are not considered as singletrack conversions unless they are missing a hit in the second Pixel layer.

In order to match conversion vertices to clusters, the conversion candidates are extrapolated to the second layer of the ECAL, with the extrapolated $(\eta, \phi)$ compared to those of the cluster barycentre. In the case of single-track conversion vertex candidates, the extrapolation is peformed using the track, starting from its last measurement point. For double conversions with spatially close tracks, both tracks are extrapolated to the calorimeter, while double conversions with more widely spaced tracks extrapolate the direction of the photon from the fitted conversion vertex. In order to be considered matched to a cluster, the extrapolation is required to pass tight cuts on $\Delta \eta$ and $\Delta \phi$, similar to the track-cluster matching of electrons. Any EM clusters which remain unmatched to tracks or conversion vertices are considered unconverted photon candidates.

\subsubsection{Ambiguity resolution}

Since conversion tracks may go unmatched to a vertex, and vertices may be made of spurious converted photon tracks, there may be some remaining ambiguity as to whether a reconstructed object should be classified as an unconverted photon, converted photon, or electron. Therefore, an ambiguity resolution routine is used to try to distinguish the particle type for a given $e / \gamma$ object [71, 72].

In general, clusters which are unmatched to any track or vertex are considered 
unconverted photon candidates, while clusters matched to a conversion vertex candidate are considered converted photon candidates. If a converted photon and electron are both reconstructed from the same cluster, the electron track is compared to the $\operatorname{track}(\mathrm{s})$ matched to the conversion vertex candidate. If the track is found to be part of the conversion vertex, then the converted photon candidate is retained, with the exception of cases where one track has a B-layer hit, and the other does not. Conversely, if the electron candidate track does not coincide with any track in the conversion vertex, and the track is sufficiently high $p_{\mathrm{T}}$, then the conversion vertex is removed. If the track of an electron candidate has no silicon detector hits, the object is reconfigured as a single-track converted photon candidate if $p_{\mathrm{T}}^{\text {track }}<2 \mathrm{GeV}$, and $E_{\text {clus }} / p_{\text {track }}<10$.

\subsection{Motivation for superclusters}

The primary focus of the new $e / \gamma$ reconstruction is the move to dynamic, variablesize clusters, which offers numerous advantages over the previous, fixed-size clusterbased approach. Historically, the main reason for not using variable-size clusters was the difficulty in deriving an energy calibration, but more advanced, multivariate calibration techniques [73] have freed the reconstruction from this constraint. With these changes, the possibility emerges to use topological clusters (or topo-clusters) [74] as a foundation to reconstruct EM showers, as described in Section 4.3. The growth of topo-clusters is comparable to the natural growth of an EM shower in a calorimeter - namely, within the Molière radius [75] - leading to dynamically-sized clusters that can grow across several calorimeter layers. Furthermore, topo-clusters generally maintain a high energy resolution across a wide range of deposited energies. 


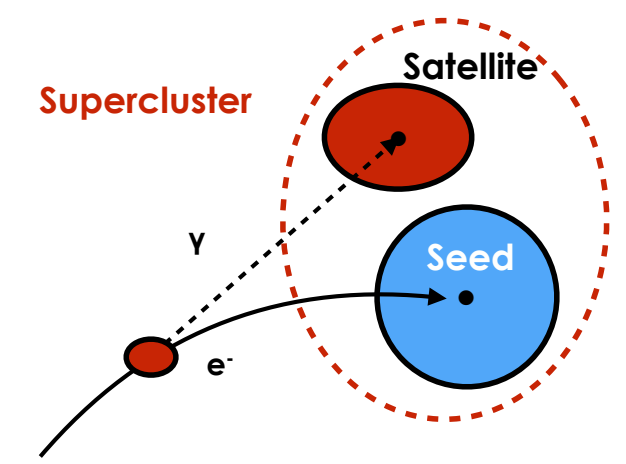

Figure 4.1: Diagram of an example supercluster showing a seed electron cluster and a satellite photon cluster.

Topo-clusters are also unique in their ability to reconstruct EM showers down to the $\mathcal{O}(100 \mathrm{MeV})$ range in energy. Therefore, a topo-cluster-based reconstruction may be used to recover low energy photons radiated due to bremsstrahlung interactions in the Inner Detector, and connect them to their associated electron or converted photon, forming what may be termed superclusters. An example diagram of a supercluster is given in Figure 4.1, in which an electron may radiate a bremsstrahlung photon due to material interactions with the Inner Detector.

In practical terms, the supercluster algorithm attempts to associate the seed cluster formed from the primary electron shower with nearby satellite clusters, which may originate from photons emitted due to bremsstrahlung, or from regions of the electron shower which were reconstructed as separate topo-clusters. By combining seed and satellite clusters together, one can recover some or all of the energy lost due to bremsstrahlung. As a result, a significant increase in energy resolution is possible, due to bringing the reconstructed energy of the combined clusters nearer to the true energy deposited by the EM shower. Similarly, for photons that convert to electronpositron pairs, superclusters can include more of the energy of the original photon, 
since photon conversions can produce multiple topo-clusters, which can be merged into one supercluster.

\subsection{Topo-cluster reconstruction}

In contrast to the sliding window algorithm, topo-clusters are formed in a way that more closely follows the patterns of energy deposition created by particle showers. In particular, the observable which governs the seeding and growth of a topo-cluster is the cell significance, $\varsigma_{\text {cell }}^{\mathrm{EM}}$, which is computed as [68],

$$
\varsigma_{\text {cell }}^{\mathrm{EM}}=\left|\frac{E_{\text {cell }}^{\mathrm{EM}}}{\sigma_{\text {noise,cell }}^{\mathrm{EM}}}\right|,
$$

where $\left|E_{\text {cell }}^{\mathrm{EM}}\right|$ is the absolute cell energy at the EM scale and $\sigma_{\text {noise,cell }}^{\mathrm{EM}}$ is the expected cell noise. The standard topo-cluster reconstruction algorithm in ATLAS begins by forming proto-clusters which are seeded from calorimeter cells with $\varsigma_{\text {cell }}^{\mathrm{EM}} \geq 4$. The initial cell then collects nearby cells with significance $\varsigma_{\text {cell }}^{\mathrm{EM}} \geq 2$. Each neighbour cell passing the $2 \sigma$ noise threshold then becomes a seed cell in the next iteration, collecting each of its neighbours in the proto-cluster. Two proto-clusters can also be merged if they contain the same cell with $2 \sigma$ significance. After all nearby cells with $2 \sigma$ significance have been collected, a final layer of single, neighbouring cells with $\varsigma_{\text {cell }}^{\mathrm{EM}} \geq 0$ are added to the cluster. The topo-clusters built using this set of noise thresholds are commonly known as "4-2-0" topo-clusters.

After the initial collection of proto-clusters is formed, any proto-clusters with two or more local maxima can be split into separate clusters. A local maximum is defined by a cell energy of $E_{\text {cell }}^{\mathrm{EM}}>500 \mathrm{MeV}$, in addition to requirements that the maximum 
cell has at least four neighbours, and that none of the neighbours have a larger signal. Cells can also be shared between two proto-clusters which have split into separate topo-clusters, with the cell assigned a fractional weight for each cluster based on the energies of the clusters, and the distances of the cell to the centre of gravity of the two clusters $\left(d_{1}, d_{2}\right)$,

$$
w_{\text {cell }, 1}^{\text {geo }}=\frac{E_{\text {clus }, 1}^{\mathrm{EM}}}{E_{\text {clus }, 1}^{\mathrm{EM}}+r E_{\mathrm{clus}, 2}^{\mathrm{EM}}}
$$

where $r=\exp \left(d_{1}-d_{2}\right)$, and $d_{i}$ are expressed in units of the typical EM shower size scale in the ATLAS calorimeters. The weight for the cell in the second split cluster is given by,

$$
w_{\mathrm{cell}, 2}^{\mathrm{geo}}=1-w_{\mathrm{cell}, 1}^{\mathrm{geo}} \text {. }
$$

\subsection{Supercluster electron, photon reconstruction}

The following sections provide a detailed description of how clusters built from the existing topo-clustering method are adapted for use in electron and photon reconstruction, as well as how seed and satellite cluster candidates are grouped to form superclusters. As with the previous $e / \gamma$ reconstruction, an electron is defined as an object consisting of a cluster and a matched track, a converted photon is a cluster matched to a conversion vertex, and an unconverted photon is a cluster matched to neither a track, nor a conversion vertex. A diagram of the algorithm flow for the new electron and photon reconstruction is given in Figure 4.2. In the first stage of the reconstruction, EM topo-clusters are built from existing topo-clusters for later use in 
building electrons and photons, as described in Section 4.4.1. As with the previous algorithm, a GSF-based refitting of tracks is performed, with tracks subsequently matched to clusters. The algorithm also builds conversion vertices out of the refitted tracks and matches them to clusters.

In the new, second stage of the reconstruction, electron and photon supercluster algorithms are run independently, as described in Section 4.4.2. After applying preliminary corrections to the resultant superclusters, the reconstruction algorithm recomputes the distance between tracks matched to the electron superclusters, and conversion vertices matched to the photon superclusters. Since one object may be reconstructed as both an electron and a photon, an ambiguity resolution algorithm is performed to remove part of the overlap. However, some overlap is still allowed in order to maintain a high reconstruction efficiency for electrons and photons, to which physics analysis groups may apply their own criteria. The final electrons and photons are then built and calibrated, as described in Section 4.6.

\subsubsection{Building EM topoclusters}

The standard ATLAS topo-cluster reconstruction is not limited to one region of the detector, as cells from both the ECAL and HCAL may be included in a single topocluster. Therefore, the new $e / \gamma$ reconstruction algorithm starts by copying the existing 4-2-0 topo-clusters, and removing the hadronic calorimeter cells from each cluster. This new set of clusters contains cells only from the ECAL, as well as the presampler, and the E4 scintillators located within $1.4<|\eta|<1.6$. After removing the hadronic calorimeter cells, the cluster position and kinematics must be recalculated using the remaining cells, as the barycentre, uncalibrated energy, and other properties of the 


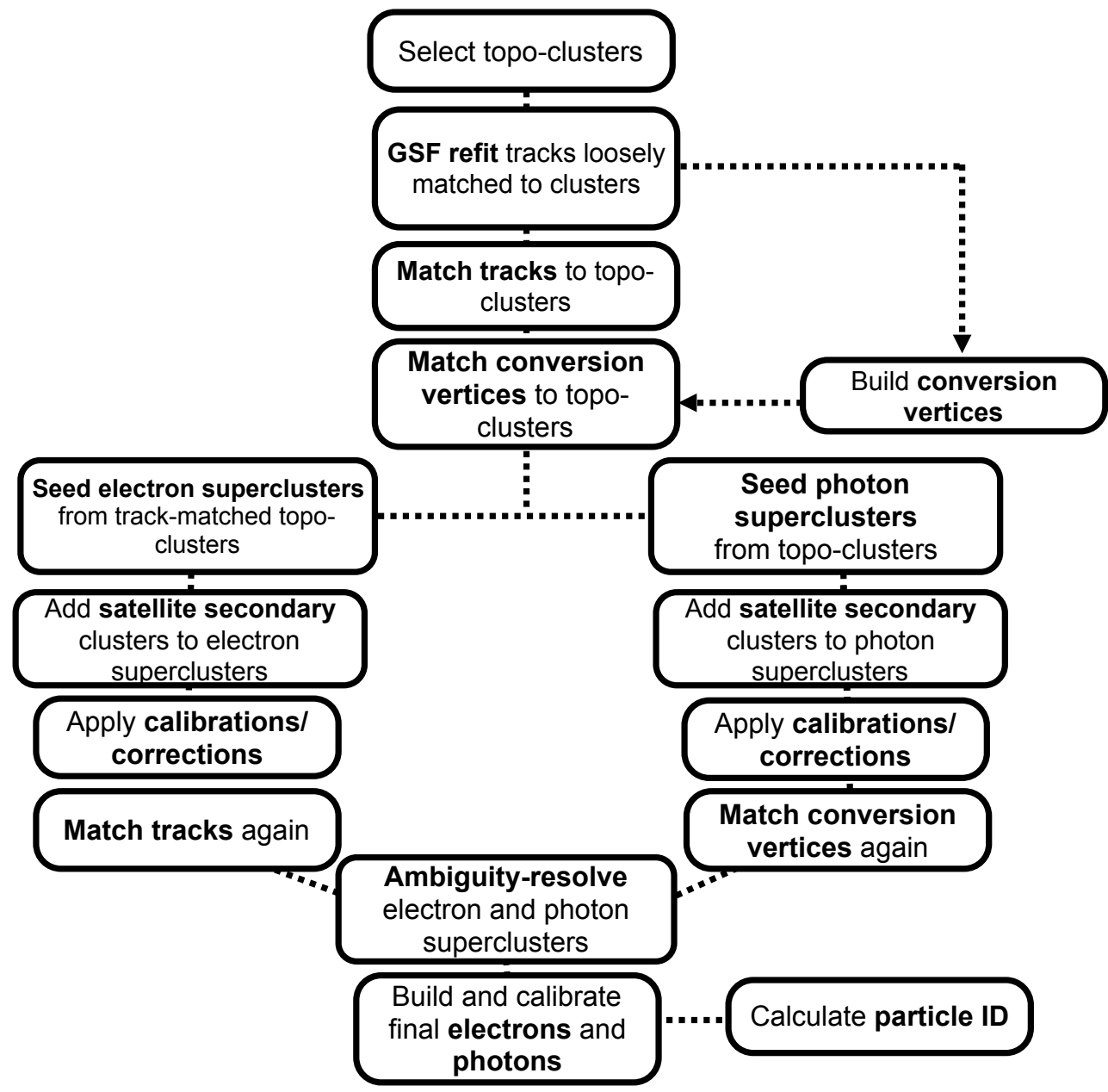

Figure 4.2: Algorithm flow diagram for the new electron and photon reconstruction. 
cluster may have changed in the process.

One important consideration when using topo-clusters is the robustness of the reconstruction algorithm against the selection of spurious EM clusters originating from pile-up interactions. To this end, for a topo-cluster to be accepted for use in the reconstruction, it must have more than $400 \mathrm{MeV}$ of energy deposited in the ECAL. This threshold was chosen to be similar to that which was previously set for topocluster-based studies of $J / \psi \rightarrow e^{+} e^{-}$decays [76], as well as studies of $\pi^{0} \rightarrow \gamma \gamma$ decays at $\sqrt{s}=900 \mathrm{GeV}[77]$. The threshold also roughly corresponds to the momentum threshold required for charged particles to traverse the Inner Detector and reach the EM calorimeter.

An initial selection cut is also made on the EM fraction of each topo-cluster, $f_{\mathrm{EM}}$, defined as,

$$
f_{\mathrm{EM}}=\frac{E_{\mathrm{L} 1}+E_{\mathrm{L} 2}+E_{\mathrm{L} 3}+w \cdot\left(E_{\mathrm{E} 4}+E_{\mathrm{PS}}\right)}{E_{\text {clus }}}, \quad w= \begin{cases}1, & 1.37<|\eta|<1.63 \\ 0, & \text { otherwise }\end{cases}
$$

where $E_{\mathrm{L} 1 / 2 / 3}$ are the energies deposited in layers 1,2 , and 3 of the ECAL, respectively. The term $\left(E_{\mathrm{E} 4}+E_{\mathrm{PS}}\right)$ is the sum of the energies deposited in the E4 scintillators and presampler, and is only considered for clusters within the region of $1.37<|\eta|<1.63$, as electrons and photons in this $\eta$ region tend deposit a non-trivial amount of energy in the presampler and E4 scintillators. The term is excluded in other detector regions, as the majority of the EM energy deposition tends to occur in the ECAL.

In principle, the optimal value of $f_{\mathrm{EM}}$ should be that which limits the initial acceptance of pile-up clusters, while maximizing the efficiency of selecting primary 


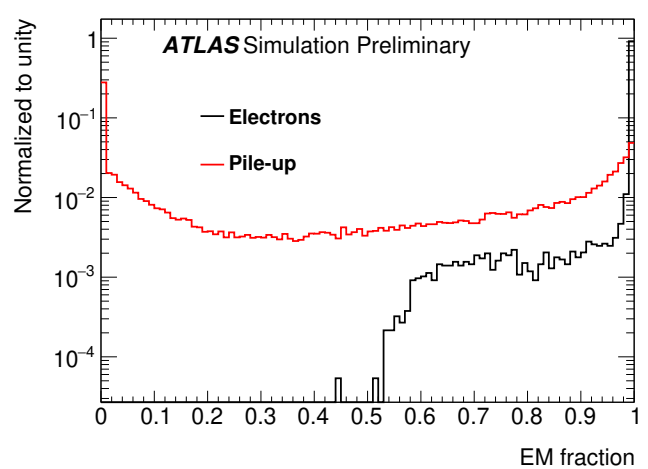

(a) $f_{\mathrm{EM}}$

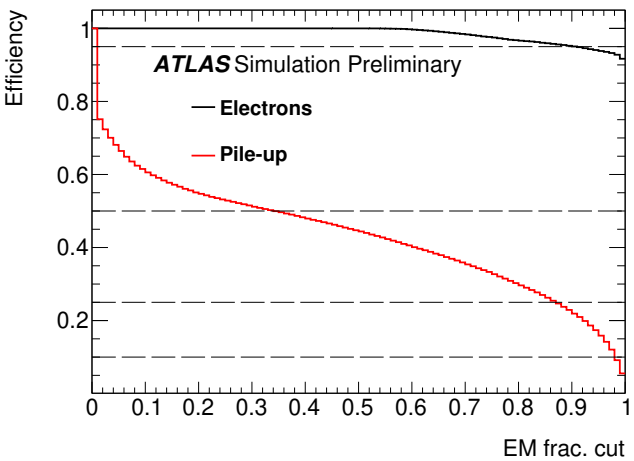

(b) Efficiency

Figure 4.3: Distributions of $f_{\mathrm{EM}}$ and reconstruction efficiency for true electron clusters (black) and pile-up (red).

electron clusters. To determine this optimal threshold, efficiency studies were performed using samples of simulated single electrons produced using the GEANT-based PARTICLE Gun software [78, 79], and passed through a full simulation of the ATLAS detector. The simulated samples were generated under two conditions: without simulated pile-up effects; and with a pile-up level of $\langle\mu\rangle \sim 25-30$. In the latter scenario, any clusters within $|\Delta \eta|<0.2$ and $|\Delta \phi|<0.2$ of the generated electron in each event were removed to obtain a sample containing clusters solely originating from pile-up interactions. Figure 4.3 shows the resultant distributions of $f_{\mathrm{EM}}$, along with plots of the reconstruction efficiency for various cuts on $f_{\mathrm{EM}}$, considering both true electron clusters and pile-up clusters. Ultimately, $f_{\mathrm{EM}}>0.5$ was chosen as a pre-selection requirement on the initial topo-clusters, as it rejected $\sim 60 \%$ of pile-up clusters without limiting the efficiency for selecting true electron topo-clusters. 


\subsubsection{Defining and building superclusters}

The reconstruction of an electron and photon supercluster proceeds in two stages: Firstly, a given topo-cluster is evaluated for use as a seed cluster candidate, which forms the basis of the supercluster; and secondly, in order to recover topo-clusters which have emerged from bremsstrahlung radiation or other material interactions, clusters in close proximity to the seed candidate are evaluated as satellite cluster candidates. The seed cluster selection stage begins with the list of EM topo-clusters defined in Section 4.4.1. The list of potential seed clusters is sorted according to descending transverse energy $\left(E_{\mathrm{T}}\right)$, defined as,

$$
E_{\mathrm{T}}=\sqrt{m^{2}+p_{\mathrm{T}}^{2}}
$$

where $p_{\mathrm{T}}$ is the transverse momentum,

$$
p_{\mathrm{T}}=|\vec{p}| / \cosh (\eta)
$$

such that higher $E_{\mathrm{T}}$ seed clusters collect only lower $E_{\mathrm{T}}$ satellite clusters. A given candidate electron seed cluster required to have a minimum $E_{\mathrm{T}}$ of $1 \mathrm{GeV}$. Furthermore, any potential seed cluster must be matched to a track with $\geq 4$ silicon (IBL, Pixel, SCT) hits. For photons, because no track match is required, the minimum $E_{\mathrm{T}}$ threshold for a cluster to form a seed candidate is raised to $1.5 \mathrm{GeV}$.

Once a cluster is found which meets the criteria to be a seed, the satellite finding stage begins, in which the remaining, lower $E_{\mathrm{T}}$ clusters must meet certain criteria to be considered satellite clusters. This process is summarized diagrammatically in Figure 4.5. If a given cluster is selected as a satellite, it is vetoed from future usage 

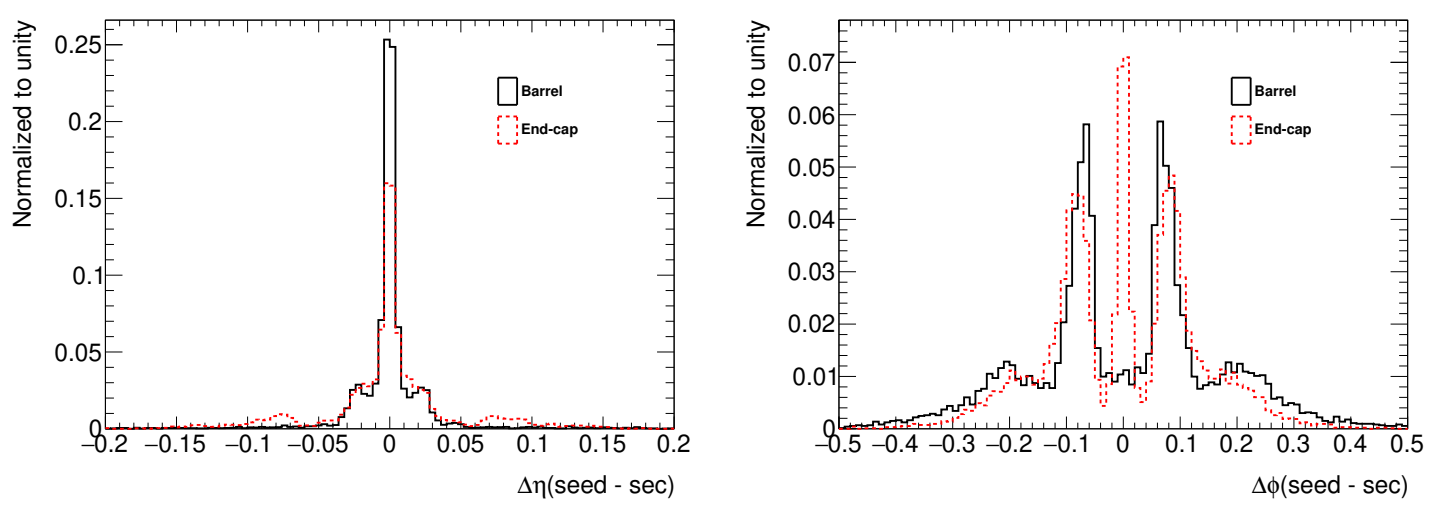

Figure 4.4: Distance in $\Delta \eta$ and $\Delta \phi$ between the barycentres of seed and satellite clusters in simulated single electron clusters.

as a seed cluster. Likewise, clusters which have been designated as seed candidates are vetoed for usage as satellite clusters. For both electrons and photons, a cluster is accepted as a satellite if it falls within a window of $\Delta \eta \times \Delta \phi=0.075 \times 0.125$ around the seed cluster barycentre, or equivalently,

$$
\left|\Delta \eta_{\text {seed-sat }}\right|<0.075 / 2 \text { and }\left|\Delta \phi_{\text {seed-sat }}\right|<0.125 / 2,
$$

as these cases tend to represent a significant population of secondary EM showers in both the barrel and end-cap regions of the EM calorimeter, as shown in Figure 4.4 for clusters reconstructed from simulated single electrons.

Another aspect of Figure 4.4 that is worth noting is the peaking in $\Delta \phi$ located roughly at $\Delta \phi= \pm 0.06$, which occurs because electron and positron tracks bend more strongly in the Inner Detector after bremsstrahlung, while the radiated photon will propagate in a straight line. Hence, there tends to be a displacement of a few cells in the $\phi$-direction between the barycentres of the primary and secondary EM showers. Due to the greater amount of detector material in the end-cap, an additional 


\section{All $e^{ \pm}, y:$}

Add all clusters within $3 \times 5$ window around seed cluster.

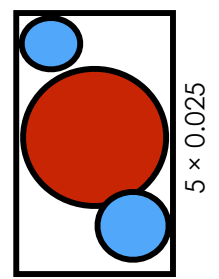

$3 \times 0.025$

\section{Electrons only:}

Seed, secondary cluster match the same track.

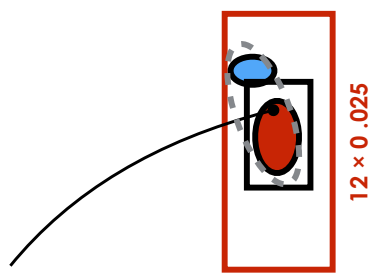

$5 \times 0.025$

\section{Converted photons only:} Add topo-clusters that have the same conversion
vertex matched as the seed cluster.

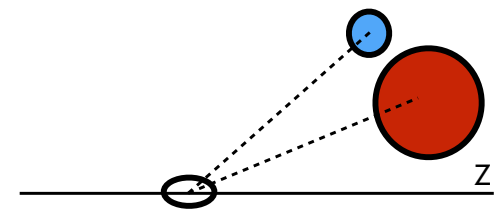

Add topo-clusters with a track match that is part of the conversion vertex matched to the seed cluster.

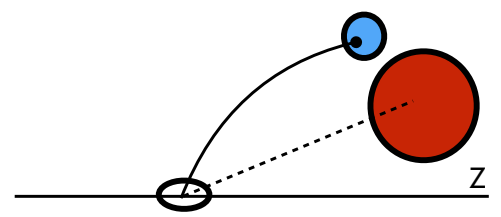

Figure 4.5: Steps of the superclustering algorithm for electrons and photons.

peak emerges at $\Delta \phi \sim 0$ composed of topo-clusters which have split from the cluster formed from the primary EM shower, as described in Section 4.3. These clusters tend to exist primarily in layers other than L2, where electrons deposit most of their energy, hence these clusters appear to "overlap" with the cluster formed from the primary EM shower.

For candidate electrons and converted photons, secondary satellite cluster searches are also performed to identify more distant clusters originating from early radiation in the Inner Detector, which may or may not convert into $e^{+} e^{-}$pairs. Tracking information is used in these searches to discriminate distant radiative photons from pile-up noise, or other unrelated clusters. For electrons, an additional satellite cluster search is performed using a larger window of $\Delta \eta \times \Delta \phi=0.125 \times 0.3$ around the cluster barycentre. Within this window, a satellite cluster can be associated to the 
seed cluster if it has $\geq 1$ matched tracks, such that the "best-matched" track for both clusters is the same.

This selection choice is motivated by the empirical observation that clusters built from showers of radiated photons tend to be matched to the same track as the cluster from the primary electron shower, as shown in Figure 4.6. In these plots, a comparison of cluster $\Delta \eta$ and $\Delta \phi$ for simulated single electrons is made under two scenarios: between primary electron clusters and actual secondary clusters; and between primary electron clusters, and non-primary EM clusters which are matched to the primary track. In the latter scenario, there is no explicit requirement that the non-primary EM clusters match actual satellite clusters from e.g. bremsstrahlung interactions, meaning that these distributions may include both actual secondary clusters and pile-up clusters which have been erroneously associated to the primary electron track.

It is clear that, within the larger window $\Delta \eta \times \Delta \phi=0.125 \times 0.3$ between clusters, there is a high concentration of actual secondary clusters matched to the primary electron track, evidenced by the good overlap in shape between the two distributions, particularly so for $\Delta \phi$. The notable absence of a peak around $\Delta \phi \sim 0$ in the endcap track-matched clusters is due to the fact that clusters used for track-matching are required to have a significant amount of energy deposited in L2. Therefore, clusters with energy deposited mainly in L1 or L3, such as those which split from the primary topo-cluster, will not be used for track-matching. In practice, however, they will be acquired as satellite clusters, as they fall within the initial $\Delta \eta \times \Delta \phi=$ $0.075 \times 0.125$ window around the seed cluster barycentre. Outside of the region $\Delta \eta \times \Delta \phi=0.125 \times 0.3$, spurious secondary clusters also become more prominent (pile-up, in actuality), particularly in the distribution of $\Delta \phi$, which does not taper 


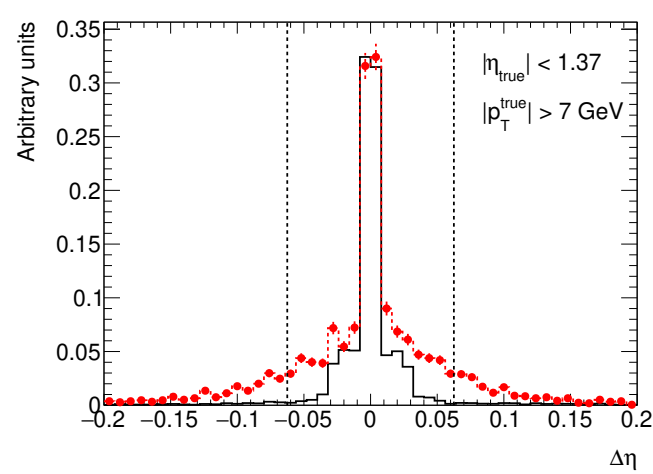

(a) Cluster $\Delta \eta$, barrel

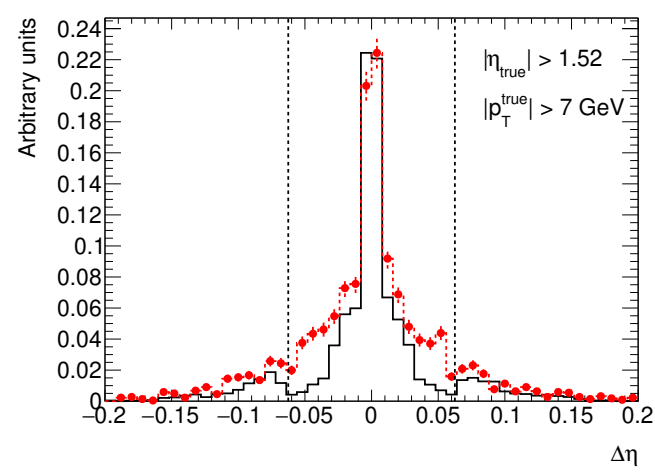

(c) Cluster $\Delta \eta$, end-cap

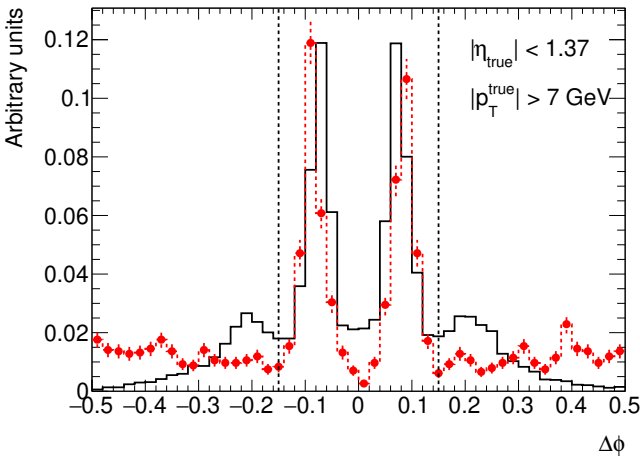

(b) Cluster $\Delta \phi$, barrel

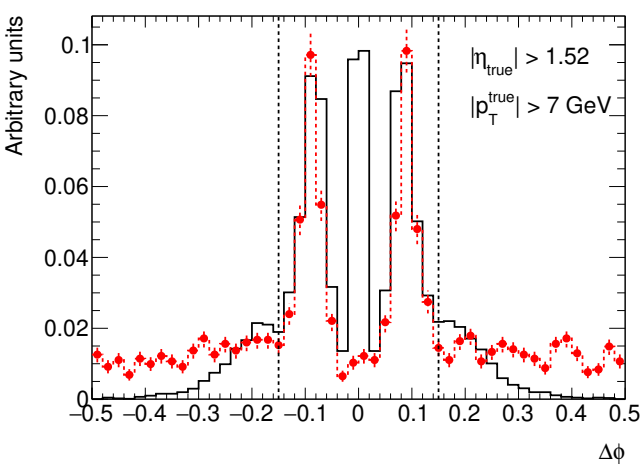

(d) Cluster $\Delta \phi$, end-cap

Figure 4.6: Distances in $\Delta \eta$ and $\Delta \phi$ between cluster barycentres under two scenarios: Between simulated primary electron topo-clusters and true secondary topo-clusters (black); and between simulated primary electron topo-clusters, and non-primary EM topo-clusters which are matched to the primary track (red). A window of $\Delta \eta \times \Delta \phi=0.125 \times 0.3$ is also demarcated on each plot.

off as rapidly as the $\Delta \phi$ plot made using true secondary clusters.

For converted photons, a cluster can also be designated a satellite candidate if it is either matched to a track that is one track of the conversion vertex associated to the seed cluster, or it has the same matched conversion vertex as the seed cluster. By default, only the "best-matched" tracks and conversion vertices are used for the matching. Furthermore, only tracks that contain silicon hits, and conversion vertices made up only of tracks containing silicon hits, are used for satellite cluster matching. 
Once a candidate seed cluster has been associated to a number of satellite candidates, the final step of the supercluster algorithm is to combine the cells of each individual cluster to make the final, combined cluster, known as cell summation. When combining topo-clusters, only cells from the first three ECAL layers are considered, as well as the presampler and the E4 scintillators in the crack region. Due to topo-cluster splitting, it is possible for two separate clusters to contain the same cell, with each cluster receiving a fraction of the cell energy. If two clusters which share cells are summed together, the energy of the summed clusters should equal the summed energies of the individual clusters, as each cell can only contribute a maximum of $E_{\text {cell }}$ to the overall construction.

Ignoring material interactions, which spread mainly in the $\phi$-direction, the development of an EM shower typically has a known lateral size of a few Molière radius units, such that $90 \%$ of the energy is contained in one unit, and $95 \%$ in two units [75]. Therefore, prior to cell summation, the size of each constituent topo-cluster in the supercluster is restricted to three (five) cells across in the $\eta$ direction in the barrel (end-cap) region, with respect to the cluster barycentre. This restriction is made by removing cells which fall outside of the threshold in $\eta$. Such a restriction limits the acquisition of "extraneous" cells which do not contain meaningful amounts of true energy, while also not limiting the growth of the cluster in the $\phi$-direction, where material interactions are most prevalent. An illustration of the effect of the $\eta$-restriction with an electron supercluster is shown in Figure 4.7, where the distribution of cells is shown in the second EM calorimeter layer, and the $z$-axis is the base-10 logarithm of the cell energy.

The effects of limiting the lateral topo-cluster size can also be observed in the 

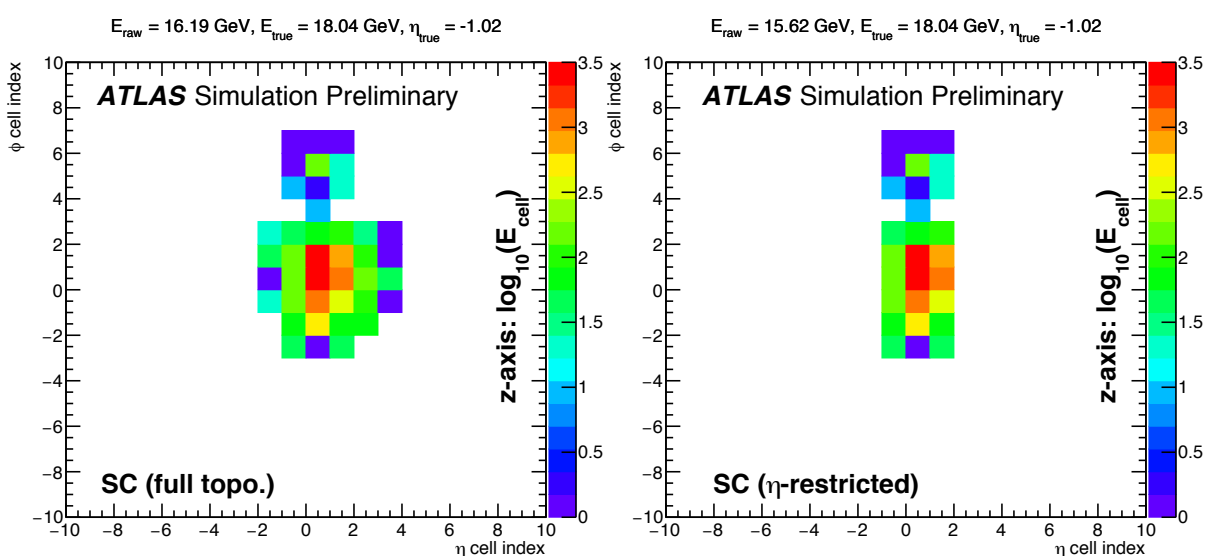

Figure 4.7: Effect of restricting cluster size in $|\eta|$ on a sample electron supercluster, in the second EM calorimeter layer. The $z$-axis scale is the base-10 logarithm of the cell energy.

shower containment $E_{\text {true }}^{\text {cluster }} / E_{\text {true }}^{\text {show }}$, or the ratio of the energy contained in the cluster, to the total energy deposited by the particle shower in the calorimeter. As shown in Figure 4.8, the average shower containment for $\eta$-restricted superclusters is either on-par with clusters built using the sliding window algorithm, or the change with increasing shower energy is smaller than electrons built using full topo-clusters. Similarly, Figure 4.9 shows that the average number of cells acquired per topo-cluster is more gradual with increasing shower energy for electrons built using $\eta$-restricted topo-clusters. The latter behaviour suggests a better degree of robustness against the acquisition of extra cells from e.g. pile-up interactions at higher electron energies, as it demonstrates the acquisition of true shower energy is in a sense decoupled from the lateral growth of the cluster after roughly two units of Molière radius.

\subsection{Uncalibrated supercluster performance}

Prior to performing any calibration or energy corrections, it must first be established that the new $e / \gamma$ reconstruction algorithm improves on the uncalibrated (or "raw") 

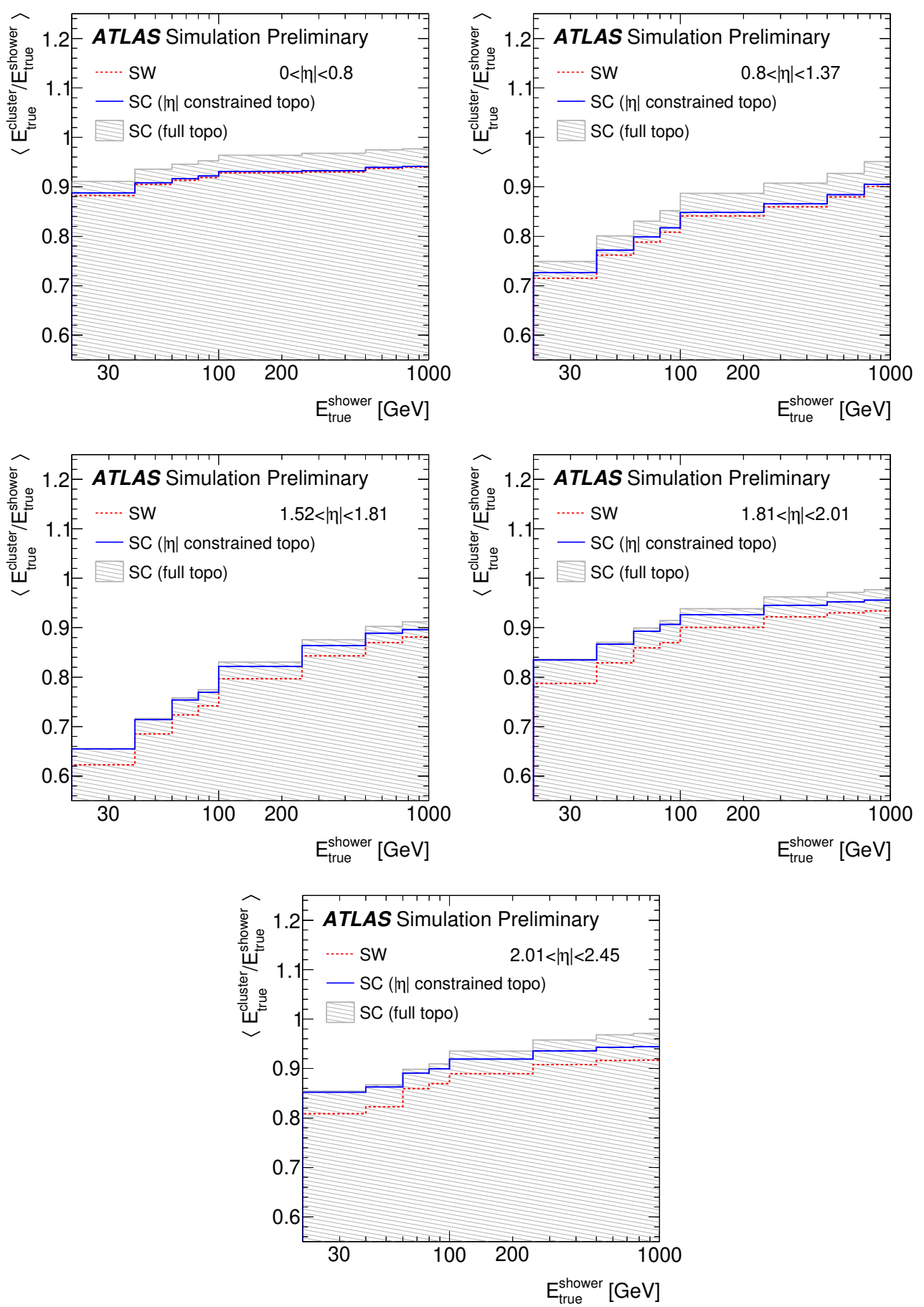

Figure 4.8: Average shower containment distributions for electrons reconstructed via sliding window and supercluster-based approaches. Included also are superclusters built using topo-clusters allowed unrestricted growth in $|\eta|$. 

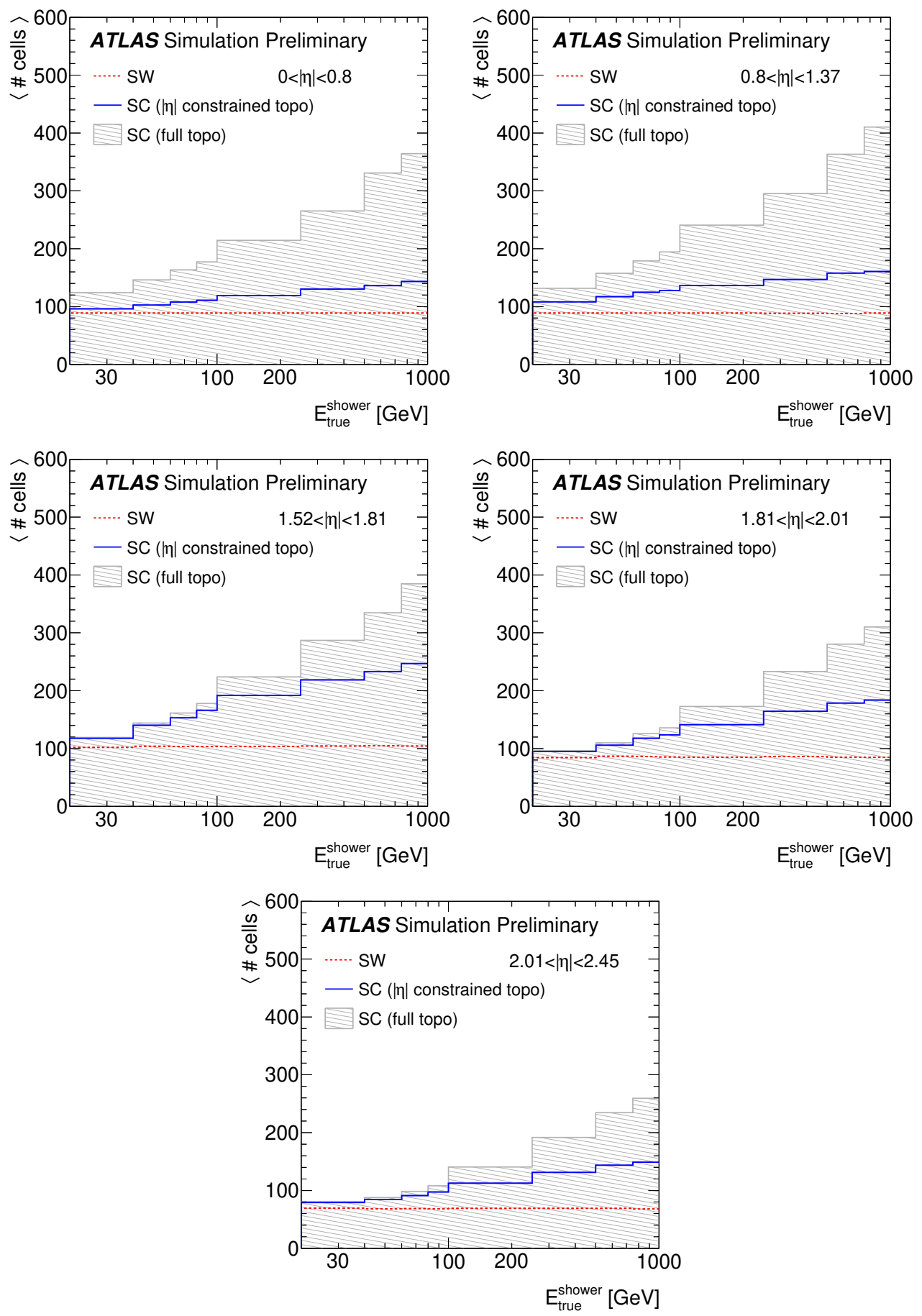

Figure 4.9: Distributions of the average number of cells contained in electron clusters reconstructed via sliding window and supercluster-based approaches. Included also are superclusters built using topo-clusters allowed unrestricted growth in $|\eta|$. 
energy response. In this context, the quantities of interest for assessing performance with respect to the previous reconstruction algorithm are the peak and width (resolution) of the energy response, $E_{\text {raw }} / E_{\text {true }}$, where $E_{\text {true }}$ is the true energy of the simulated particle, prior to any detector simulation, and $E_{\text {raw }}$ is the uncalibrated accordion energy, defined as $E_{\mathrm{raw}}=E_{\mathrm{L} 1}+E_{\mathrm{L} 2}+E_{\mathrm{L} 3}$.

To make a comparison between the behaviour of the previous and new $e / \gamma$ reconstruction algorithms, simulated samples of single electrons, converted photons, and unconverted photons were produced using the Geant-based Particle Gun software, and passed through a full simulation of the ATLAS detector. These single particles samples were then reconstructed separately using each reconstruction algorithm. The uncalibrated cluster energy serves as an input to the energy calibration, as described in Section 4.7. Therefore, an improvement in the uncalibrated energy response should translate to similar gains in the calibrated energy response, if the residual miscalibration of the new algorithm is equivalent (or smaller) to that of the previous algorithm.

In order to quantify the resolution of the energy response, the effective interquartile range (IQE) is used, defined as,

$$
\mathrm{IQE}=\frac{Q_{3}-Q_{1}}{2 \Phi^{-1}(0.75)} .
$$

In this equation, $Q_{3}-Q_{1}$ is the interquartile range (IQR) of the energy response, where $Q_{1}$ and $Q_{3}$ are the first and third quartiles of the distribution. As an example, the quartiles of a unit-width Normal distribution are shown in Figure 4.10, where the area between two quartiles encompasses $25 \%$ of the full distribution. Accordingly, the IQR is defined as the width of the region which contains the middle $50 \%$ of the 


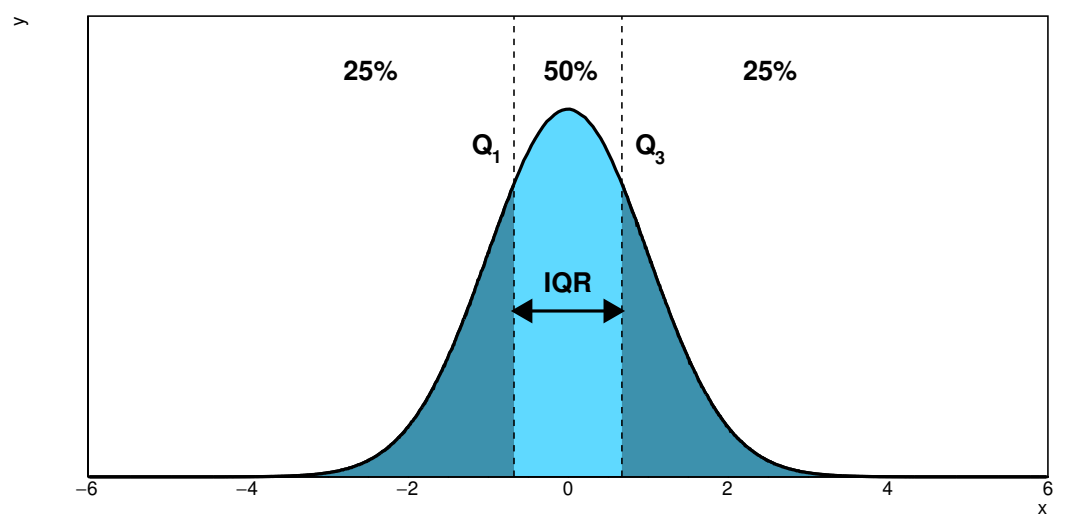

Figure 4.10: Diagram showing the IQR of a unit-width Normal distribution, where $Q_{1}$ and $Q_{3}$ are the first and third quartiles of the distribution.

distribution. The term $\Phi^{-1}(0.75)$ represents the inverse of the cumulative distribution function of the standard Normal distribution evaluated at 0.75 . In practice, resolution improvements should manifest in an IQE which is as small as possible; for example, an energy resolution of $\mathcal{O}(10 \%)$ would correspond roughly to an IQE of 0.1 , while a poor resolution of $\mathcal{O}(100 \%)$ would correspond to IQE $=1$.

For the purpose of characterizing energy resolution, the IQE tends to be used because it more properly factors in asymmetric behaviour in the tails of the energy response. In particular, long tails for the leftmost region of $E_{\text {raw }} / E_{\text {true }}$ can be prevalent for low energy electrons, where radiative energy losses can limit the amount of energy measured through reconstructing the electron cluster, as shown in Figure 4.11. Using the width of a Gaussian fit around the peak of the energy response, for example, may underestimate the impact of this important response region. The peak position of the energy response distribution is also used as a metric of performance, albeit in a secondary manner. In particular, a peak closer to $E_{\text {raw }} / E_{\text {true }}=1$ indicates that, on average, the reconstructed clusters capture more of the energy deposited by particles in the calorimeter. To obtain the peak of the response distribution, a Gaussian fit in 


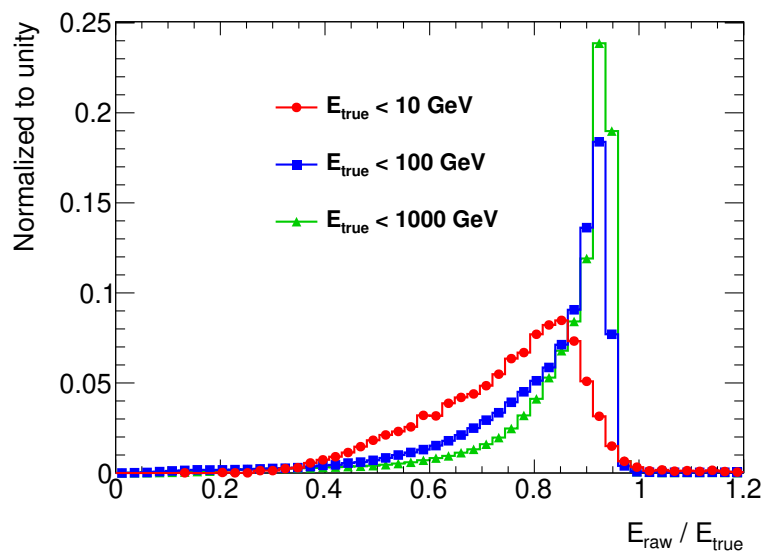

Figure 4.11: An example of the change in uncalibrated energy response for electrons in different truth energy regions, built using sliding window clusters.

a region $[-3 \sigma, 2 \sigma]$ is applied around the mean of the distribution, where the initial $\sigma$ is taken from RMS of the response distribution.

\subsubsection{Electrons, converted photons}

Comparisons of the uncalibrated response of simulated single electrons built using sliding window cluster and supercluster-based approaches are given in Figures 4.12 and 4.13. In particular, Figure 4.12 shows a comparison of the IQE of the two methods in different regions of $\left|\eta_{\text {true }}\right|$ and $E_{\mathrm{T}}^{\text {true }}$, while Figure 4.13 shows the fitted Gaussian peak for the same regions. The reconstructed electrons in these distributions are required to be true primary electrons, and pass loose electron quality requirements [80].

In most regions of phase space, the performance of the supercluster algorithm either matches that of the sliding window in terms of resolution and peak position, or improves upon it. A notable improvement in resolution can be seen for low $E_{\mathrm{T}}$ electrons in the central region of the detector, where the effects of bremsstrahlung interactions tend to be most prevalent. Notable resolution improvements are also 
found in the end-cap region of the detector where the amount of active material is larger, due to the ability of the topo-cluster to grow dynamically and absorb more deposited energy. At higher $E_{\mathrm{T}}$, the two algorithms tend to converge to the same resolution, although the fitted Gaussian peak is consistently closer to $E_{\text {raw }} / E_{\text {true }}=1$ for the supercluster method, suggesting that a topo-cluster-based reconstruction tends to capture more true energy than the sliding window-based approach.

Comparisons of the uncalibrated energy response of converted photons built using sliding window and supercluster-based approaches are given in Figure 4.14 and Figure 4.15, showing the IQE of the two methods in different regions of $\left|\eta_{\text {true }}\right|$ and $E_{\mathrm{T}}^{\text {true }}$, and fitted Gaussian peak for the same regions, respectively. The reconstructed converted photons in these distributions are required to be true primary photons, and pass loose photon quality requirements [81]. Similar to the electron performance, the supercluster algorithm for converted photons either matches the performance of the sliding window algorithm, or improves upon it. As with the reconstructed electrons, the largest resolution improvements can be found in the end-cap region of the detector, as well as in the central region for low $E_{\mathrm{T}}$ converted photons. The similarity of improvement can be attributed to the fact that converted photons are ultimately built from reconstructed electrons and positrons, making them subject to the same material interactions and bremsstrahlung effects.

\subsubsection{Unconverted photons}

Comparisons of the uncalibrated response of unconverted photons built using sliding window and supercluster-based approaches are given in Figures 4.16 and 4.17. In particular, Figure 4.16 shows a comparison of the IQE of the two methods in different 

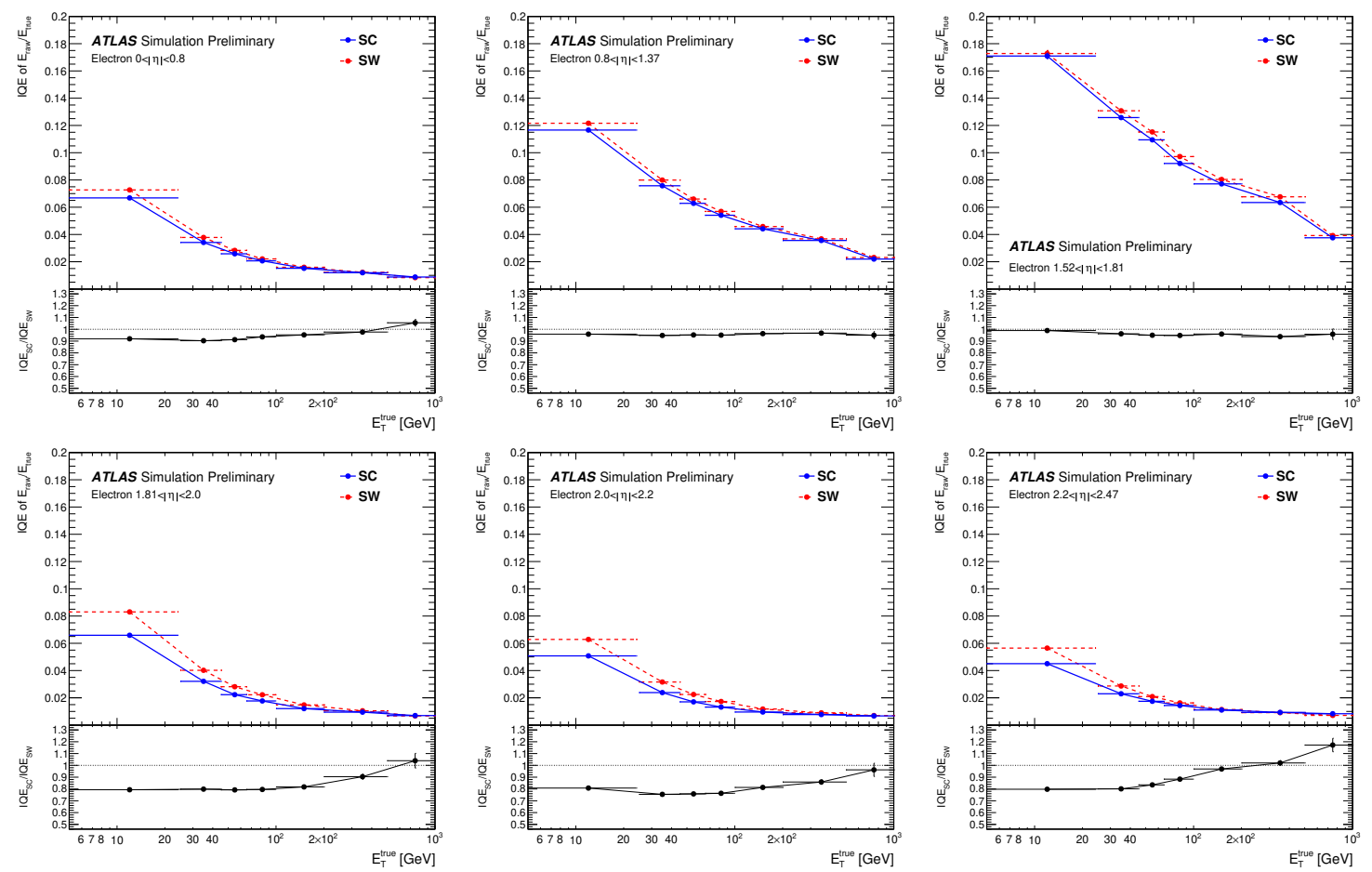

Figure 4.12: Comparison of uncalibrated IQE values between sliding window (red) and supercluster (blue) electrons, using samples of single electron events.
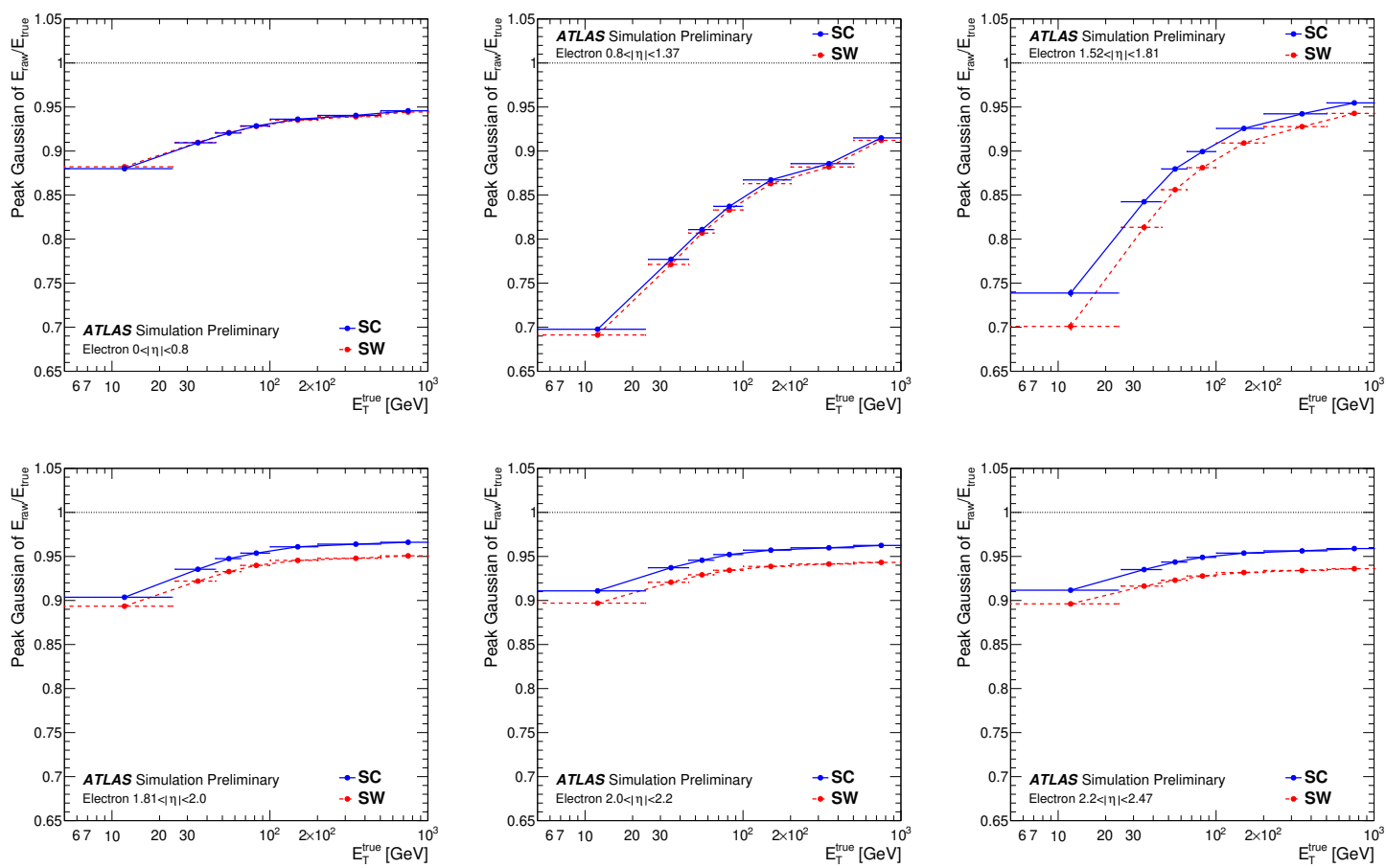

Figure 4.13: Comparison of uncalibrated Gaussian peak values between sliding window (red) and supercluster (blue) electrons, using samples of single electron events. 

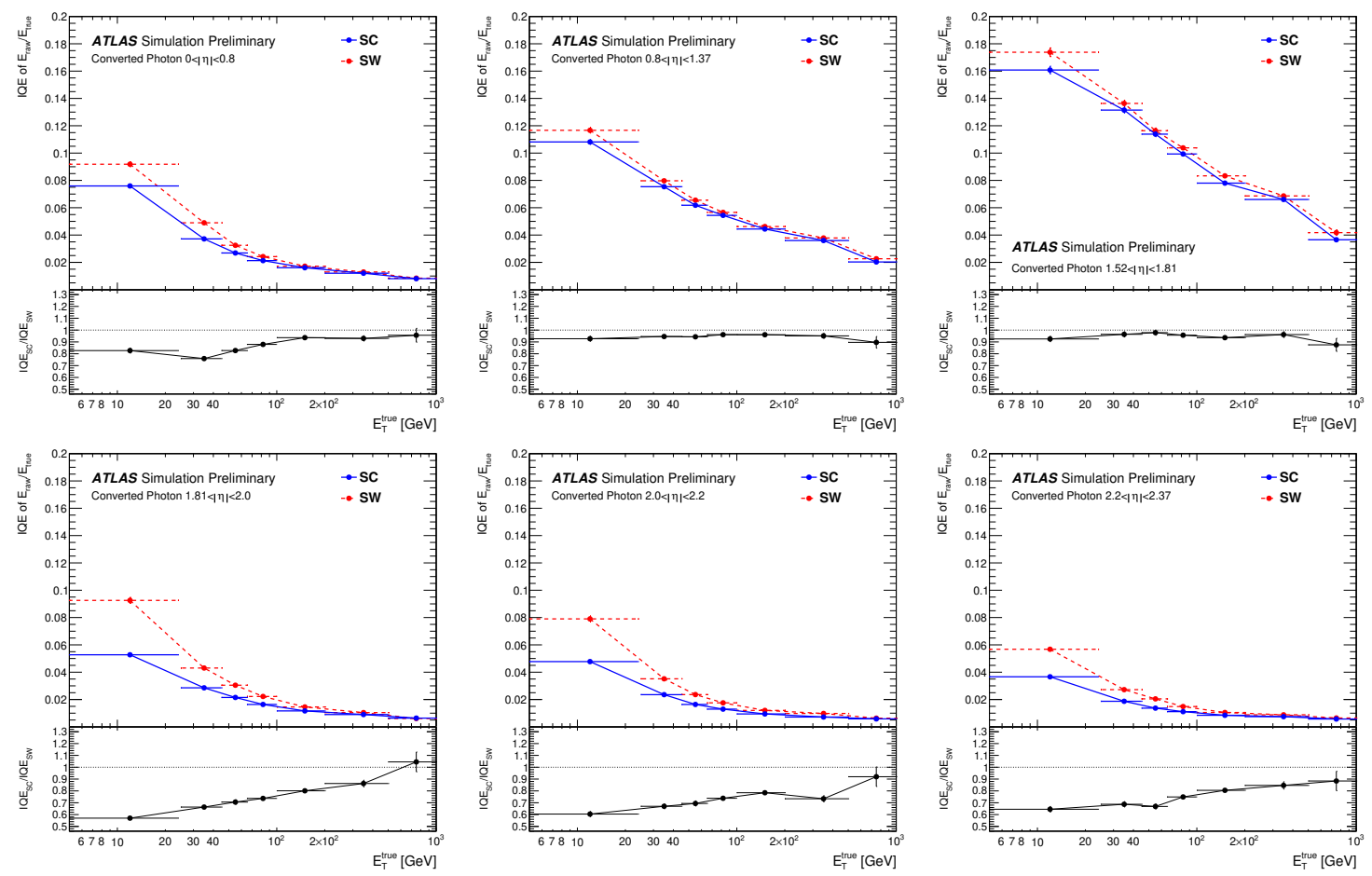

Figure 4.14: Comparison of uncalibrated IQE values between sliding window (red) and supercluster (blue) converted photons, using samples of single converted photon events.
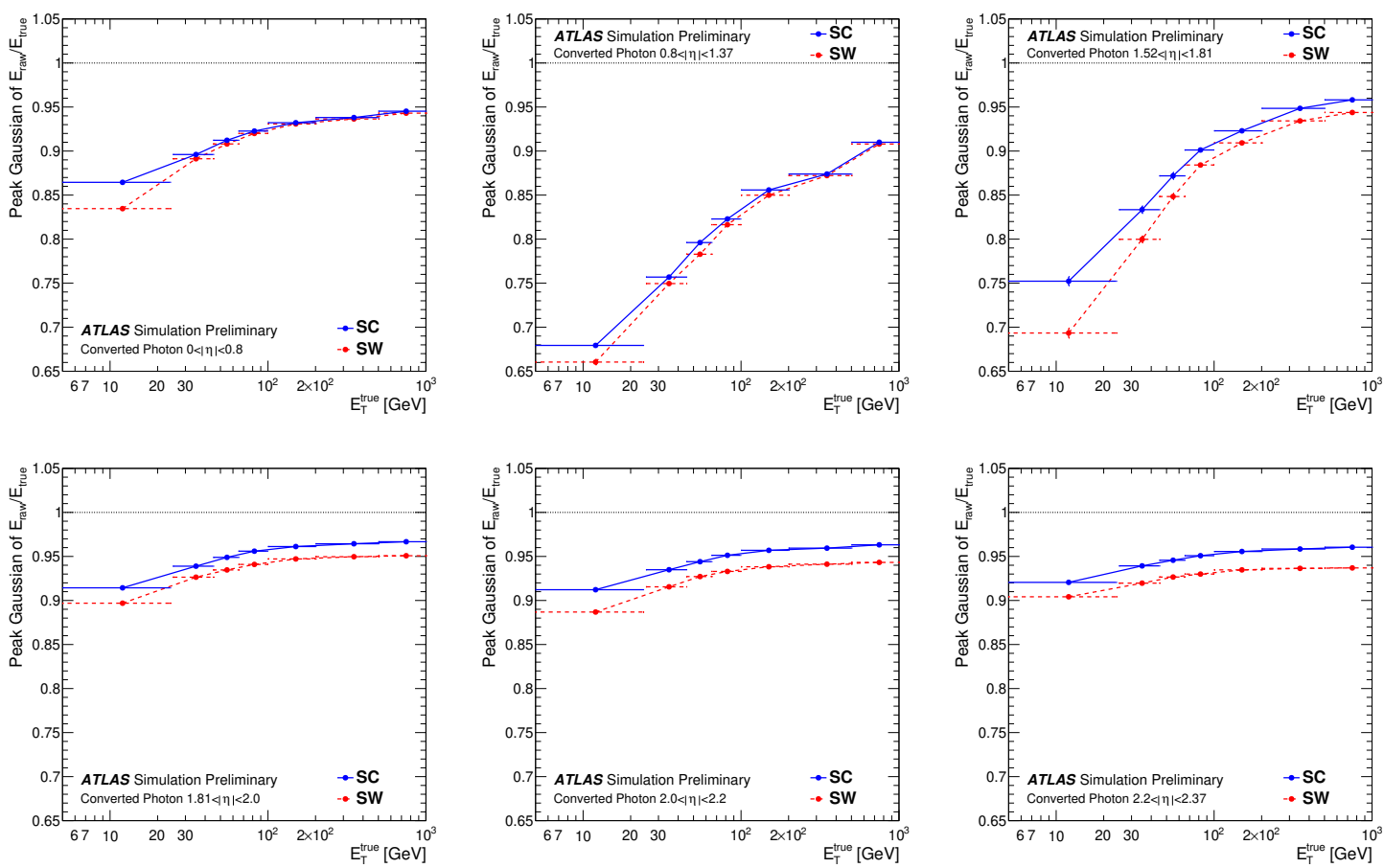

Figure 4.15: Comparison of uncalibrated Gaussian peak values for sliding window (red) and supercluster (blue) converted photons, using samples of single converted photon events. 
regions of $\left|\eta_{\text {true }}\right|$ and $E_{\mathrm{T}}^{\text {true }}$, while Figure 4.17 shows the fitted Gaussian peak for the same regions. The reconstructed unconverted photons in these distributions are required to be true primary photons, and pass loose photon quality requirements [81].

Unconverted photons are not subject to energy loss from bremsstrahlung radiation, and are uncharged particles, hence they do not leave tracks in the Inner Detector. So, unlike electrons and converted photons, it is not expected that the use of superclustering will consistently improve the resolution of unconverted photons. As shown in Figures 4.16 and 4.17, for barrel photons, there is little to no change in resolution observed between unconverted photons reconstructed using sliding window and supercluster approaches, although supercluster performance can degrade at very high $E_{\mathrm{T}}$. In some $|\eta|$ bins, supercluster unconverted photons are found to have slightly worse performance, although the difference in resolution is typically $\leq 5 \%$.

\subsection{MVA calibration}

For both electrons and photons, multivariate analysis (MVA) techniques are used to derive an energy calibration which corrects the uncalibrated energies of electron and photon candidates, implemented using the Toolkit for Multivariate Data Analysis (TMVA) [82]. As described in Refs. [73] and [83], the calibration is derived by training a multivariate regression to optimize the electron and photon energy response, while minimizing the root mean square resolution. The output of the regression is a correction factor which transforms the uncalibrated input energy $E_{\text {raw }}$ to a calibrated energy $E_{\text {cal }}$, such that $E_{\text {cal }} \sim E_{\text {true }}$. The MVA training is performed separately for electrons, converted photons, and unconverted photons. For each type of particle, simulated single particle samples are produced, with the particles passing through a 

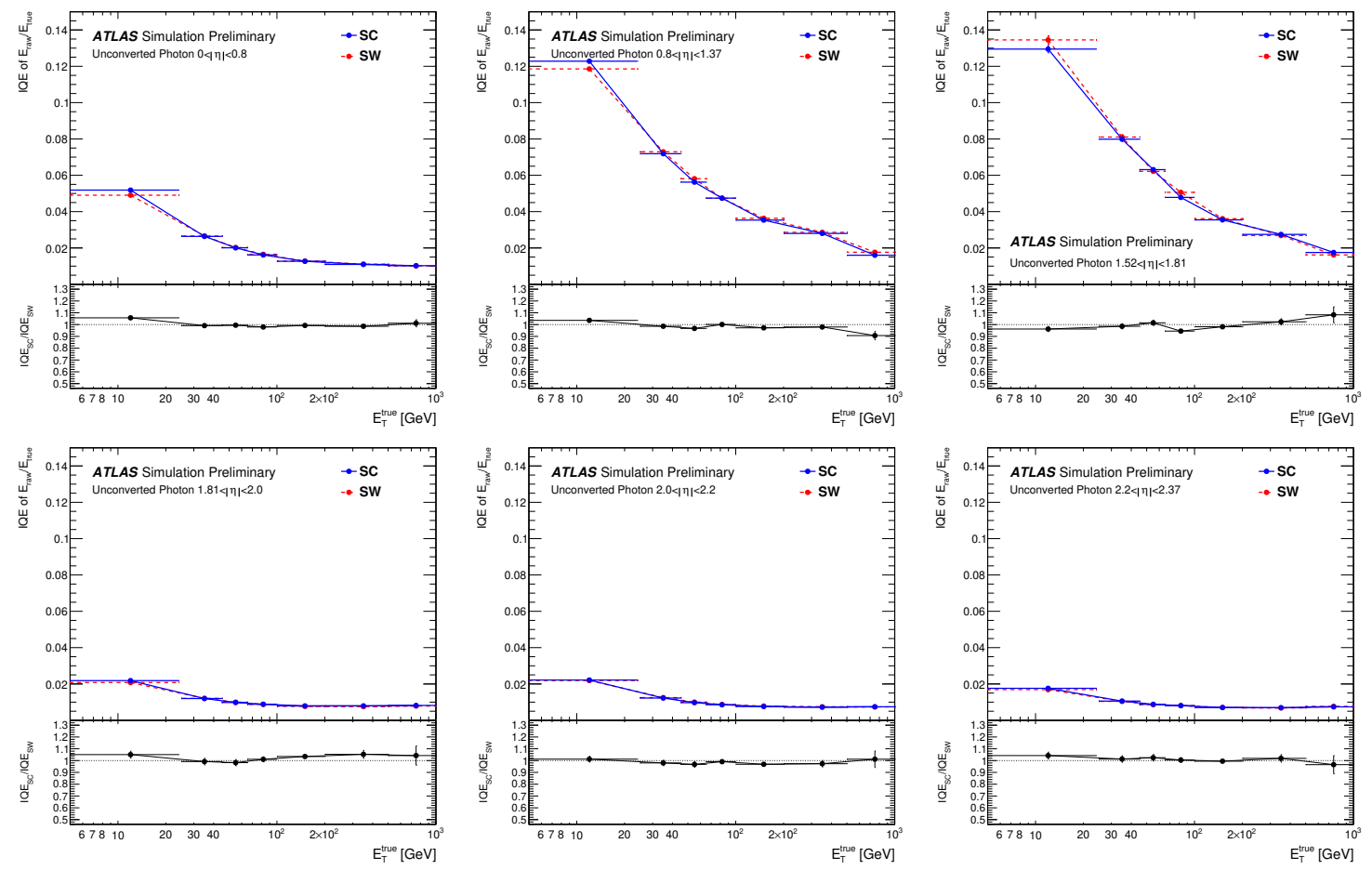

Figure 4.16: Comparison of uncalibrated IQE values between sliding window (red) and supercluster (blue) unconverted photons, using samples of single unconverted photon events.
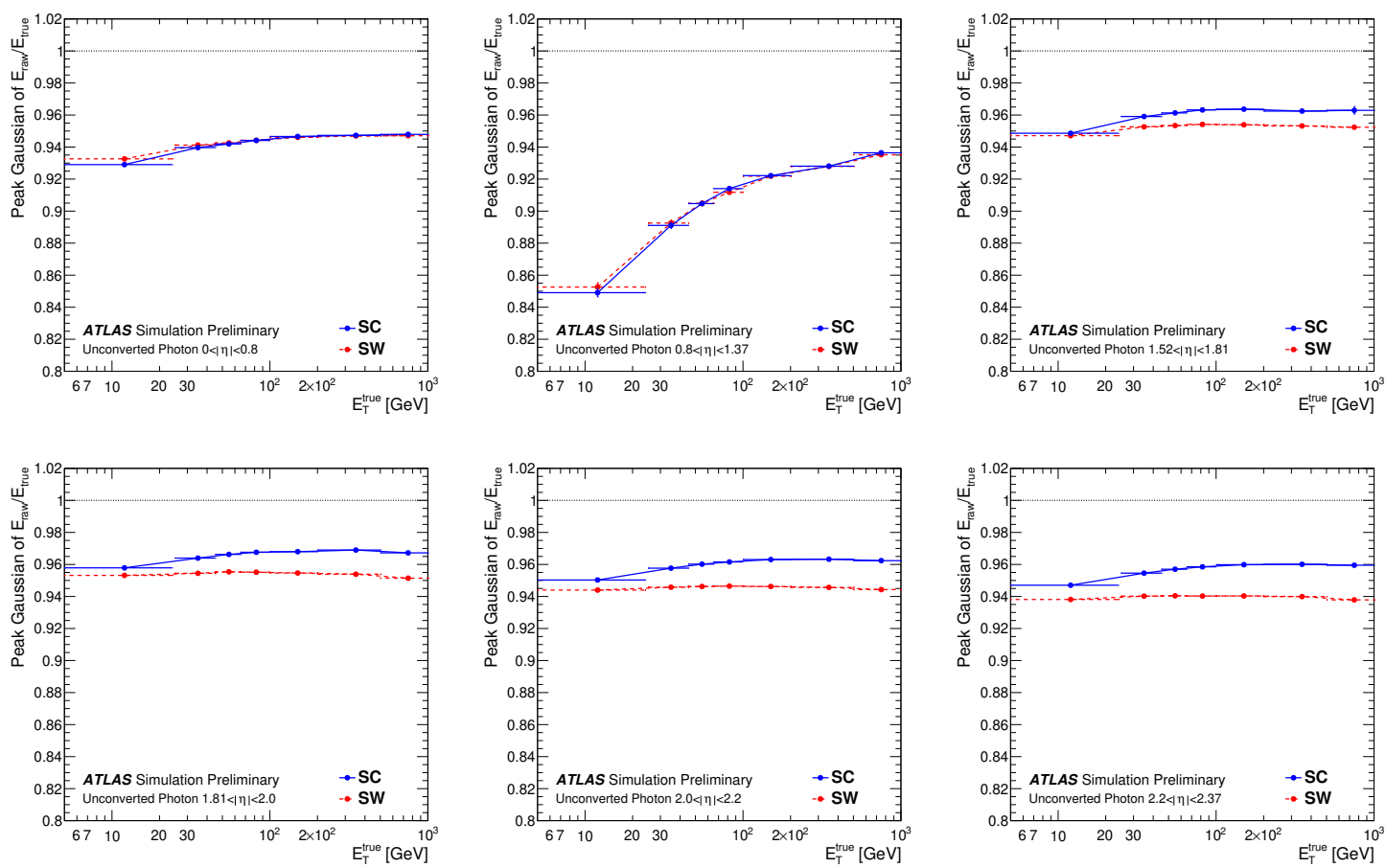

Figure 4.17: Comparison of uncalibrated Gaussian peak values for sliding window (red) and supercluster (blue) unconverted photons, using samples of single unconverted photon events. 
full ATLAS detector simulation, although no simulated pile-up noise is added. To derive the calibration, the samples are split into two roughly evenly-sized sub-samples, with one sub-sample used exclusively for training the regression, and the other used exclusively for testing. This splitting is done to avoid any bias in evaluating the calibrated energy response distributions, since one will "know" a priori the exact correction factors for the events composing the training sample.

The training variables used in the MVA calibration for electrons and photons are: the uncalibrated energy, $E_{\text {raw }}$; the ratio of the presampler energy to the calorimeter energy, or $f_{0}=E_{0} / E_{\text {raw }}$; the ratio of the energies measured in the first two layers of the calorimeter, $E_{\mathrm{L} 1} / E_{\mathrm{L} 2}$; the cluster barycentre pseudorapidity in the ATLAS coordinate system, $\eta_{\text {clus }}$; the cell index, or the distance of the cluster from $\eta=0$ in the calorimeter frame, in terms of the number of cells in the $\eta$-direction; and cluster barycentre variables related to $\eta$ and $\phi$ within the calorimeter frame. For the latter two variables, $\eta_{\text {Calo }}$ is given as the $\eta$ position with respect to the cell center in $\eta$, while $\phi_{\text {Calo }}$ is the $\phi$ position with respect to the absorber axis.

An important distinction regarding the final three variables is the choice of reference frame. In particular, the ATLAS coordinate system has its origin at the nominal interaction point, so with respect to this point, the calorimeter is displaced by a few $\mathrm{mm}$. Conversely, all calorimeter cells are in their nominal position in the calorimeter frame. Accordingly, the barycentre position will be slightly shifted depending on whether it is represented in the ATLAS or calorimeter frames [83].

Photons are considered converted if they are matched to a conversion vertex with radius $R_{\text {conv }}$ smaller than $800 \mathrm{~mm}$. For these converted photons, $R_{\text {conv }}$ is used as an additional input to the training only if the vectorial sum of the conversion track trans- 
verse momenta is above $3 \mathrm{GeV}$. For converted photons built using tracks containing at least one hit in either the Pixel or SCT detectors, additional quantities are used as training variables: $E_{\mathrm{T}}^{\text {raw }} / p_{\mathrm{T}}^{\text {conv }}$, where $p_{\mathrm{T}}^{\text {conv }}$ is the vectorial sum of the conversion track transverse momenta; and the fraction of the conversion transverse momentum carried by the highest- $p_{\mathrm{T}}$ track, $p_{\mathrm{T}}^{\max } / p_{\mathrm{T}}^{\text {conv }}$.

The shapes of the variables common to the electron and photon trainings are shown in Figure 4.18 for the new and previous $e / \gamma$ reconstruction algorithms. Good agreement is observed between the two algorithms for each training variable, thus validating their continued usage in the MVA calibration. Distributions of variables used only for the calibration of converted photon clusters are shown in Figure 4.19, further demonstrating good agreement between the two algorithms.

\subsection{Calibrated supercluster performance}

The following sections detail the energy response of electrons and photons after calibration is applied, as performed using samples of single particles reconstructed both via sliding window and supercluster-based approaches. Analogous to the uncalibrated results, the metric of comparison between the previous and new reconstruction algorithms is the ratio of the calibrated cluster energy to the true particle energy, $E_{\text {cal }} / E_{\text {true }}$. In general, the MVA calibration is performed using training and testing samples which contain no simulated pile-up, so the following results are presented without simulated pile-up. It is worth noting, as well, that the peaks of the energy response distributions $E_{\text {cal }} / E_{\text {true }}$ in each $E_{\mathrm{T}}$ and $|\eta|$ bin show no deviations from the expected $E_{\text {cal }} / E_{\text {true }}=1$ above $\sim 0.5 \%$. 

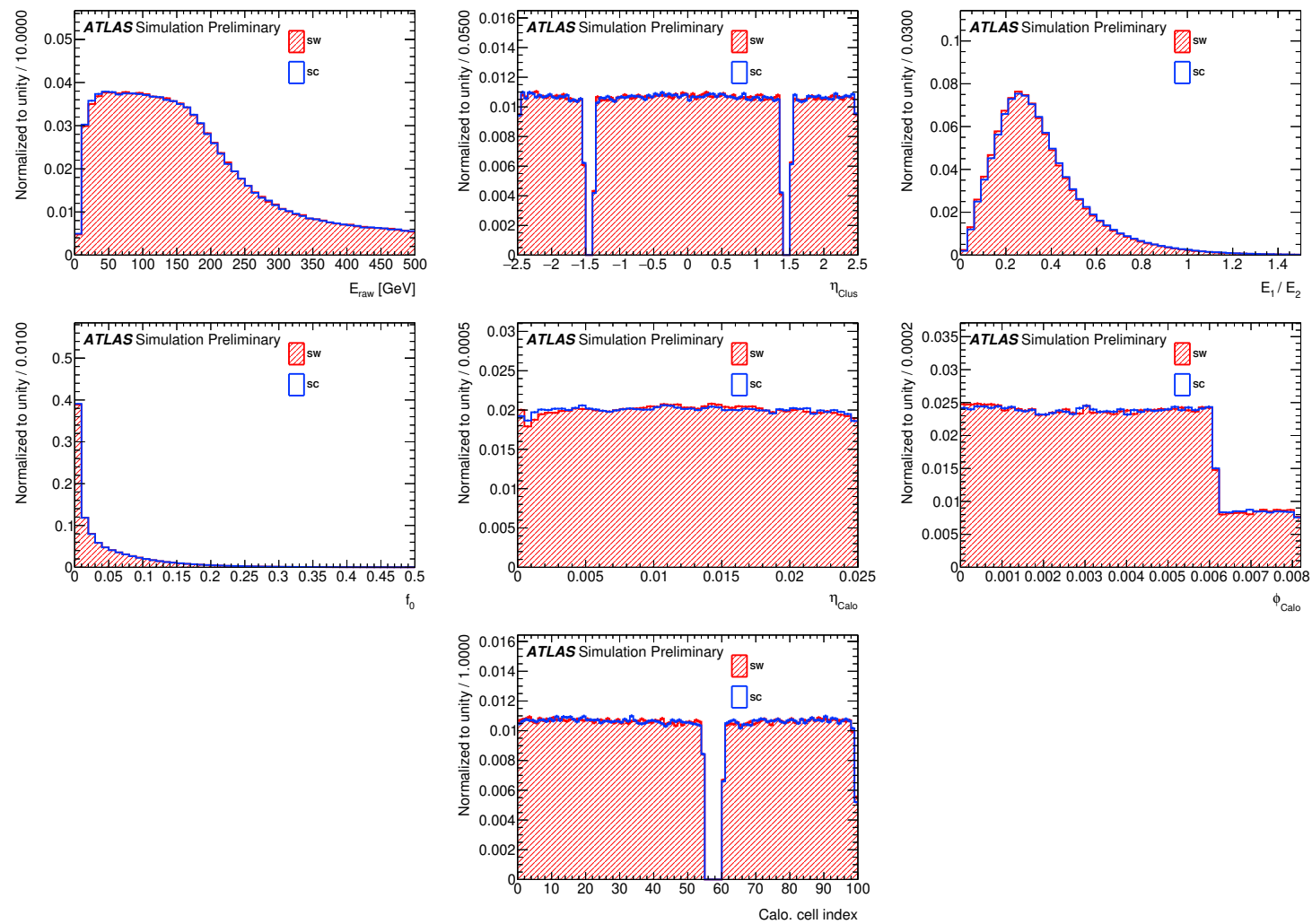

Figure 4.18: Distributions of cluster-based MVA calibration training variables for sliding window and supercluster-based electrons and photons.
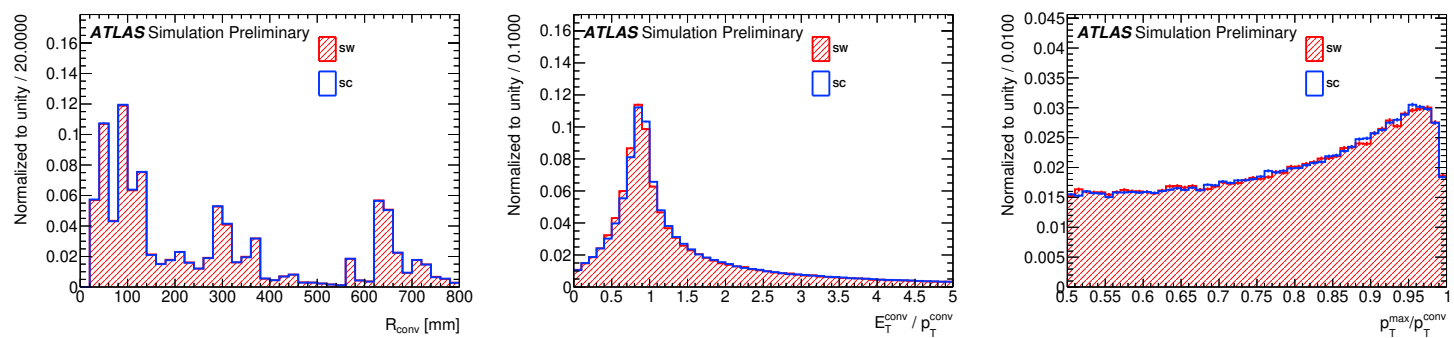

Figure 4.19: Distributions of track-based MVA calibration training variables for sliding window and supercluster-based converted photons using the new reconstruction software. 


\subsubsection{Electrons and converted photons}

Comparisons of the calibrated energy response of simulated single electrons built using sliding window and supercluster-based approaches are given in Figure 4.20, comparing the IQE of the two methods in different regions of $\left|\eta_{\text {true }}\right|$ and $E_{\mathrm{T}}^{\text {true }}$. The reconstructed electrons in these distributions are required to be true primary electrons, and pass loose electron quality requirements. After calibration, the supercluster algorithm retains a good improvement in resolution compared to the previous method. In particular, improvements of $20-30 \%$ in resolution are found in some bins in the end-cap region of the detector, as well as in the central region for low $E_{\mathrm{T}}$ electrons.

Comparisons of the calibrated response of converted photons built using sliding window and supercluster-based approaches are given in Figure 4.21. The reconstructed converted photons in these distributions are required to be true primary photons, and pass loose photon quality requirements. Good improvements in the resolution can also be seen for converted photons, with the improvement in some bins being over $30 \%$ relative to the previous algorithm.

\subsubsection{Unconverted photons}

Comparisons of the calibrated response of unconverted photons built using sliding window and supercluster-based approaches are given in Figure 4.22. In particular, Figure 4.22 shows a comparison of the IQE of the two methods in different regions of $\left|\eta_{\text {true }}\right|$ and $E_{\mathrm{T}}^{\text {true }}$. The reconstructed unconverted photons in these distributions are required to be true primary photons, and pass loose photon quality requirements. As with the uncalibrated energy response, the overall change in performance is small, due to the generally narrower shower width expected of unconverted photons, and lack of 

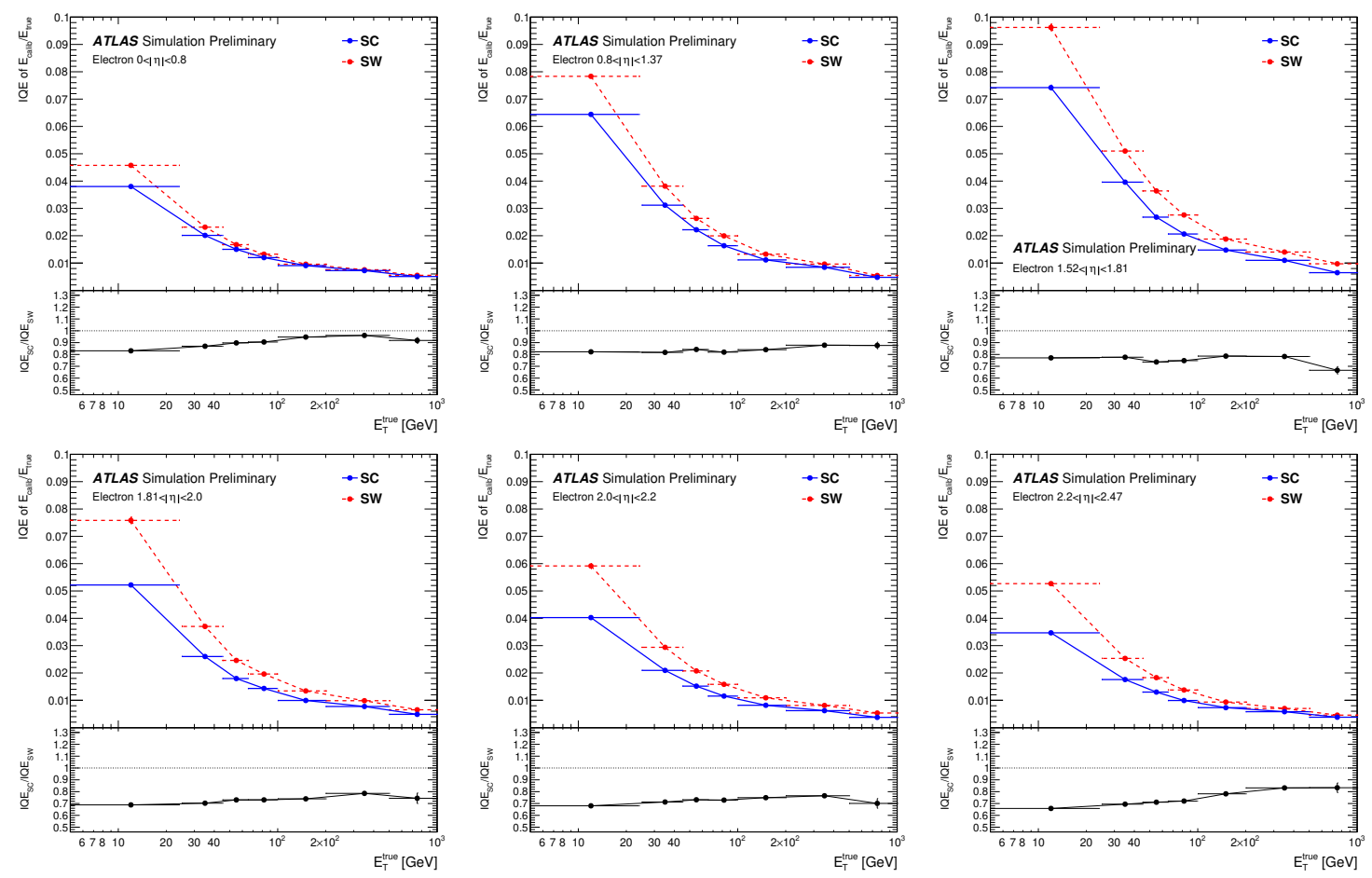

Figure 4.20: Comparison of calibrated IQE values between sliding window (red) and supercluster (blue) electrons, using samples of single electron events.
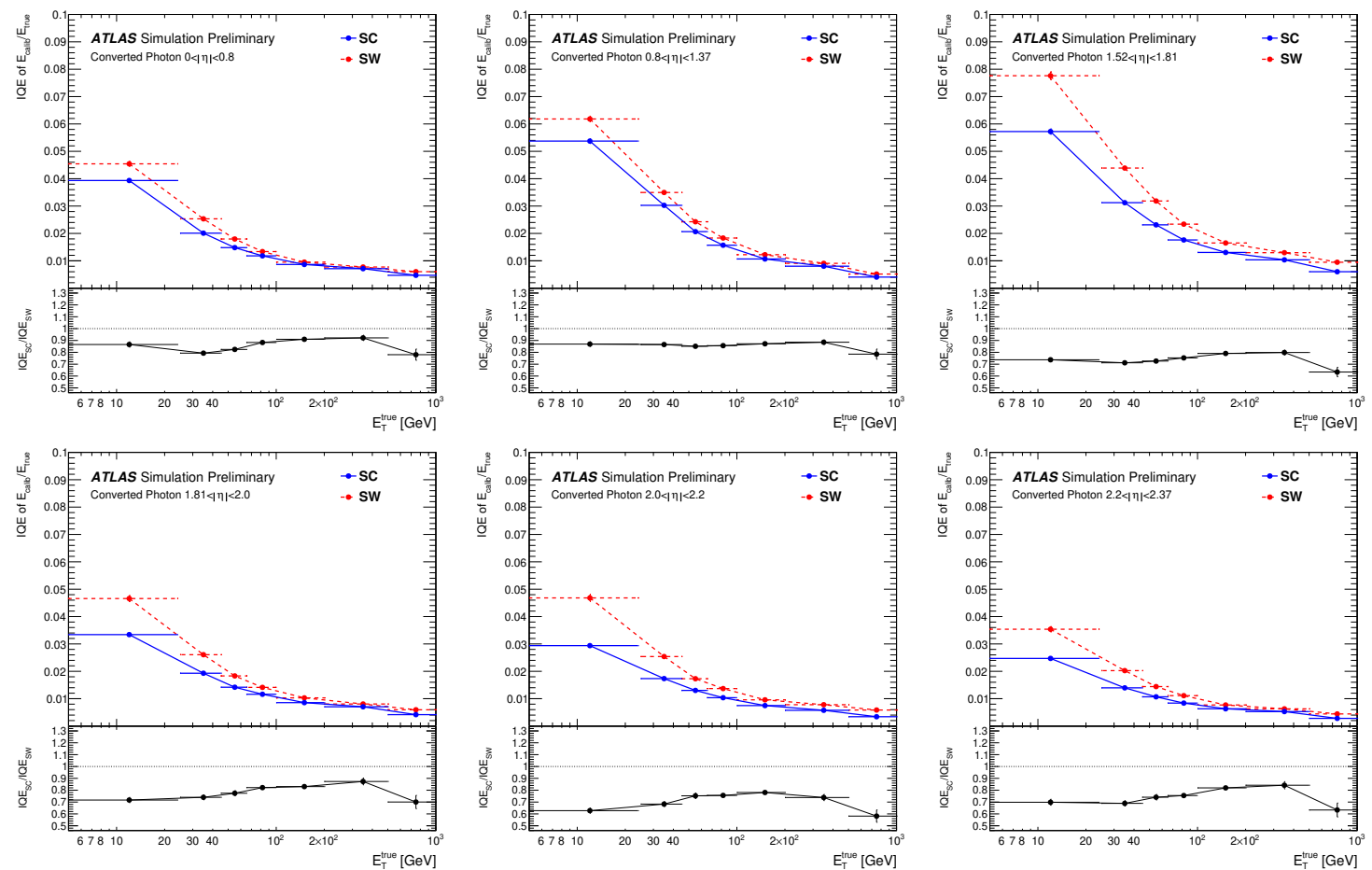

Figure 4.21: Comparison of calibrated IQE values between sliding window (red) and supercluster (blue) converted photons, using samples of single converted photon events. 

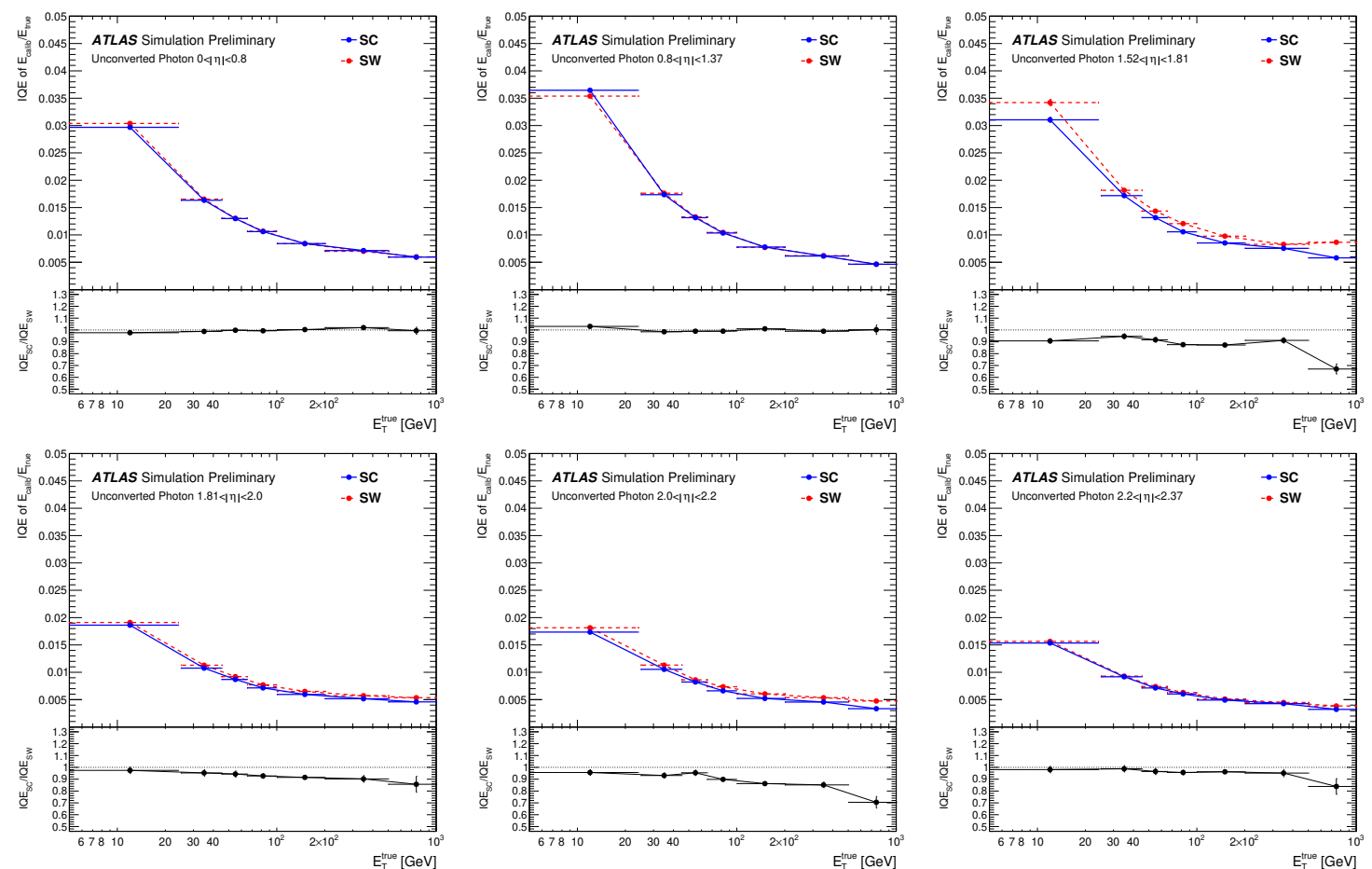

Figure 4.22: Comparison of calibrated IQE values between sliding window (red) and supercluster (blue) unconverted photons, using samples of single unconverted photon events.

bremsstrahlung for unconverted photons. However, some improvement in calibrated resolution is observed for high $E_{\mathrm{T}}$ bins in the end-cap region.

\subsection{Supercluster performance with simulated pile- up interactions}

An important consideration when using a topo-cluster based reconstruction is the sensitivity of the energy response to the presence of additional clusters from pileup interactions. The noise thresholds for topo-cluster reconstruction must be set to a level which is appropriate for the expected luminosity and detector conditions, 
meaning that the thresholds tend to be set based on empirical studies. If the noise thresholds are set too low, electron or photon clusters from HS interactions may accumulate cells from unrelated clusters which originate in pile-up interactions, leading to an overestimate of the cluster energy, as well as a degraded energy resolution. Spurious electrons or photons may also be reconstructed using clusters that originate purely from pile-up noise. Conversely, if the thresholds are set too high, then the accumulation of cluster cells will be limited, which can lead to an underestimation of cluster energies, or worse, a reduced efficiency for low energy particles.

To study the effects of pile-up on the electron and photon energy response, samples of simulated single particles were generated using "nominal" topo-cluster noise thresholds tuned for $\langle\mu\rangle \sim 40$. Events were generated under different luminosity conditions, with each event having a possible average number of interactions per bunch crossing in the range $0<\langle\mu\rangle<60$. The simulated electrons and photons were further required to pass loose quality requirements, and have $E_{\mathrm{T}}>7 \mathrm{GeV}$. Calibrated energy response distributions were then produced for the barrel and end-cap detector regions in slices of $\langle\mu\rangle$ demarcated by $[0,15,30,45,60]$, with the resolution computed based on the energy response distribution in each $\langle\mu\rangle$ slice.

Figure 4.23 shows the energy resolution in slices of $\langle\mu\rangle$ for calibrated electron clusters in different truth $|\eta|$ and $E_{\mathrm{T}}$ regions. Likewise, Figures 4.24 and 4.25 show the same distributions for calibrated converted and unconverted photon clusters, respectively. The position of the Gaussian fit in each case remains constant around $E / E_{\text {true }} \sim 1$ with successively higher $\langle\mu\rangle$, so these distributions are not shown. It is evident that with increasing $\langle\mu\rangle$, the performance of both clustering algorithms degrades due to cluster cells containing more noise on average, in addition to the 
true shower energy. Therefore, the increased noise levels are the dominant reason for performance degradation. However, an important conclusion that can be drawn from Figures 4.23 and 4.24 is that for electron and converted photon superclusters, the improvements in energy resolution are largely maintained at higher levels of $\langle\mu\rangle$.

A secondary effect is visible for superclusters built when $\langle\mu\rangle \sim 45$ or so, in that the relative improvements in resolution become smaller with respect to the sliding window method, particularly in the end-cap region and high $E_{\mathrm{T}}$, where tracking performance is more limited. Such a result is not entirely unexpected; because the topo-cluster noise thresholds are tuned to cover mainly $\langle\mu\rangle \leq 40$, the increased noise levels are compounded by the gradual acceptance of additional cells during topocluster reconstruction when $\langle\mu\rangle>40$. Unconverted photons, in particular, produce narrow showers which do not spread in the $\phi$-direction from material interactions, so allowing the cluster to grow in this manner tends to result in the acquisition of extra noise at higher pile-up, rather than more true shower energy. Since the noise thresholds for a given data taking period are adjusted according to the expected $\langle\mu\rangle$, a re-tuning of the thresholds for higher pile-up would likely reproduce the performance improvements of lower $\langle\mu\rangle$.

One future point of investigation is the use of pile-up sensitive variables in the MVA calibration training, which may provide a calibration that can adapt as necessary to higher luminosity conditions, thereby maintaining the improvement in calibrated energy resolution over a wide range of $\langle\mu\rangle$. A modification of the calibration methodology may be necessary for unconverted photons, which in general show more sensitivity to higher pile-up the calibrated energy response. It is also possible that tuning the supercluster formation for clusters with no associated tracks may allow for 
a reduced sensitivity to higher pile-up, since such particles are unambiguously defined as unconverted photons in the reconstruction algorithm.

\subsection{Supercluster performance in relevant physics topologies}

So far, the performance of the new reconstruction algorithm has been evaluated using single particles in isolation, and in the presence of simulated pile-up. However, the $e / \gamma$ reconstruction ultimately serves as input to several analyses which target precision measurements, rare searches, properties measurements (spin, $\mathcal{C P}$, couplings, etc.), as well as tests for new physics. Therefore, the new reconstruction algorithm was applied to simulations of several relevant physics topologies, including those used for precision physics analyses, as well as Higgs boson physics analyses. Comparisons were also made to simulated samples reconstructed using the previous iteration of the software, in order to gauge the improvement brought by the new reconstruction.

\subsection{1 $J / \psi \rightarrow e^{+} e^{-}$and $Z \rightarrow e^{+} e^{-}$}

The performance of the supercluster algorithm in $p p \rightarrow J / \psi \rightarrow e^{+} e^{-}$decays yields critical information on how the new reconstruction performs for low-mass resonances, as well as low $p_{\mathrm{T}}$ electrons in general. Simulated $J / \psi \rightarrow e^{+} e^{-}$events were produced using the PowHEg generator [84], with the UE and MPI components simulated using Pyтhia 8 [85]. The simulated data were reconstructed using the previous reconstruction software and MVA calibration based around sliding window electrons, as well as the latest reconstruction software and calibration using superclusters. Dielectron $J / \psi$ 

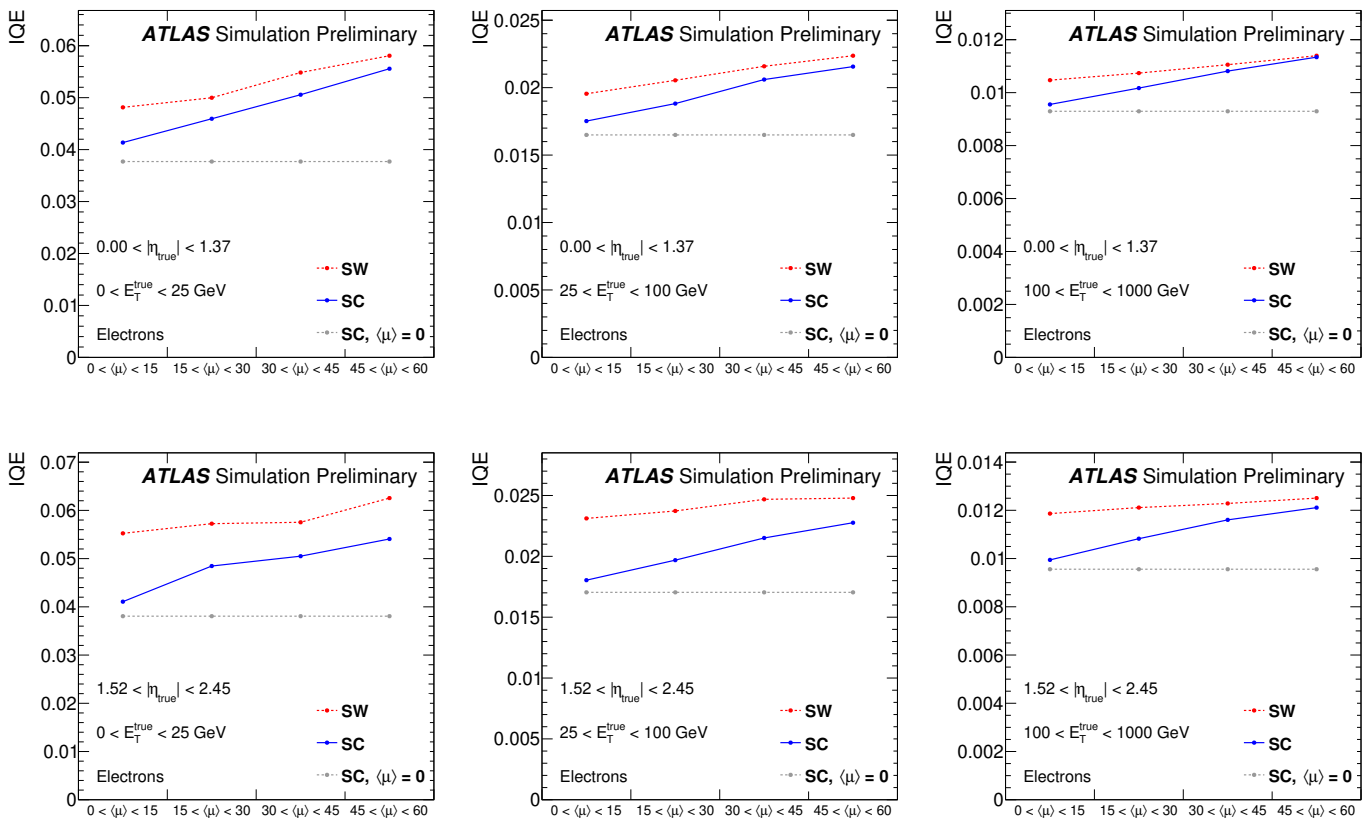

Figure 4.23: Calibrated energy response resolution, expressed in terms of IQE, for simulated single electrons at different $\langle\mu\rangle$ levels. Sliding window-based response is shown in red, while the supercluster-based response is shown in blue.
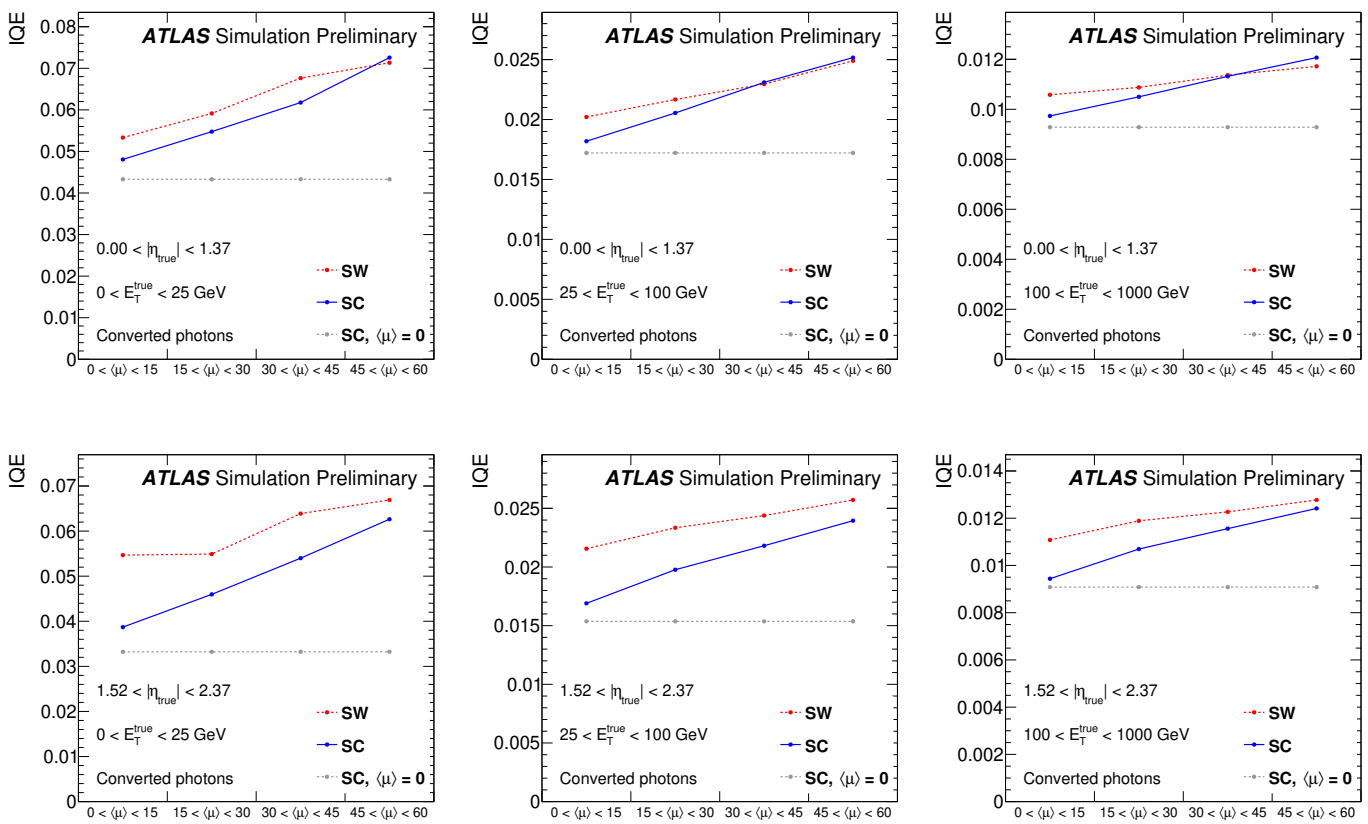

Figure 4.24: Calibrated energy response resolution, expressed in terms of IQE, for simulated single converted photons at different $\langle\mu\rangle$ levels. Sliding window-based response is shown in red, while the supercluster-based response is shown in blue. 

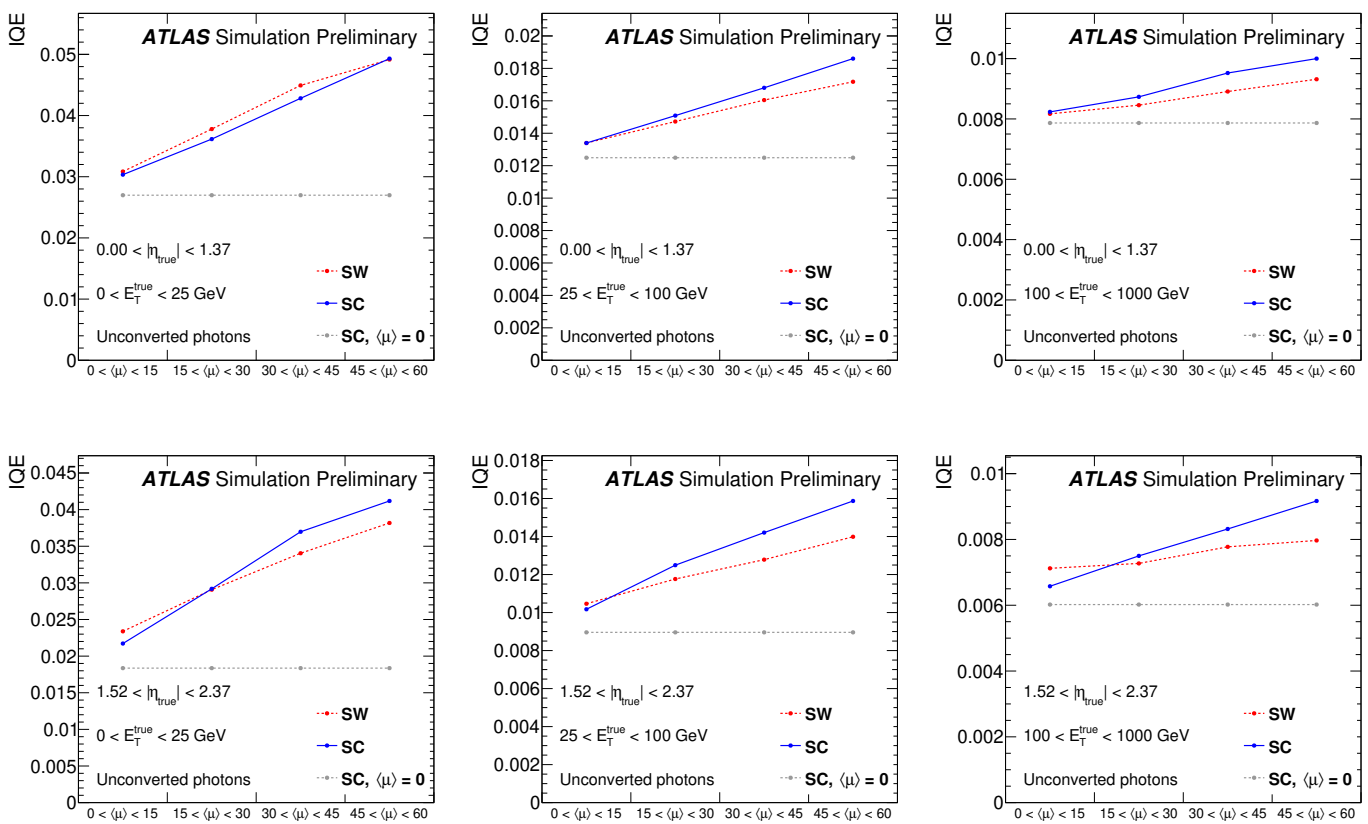

Figure 4.25: Calibrated energy response resolution, expressed in terms of IQE, for simulated single unconverted photons at different $\langle\mu\rangle$ levels. Sliding window-based response is shown in red, while the supercluster-based response is shown in blue. 


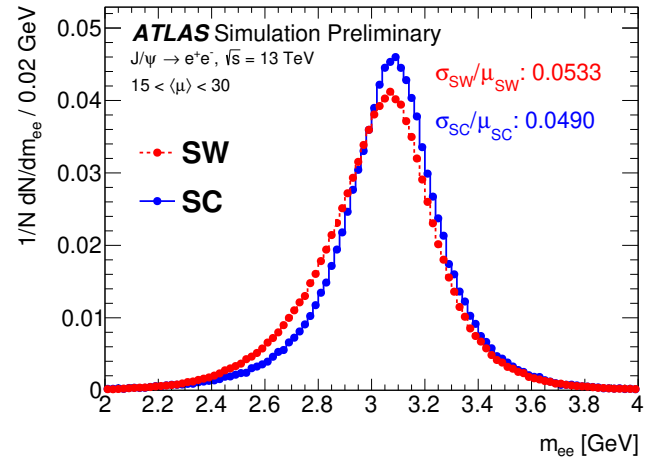

(a) $J / \psi \rightarrow e^{+} e^{-}$

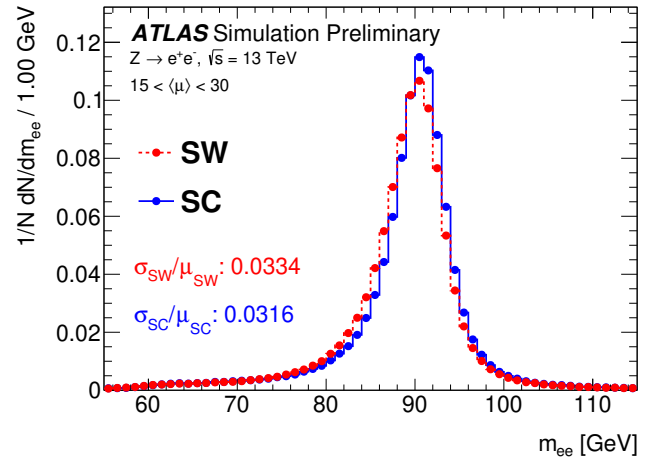

(b) $Z \rightarrow e^{+} e^{-}$

Figure 4.26: Invariant mass distribution of the dielectron system from simulations of reconstructed $J / \psi \rightarrow e^{+} e^{-}$decays (left) and $Z \rightarrow e^{+} e^{-}$decays (right).

pair candidates were formed using the two highest $p_{\mathrm{T}}$ electrons that pass loose quality requirements. Furthermore, the $J / \psi$ boson candidate events were required to have $15<\langle\mu\rangle<30$, as the sliding window samples used were produced for lower luminosity conditions, such that $\langle\mu\rangle<30$. Accordingly, studying the region $15<\langle\mu\rangle<30$ provided a roughly equivalent distribution of $\langle\mu\rangle$ in each scenario.

Distributions of the reconstructed dielectron invariant mass $m_{e^{+} e^{-}}$are shown for supercluster and sliding window-based reconstruction algorithms in Figure 4.26. It is evident from the distribution of $m_{e^{+} e^{-}}$that the supercluster algorithm improves the mass response of the $J / \psi$ peak, providing a narrower resonance with a smaller radiative tail, indicating a compensation for bremsstrahlung-related energy losses. The change in resolution $(\sigma)$ and mean $(\mu)$ are quantified using $\sigma / \mu$, as determined using a Gaussian fit to the core of the $m_{e^{+} e^{-}}$distribution. Using this approach, an improvement of $9.2 \%$ is observed in $\sigma / \mu$ through the use of superclusters. A small shift towards the best-known $J / \psi$ mass is also observed for the supercluster $m_{e^{+} e^{-}}$distribution, with the peak of the fitted Gaussian found at $3.08 \mathrm{GeV}$ for the 
supercluster distribution, and $3.05 \mathrm{GeV}$ for the sliding window distribution.

Another important performance consideration is how the reconstruction algorithm responds for $p p \rightarrow Z \rightarrow e^{+} e^{-}$decays, which has direct implications for precision Standard Model physics measurements in ATLAS. To this end, simulated $Z \rightarrow e^{+} e^{-}$ decays were generated similarly using Powheg interfaced to PYThiA 8, and reconstructed using the previous reconstruction software and MVA calibration based around sliding window electrons, as well as the latest reconstruction software and calibration using superclusters. Dielectron $Z$ pair candidates were formed using the two highest $p_{\mathrm{T}}$ electrons that pass loose quality requirements. Furthermore, in order to make a fair comparison of luminosity conditions between the two samples, the $Z$ boson candidate events were also required to have $15<\langle\mu\rangle<30$.

The reconstructed dielectron invariant mass spectra are shown for supercluster and sliding window-based reconstruction algorithms in Figure 4.26. It is evident from the distribution of $m_{e^{+} e^{-}}$that the supercluster algorithm improves the mass response of the $Z$ boson peak, providing a narrower resonance with a smaller radiative tail, indicating a compensation for bremsstrahlung-related energy losses. The improvement in resolution and mean, quantified using $\sigma / \mu$ from a Gaussian fit about the peak of the $m_{e^{+} e^{-}}$distribution, is $\sim 5 \%$ when using supercluster electrons. The peak of the supercluster mass distribution is also found at $90.6 \mathrm{GeV}$, which is $500 \mathrm{MeV}$ closer to the true $Z$ boson mass at $91.1876 \mathrm{GeV}$ than the sliding window equivalent.

\subsection{2 $H \rightarrow Z Z^{*} \rightarrow 4 \ell$}

A particularly important consideration in the move to the new electron reconstruction algorithm is the effect on the $H \rightarrow Z Z^{*} \rightarrow \ell^{+} \ell^{-} \ell^{+} \ell^{-}$channel, which is considered 
the "golden" Higgs boson decay channel due to its high signal-to-background ratio, the resolution of its final state particles (muons, electrons), and the ability to fully reconstruct the $4 \ell$ system. The $4 \ell$ candidate selection in the $H \rightarrow Z Z^{*} \rightarrow 4 \ell$ analysis tends to feature a rather inclusive selection of electrons, with loose electrons above $p_{\mathrm{T}}>7 \mathrm{GeV}$ allowed to be included in Higgs boson candidates. Accordingly, the resolution improvements brought for low $p_{\mathrm{T}}$ and end-cap electrons should lead to an improvement in the mass resolution of reconstructed $4 \ell$ candidates.

To examine the effects of the new reconstruction algorithm on the invariant mass resolution of $4 \ell$ candidates, samples of simulated VBF-mediated Higgs boson signal were produced using Powheg and Pythia8 with a Higgs boson mass of $m_{H}=$ $125 \mathrm{GeV}$, with PythiA also simulating the $H \rightarrow Z Z^{*} \rightarrow 4 \ell$ decays. The events were passed through a full detector simulation in GEANT4, along with simulated pileup interactions which emulated the luminosity conditions of the 2016 data taking period. The simulated events were then reconstructed with the ATLAS software, using both sliding window and supercluster-based algorithms.

The $4 \ell$ candidate reconstruction and selection generally proceeded identically to previous analyses [86]. The resultant distributions of $m_{4 \ell}$, as reconstructed using sliding window and supercluster-based approaches, are given in Figure 4.27 for each electron-related decay channel - namely, $2 \mu 2 e, 2 e 2 \mu$, and $4 e$, where the first $2 \ell$ in the channel name denotes the higher $p_{\mathrm{T}}$ lepton pair. Each channel shows a visible improvement in both the resolution, as well as the position of the peak, which is directly attributable to the changes in the electron reconstruction. In particular, the supercluster-based $m_{4 \ell}$ distributions are found to be narrower than the sliding window equivalent, and peak closer to the true Higgs boson mass of $m_{H}=125 \mathrm{GeV}$. 
To quantify the change in peak position and resolution, the invariant mass distributions for each channel were fit with two Crystal Ball functions with the same mean and width, which allows the fit to capture both the peak and the tails of the distributions, with the fitted resolution given in each plot in Figure 4.27. From these fits, the improvement in $\sigma / \mu$ for each channel is found to be $3-5 \%$, with the shift towards the true Higgs boson mass being $0.5-1 \mathrm{GeV}$ for each case. The pattern of improvement in the $4 \ell$ mass resolution mirrors the previous studies done using single particles; when the electron pair has the highest momentum, the improvement in resolution is a few percent, as the supercluster and sliding window algorithms tend to converge to the same energy resolution at high $E_{\mathrm{T}}$ (or, in practice, $p_{\mathrm{T}}$ ). Conversely, the channels featuring lower $p_{\mathrm{T}}$ electrons demonstrate larger improvements of $\mathcal{O}(5 \%)$ in mass resolution, owing to the fact that the supercluster algorithm shows the most improvement for low $E_{\mathrm{T}}$ electrons. 

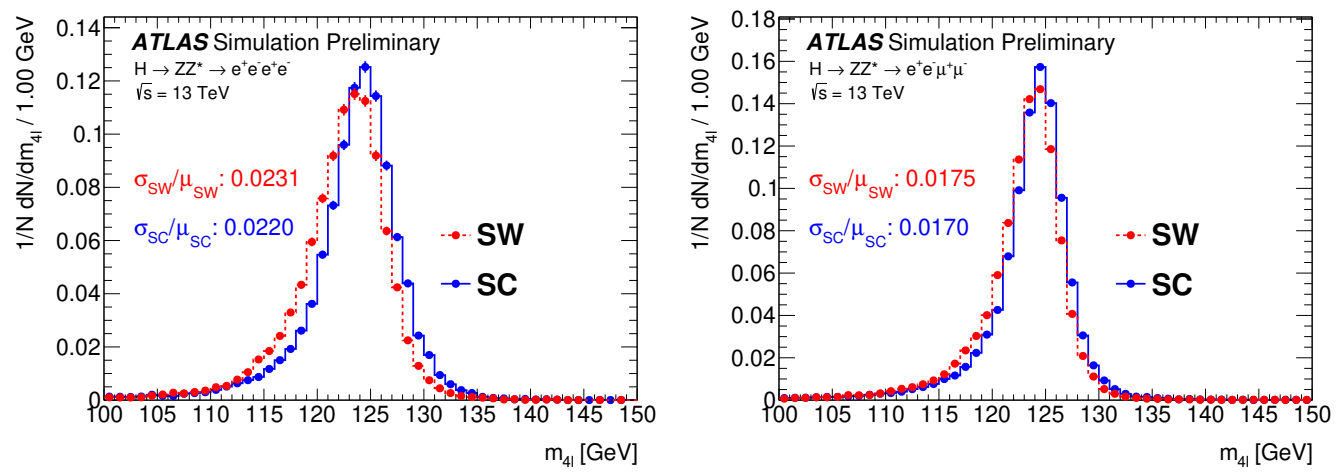

(a) $4 e$

(b) $2 e 2 \mu$

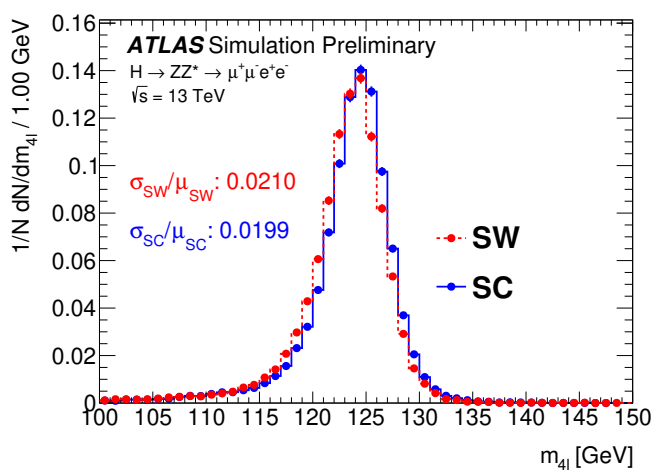

(c) $2 \mu 2 e$

Figure 4.27: Superimposed distributions of the invariant mass of simulated reconstructed four lepton candidates using $H \rightarrow Z Z^{*} \rightarrow 4 \ell$ analysis selection. The resolution values are derived from the width of a fitted Double Crystal Ball function. 


\section{Chapter 5}

\section{Modelling Higgs boson production}

\section{and decays}

The generation of theoretically accurate predictions for a signal process is a critical component of any analysis focused on identifying rare (or new) physics processes. Much attention is paid to properly modelling Higgs boson production and decay, due to the fact that the experimentally cleanest processes tend to be quite rare, while the more abundant processes are overwhelmed by QCD or electroweak backgrounds. A particular focus must be paid to signal processes which overlap in some kinematic regime, as one process can in principle serve as a "background" or "contaminant" of another. Accurate modelling of Higgs signal is particularly important for studies in the $H \rightarrow Z Z^{*} \rightarrow 4 \ell$ channel, where the signal-to-background ratio can be as high as $S / B \sim 7$ when two or more final state jets are present.

Due to the heavy reliance on simulations to understand the processes of interest in collider physics experiments, it is worth introducing here some important simulationrelated terminology used in later sections. Most expected results in ATLAS are 
derived from simulations based on the Monte Carlo (MC) method [87], with software that produces such predictions termed a Monte Carlo generator. An important consideration is how the detector will respond to physical interactions mediated by $p p$ collisions. To understand the behaviour of such processes, generators are used which simulate the HS interaction between two protons, such as Powheg [84]. These generators produce what may be termed parton-level samples, containing stable leptons, gauge bosons, quarks, and gluons.

At this point, one must consider that stable weak bosons, quarks, and gluons are never observed in nature. So, in addition to the HS interaction, one must also employ a generator which simulates the UE and MPI components of a $p p$ collision, for which Shower Monte Carlo (SMC) programs such as PyThia [85] are used. The HS and SMC generators interface through standardized records of particle energies, positions, and momenta before and after the $p p$ collision. Through the use of an SMC generator, physical phenomena can be introduced which are otherwise practically impossible to model at parton-level, such as initial/final state radiation (ISR/FSR), and quark or gluon hadronization, producing truth-level samples. The SMC generators also tend to simulate decays of weak bosons and the Higgs boson, with some notable exceptions (see Section 5.1.2 for more details).

With these aspects of the simulation in mind, this chapter presents an overview of the different methods that are used to model the production and decay of Higgs bosons in ATLAS, with a particular emphasis on the $H \rightarrow Z Z^{*} \rightarrow 4 \ell$ analysis. Furthermore, effective field theories are introduced as a means to probe for the presence of anomalous Higgs boson couplings, emphasizing the effects of different BSM operators on Higgs signal predictions, and how a potentially infinite coupling parameter 


\begin{tabular}{ll}
\hline \hline Order QCD & $\sigma_{\mathrm{ggF}}[\mathrm{pb}]$ \\
\hline $\mathrm{LO}$ & $16.56_{-17.5 \%}^{+21.5 \%}$ \\
$\mathrm{NLO}$ & $32.22_{-17.9 \%}^{+18.4 \%}$ \\
$\mathrm{NNLO}$ & $44.14_{-8.1 \%}^{+7.6 \%}$ \\
$\mathrm{~N}{ }^{3} \mathrm{LO}$ & $48.58_{-6.7 \%}^{+4.6 \%}$ \\
\hline \hline
\end{tabular}

Table 5.1: Partonic cross sections computed at different QCD orders for inclusive, gluon fusionmediated Higgs production with $m_{H}=125 \mathrm{GeV}$ and $\sqrt{s}=13 \mathrm{TeV}$. The LO and NLO cross section estimates were computed using MADGRAPH5_aMC@NLO.

space may be efficiently probed with a finite number of samples.

\subsection{Gluon fusion Higgs production}

The ggF Higgs boson production process is one of the more complex interactions to model, and as such, has received significant attention from the community of theoretical physicists focused on Higgs boson-related MC methods. The reason for this complexity is that ggF proceeds not through a direct coupling of gluons to the Higgs boson at tree-level, but rather through loops of heavy quarks. Accordingly, higher-order QCD corrections to this process occur in the $g q \bar{q}$ vertices, internally within the lines of the $q \bar{q}$ loop, and by self-interactions of gluons. The effects of these higher-order corrections are quite remarkable; as shown in Table 5.1, the state-of-theart $\mathrm{N}^{3} \mathrm{LO}$ QCD cross section for $\mathrm{ggF}$ at $\sqrt{s}=13 \mathrm{TeV}$ [31] is roughly $300 \%$ larger than the LO prediction for a Higgs boson with mass $m_{H}=125 \mathrm{GeV}$.

As the strengths of Higgs boson couplings are proportional to particle mass, the ggF interaction is primarily mediated by top-anti-top quark loops. In general, the evaluation of the matrix element for such a process can be computationally difficult 
beyond LO in QCD, so a simplified approach is often taken which frames the gluon fusion process as an effective Higgs-gluon contact interaction, otherwise known as the "heavy top" limit [88]. Here, the top quark is assumed to have infinite mass, and the approximation is generally valid for a region $m_{H}<2 m_{t}$. However, above this region in $m_{H}$, or for a high $p_{\mathrm{T}}$ Higgs boson, non-trivial differences in the kinematic spectra can emerge, as the Higgs boson can become much more energetic than the threshold set by the top mass. In this work, several MC generators are used to model ggF Higgs production for specific purposes, some of which include quark mass effects, with others employing the heavy top approximation. Where appropriate, theoretical uncertainties are derived to account for the effects of a finite top quark mass on the Higgs $p_{\mathrm{T}}$ spectrum, as described further in Section 8.2.1. The following sections present an overview of the methods used in this work to simulate ggF Higgs production, particularly as they relate to the $H \rightarrow Z Z^{*} \rightarrow 4 \ell$ decay channel.

\subsubsection{NNLO production via the POWHEG NNLOPS process}

Currently, it is possible to generate samples of ggF events at NNLO QCD, including quark mass effects, using the Powheg NnLops method [89]. The NnLOPS approach starts with a sample of $H+1 j$ events generated at NLO QCD using the Powheg HJ generator, which implements the Multi-Scale Improved NLO (MiNLO) prescription [90]. For each event in the $H+1 j$ sample, a correction factor is applied according to the Higgs boson rapidity $y_{H}$. These correction factors are derived from the HNNLO [91] generator, which models inclusive ggF-mediated Higgs boson production at NNLO QCD. The events are reweighted as, 


$$
W(y)=\frac{(d \sigma / d y)_{\mathrm{HNNLO}}}{(d \sigma / d y)_{\mathrm{HJ}-\mathrm{MiNLO}}}
$$

which brings the inclusive Higgs boson kinematics and cross sections from POWHEG in agreement with several other NNLO predictions. In practice, the resultant events can also be reweighted to the best available cross section and branching ratio predictions.

\subsubsection{NLO merging via the FxFx process in aMC@NLO}

Another important aspect of modelling the gluon fusion process is the representation of final states containing multiple jets, particularly as it pertains to modelling ggF signal "contamination" in search regions meant to isolate VBF and VH signal. Typically, the highest order of a ggF calculation is only valid for inclusive final states, e.g. the Powheg NNLOPS approach has NNLO accuracy for $\sigma_{\geq 0 j}$, NLO accuracy for $\sigma_{\geq 1 j}$, and LO accuracy for $\sigma_{\geq 2 j}$. However, it is possible for some MC generators to produce NLO accurate calculations across several multi-jet final states through the use of so-called NLO merging techniques.

The FxFx technique, as implemented in the MADGraPh5_aMC@NLO MC generator, is one such method to generate NLO-merged predictions, producing samples of ggF signal containing 0,1 , and $\geq 2$-jet final states, all calculated at NLO QCD accuracy [92]. In the FxFx approach, events are first generated at parton-level with 0, 1 , and $\geq 2$-jet final states, then overlapping parts of the phase space are "subtracted" using the SMC (currently only supported by PYTHIA8). In order to remove the overlapping components, a requirement is made that all hard scatter jets with transverse momentum above a merging scale, $\mu_{q}$, match jets generated from the parton shower generator within a minimum, user-defined $\Delta R=\sqrt{\Delta \eta^{2}+\Delta \phi^{2}}$ (typically $\Delta R<1.0$ ). 
The value of the merging scale $\mu_{q}$ tends to be set near to the jet $p_{\mathrm{T}}$ threshold in a given analysis, which for ATLAS analyses is typically $30 \mathrm{GeV}$ (see Section 6.2 for more details). This choice is motivated by the fact that SMC generators excel at modelling low energy (or soft) radiation, while parton-level MC generators excel at modelling high energy (or hard) radiation. That is to say, if $\mu_{q}$ is too high, the parton shower program effectively models all jet radiation, negating the improvements brought by using an NLO calculation. Conversely, if $\mu_{q}$ is set too low, all jet modelling will be done by the parton-level generator, so the benefits of the SMC generator are compromised. The effects of the merging scale on the Higgs boson and jet kinematics were found to be mostly negligible for a range of $\mu_{q}$ between 30 and $50 \mathrm{GeV}$, as shown in Appendix C. Therefore, the merging scale was (somewhat arbitrarily) set to a central value of $\mu_{q}=40 \mathrm{GeV}$.

Currently, MAdGraph5_aMC@NLO is unable to model interactions featuring both Higgs boson production at NLO QCD and a Higgs boson decay chain generated via MADSPIN, a consquence of the manner in which initial/final state spin correlations are handled in the generator. However, such an approach is desirable when simulating the ggF-mediated $H \rightarrow Z Z^{*} \rightarrow 4 \ell$ process under the presence of anomalous Higgs boson couplings affecting the $H Z Z$ vertex (detailed further in Section 5.3). To remedy this issue, one can invoke the decay modelling program MADSPIN using the option set spinmode none. The use of this option allows MADSPIN to neglect spin correlations between the production and decay vertices (which can be ignored a priori for a spin0 Higgs boson), facilitating multi-particle and off-shell particle decays, thus allowing the $H \rightarrow Z Z^{*} \rightarrow 4 \ell$ process to be generated.

In the MADGRAPH+ MADSPIN procedure, $H+$ jets events are generated at 


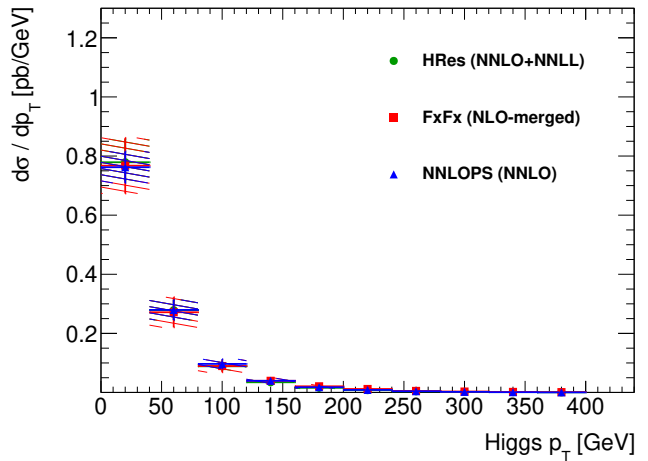

(a) Inclusive

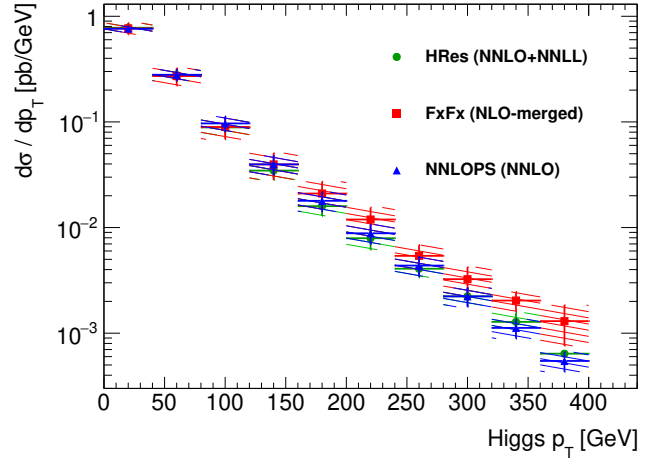

(b) Inclusive (log scale)

Figure 5.1: Comparison of the inclusive Higgs $p_{\mathrm{T}}$ spectrum of various generators: HRes, Powheg Nnlops, and MadGraph5_aMC@NLO FxFx, with each weighted to the best available $\left(\mathrm{N}^{3} \mathrm{LO}\right)$ cross section prediction.

NLO with an un-decayed Higgs boson, and the resultant Les Houches Event (LHE) record is read back into MADGRAPH5_aMC@NLO automatically. The $4 \ell$ decay is then generated by MADSPIN for each event, conserving the energy/momentum of the Higgs boson. The final event record is saved in a new LHE file, which now contains the full $g g \rightarrow H\left(\rightarrow Z Z^{*} \rightarrow 4 \ell\right)+$ jets production and decay chain. Furthermore, if the model being used contains new, anomalous Higgs boson couplings, then this process allows events to be generated at NLO QCD with anomalous couplings in both the production and decay vertices.

A comparison of the inclusive Higgs boson $p_{\mathrm{T}}$ distribution is given in Figure 5.1 for various "state-of-the-art" gluon fusion MC generators - namely, Powheg NnLOPS, HRes, and MadGraph5_aMC@NLO FxFx generators. The HRes generator, in particular, produces inclusive (but not per-event) predictions at NNLO, making its predictions close to the latest possible estimates of the ggF-mediated Higgs boson $p_{\mathrm{T}}$ spectrum. Good agreement is seen between the NNLOPS distribution with HRES. After reweighting the cross section of the $0 j$ component of the FxFx sample to match 
the NNLO prediction, the differential $p_{\mathrm{T}}$ spectrum of the FxFx sample also agrees fairly well with the spectra of the other generators. However, there is a notable divergence in the FxFx distribution at high $p_{\mathrm{T}}^{H}$, which can be explained by the fact that the model used to generate the MADGRAPH prediction uses the heavy top approximation to perform NLO QCD calculations, while the Powheg NnLOPS and HREs generators incorporate quark mass effects to NLO QCD [93, 94].

Beyond inclusive predictions, one must also consider how well a given $\mathrm{MC}$ generator describes final states with one or more jets, which is particularly critical when studying production modes such as VBF, which produce multi-jet final states. In particular, for the $H+2 j$ final state, ggF signal can "contaminate" the VBF phase space by upwards of $25 \%$ if cuts are not imposed to sufficiently distingish between production modes [95]. Therefore, to study final states with jets, one must employ a generator which can model such processes with sufficient accuracy. To this end, Figure 5.2 shows a comparison of the truth-level kinematics of several variables which characterize the $H+2 j$ final state, produced using different MC generators. Namely, NLO-merged results from MADGRAPH5_aMC@NLO are shown, as well as results from Powheg Nnlops, which is LO for $\geq 2$-jet predictions. The Powheg HJJ generator, which provides $H+2 j$ predictions at NLO QCD via the MiNLO approach, serves as a baseline for the other generators. The cross sectional differences between generators have been factored out by scaling each distribution to unit area.

Overall, fair agreement is observed in both the FxFx and NNLOPS samples with respect to the HJJ generator, although the Higgs and jet $p_{\mathrm{T}}$ spectra are on average softer for the NNLOPS sample. A possible reason for this discrepancy is the fact that any third jet in the NNLOPS sample is necessarily modelled by the SMC, rather 

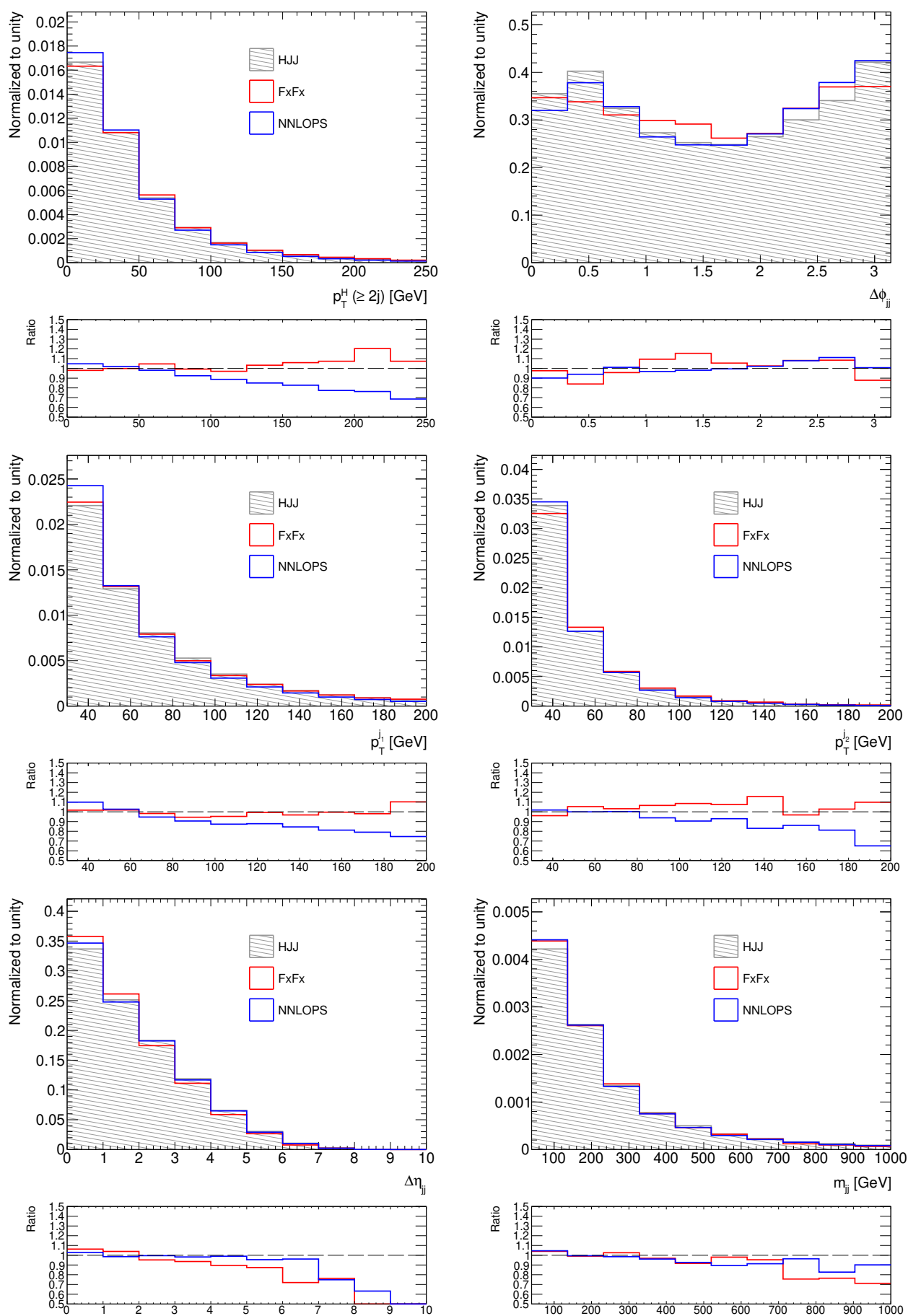

Figure 5.2: Kinematic shapes of important variables in $\geq 2$-jet ggF final states for different MC generators, with each distribution scaled to unit area. 
than the HS generator. Accordingly, the components of the $p_{\mathrm{T}}$ spectra which would correspond to a "hard" third jet with high $p_{\mathrm{T}}$ are likely not well-modelled, leading the overall $p_{\mathrm{T}}$ spectra of the Higgs and jets to be somewhat softer. However, as the cross sectional uncertainty for $\operatorname{ggF} H+2 j$ predictions at $\mathrm{LO}$ is $\mathcal{O}(50 \%)$, such deviations are not totally unexpected between LO and NLO generators. The agreement in dijet variables such as $m_{\mathrm{jj}}$ and $\Delta \eta_{\mathrm{jj}}$ tends to fare better between generators, as such variables are not strongly influenced by the presence of a third jet, except in the extrema of the distributions. The FxFx and HJJ samples tend to agree well across the full range of variables tested, as both generate $H+2 j$ predictions at NLO QCD, providing a good justification for the use of the FxFx merging approach to model ggF production with multiple final state jets.

\subsection{Vector boson fusion, associated production}

The vector boson fusion and all-hadronic $W / Z$ associated production processes are intrinsically linked, in that they both proceed through weak boson-mediated $q_{1} q_{2} \rightarrow \mathrm{H}+$ $q_{3} q_{4}$ diagrams. So, in practice, these two processes can interfere with each other, introducing a small effect on the combined cross section of the two production modes. Functionally speaking, due to the low number of signal events expected in the $H \rightarrow 4 \ell$ channel, the visibility of the interference is diminished, so it is possible to treat the processes separately with no appreciable loss of accuracy.

Due to the relatively small effects of higher-order QCD corrections for the VBF and hadronic VH processes, the kinematics of each process are generally well-described at tree-level, as shown in Figures 5.3 and 5.4. Here, the differential distributions of several key variables computed at $\mathrm{LO}$ are compared to the equivalent distributions at 
NLO QCD, with the latter being reweighted using a $k$-factor to bring the inclusive cross section to NNLO QCD. Such a reweighting procedure is standard for Monte Carlo generation within ATLAS, since oftentimes "state-of-the-art" predictions entail only numerical cross section calculations, rather than actual MC event generation.

The dominant effect of the higher-order corrections is evidently a scaling of the total cross section for both VBF and VH production. That is to say, one observes that the ratios of the LO and NNLO-reweighted distributions tend to be offset by a mostly constant factor, indicating only minor changes between the process kinematics at LO and NLO. A notable exception is seen for the dijet pseudorapidity separation $\Delta \eta_{\mathrm{jj}}=$ $\left|\eta_{j_{1}}-\eta_{j_{2}}\right|$ in VBF production, as the distribution evidently shifts rightward at NLO. However, depending on the region of phase space one is interested to study, and the experimental cuts which are applied, the behaviour of the higher-order processes may be reasonably well-approximated using a simple $k$-factor applied to the LO prediction.

\subsection{The Higgs Characterization model}

A large focus of current ATLAS Higgs analyses is to probe for BSM (or generally non-SM) behaviour in the various Higgs production modes. One method to probe for potential contributions of BSM physics is through the use of an effective field theory (EFT). The fundamental concept behind an EFT is that the Standard Model Lagrangian can be perturbed by new, non-SM fields in such a way that $\mathcal{L}=\mathcal{L}_{\mathrm{SM}}+\Delta \mathcal{L}$. The non-SM contributions tend to be suppressed by a newly-defined energy scale $\Lambda$, above which new, strongly-coupled physics is proposed to exist. Below this threshold, any new couplings with Standard Model particles are suppressed, meaning that they can be treated in the context of perturbation theory. 

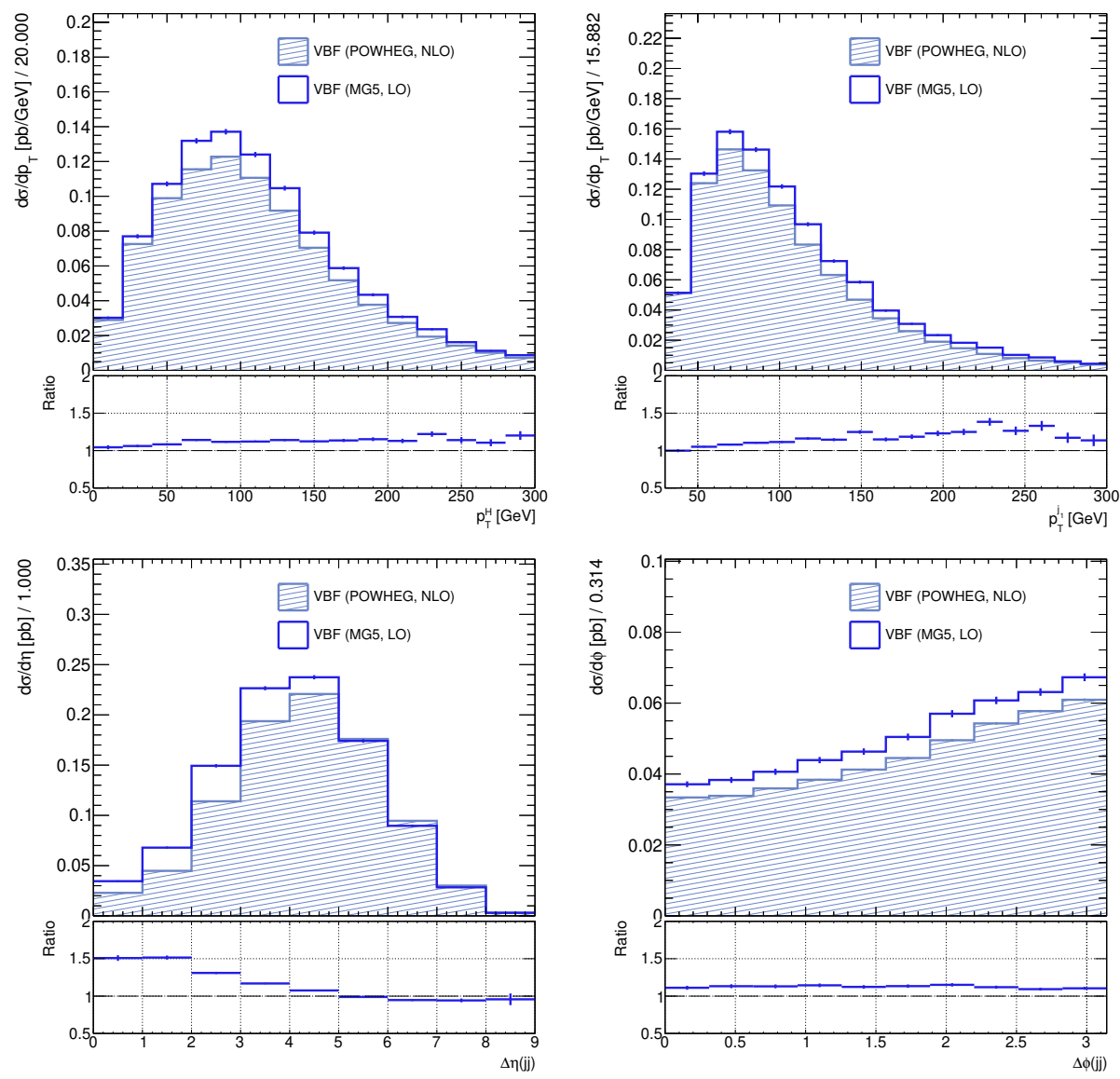

Figure 5.3: Comparison of the LO and NLO QCD differential cross section distributions for VBF Higgs boson production at $\sqrt{s}=13 \mathrm{TeV}$. The inclusive cross section of the NLO QCD component has been reweighted to match the best-known NNLO QCD cross section. 

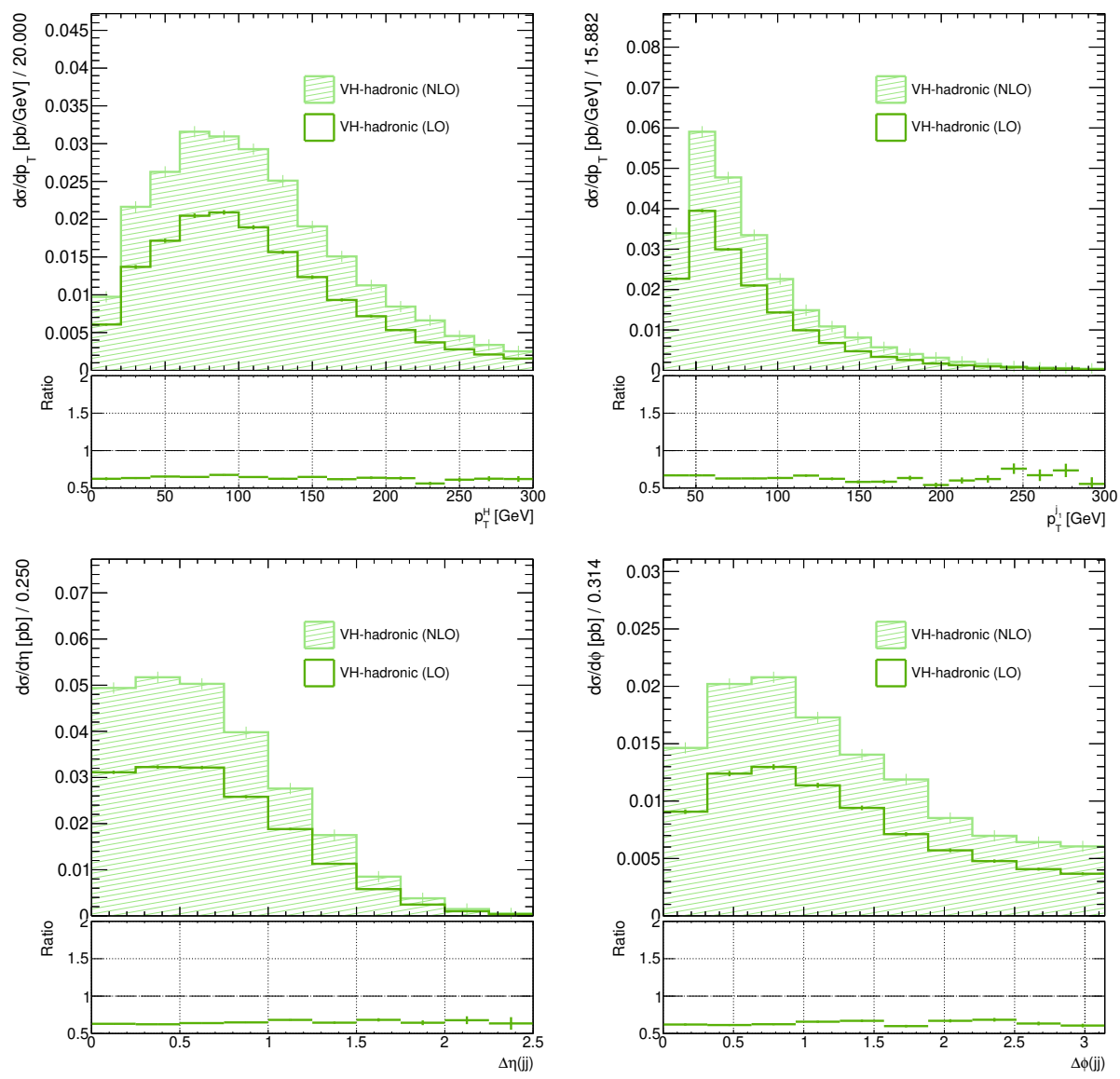

Figure 5.4: Comparison of the LO and NLO QCD differential cross section distributions for hadronic VH Higgs boson production at $\sqrt{s}=13 \mathrm{TeV}$. The inclusive cross section of the NLO QCD component has been reweighted to match the best-known NNLO QCD cross section. 
One such EFT is the Higgs Characterization (HC) model [96], which implements several new operators that correspond to the lowest-dimensional descriptions of interactions common to many BSM models. In particular, the couplings between the Higgs boson and the $W^{ \pm}$and $Z$ bosons, photons, and gluons can all be modified by the presence of new, BSM interactions. The model further assumes that all interactions are the result of couplings to a single spin-0 boson, with the non-SM interactions supressed by a factor $1 / \Lambda$. The cutoff scale is typically set to $\Lambda=1 \mathrm{TeV}$, as no evidence of new physics has been observed at lower energy scales at the LHC, or at previous collider experiments.

The Higgs boson in this model can have both $\mathcal{C P}$-even and $\mathcal{C P}$-odd properties, controlled by a mixing angle $\alpha$, allowing for interactions which are purely $\mathcal{C P}$-even or odd, or of a mixed-CP state. The model is implemented in MADGraph5_aMC@NLO [96, 97], with NLO QCD precision possible in the Higgs boson production vertices, and the $H \rightarrow Z Z^{*} \rightarrow 4 \ell$ decay computed at leading order. All coupling strength parameters are configurable, along with the new energy scale $\Lambda$ and $\cos \alpha$. In practice, $\cos \alpha$ tends to be set to $1 / \sqrt{2}$ to maximize the degree of mixing when $\mathcal{C P}$-odd couplings are introduced.

The Higgs boson couplings to the $W^{ \pm}$and $Z$ bosons in the HC model are expressed through the interaction Lagrangian, 


$$
\begin{aligned}
& \mathcal{L}_{0}^{V}=\left\{c_{\alpha} \kappa_{\mathrm{SM}}\left[\frac{1}{2} g_{H Z Z} Z_{\mu} Z^{\mu}+g_{H W W} W_{\mu}^{+} W^{-\mu}\right]\right. \\
&-\frac{1}{4} \frac{1}{\Lambda}\left[c_{\alpha} \kappa_{H Z Z} Z_{\mu \nu} Z^{\mu \nu}+s_{\alpha} \kappa_{A Z Z} Z_{\mu \nu} \tilde{Z}^{\mu \nu}\right] \\
&-\frac{1}{2} \frac{1}{\Lambda}\left[c_{\alpha} \kappa_{H W W} W_{\mu \nu}^{+} W^{-\mu \nu}+s_{\alpha} \kappa_{A W W} W_{\mu \nu}^{+} \tilde{W}^{-\mu \nu}\right] \\
&\left.-\frac{1}{\Lambda} c_{\alpha}\left[\kappa_{H \partial Z} Z_{\nu} \partial_{\mu} Z^{\mu \nu}+\kappa_{H \partial W} W_{\nu}^{+} \partial_{\mu} W^{-\mu \nu}+(\text { h.c. })\right]\right\} \mathcal{X}_{0}
\end{aligned}
$$

In this expression, $\mathcal{X}_{0}$ represents the Higgs field, $V^{\mu}$ represents the weak boson fields $\left(V=Z, W^{ \pm}\right)$, with the $V^{\mu \nu}$ terms corresponding to higher-dimensional field tensors, such that $\tilde{V}^{\mu \nu}=\frac{1}{2} \varepsilon^{\mu \nu \rho \sigma} V_{\rho \sigma}$. The $g_{H V V}$ are dimensionful couplings tuned to reproduce Standard Model behaviour when all anomalous coupling terms are zero. To distinguish the $\mathcal{C P}$-even and $\mathcal{C P}$-odd contributions to the Lagrangian, the following notation is adopted as convention,

$$
s_{\alpha}=\sin \alpha, \quad c_{\alpha}=\cos \alpha
$$

The symbols $\kappa_{\mathrm{SM}}, \kappa_{H V V}$, and $\kappa_{A V V}$ denote coupling strength factors corresponding to Standard Model, BSM $\mathcal{C} \mathcal{P}$-even, and BSM $\mathcal{C} \mathcal{P}$-odd interactions with the weak bosons, respectively. To ensure that the Lagrangian terms are Hermitian, all coupling strength factors $\kappa$ are assumed to be real. The terms $\kappa_{H \partial Z}$ and $\kappa_{H \partial W}$ correspond to coupling strength factors for higher-order derivative operators, which can have some effect on the on-shell and off-shell $Z$ boson masses in $H \rightarrow Z Z^{*} \rightarrow 4 \ell$ decays, but are otherwise not of immediate interest. Operators corresponding to $Z-\gamma$ interactions also exist in this model, but they have been neglected in the above Lagrangian, as they do not impact the interactions studied in the $H \rightarrow Z Z^{*} \rightarrow 4 \ell$ channel. 
A comparison of the truth-level distributions of a number of BSM-sensitive variables are shown for LO VBF + VH Higgs production in Figure 5.5. The distributions are presented under three scenarios: the SM case of $\kappa_{\mathrm{SM}}=1$ only; a pure $\mathcal{C} \mathcal{P}$-odd Higgs boson with $\kappa_{A V V}=1$ only; and a pure BSM $\mathcal{C} \mathcal{P}$-even Higgs boson which has $\kappa_{H V V}=1$ only. The distributions are shown normalized to unity to emphasize the differences in shape expected under each BSM scenario. For example, the Higgs boson and leading jet $p_{\mathrm{T}}$ uniformly increase in the BSM scenarios, leading to a relative increase in the final state jet multiplicity. Conversely, the dijet mass and pseudorapidity separation of the jets decrease, indicating that the forward jets characteristic of VBF become much more central in each BSM scenario.

Analogous to the Lagrangian structure of the $W^{ \pm}$and $Z$ boson interactions, the Higgs boson can couple to pairs of gluons through $\mathcal{C P}$-even and odd interactions, mediated by the mixing angle $\alpha$. Accordingly, $\mathcal{C P}$-mixed states and $\mathcal{C P}$-violation are also possible in gluon fusion-mediated Higgs boson production. The Higgs bosongluon interaction vertices are described by the effective Lagrangian,

$$
\mathcal{L}_{0}^{g}=-\frac{1}{4}\left[c_{\alpha} \kappa_{H g g} g_{H g g} G_{\mu \nu}^{a} G^{a, \mu \nu}+s_{\alpha} \kappa_{A g g} g_{A g g} G_{\mu \nu}^{a} \tilde{G}^{a, \mu \nu}\right] \mathcal{X}_{0}
$$

where $g_{H g g}$ is again tuned to reproduce SM conditions when no anomalous couplings are present, and the field strength tensor $G_{\mu \nu}^{a}$ is defined as,

$$
G_{\mu \nu}^{a}=\partial_{\mu} G_{\nu}^{a}-\partial_{\nu} G_{\mu}^{a}+g_{s} f^{a b c} G_{\mu}^{b} G_{\mu}^{c}
$$

It it worth noting that this model implements a "Higgs effective field theory" approach to modelling ggF production, meaning that the heavy top limit is used to 

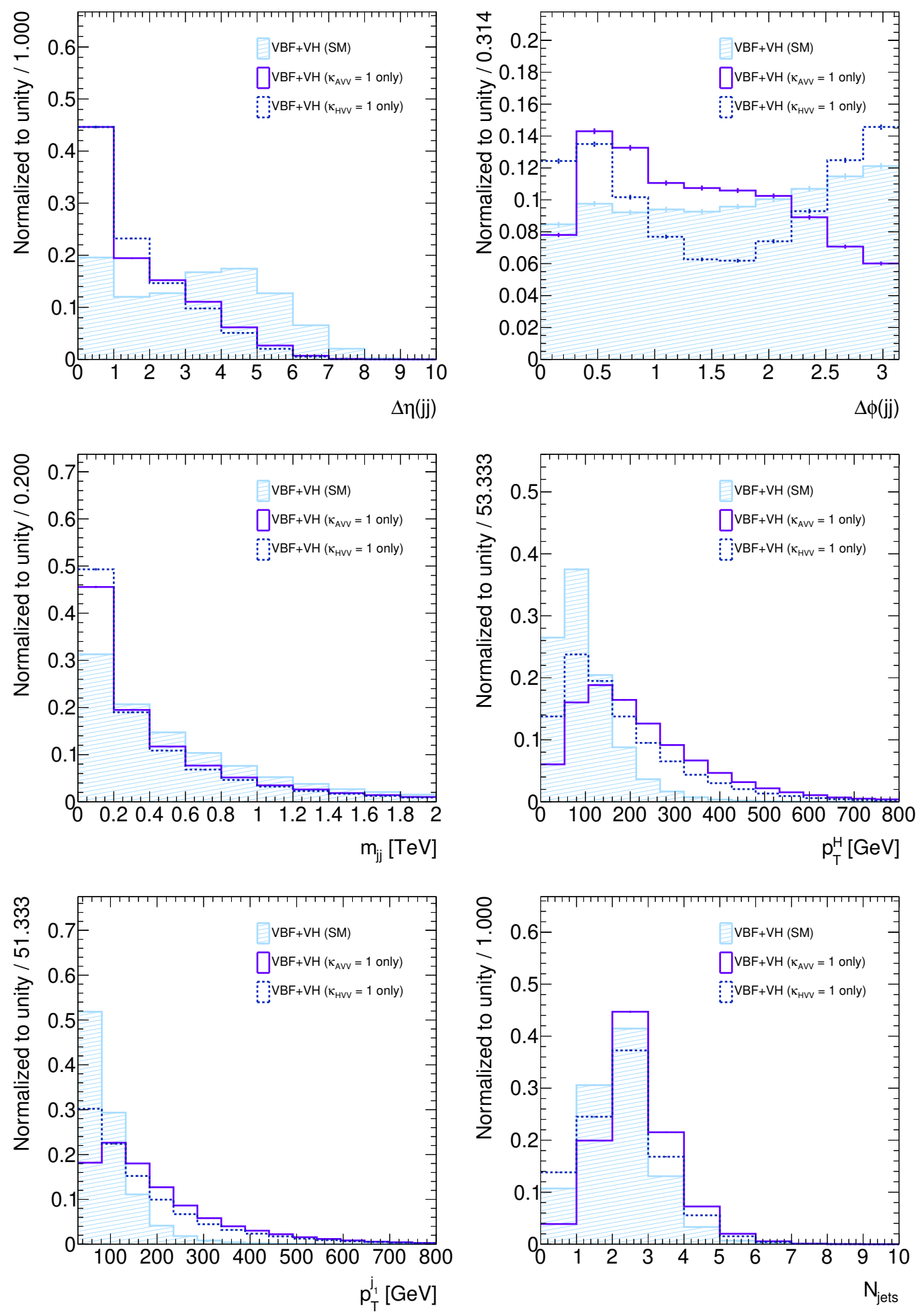

Figure 5.5: Comparison of the kinematics in LO VBF + VH Higgs production under different coupling scenarios using the Higgs Characterization model. 
reduce the Higgs-gluon interaction vertex to an effective contact interaction. Here, the first term in the effective Lagrangian of Equation 5.3 represents the Standard Model $\mathcal{C P}$-even gluon fusion vertex, while the second term contains the $\mathcal{C P}$-odd operator.

Truth-level distributions for a number of BSM-sensitive variables are shown for NLO-merged ggF Higgs production in Figure 5.6. The distributions are presented for the nominal case of $\kappa_{H g g}=1$, and for the pure $\mathcal{C} \mathcal{P}$-odd case of $\kappa_{A g g}=1$ only. The distributions are shown normalized to unity to emphasize any differences in shape under the BSM scenario. Unlike the stark differences in kinematics observed in VBF + VH Higgs production under different BSM scenarios, the behaviour of SM and $\mathcal{C P}$-odd gluon fusion is largely the same (within the MC statistical uncertainty), with the exception of the variable $\Delta \phi_{j j}$, which peaks visibly more towards $\pi / 2$ for $\mathcal{C P}$ odd Higgs boson production. Therefore, the presence of $\mathcal{C P}$-odd interactions in the ggF production vertex would practically only be indicated by deviations in the total measured signal yield from the SM expectation, with further constraints possible based on the shape of $\Delta \phi_{j j}$.

Generally speaking, the BSM terms described in Equations 5.2 and 5.3 are expected to change the relative contributions of the $\mathrm{VBF}$ and $\mathrm{VH}$ processes with respect to $\mathrm{ggF}$, which is the dominant production mode at the LHC for a Standard Model Higgs boson. For large values of the $H V V$ BSM couplings, the $\mathrm{VBF}+\mathrm{VH}$ contribution can have a total cross section which is comparable to, or larger than, the SM ggF process. Likewise, the presence of a non-zero BSM $\mathcal{C P}$-odd coupling scale factor $\kappa_{A g g}$ can also change the gluon fusion cross section with respect to the SM prediction. These effects manifest in different ways; as shown in the left plot of Figure 5.7, the cross sections for the ggF and $\mathrm{VBF}+\mathrm{VH}$ processes increase quadratically as a func- 

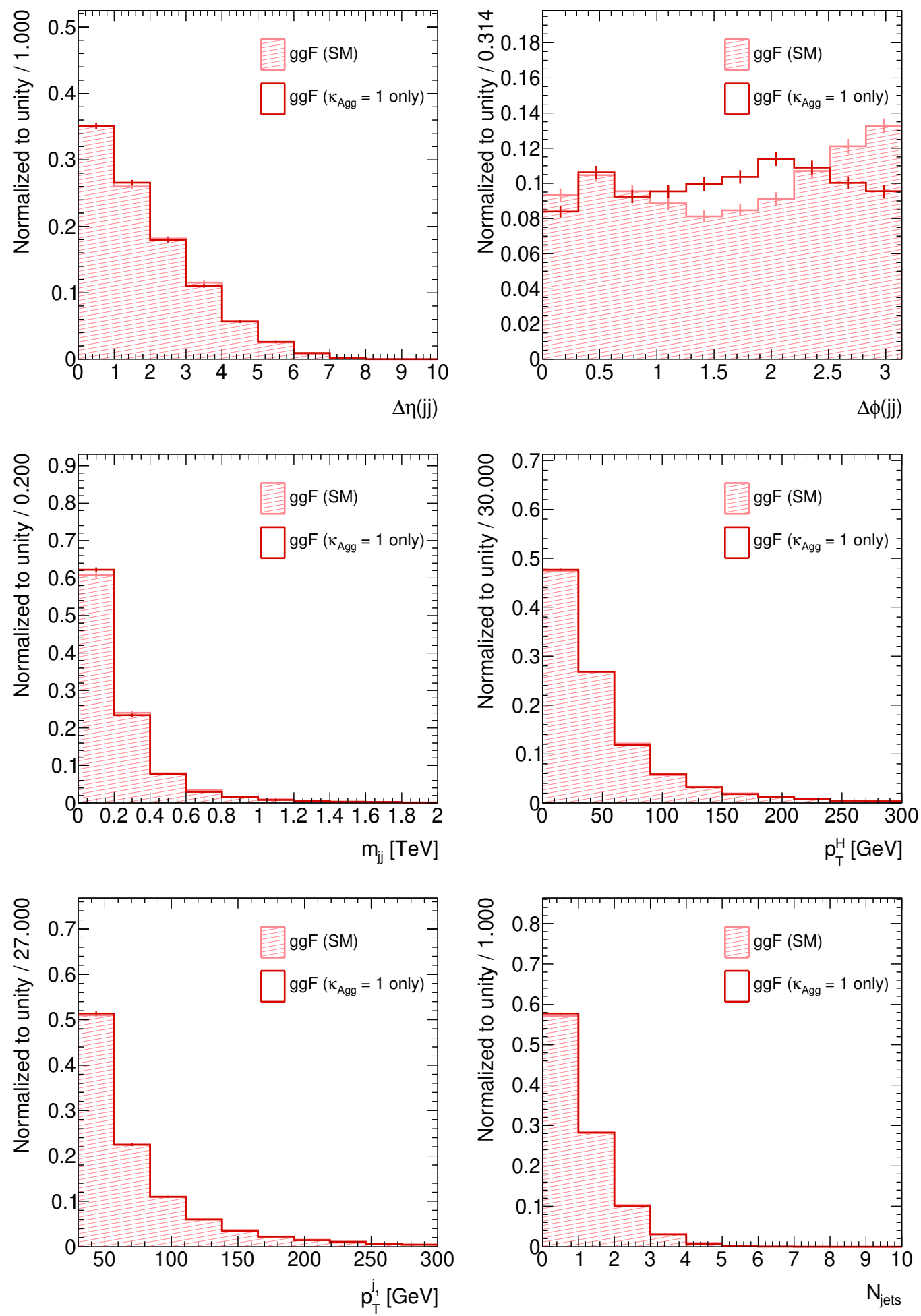

Figure 5.6: Comparison of the kinematics in NLO-merged ggF Higgs production for $\mathcal{C} \mathcal{P}$-even and $\mathcal{C P}$-odd coupling scenarios using the Higgs Characterization model. 

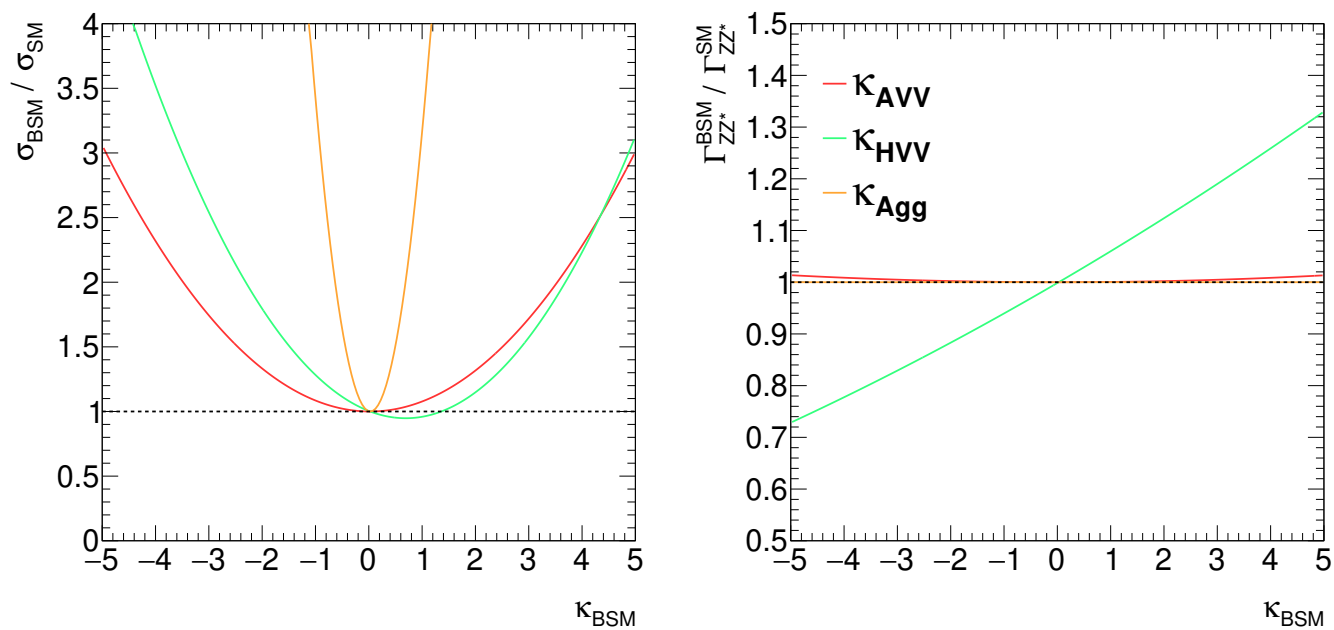

Figure 5.7: Changes to the Higgs production cross sections (left) and $Z Z^{*}$ branching ratio (right) as a function of different anomalous coupling strength constants in the Higgs Characterization model.

tion of the different anomalous coupling strength constants, while in the right plot, the $Z Z^{*}$ partial width can undershoot or overshoot SM expectations, depending on the sign and magnitude of the associated coupling strength. In particular, interference effects are visible when the BSM $\mathcal{C} \mathcal{P}$-even coupling is present, as mediated by the constant $\kappa_{H V V}$, leading to an appreciable decrease in $\Gamma_{Z Z^{*}}$ for negative values of $\kappa_{H V V}$. For the VBF+VH processes, these interactions lead to changes in the matrix element calculation which are $\mathcal{O}\left(\left(\kappa_{\mathrm{BSM}}\right)^{4}\right)$, hence $\sigma \times \mathcal{B}$ changes as $\left(\kappa_{\mathrm{BSM}}\right)^{4}$ for each.

\subsubsection{Higgs total width modifications}

When introducing new, anomalous Higgs couplings, not only may process cross sections and partial widths change, but the total width of the Higgs boson $\left(\Gamma_{H}\right)$ may change, as well. Since the SM branching ratios for the $H \rightarrow W W^{*}$ and $H \rightarrow Z Z^{*}$ decays are well-known for $m_{H}=125 \mathrm{GeV}$ as $\Gamma_{W W^{*}} / \Gamma_{H}=0.214$ and $\Gamma_{Z Z^{*}} / \Gamma_{H}=0.026$ [41], so the change in the Higgs boson total width under the pres- 


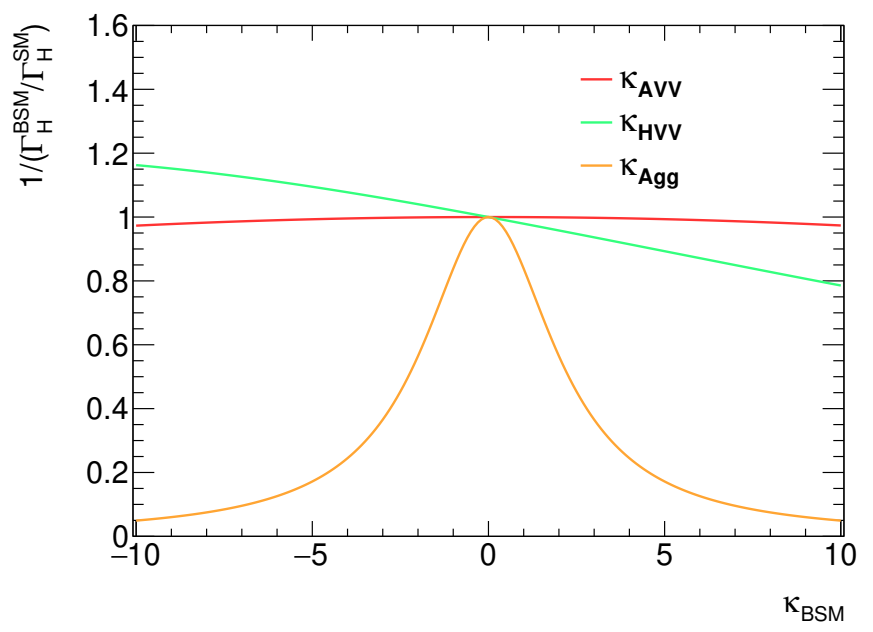

Figure 5.8: The inverse of the change in the Higgs boson total width $\Gamma_{H}$ as a function of different anomalous couplings in the Higgs Characterization model, which serves as a correction factor to the branching ratios predicted by the $\mathrm{HC}$ model.

ence of anomalous $H V V$ couplings can be expressed as,

$$
\frac{\Gamma_{H}^{\mathrm{BSM}}}{\Gamma_{H}^{\mathrm{SM}}}=0.760+0.214 \times f_{\kappa_{x W W}}^{\mathcal{B}}+0.026 \times f_{\kappa_{x Z Z}}^{\mathcal{B}} .
$$

Likewise, under the presence of a modified ggF coupling such as $\kappa_{A g g}$, the change in the total width becomes,

$$
\frac{\Gamma_{H}^{\mathrm{BSM}}}{\Gamma_{H}^{\mathrm{SM}}}=0.9143+0.0857 \times f_{\kappa_{A g g}}^{\mathcal{B}} .
$$

These total width modifications are not implemented directly in the $\mathrm{HC}$ model, so they are instead computed by performing polynomial fits to the relevant Higgs boson branching ratios as a function of the different anomalous couplings, which allows one to obtain $f_{\mathcal{K}_{x W W}}^{\mathcal{B}}$ and $f_{\kappa_{x Z Z}}^{\mathcal{B}}$, and scale $\Gamma_{H}$ accordingly. That is to say, applying the inverse of $\frac{\Gamma_{H}^{\mathrm{BSM}}}{\Gamma_{H}^{\mathrm{SM}}}$ to the predicted branching ratio allows for an estimate of $\mathcal{B}\left(Z Z^{*}\right)$ which correctly factors in the change in total width. 
In Figure 5.8, the inverse of the change in $\Gamma_{H}$ is shown as a function of different anomalous couplings present in the $\mathrm{HC}$ model, which serves as the correction factor to $\mathcal{B}\left(Z Z^{*}\right)$. While the change in total width under $\kappa_{A V V}$ and $\kappa_{H V V}$ is within $20 \%$ for large values of each coupling, the presence of even a small value of $\kappa_{A g g}$ can induce a strong modification of $\Gamma_{H}$. Therefore, for extremely large values of $\kappa_{A g g}$, the value of $\sigma_{\mathrm{ggF}} \times \mathcal{B}\left(Z Z^{*}\right)$ predicted by the $\mathrm{MC}$ can be overestimated by upwards of $90 \%$ if the change in $\Gamma_{H}$ is not considered.

\subsection{Histogram morphing}

When introducing anomalous couplings through an EFT for the purposes of constraining BSM phenomena, it is imperative that the new physics under study can be adequately and efficiently probed, particularly when dealing with a potentially infinite parameter space of real-valued coupling strengths. In the following chapters, the histogram morphing technique $[98,99,100]$ is used to model changes to event yields and initial/final state kinematics in a continuous manner within a multidimensional space of coupling parameters $\vec{g}=\left\{g_{1}, \ldots, g_{n}\right\}$. The method is analogous to an eigenvector-eigenvalue problem, in that one seeks to represent arbitrary points in the coupling parameter space using a morphing basis composed of $N$ base samples, $T_{\mathrm{in}}^{1}, \ldots, T_{\mathrm{in}}^{N}$, with known couplings. This method applies to processes for which the matrix element squared $|\mathcal{M}(\vec{g})|^{2}$ can be factorized in terms of the coupling strengths $g_{i}$, and the corresponding probability amplitudes $\mathcal{O}_{i}$, such that [99], 


$$
|\mathcal{M}(\vec{g})|^{2}=\underbrace{\left(\sum_{i=1}^{n_{p}+n_{s}} g_{i} \mathcal{O}_{i}\right)^{2}}_{\text {production vertex }} \cdot \underbrace{\left(\sum_{j=1}^{n_{d}+n_{s}} g_{j} \mathcal{O}_{j}\right)^{2}}_{\text {decay vertex }}
$$

where the subscripts $p, d$, and $s$ correspond to couplings appearing in the production vertices, decay vertices, and both production and decay vertices, respectively.

In order for the morphed predictions to vary smoothly between adjacent base samples, each base sample must be normalised to its proper cross section, and weighted with a polynomial $w\left(\vec{g}_{\text {out }} ; \vec{g}_{\text {in }}^{j}\right)$ which is defined in terms of the contributing couplings. Accordingly, an arbitrary morphed output $T_{\text {out }}$ is expressed using a summation of terms containing: the squared matrix elements of the base samples, typically represented by some observable of interest $T_{\text {in }}$, such as a kinematic distribution; and coefficients that are a function of both the desired couplings, as well as those of the base samples. Or, more generically [99],

$$
T_{\text {out }}\left(\vec{g}_{\text {out }}\right)=\sum_{j=1}^{N_{\text {base }}} w_{j}\left(\vec{g}_{\text {out }} ; \vec{g}_{j}\right) \cdot T_{\text {in }}^{j}\left(\vec{g}_{j}\right) .
$$

For example, in the simplest case of a process with one BSM coupling $g_{\mathrm{BSM}}$, and one SM coupling $g_{\mathrm{SM}}$, the squared matrix element can be expressed as [99],

$$
\left|\mathcal{M}\left(g_{\mathrm{SM}}, g_{\mathrm{BSM}}\right)\right|^{2}=g_{\mathrm{SM}}^{2}\left|\mathcal{O}_{\mathrm{SM}}\right|^{2}+g_{\mathrm{BSM}}^{2}\left|\mathcal{O}_{\mathrm{BSM}}\right|^{2}+2 g_{\mathrm{SM}} g_{\mathrm{BSM}} \mathcal{R}\left(\mathcal{O}_{\mathrm{SM}}^{*} \mathcal{O}_{\mathrm{BSM}}\right)
$$

In this construction, only three samples would be needed to form a morphing basis: one sample from SM predictions, $T_{\text {in }}(1,0)$; one sample with a pure BSM prediction, 
$T_{\text {in }}(0,1)$; and a "mixed" sample which has interference between the SM and BSM states, $T_{\mathrm{in}}(1,1)$. Since a given sample $T\left(g_{\mathrm{SM}}, g_{\mathrm{BSM}}\right)$ is derived from the corresponding matrix element squared $\left|\mathcal{M}\left(g_{\mathrm{SM}}, g_{\mathrm{BSM}}\right)\right|^{2}$, in general, one can write,

$$
\begin{aligned}
& T_{\mathrm{in}}\left(g_{\mathrm{SM}, j}, g_{\mathrm{BSM}, j}\right) \propto g_{\mathrm{SM}, j}^{2}\left|\mathcal{O}_{\mathrm{SM}}\right|^{2}+ \\
& \qquad g_{\mathrm{BSM}, j}^{2}\left|\mathcal{O}_{\mathrm{BSM}}\right|^{2}+2 g_{\mathrm{SM}, j} g_{\mathrm{BSM}, j} \mathcal{R}\left(\mathcal{O}_{\mathrm{SM}}^{*} \mathcal{O}_{\mathrm{BSM}}\right) .
\end{aligned}
$$

such that the final, morphed sample $T_{\text {out }}\left(g_{\mathrm{SM}}, g_{\mathrm{BSM}}\right)$ can be expressed generically as a linear sum of the input samples as [99],

$$
T_{\text {out }}\left(g_{\mathrm{SM}}, g_{\mathrm{BSM}}\right)=\sum_{j=1}^{3}\left(a_{j, 1} g_{\mathrm{SM}}^{2}+a_{j, 2} g_{\mathrm{BSM}}^{2}+a_{j, 3} g_{\mathrm{SM}} g_{\mathrm{BSM}}\right) T_{\mathrm{in}}\left(g_{\mathrm{SM}, j}, g_{\mathrm{BSM}, j}\right) .
$$

In order to solve for the unknown $a_{j, k}$ terms, one imposes the boundary condition that $T_{\text {out }}=T_{\text {in }}^{j}$ when morphing to the $j^{\text {th }}$ base sample. Consequently, for a distribution at an arbitrary coupling point $T_{\text {out }}$, the weight of each base sample can be determined by inverting the matrix $\left[a_{j k}\right]$ which emerges from the boundary condition requirements, and applying the inverted matrix $\left[a_{j k}\right]^{-1}$ to $\left(g_{\mathrm{SM}}, g_{\mathrm{BSM}}, g_{\mathrm{SM}} g_{\mathrm{BSM}}\right)^{T}$, or the vector of coupling coefficients desired for $T_{\text {out }}[98]$.

The number of base samples required to perform the morphing is dependent on the number of couplings in the production and decay vertices. For the VBF and VH-mediated $H \rightarrow Z Z^{*} \rightarrow 4 \ell$ processes, in particular, the number of required base samples $N_{\text {base }}$ is given as [100], 


$$
N_{\text {base }}^{\mathrm{VBF}+\mathrm{VH}}=\frac{n_{1}\left(n_{1}+1\right)}{2}\left[\frac{\left(n_{1}+2\right)\left(n_{1}+3\right)}{12}+\frac{\left(n_{1}+2\right) n_{2}}{3}+\frac{\left(n_{2}+1\right) n_{2}}{2}\right]
$$

where $n_{1}+n_{2}$ couplings are present in the production vertex, and $n_{1}$ couplings are present in the decay vertex. Here, it is required that $n_{1}$ couplings are the same in both production and decay, since both Higgs decay and production proceed through $H V V$ interaction vertices. Due to the presence of the same couplings in both production and decay, the amount of base samples required for morphing can become quite non-trivial; for example, to test for the presence of two anomalous couplings simultaneously (i.e. $n_{1}=3$ ), assuming custodial symmetry and $n_{2}=0, N_{\text {base }}^{\mathrm{VBF}+\mathrm{VH}}=15$ samples would be required to model the $\mathrm{VBF} H \rightarrow 4 \ell$ process. Likewise, a morphing basis featuring four anomalous couplings, or $n_{1}=5$, would require $N_{\text {base }}^{\mathrm{VBF}+\mathrm{VH}}=70$ samples.

For the gluon fusion-mediated $H \rightarrow Z Z^{*} \rightarrow 4 \ell$ process, the expression for $N_{\text {base }}$ is simpler [100],

$$
N_{\mathrm{base}}^{\mathrm{ggF}}=\frac{1}{4} n_{p}\left(n_{p}+1\right) n_{d}\left(n_{d}+1\right)
$$

where $n_{p}$ is the number of couplings in the production vertex, and $n_{d}$ is the number of couplings in the decay vertex. So, for example, the study of ggF production with one anomalous coupling each in production and decay would require $N_{\text {base }}^{\text {ggF }}=9$ samples to fully cover the coupling space of interest. In general, any set of $N_{\text {base }}$ samples can be used as base samples for histogram morphing, given that they form a linearly independent morphing basis. That is to say, it is required that each base sample must not be redundant, or able to be expressed as a linear combination of the other base 
samples, analogous to the eigenvector basis for a diagonalizable matrix.

Given an arbitrarily large coupling parameter space, deriving the best choice of morphing basis is a non-trivial task. If the couplings of the base samples are too close together in the parameter space, then in the extrema of each coupling, the morphed observables will be poorly represented, and vice versa for bases with couplings that are too broadly separated in the parameter space. One metric to determine if a set of base samples is adequately performant uses the effective number of events, $n_{\mathrm{eff}}$, defined as [100],

$$
n_{\mathrm{eff}}=\sum_{i=1}^{n} W_{i}(\vec{\kappa}) N_{i}\left(\vec{\kappa}_{i}\right),
$$

together with the total number of events $\left(n_{\text {tot }}\right)[100]$,

$$
n_{\mathrm{tot}}=\sum_{i=1}^{n}\left|W_{i}(\vec{\kappa})\right| N_{i}\left(\vec{\kappa}_{i}\right) .
$$

to form a ratio $n_{\mathrm{eff}} / n_{\mathrm{tot}}$, which provides a measure of how well the morphing basis represents a given coupling configuration. In particular, a low value of $n_{\text {eff }} / n_{\text {tot }}$ implies the presence of a "gap" in the basis coverage, resulting in a poor reproduction of the true underlying physics of the model at that point in the parameter space. If the morphed observable is a histogram, for example, a low value of $n_{\text {eff }} / n_{\text {tot }}$ at a given coupling point may manifest as large fluctuations in the bin-by-bin yields, relative to an equivalent histogram generated directly from Monte Carlo. Conversely, a high value of $n_{\text {eff }} / n_{\text {tot }}$ suggests that the morphed observable will be indistinguishable from one generated directly from MC.

In practice, values of $n_{\text {eff }} / n_{\text {tot }}>0.5$ tend to provide an acceptable representation, 

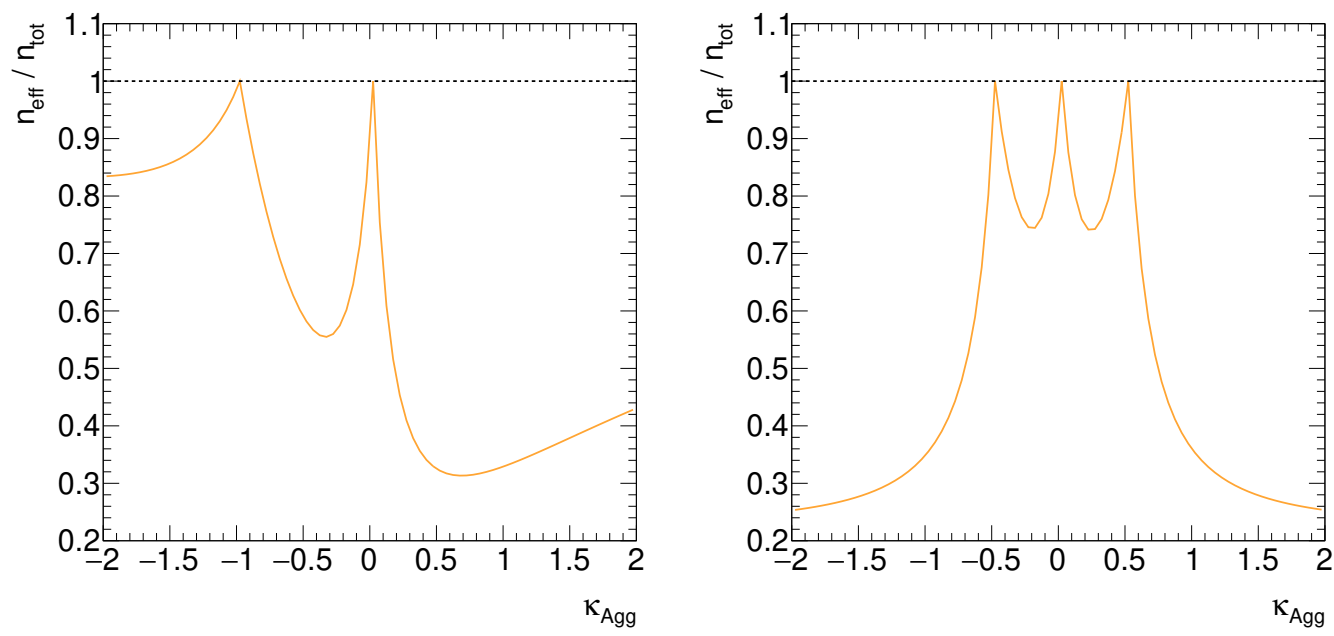

Figure 5.9: Comparison of $n_{\text {eff }} / n_{\text {tot }}$ for morphing bases composed of $\left(\kappa_{H g g}, \kappa_{A g g}\right)=(1,0),(0,1)$, and $(1,-1)$ (left), and $\kappa_{A g g}$ in $[-0.5,0,0.5]$ with $\kappa_{H g g}=1$ (right).

although $n_{\text {eff }} / n_{\text {tot }}$ should be as high as possible to minimize the effects of statistical fluctuations from gaps in basis coverage. For example, one aspect of the anomalous couplings analysis in the $H \rightarrow Z Z^{*} \rightarrow 4 \ell$ channel involves probing for $\mathcal{C P}$-odd interactions in the $\mathrm{ggF}$ production vertex (i.e. $\kappa_{A g g} \neq 0$ ). As shown in the left plot of Figure 5.7, the region between $\kappa_{A g g}= \pm 0.5$ and \pm 1 induces $\sim 50 \%$ deviations in the expected SM ggF cross section. Therefore, to place an initial constraint on the range of $\kappa_{A g g}$, one can require that the absolute upper/lower bounds of $\kappa_{A g g}$ in the morphing basis should be (somewhat arbitrarily) between \pm 0.5 and \pm 1 .

Initial tests were made using a basis formed from samples with $\left(\kappa_{H g g}, \kappa_{A g g}\right)=$ $(1,0),(0,1)$, and $(1,-1)$ to examine the viability of such a configuration. However, the resultant distributions of $n_{\text {eff }} / n_{\text {tot }}$ were found to be heavily asymmetrical, with the region of $\kappa_{\text {Agg }}$ not covered by the $\mathcal{C P}$-even/odd mixed sample having a poor representation, as shown in the left plot of Figure 5.9. Furthermore, two-dimensional distributions of $n_{\text {eff }} / n_{\text {tot }}$ in terms of $\kappa_{H g g}$ and $\kappa_{A g g}$, as shown in Figure 5.10, demonstrated 


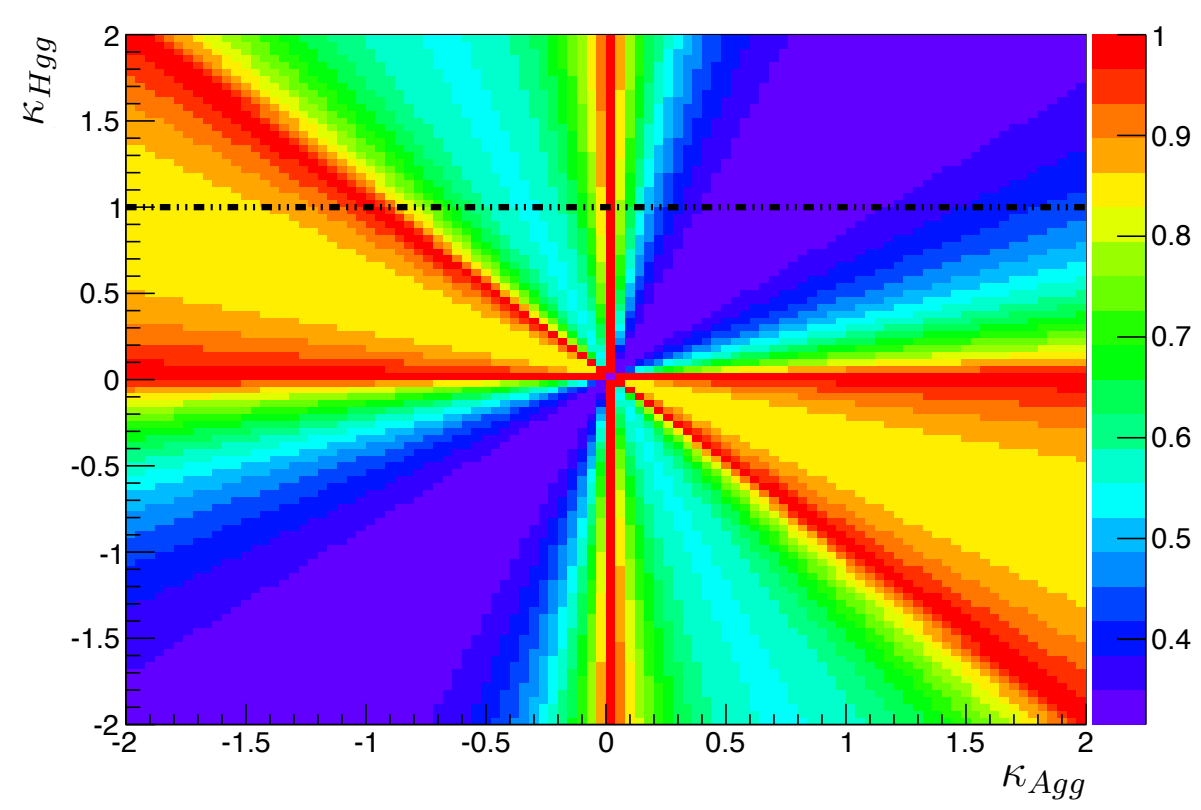

(a) $\left(\kappa_{H g g}, \kappa_{A g g}\right)=(1,0),(0,1)$, and $(1,-1)$

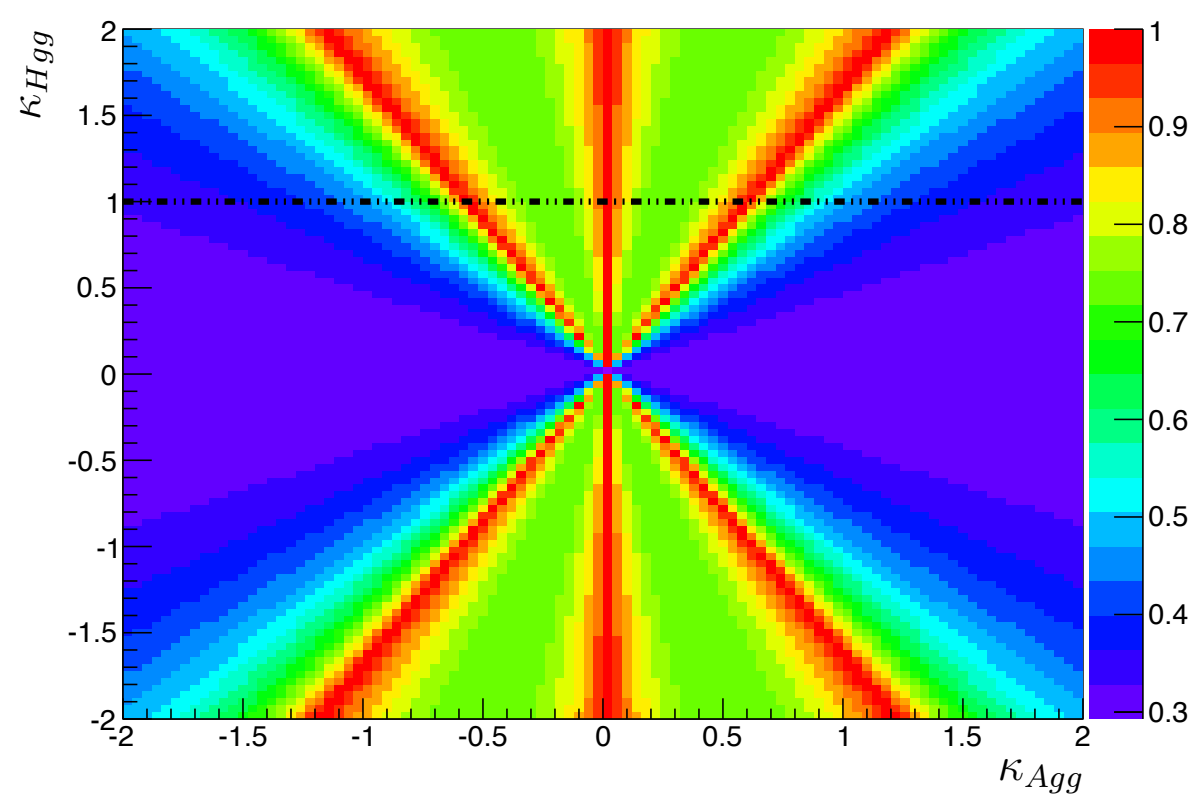

(b) $\left(\kappa_{H g g}, \kappa_{A g g}\right)=(1,0),(1,0.5)$, and $(1,-0.5)$

Figure 5.10: Comparison of $n_{\text {eff }} / n_{\text {tot }}$ in two dimensions $\left(\kappa_{H g g}\right.$ and $\left.\kappa_{A g g}\right)$ for morphing bases used to interpolate between values of $\kappa_{H g g}$ and $\kappa_{A g g}$. The SM equivalent scenario of $\kappa_{H g g}=1$ is marked with a dotted line. 
that parts of the coupling strength parameter space could be completely uncovered by such a configuration. Equivalent results were seen when building a basis from samples with $\left(\kappa_{H g g}, \kappa_{A g g}\right)=(1,0),(0,1)$, and $(1,1)$, with the main difference being that the patterns in $n_{\text {eff }} / n_{\text {tot }}$ were mirror images of those in Figures 5.9 and 5.10.

To attempt to produce more symmetrical distributions of $n_{\mathrm{eff}} / n_{\mathrm{tot}}$, the choice was made to fix $\kappa_{H g g}=1$ in the morphing basis and vary $\kappa_{A g g}$ alone, as in practice the EFT was implemented to constrain the degree of deviation from SM predictions. By using $\mathcal{C P}$-even/odd mixed samples with $\kappa_{A g g}= \pm 0.5$, it was found that $n_{\text {eff }} / n_{\text {tot }}$ was sufficiently high across the entire range of interest, with its distribution also being symmetrical for positive and negative $\kappa_{A g g}$, as shown in the right plot of Figure 5.9. Furthermore, when allowing $\kappa_{H g g}$ to vary from one, it was found that $n_{\text {eff }} / n_{\text {tot }}$ remained suitably large for the region enclosed by $0.5<\kappa_{H g g}<1.5$ and $-0.5<\kappa_{A g g}<0.5$, Outside of this enclosed region, the value of $\sigma_{\mathrm{ggF}}$ would deviate by at least $200 \%$ from SM predictions, therefore this range could easily be vetoed from consideration based on existing ATLAS cross section measurements.

Additional distributions of $n_{\text {eff }} / n_{\text {tot }}$ are shown in Figure 5.11 for two morphing bases which interpolate between values of $\kappa_{A V V}$ and $\kappa_{H V V}$ for $\mathrm{VBF}+\mathrm{VH}$ Higgs boson production, along with the chosen basis for $\kappa_{A g g}$ in ggF production. The VBF $+\mathrm{VH}$ morphing bases are each built from five samples containing $\kappa_{\mathrm{SM}}=1$ and $\kappa_{x V V}$ in $[-5,-2.5,0,2.5,5]$, while the ggF morphing basis is built from three samples with $\kappa_{\mathrm{SM}}=1$ and $\kappa_{A g g}$ in $[-0.5,0,0.5]$. For each morphing basis, a sufficiently good coverage in $n_{\text {eff }} / n_{\text {tot }}$ is observed across the entire domain spanned by the extrema of $\kappa_{\mathrm{BSM}}$. Outside of these regions, the value of $n_{\mathrm{eff}} / n_{\text {tot }}$ tends to drop rapidly, suggesting that the morphed representation of some observable cannot be considered reliable 


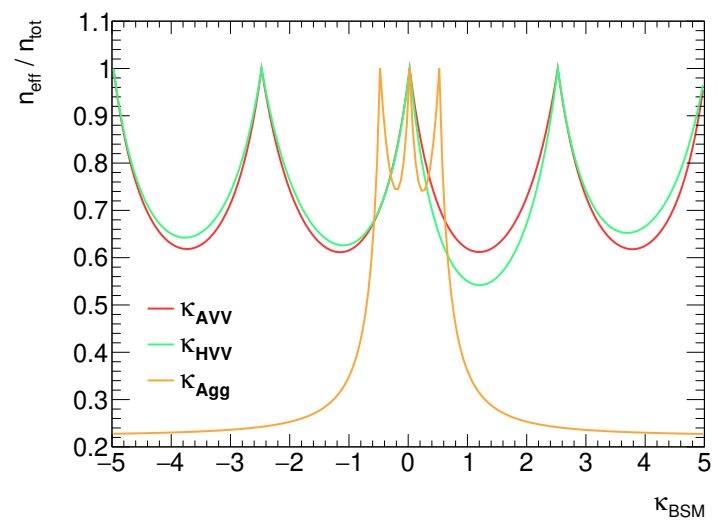

Figure 5.11: Distributions of $n_{\text {eff }} / n_{\text {tot }}$ for morphing bases built to interpolate beween values of $\kappa_{A V V}$ and $\kappa_{H V V}$ in $\mathrm{VBF}+\mathrm{VH}$ Higgs boson production, and $\kappa_{A g g}$ in ggF production.

outside the region covered by the morphing basis.

A further quantity of interest is the condition number of the morphing matrix, which effectively determines the sensitivity of the morphed output to initial conditions. A morphing matrix is considered to be "well-conditioned" if a small change in the morphing matrix (or base samples) results only in a small change in the morphed sample. It is evident that such a requirement is critical for the accurate representation of coupling configurations which deviate only weakly from the Standard Model. The condition number is computed for a square matrix $A$ of size $n^{2}$ as,

$$
\begin{gathered}
\operatorname{cond}(A)=\|A\|_{1} \cdot\left\|A^{-1}\right\|_{1} \text { where } \\
\|A\|_{1}=\max _{1 \leq j \leq n} \sum_{i=1}^{n}\left|a_{i j}\right| .
\end{gathered}
$$

Given these definitions, if the condition number is roughly $10^{k}$, then solving the equation $A \cdot \vec{x}=\vec{b}+\Delta \vec{b}$ for a small perturbation $\Delta \vec{b}$ yields a solution which loses $k$ significant figures relative to the solution of $A \cdot \vec{x}=\vec{b}$. For the one-dimensional mor- 
phing bases defined above to interpolate between different values of $\kappa_{A V V}$ and $\kappa_{H V V}$, for example, the condition number is 781 for the morphing matrices for each basis, while the condition number corresponding to the $\kappa_{A g g}$ morphing basis is 14 . Since most computational linear algebra is done using floating point or double precision, the loss of two or three points of precision should not have a meaningful effect on the measurements of these anomalous couplings, as they are made using observed data yields, which are at least $\mathcal{O}(1)$, by definition. 


\section{Chapter 6}

\section{Datasets, physics object}

\section{reconstruction, and event selection}

The processed output from the ATLAS detector is a collection of objects derived from measurements of different sub-detectors, such as calorimeter cells being grouped into clusters, or hits from the Inner Detector and Muon Spectrometer systems forming tracks, among other possibilities. The term physics object broadly refers to a grouping of one or more detector-specific objects, which provides a coherent description of the energy and trajectory of a particle (or particles). The process of building of these physics objects is known as reconstruction. This chapter describes the reconstruction methods for each physics object used in the $H \rightarrow Z Z^{*} \rightarrow 4 \ell$ analysis, as well as the approaches used to model $4 \ell$ signal and background processes. 


\subsection{Dataset and simulated signal samples}

At this point, it is worthwhile making the distinction between real data and simulated data, as these differences inform both the way that analyses function within ATLAS, and how predicted results are made for measurements. Real proton-proton collision data acquired through the ATLAS detector is reconstructed through the use of detector-level objects such as clusters and tracks. From these raw components, physics objects are formed, including electrons, muons, photons, and a variety of mesons. Using these individual particles, it is then possible to reconstruct composite objects, such as jets or lepton quadruplets. The data used for the studies presented in this work corresponds to $36.1 \mathrm{fb}^{-1}$ of integrated luminosity, recorded at $\sqrt{s}=13 \mathrm{TeV}$ in 2015 and 2016 during Run-II of the LHC.

Of course, prior to drawing conclusions from data, it is necessary to make predictions using "expected" results which inform both where and how ATLAS searches for the physics of interest. In most cases, the Monte Carlo method is used to produce simulations which allow for a detailed understanding of the physics of rare processes, and to optimize search criteria. Such simulations can also allow for variations of parameters such as particle masses, widths, and couplings, which are used extensively in BSM physics searches. The method of simulation used in ATLAS follows the same general principles as outlined in Chapter 5. Namely, the hard processes $p p \rightarrow X$ are simulated at parton-level using a variety of MC generators, which are then interfaced to a SMC program to include the UE and MPI aspects of the interaction, producing truth-level events. Lastly, the truth-level sample is propagated through a full simulation of the ATLAS detector based in GEANT4, along with simulated pile-up interactions, in order to emulate the luminosity conditions of a given LHC run period. 
Upon completion of the detector simulation stage, the simulated events are processed using the same ATLAS reconstruction software applied to data, creating reconstruction-level samples which are formatted identically to actual ATLAS data. In practice, the reconstruction-level events are typically reweighted using the bestknown theoretical cross section for a given process, and scaled by the integrated luminosity $\int \mathcal{L}$, such that the full sample reflects the number of events expected in data. Additional corrections for e.g. the instantaneous luminosity are often applied on an event-by-event basis, in order to adjust the simulation to better reflect the behaviour and detector conditions of the acquired data.

\subsubsection{SM Higgs boson signal modelling}

Samples of Standard Model ggF-mediated events are generated using PowHEG, via the NnLOPS process described in Section 5.1.1, with the PDFs provided by the PDF4LHC set [101]. Through the use of this technique, an accuracy of NNLO QCD is achieved in the inclusive cross section and process kinematics. However, in order to match the best-known prediction of the inclusive ggF cross section, the normalization of the full sample is scaled up to match the $\mathrm{N}^{3} \mathrm{LO}$ prediction of Ref. [31].

Samples of Standard Model VBF-mediated events are generated using PowhEG, via the VBFH process, which calculates the $H+2 j$ process at NLO QCD [102]. Similar to the ggF samples, the inclusive cross section of the VBF sample is reweighted to the best-known cross section prediction at NNLO QCD and NLO EW [31]. Samples of Standard Model $W / Z$ associated production events, including hadronic and leptonic final states for the weak boson decays [103], are produced using the PowHEG generator at NLO QCD via the MiNLO method [90], which merges 0-jet and 1-jet events 
to produce an overall higher order calculation. Each process is interfaced with the PYThia8 SMC generator using the AZNLO parameter set [104].

The Standard Model bbH and ttH production processes are modelled using MADGRAPH5_aMC@NLO [92], which generates diagrams for these processes at NLO QCD. Samples of ttH events are interfaced to the HERWIG++ SMC generator [105], while the bbH samples are interfaced to PYTHIA8. The cross sections of these samples are also scaled to their best-known predictions, with the ttH process being calculated to NLO QCD + NLO EW, and the bbH process calculated to NNLO or NLO, depending on the flavour of the outgoing quarks.

\subsubsection{Higgs boson signal with anomalous couplings}

To test for the presence of BSM physics effects in Higgs boson production and $4 \ell$ decay vertices, dedicated samples of $\mathrm{ggF}-$ and $\mathrm{VBF}+\mathrm{VH}-\mathrm{mediated} \mathrm{Higgs} \mathrm{signal}$ were produced using the Higgs Characterization model, as implemented in MADGraPH5_aMC@NLO. As described in Section 5.3, these samples introduce anomalous couplings in the $H g g$ and $H V V$ vertices which modify the $\mathcal{C P}$ state of the Higgs boson, affecting both the process cross sections, as well as the kinematics of the Higgs boson and any final state jets. In each modified sample, custodial symmetry is assumed, meaning that $\kappa_{x W W}=\kappa_{x Z Z}$, and for $\mathcal{C P}$-mixed states, the mixing an-

gle is taken to be $\alpha=1 / \sqrt{2}$, i.e. maximal $\mathcal{C P}$-mixing is assumed. Samples were produced at merged NLO QCD for ggF-mediated Higgs production using the FxFx prescription, while LO calculations were used for VBF+VH Higgs boson production. Interference between the $\mathrm{VBF}$ and hadronic $\mathrm{VH}$ production modes is accounted for within these samples, while leptonic VH production is treated separately. 


\begin{tabular}{ccccccc}
\hline \hline Description & $\cos (\alpha)$ & $\kappa_{\mathrm{SM}}$ & $\kappa_{\mathrm{Hvv}}$ & $\kappa_{\mathrm{Avv}}$ & \multicolumn{2}{c}{$\mathrm{MC}$ stat. } \\
\hline SM & 1 & 1 & 0 & 0 & $300 \mathrm{k}$ & $50 \mathrm{k}$ \\
Pure BSM & 1 & 0 & 10 & 0 & $100 \mathrm{k}$ & $50 \mathrm{k}$ \\
Pure BSM & 0 & 0 & 0 & 15 & $100 \mathrm{k}$ & $50 \mathrm{k}$ \\
Mixture & $\frac{1}{\sqrt{2}}$ & 1 & 0 & 5 & $100 \mathrm{k}$ & $50 \mathrm{k}$ \\
Mixture & $\frac{1}{\sqrt{2}}$ & 1 & 0 & 2.5 & $100 \mathrm{k}$ & $50 \mathrm{k}$ \\
Mixture & $\frac{1}{\sqrt{2}}$ & 1 & 0 & -5 & $100 \mathrm{k}$ & $50 \mathrm{k}$ \\
Mixture & $\frac{1}{\sqrt{2}}$ & 1 & 0 & -2.5 & $100 \mathrm{k}$ & $50 \mathrm{k}$ \\
Mixture & 1 & 1 & 5 & 0 & $100 \mathrm{k}$ & $50 \mathrm{k}$ \\
Mixture & 1 & 1 & 2.5 & 0 & $100 \mathrm{k}$ & $50 \mathrm{k}$ \\
Mixture & 1 & 1 & -5 & 0 & $100 \mathrm{k}$ & $50 \mathrm{k}$ \\
Mixture & 1 & 1 & -2.5 & 0 & $100 \mathrm{k}$ & $50 \mathrm{k}$ \\
Mixture & $\frac{1}{\sqrt{2}}$ & 1 & 2.5 & -5 & $100 \mathrm{k}$ & $50 \mathrm{k}$ \\
Mixture & $\frac{1}{\sqrt{2}}$ & 1 & 5 & 5 & $100 \mathrm{k}$ & $50 \mathrm{k}$ \\
Mixture & $\frac{1}{\sqrt{2}}$ & 1 & 5 & 6 & $100 \mathrm{k}$ & $50 \mathrm{k}$ \\
Mixture & $\frac{1}{\sqrt{2}}$ & 1 & -2.5 & -5 & $100 \mathrm{k}$ & $50 \mathrm{k}$ \\
Mixture & $\frac{1}{\sqrt{2}}$ & 1 & -5 & 5 & $100 \mathrm{k}$ & $50 \mathrm{k}$ \\
Mixture & $\frac{1}{\sqrt{2}}$ & 1 & -5 & -6 & $100 \mathrm{k}$ & $50 \mathrm{k}$ \\
\hline \hline
\end{tabular}

Table 6.1: List of input samples for the combined signal model of vector fusion production and associated production, used to build a morphing basis allowing the simultaneous introduction of $\kappa_{H V V}$ and $\kappa_{A V V}$ in the $H V V$ interaction vertex.

Due to the necessity of passing each signal sample through full pile-up and detector simulations, only a finite number of samples were generated with anomalous couplings, with the histogram morphing technique of Section 5.4 used to interpolate between hypotheses in both $\mathrm{ggF}$ and $\mathrm{VBF}+\mathrm{VH}$ production. The coupling configurations for the morphing bases used are given in Tables 6.1, 6.2, and 6.3 for $\mathrm{VBF}+\mathrm{VH}$ - and ggFmediated Higgs production. These particular configurations were derived through studies to optimize $n_{\text {eff }} / n_{\text {tot }}$ and $\operatorname{cond}(A)$, as described in Section 5.4. 


\begin{tabular}{lcccccc}
\hline \hline Description & $\cos (\alpha)$ & $\kappa_{\mathrm{Hgg}}$ & $\kappa_{\mathrm{SM}}$ & $\kappa_{\mathrm{Hzz}}$ & $\kappa_{\mathrm{Azz}}$ & MC stat. \\
\hline SM & 1 & 1 & 1 & 0 & 0 & $500 \mathrm{k}$ \\
Pure BSM & 1 & 1 & 0 & 1 & 0 & $100 \mathrm{k}$ \\
Pure BSM & $\frac{1}{\sqrt{2}}$ & 1 & 0 & 0 & 1 & $100 \mathrm{k}$ \\
Mixture & 1 & 1 & 1 & -6 & 0 & $100 \mathrm{k}$ \\
Mixture & $\frac{1}{\sqrt{2}}$ & 1 & 1 & 6 & 6 & $100 \mathrm{k}$ \\
Mixture & $\frac{1}{\sqrt{2}}$ & 1 & 1 & -6 & -6 & $100 \mathrm{k}$ \\
\hline \hline
\end{tabular}

Table 6.2: List of input samples for the gluon fusion signal model, used to build a morphing basis which allows the introduction of $\kappa_{H V V}$ and $\kappa_{A V V}$ in the $Z Z^{*}$ decay vertex.

\begin{tabular}{lccccc}
\hline \hline Description & $\cos (\alpha)$ & $\kappa_{\text {Hgg }}$ & $\kappa_{\text {Agg }}$ & $\kappa_{\mathrm{SM}}$ & MC stat. \\
\hline SM & 1 & 1 & 0 & 1 & $500 \mathrm{k}$ \\
Mixture & $\frac{1}{\sqrt{2}}$ & 1 & 0.5 & 1 & $100 \mathrm{k}$ \\
Mixture & $\frac{1}{\sqrt{2}}$ & 1 & -0.5 & 1 & $100 \mathrm{k}$ \\
\hline \hline
\end{tabular}

Table 6.3: List of input samples for the gluon fusion signal model describing $\mathcal{C P}$-mixing via the introduction of $\kappa_{A g g}$ in the ggF production vertex.

\subsection{Object reconstruction and selection}

Although nominally the $H \rightarrow 4 \ell$ decay channel is characterized by the selection of two pairs of opposite-sign, same flavour leptons, tracking and calorimetric information from other reconstructed physics objects (photons, jets) is used to both enhance sensitivity to different Higgs boson production modes, and enhance the mass resolution of the $4 \ell$ system where possible.

\subsubsection{Electron and muon reconstruction}

The reconstruction of electrons proceeds as described in Section 4.1 with the matching of tracks from the Inner Detector to clusters from the EM calorimeter. For the analysis results presented, the sliding window algorithm is used for EM cluster building and 
energy calibration. Muons in the region $0.1<|\eta|<2.5$ are reconstructed using tracks in the ID and MS, as described in Ref. [106]. In the region $|\eta|<0.1$, the MS has reduced coverage, so muons can only be reconstructed from ID tracks, and can be identified using either calorimeter deposits, or hits in the MS [107]. For $2.5<|\eta|<$ 2.7, only the MS can be used to identify and build muons. In the MS, individual track segments are combined into full tracks using an iterative, $\chi^{2}$ discriminant-based procedure, wherein track segments are grouped together and refitted combinatorially, until a group of segments is found which minimizes the $\chi^{2}$ discriminant. Conversely, in the ID, muon tracks are reconstructed in a manner identical to electron tracks, with the exception of not undergoing a GSF-like refitting procedure.

Since muons may leave tracks in the ID, MS, or both, multiple forms of muon reconstruction are used in ATLAS analyses, depending on the detector region in $|\eta|$. The various types of ATLAS muon reconstruction methods are summarized as follows, ordered roughly according to performance in terms of efficiency and resolution:

- Combined (CB) muons are formed from a combined fit of ID and MS tracks [107]

- Segment-tagged (ST) muons are formed from a combination of ID tracks with MS tracks existing only in specific MDT or CSC chambers in the MS [107]

- Silicon-associated forward (SiAF) muons combine MS tracks with ID "tracklets" from muons, the latter of which are truncated tracks, formed by muons which have passed only through the edges of the ID pixel detector system [108]

- Calorimeter-tagged (CT) muons consist of an ID track, along with an energy deposit in the calorimeter system resembling a minimum ionizing particle, such as a muon [107] 
In the region $0.1<|\eta|<2.5$, if available, CB muons are preferred for their high resolution and purity. Only $\mathrm{CT}$ and $\mathrm{ST}$ muons are available in $|\eta|<0.1$, since MS coverage is limited in this region. Similarly, only SiAF and ME muons are available in the region $2.5<|\eta|<2.7$ where ID coverage ends. If reconstructed, SiAF muons are preferred over other methods.

\subsubsection{Photon and jet reconstruction and selection}

Photons are reconstructed as described in Section 4.1, with unconverted photons built solely from sliding window clusters reconstructed in the EM calorimeter, and converted photons additionally using tracks in the Inner Detector. In this analysis, photons are not used to reconstruct any kind of final state decay, unlike the $H \rightarrow \gamma \gamma$ channel, for example. Rather, reconstructed photons are used primarily to recover the energy radiated by electrons and muons through material interactions with the Inner Detector, described in more detail in Section 6.3.

Jets are reconstructed from topological clusters of calorimeter cells using the anti- $k_{t}$ algorithm $[109,110]$, with nearby clusters grouped together inside cones of radius $R=0.4$ according to the inverse of their transverse momentum. The jets are then corrected for detector response and pile-up contamination, as described in Refs. [111, 112]. Tracks may also be grouped into jets using a procedure known as "ghost association" [113], which treats each track as a four-vector of infinitesimal magnitude during the jet reconstruction. Tracks are thereby assigned to the jet with which they are clustered [111].

To be used in the $4 \ell$ analysis, jets are required to have a minimum transverse momentum $p_{\mathrm{T}}>30 \mathrm{GeV}$, and lie within the pseudorapidity region $|\eta|<4.5$. Jets 
passing these acceptance cuts are further required to pass selection cuts designed to minimize the presence of pile-up noise. In particular, each jet is required to pass bad loose quality cuts [114], while also passing a cut on a discriminant calculated by the Jet Vertex Tagger (JVT) tool [115]. The JVT discriminant is based on a multivariate combination of two variables: the number of reconstructed vertices in the event; and the Jet Vertex Fraction (JVF), which is the fraction of the total momentum of the jet tracks associated to the reconstructed HS vertex ${ }^{1}$. The reconstruction of jets from $b$-quarks requires the use of dedicated algorithms to account for the greater degree of hadronization, described in Ref. [116].

Jet-electron overlap removal is also employed to avoid mistakenly reconstructing electrons as jets. In particular, jets are removed if $\Delta R(j, e)=\sqrt{\Delta \phi(j, e)^{2}+\Delta \eta(j, e)^{2}}<$ 0.2. This overlap removal is also applied to jets close to muons - in particular, a jet is removed either if $\Delta R(j, \mu)<0.1$, or if the jet has less than three tracks, and the energy and momentum differences between the muon and the jet are small $\left(p_{\mathrm{T}}^{\mu}>0.5 p_{\mathrm{T}}^{j}\right.$ and $\left.p_{\mathrm{T}}^{\mu}>0.7 p_{\mathrm{T}}^{j, \text { tracks }}\right)$.

\subsection{Event selection}

Among the millions of collisions produced by the LHC each second, only a chance few will result in $H \rightarrow Z Z^{*} \rightarrow 4 \ell$ decays. Event selection is used to isolate the signal of interest from the myriad of other signal and background processes recorded by ATLAS. Specific selection cuts have been tuned to isolate $4 \ell$ signal with a high efficiency and minimum of kinematic bias, as described in the following sections.

\footnotetext{
${ }^{1} \mathrm{~A}$ JVF value of 1 implies that $100 \%$ of the jet track momentum is associated to the HS vertex.
} 


\subsubsection{Pre-selection}

The pre-selection stage is meant to filter out events which do not meet basic usability criteria for an ATLAS physics analysis. For example, if a detector component is malfunctioning during a period of data taking, events recorded within this period will be vetoed, as there would be "holes" in the coverage of the detector, creating an artificial absence of measured energy and momentum. Periods of data taking are further organized into runs, which are composed of lumi-blocks, or one minute-long blocks of data acquisition. The Data Quality group in ATLAS keeps note of runs with detector malfunctions, abnormalities, etc. and produces a list of acceptable runs, the Good Runs List (GRL). Typically, any event under consideration must first be part of the GRL to propagate to further event selection in any physics analysis.

\subsubsection{Event-level selection}

The event-level selection is designed to filter out events which do not meet the basic criteria constituting a $4 \ell$ event candidate. The first requirement is made on the primary vertex $(\mathrm{PV})$, which is defined as the reconstructed vertex with the highest $\sum p_{\mathrm{T}}^{2}$ in a reconstructed event, which is generally the most likely result of a direct $p p$ collision. In particular, vertices are reconstructed using a $\chi^{2}$-based fit, wherein ID tracks with $p_{\mathrm{T}}>400 \mathrm{MeV}$ are used as input. All events under consideration must have a PV with at least two associated tracks. Events are furthermore required to pass at least one of the trigger requirements listed in Table 6.4. Worth noting is that the triggers presented were used in the final period of data taking in 2016, which may differ slightly from earlier implementations.

Preliminary event-level cuts are also made on reconstructed electrons and muons 


\begin{tabular}{|c|c|}
\hline Trigger name & Description \\
\hline & Single electron \\
\hline $\begin{array}{l}\text { e24_lhmedium_L1EM20VH } \\
\text { e60_lhmedium } \\
\text { e120_lhloose }\end{array}$ & $\begin{array}{l}\text { Single electron with } E \geq 24 \mathrm{GeV} \text { of medium } \\
\text { quality. The string " } \mathrm{L} 1^{*} \text { " represents level-1 } \\
\text { trigger requirements ( } \mathrm{V}=\text { varying thresholds } \\
\text { with } \eta, \mathrm{H}=\text { hadronic core isolation). }\end{array}$ \\
\hline & Di-electron \\
\hline 2e12_lhloose_L12EM10VH & Two electrons, as above. \\
\hline & Tri-electron \\
\hline e17_lhloose_2e9_lhloose & Three electrons, as above. \\
\hline & Single muon \\
\hline $\begin{array}{l}\text { mu20_iloose_L1MU15 } \\
\text { mu40 } \\
\text { mu60_0eta105_msonly }\end{array}$ & $\begin{array}{l}\text { One muon with } p_{\mathrm{T}}>20 \mathrm{GeV} \text { of loose } \\
\text { quality, and requiring the L1 } 15 \mathrm{GeV} \text { muon } \\
\text { trigger, or imposing }|\eta|<0.105 \text {. }\end{array}$ \\
\hline & Di-muon \\
\hline $\begin{array}{l}2 \mathrm{mu} 10 \\
\mathrm{mu} 18 \text { mu8noL1 }\end{array}$ & Two muons, as above. \\
\hline & Tri-muon \\
\hline $\begin{array}{l}3 \text { mu6 } \\
\text { 3mu6_msonly } \\
\text { mu18_2mu4noL1 }\end{array}$ & $\begin{array}{l}\text { Three muons, as above. The term msonly } \\
\text { indicates only a MS track is required. }\end{array}$ \\
\hline & Electron-muon \\
\hline $\begin{array}{l}\text { e17_lhloose_mu14 } \\
\text { 2e12_lhloose_mu10 } \\
\text { e12_lhloose_2mu10 } \\
\text { e24_medium_L1EM20VHI_mu8noL1 } \\
\text { e7_medium_mu24 }\end{array}$ & $\begin{array}{l}\text { Combined electron, muon trigger, as above. } \\
\text { In the } \mathrm{L} 1 \text { trigger label, I = electromagnetic } \\
\text { isolation requirements. }\end{array}$ \\
\hline
\end{tabular}

Table 6.4: Trigger list for the $H \rightarrow 4 \ell$ event selection, where rows have been grouped according to trigger type. 
to ensure a sufficiently high initial purity of $4 \ell$ candidates. Only electrons with $p_{\mathrm{T}}>7 \mathrm{GeV}$ and $|\eta|<2.47$ (i.e. within ID coverage) are used in this channel. Electron tracks are required to have at least one hit in the IBL, as well as a minimum number of track hits in the Pixel and SCT detectors. The reconstructed electrons are further required to satisfy loose identification criteria based on tracking and calorimeter information, as described in Section 4.1.3. The purpose of this identification scheme is to distinguish true signal electrons emerging from the PV from "fake" electrons that are the result of e.g. pile-up interactions. In particular, a cut is placed on the likelihood discriminant to achieve 95\% selection efficiency for true electrons.

The transverse momenta of $\mathrm{CB}, \mathrm{ST}, \mathrm{SiAF}$, and ME muons must satisfy $p_{\mathrm{T}}>$ $5 \mathrm{GeV}$, while CT muons are used if they have transverse momentum $p_{\mathrm{T}}>15 \mathrm{GeV} . \mathrm{A}$ priority when reconstructing muons from Higgs decays is the rejection of "fake" muons that are frequently the result of $\pi$ and $K$ meson decays. To improve the rejection of fake muons, a so-called loose working point is implemented, built by placing cuts on the number of ID and MS hits of a reconstructed muon. When both ID and MS tracks are available for a given muon, the working point is heavily defined by the $q / p$ significance, which is used to measure compatibility between the two kinds of tracks, and is calculated using the ID and MS track momenta and resolutions. A full description of the loose working point is found in Ref. [106].

Additionally, cuts are in place to reject muons which did not originate from the PV. In particular, these cuts are made on the impact parameters $d_{0}$, which is the distance between the track and the $z$-axis at the track perigee (point of closest approach to the beam line), and $z_{0}$, the $z$-coordinate of the perigee with respect to the PV. Figure 6.1 demonstrates the definition of $d_{0}$ and $z_{0}$ in the context of a particle track 


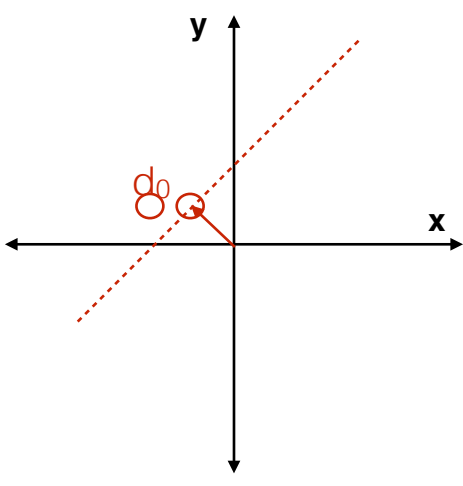

(a) $d_{0}$

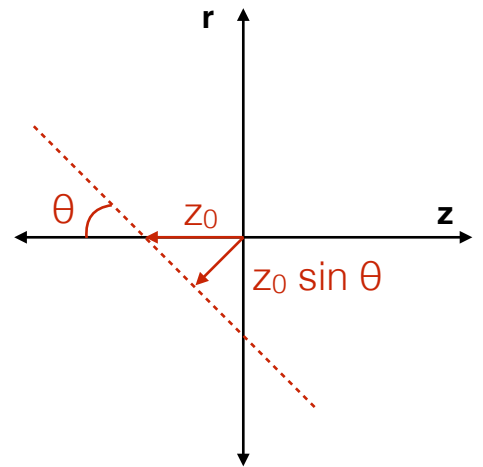

(b) $z_{0}$ and $z_{0} \sin \theta$

Figure 6.1: Definitions of the impact parameters $d_{0}, z_{0}$, and $z_{0} \sin \theta$ for a particle track crossing the $z$-axis / beam line.

crossing the $z$-axis / beam line. Muon tracks are required to have $\left|d_{0}\right|<1 \mathrm{~mm}$ and $\left|z_{0} \sin \theta\right|<0.5 \mathrm{~mm}$, with the exception of muons built only from MS tracks, for which no ID information is available. In practice, overlap is also possible between tracks from electrons and muons, necessitating the priority of one object over another. The overlap removal for $e, \mu$ works in two ways: The electron takes priority if it shares an ID track with a CT muon; otherwise, if an electron track overlaps any other kind of muon, only the muon is kept.

\subsection{Four lepton candidate selection}

After isolating the set of events passing the data quality pre-selection and object selection cuts, additional selection cuts are applied to both isolate events with topologies relevant to the $4 \ell$ analysis, and minimize experimental backgrounds. Namely, the remaining selection is focused on forming a Higgs boson candidate - or lepton quadruplet - composed of two same-flavour, opposite-sign (SFOS) lepton pairs. To 


\begin{tabular}{lc}
\hline \hline & Leptons and jets \\
\hline Muons & $p_{\mathrm{T}}>5 \mathrm{GeV},|\eta|<2.7$ \\
Electrons & $p_{\mathrm{T}}>7 \mathrm{GeV},|\eta|<2.47$ \\
Jets & $p_{\mathrm{T}}>30 \mathrm{GeV},|y|<4.4$ \\
Jet-lepton overlap removal & $\Delta R($ jet,$\ell)>0.1(0.2)$ for muons (electrons) \\
\hline \multicolumn{2}{c}{ Lepton selection and pairing } \\
\hline Lepton kinematics & $p_{\mathrm{T}}>20,15,10 \mathrm{GeV}$ \\
Leading pair $\left(m_{12}\right)$ & SFOS lepton pair with smallest $\left|m_{Z}-m_{\ell \ell}\right|$ \\
Subleading pair $\left(m_{34}\right)$ & remaining SFOS lepton pair with smallest $\left|m_{Z}-m_{\ell \ell}\right|$ \\
\hline \multicolumn{2}{c}{ Evelection $($ at most one quadruplet per channel) } \\
\hline Mass requirements & $50<m_{12}<106 \mathrm{GeV}$ and $12<m_{34}<115 \mathrm{GeV}$ \\
Lepton separation & $\Delta R\left(\ell_{i}, \ell_{j}\right)>0.1(0.2)$ for same- $($ different- $)$ flavour leptons \\
$J / \psi$ veto & $m\left(\ell_{i}, \ell_{j}\right)>5 \mathrm{GeV}$ for all SFOS lepton pairs \\
Mass window & $118 \mathrm{GeV}<m_{4 \ell}<129 \mathrm{GeV}$ \\
\hline \hline
\end{tabular}

Table 6.5: Event selection requirements defining the fiducial phase space of the $4 \ell$ analysis [52].

maximize the candidate reconstruction efficiency, the analysis selection proceeds in parallel for the four final states $(4 \mu, 2 e 2 \mu, 2 \mu 2 e, 4 e)$, meaning that several possible quadruplets can be formed. For each final state, a quadruplet is chosen if the three leading muons (electrons) pass $p_{\mathrm{T}}\left(E_{\mathrm{T}}\right)>20,15,10 \mathrm{GeV}$. The SFOS pair with an invariant mass closest to the $Z$ boson mass $\left(m_{12}\right)$ is denoted the leading pair, while the SFOS pair with an invariant mass second closest to the $Z$ boson mass $\left(m_{34}\right)$ is denoted the subleading pair. This approach leads to good reconstruction efficiency and purity; a mispairing of leptons within a quadruplet occurs for only about $1 \%$ of selected events with $4 \mu$ or $4 e$ final states [52].

After forming the candidate quadruplets, cuts on the lepton separation are imposed for each lepton, along with impact parameter requirements. The details of each selection cut are summarized in Table 6.5. Loose calorimeter- and track-based isolation cuts are also imposed on each lepton, which ensure that each quadruplet 
contains four distinct measured leptons. In particular, for the track-based isolation, the summed $p_{\mathrm{T}}$ of the tracks lying within a cone of $\Delta R \leq \min \left[0.3,10 \mathrm{GeV} / p_{\mathrm{T}}\right]$ $\left(\min \left[0.2,10 \mathrm{GeV} / E_{\mathrm{T}}\right]\right)$ around a given muon (electron) is required to be smaller than $15 \%$ of the lepton $p_{\mathrm{T}}\left(E_{\mathrm{T}}\right)$. Similarly, the summed $E_{\mathrm{T}}$ of the calorimeter deposits within a cone of $\Delta R \leq 0.2$ around the muon (electron) is required to be smaller than $30 \%(20 \%)$ of the lepton $p_{\mathrm{T}}\left(E_{\mathrm{T}}\right)$. Furthermore, the four leptons should originate from the same interaction vertex, so the reconstructed primary vertex and lepton tracks are subject to a $\chi^{2}$ requirement. If this requirement is met, then there is a high probability that all leptons in the quadruplet originated from the same interaction.

To further reject pile-up interactions, a cut is also applied to reject vertices displaced too far from the nominal interaction point by requiring $d_{0} / \sigma_{d_{0}}<3$ for muons and $d_{0} / \sigma_{d_{0}}<5$ electrons. In this case, $\sigma_{d_{0}}$ is the resolution on the impact parameter $d_{0}$ from the track reconstruction. Lastly, a veto is imposed on events where one dilepton pair within the quadruplet has an invariant mass in the range of the $J / \psi$ mass. If more than one quadruplet passes all selection requirements, the decay channel with the highest expected signal rate is selected, in the order: $4 \mu, 2 e 2 \mu, 2 \mu 2 e$ and $4 e$.

In general, it is possible for both muons and electrons to radiate photons during the $4 \ell$ decay process, as generalized in Figure 3.2. This behaviour is known as final state radiation (FSR), and if it is not recovered, it can negatively affect the resolution of the $4 \ell$ candidate invariant mass, $m_{4 \ell}$. Therefore, a search algorithm is in place to attempt to recover FSR by identifying candidate photons that can be associated to leptons in the quadruplet, which can be classified as "collinear" or "far", depending on their proximity to the quadruplet leptons.

Collinear photons are recovered only for muons emerging from the leading $Z$ 
boson candidate, if they lie within $\Delta R<0.15$ of the muon. A given collinear photon is accepted only if the leading dilepton invariant mass $m_{12}$ initially lies within $66<$ $m_{12}<89 \mathrm{GeV}$, such that post-FSR, it lies within $66<m_{12}<100 \mathrm{GeV}$. If multiple FSR photon candidates are found, only the highest $E_{\mathrm{T}}$ photon is used. If no collinear photons are found, an additional "far FSR" recovery routine is used, which searches in the region $\Delta R>0.15$ for electrons and muons in both the leading and subleading lepton pairs. Here, a photon can be recovered only for a quadruplet mass $m_{4 \ell}<$ $190 \mathrm{GeV}$, and only within the region $81<m_{\ell \ell}<100 \mathrm{GeV}$. In cases where multiple far FSR candidate photons are identified, only the highest $E_{\mathrm{T}}$ photon is used. Lastly, if far FSR photon candidates are identified for both $m_{12}$ and $m_{34}$, only the photon which alters the dilepton mass by smallest amount is taken.

The $H \rightarrow Z Z^{*} \rightarrow 4 \ell$ analysis also makes use of a $Z$ mass constraint, which uses the well-known $Z$ boson invariant mass $m_{Z}$, along with the natural width $\Gamma_{Z}$ and mass line shape to "correct" for degradation of the experimental $4 \ell$ mass resolution due to detector effects. In using such a correction, the kinematics of the $4 \ell$ system should be brought closer to what might be expected from the true underlying behaviour. The $Z$ mass constraint uses a maximum likelihood estimate (MLE) of the true $Z$ boson mass, denoted $m_{4 \ell}^{\text {cons }}$, derived in terms of the event-by-event $Z$ boson mass, along with the reconstructed $4 \ell$ candidate mass, and its corresponding resolution. This MLE is considered the constrained $Z$ boson mass, which is used to recompute the energies and momenta of the constituent leptons in the rest frame of the $Z$ boson according to the relation,

$$
\left(m_{Z}^{\text {cons }}\right)^{2}=\left(p_{1}^{\text {cons }}+p_{2}^{\text {cons }}\right)^{2}
$$


Furthermore, knowing the constrained $Z$ mass and lepton four momenta, a constrained four lepton mass, $m_{4 \ell}^{\text {cons }}$, can be derived in the Higgs boson rest frame using the remaining leptons as,

$$
\left(m_{4 \ell}^{\text {cons }}\right)^{2}=\left(p_{1}^{\text {cons }}+p_{2}^{\text {cons }}+p_{3}+p_{4}\right)^{2} .
$$

The constrained $4 \ell$ mass is taken to be the default $4 \ell$ candidate mass, such that the $4 \ell$ mass window is defined in terms of $m_{4 \ell}^{\text {cons }}$. Likewise, any analysis categories or discriminants that incorporate the leading $Z$ boson kinematics make use of $p_{1}^{\text {cons }}$ and $p_{2}^{\text {cons }}$, rather than their unconstrained variants.

\subsection{Analysis categories}

At the end of the $4 \ell$ candidate selection process, all events passing the selection form an inclusive sample. However, it is generally beneficial to divide this inclusive sample into exclusive sub-categories. For example, ggF-mediated Higgs boson production tends towards low jet multiplicities, while VBF and hadronic VH production tends to result in higher jet multiplicities. These subdivisions may enhance experimental sensitivity to various Higgs production modes, or even isolate phase space regions which are sensitive to BSM physics.

Starting from the inclusive sample, events are first divided into a ttH-enriched region if they contain $\geq 1$ jets which look sufficiently like reconstructed $b$-quarks. In addition to this requirement, the event must have $\geq 4$ jets, or one additional lepton ( $e$ or $\mu$ ) together with $\geq 2$ jets. The remaining events failing this selection are divided according to the number of final state leptons, with events containing $>4$ leptons 
divided into the leptonic VH-enriched category.

The remaining events are divided according to jet multiplicity into 0,1 , and $\geq 2$-jet categories. The 0-jet category enhances measurements of ggF-mediated Higgs boson production, as roughly $65 \%$ of gluon fusion events will have no final state jets at parton-level. All events with $\geq 2$ jets are required to have a minimum dijet invariant mass of $m_{j j}>40 \mathrm{GeV}$. The $\geq 2$-jet bin is subdivided into VBF and hadronic VHenriched regions by applying a cut on the dijet invariant mass at $m_{j j}=120 \mathrm{GeV}$, with VBF-enriched events falling into the region above $120 \mathrm{GeV}$. The 1-jet category also helps retain some VBF signal, as $\sim 20 \%$ of reconstructed VBF events have a jet which is either not reconstructed, or falls outside the fiducial region.

Lastly, within the exclusive 1 and $\geq 2$-jet categories, events are further subdivided into regions of $p_{\mathrm{T}}^{4 \ell}$ or $p_{\mathrm{T}}^{j_{1}}$, the use of which can help distinguish the different SM Higgs boson production modes. In particular, the 1-jet category is divided into three bins: $p_{\mathrm{T}}^{4 \ell}<60 \mathrm{GeV} ; 60<p_{\mathrm{T}}^{4 \ell}<120 \mathrm{GeV} ;$ and $p_{\mathrm{T}}^{4 \ell}>120 \mathrm{GeV}$. The low $p_{\mathrm{T}}$ categories tend to enhance sensitivity to ggF production, while the higher $p_{\mathrm{T}}$ bins isolate VBF events with only one reconstructed jet, as the Higgs boson tends to be produced around $|\eta| \sim 0$ in tree-level VBF production. Likewise, the $\geq 2$-jet, VBF-enriched bin is separated into two categories above and below $p_{\mathrm{T}}^{j_{1}}=200 \mathrm{GeV}$, with the high $p_{\mathrm{T}}$ bin being particularly sensitive to BSM physics effects in the $H V V$ interaction vertex.

A flowchart of the analysis categorization is shown in Figure 6.2. For the analysis targeting constraints on anomalous couplings using the Higgs Characterization model, the hadronic VH-enriched category is further subdivided into bins of $p_{\mathrm{T}}^{4 \ell}<150 \mathrm{GeV}$ and $p_{\mathrm{T}}^{4 \ell}>150 \mathrm{GeV}$, a change which enhances sensitivity to the presence of BSM couplings in $H V V$ interaction vertex. 


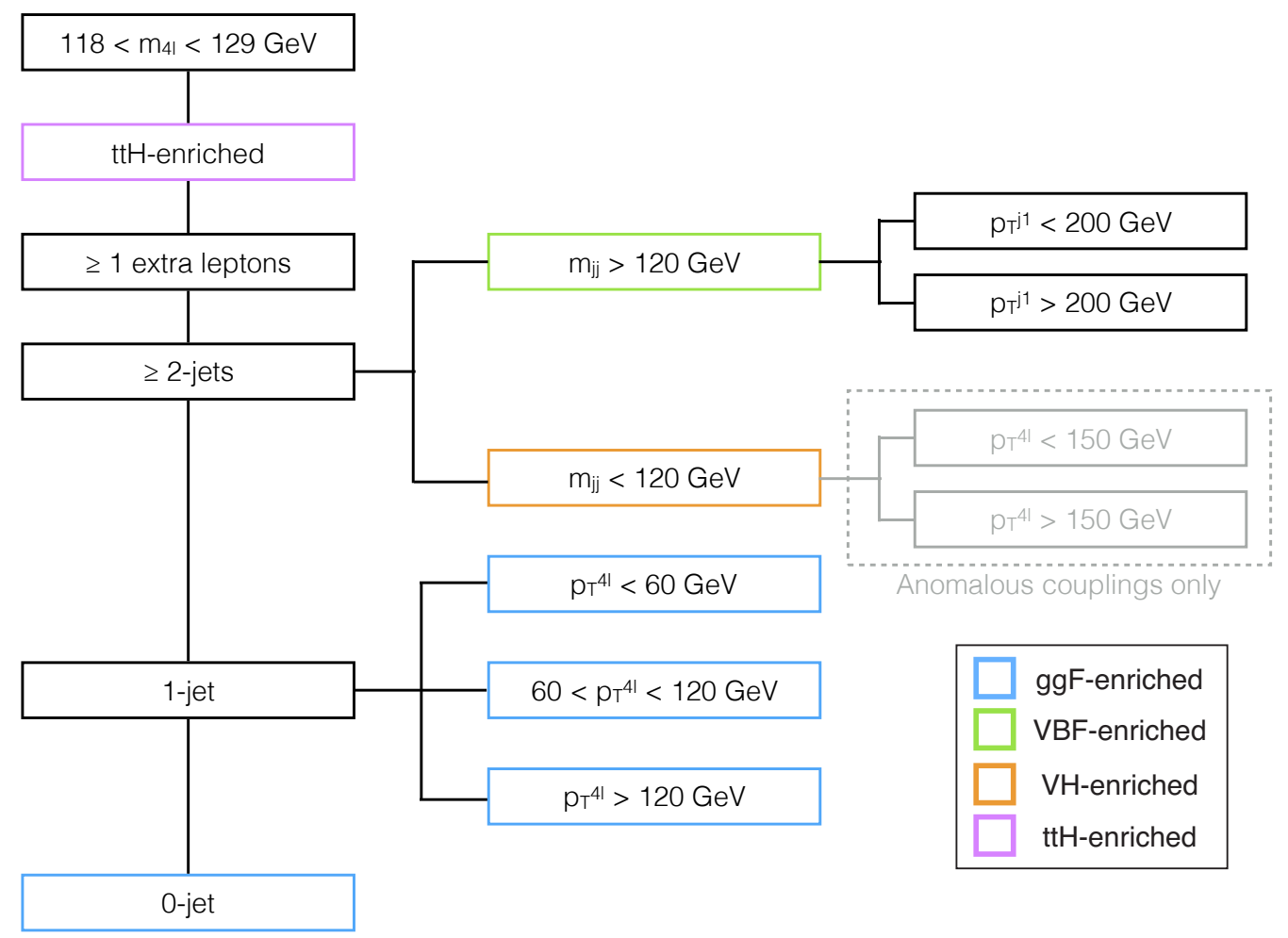

Figure 6.2: Flow chart of event categorization in the $4 \ell$ analysis for $\sqrt{s}=13 \mathrm{TeV}$. 


\subsection{Background estimation and modelling}

As previously established, the $H \rightarrow Z Z^{*} \rightarrow 4 \ell$ process tends to feature rather low background levels in the invariant mass region around $m_{4 \ell}=125 \mathrm{GeV}$. Although the background sources for this channel are relatively small, it is still important to model them with sufficient accuracy, in order to properly estimate the total event rates predicted by the Standard Model. The $Z Z^{*}$ continuum background is the largest source of irreducible, non-resonant background in the $H \rightarrow 4 \ell$ decay channel, which can emerge from quark-antiquark, or gluon-gluon interactions. These sources are considered irreducible as they mimic the multiplicity and topology of the final state leptons in the signal process.

In order to model the quark-antiquark annihilation-mediated process, simulated background samples were produced using the SHERPA 2.2 generator $[117,118,119]$, with the calculations done at NLO accuracy for 0- and 1-jet final states, and LO accuracy for 2- and 3-jet final states. Merging of the different jet multiplicities was also performed using SHERPA, which functions as an SMC generator [120] using the MePs@NLO prescription [121]. NLO electroweak corrections were also applied as a function of the invariant mass of the $Z Z^{*}$ system $[122,123]$. The gluon-induced $Z Z^{*}$ production process is modelled at LO in QCD using the GG2VV generator [124]. To account for missing higher-order QCD effects in the calculation of the $g g \rightarrow Z Z^{*}$ process, the cross section of the sample is scaled up by a factor of $1.7[125,126,127]$.

Along with the $q q Z Z / g g Z Z$ processes, minor background contributions exist from $t \bar{t} V$ and triboson production, which were also modelled using MC simulations, and normalized to the highest-order SM prediction available. In particular, the triboson backgrounds $Z Z Z, W Z Z$, and $W W Z$, each with four or more prompt leptons, 
were modelled using SHERPA 2.1.1, while the $t \bar{t} V$ background was modelled using Powheg interfaced to the Pythia 6 SMC generator.

\subsubsection{Reducible background estimation}

In contrast to the irreducible $Z Z^{*}$ background contribution, reducible backgrounds can have their effects mitigated by sufficiently strict cuts on the multiplicity and kinematics of the four final state leptons. The reducible background sources in the $Z Z^{*}$ decay channel include $Z+$ jets, $t \bar{t}$, and $W Z$ processes, whose contributions in the signal region (SR) are estimated using dedicated control regions (CRs) in data, which contain little to no signal by design. The estimates are produced separately for events with different flavours of the subleading lepton pair, i.e. $\ell \ell \mu \mu$ or $\ell \ell e e$, where $\ell \ell$ denotes the leading $\mu \mu$ or ee pair. To maximize the available background data, no selection is imposed on the $4 \ell$ invariant mass in the CRs. The backgrounds estimates are done in two stages: Firstly, estimates are made for the inclusive event selection, i.e. prior to event categorization; secondly, estimates are made with the background data divided into the reconstruction-level categories defined in Section 6.5.

The reducible $\ell \ell \mu \mu$ background is composed of $4 \ell$ objects with at least one jet containing a muon from secondary decays of $\pi / K$ mesons, or heavy-flavour hadrons. The $Z+$ jets background contribution encompasses a heavy quark flavour $(Z+\mathrm{HF})$ component, containing jets with $b$ - or $c$-quark content, and a light quark flavour $(Z+\mathrm{LF})$ component from $\pi$ or $K$ meson decays. These components of the $Z+$ jets background and the $t \bar{t}$ contribution are extracted using mutually exclusive CRs, which are formed by relaxing the $\chi^{2}$ requirement on the primary vertex fit mentioned in Section 6.3, and by inverting or relaxing the isolation and/or impact parameter requirements on 
the subleading muon pair. In these regions, a likelihood-based fit to $m_{12}$ is performed to obtain the background yield. Once the yields in the $t \bar{t}, Z+\mathrm{HF}$, and $Z+\mathrm{LF}$ CRs are established, a simulation-based method is used to extrapolate to the SR in a manner that depends on the impact parameter selection criteria, and efficiency of the isolation cuts. The contribution from $W Z$ production is estimated using simulation via POWHEg interfaced to PyThia8.

The reducible $\ell$ lee background contribution is classified into three processes: jets misidentified as an electron $(f)$; electrons from converted photons $(\gamma)$; and electrons from semileptonic decays of heavy quarks $(q)$. The contribution of the $q$ component is obtained from simulation, while $f$ and $\gamma$ components are obtained from CRs known as $3 \ell+X$ regions, in which three leptons pass the full analysis selection, while the lowest$E_{\mathrm{T}}$ electron (denoted by $X$ ) in the subleading electron pair passes a simplified electron identification cut, in which only the track hit requirement is applied. Generally speaking, if a fake electron is present in a quadruplet, it tends to be the lowest- $E_{\mathrm{T}}$ electron, hence their use in this CR.

To suppress contributions from the irreducible $Z Z^{*}$ process, only electrons with same-sign charge are considered for the subleading electron pair in this CR. To separate the $\gamma$ and $f$ background components, a template fit is used, which distinguishes the processes based on the number of track hits in the innermost Pixel layer for the associated track. The templates for the $\gamma$ and $f$ background contributions are obtained from simulated $Z+X$ events, composed of an on-shell $Z$ boson decay candidate together with an electron $X$, which are selected using the same criteria as in the $3 \ell+X \mathrm{CR}$. The simulated $Z+X$ events are also used to extrapolate the $f$ and $\gamma$ background contributions from the $\mathrm{CR}$ to the $\mathrm{SR}$, after correcting the simulation 


\begin{tabular}{lcc}
\hline \hline & \multicolumn{2}{c}{ Best prediction scale factors } \\
Category & ggF + bbH + ttH & VBF + VH \\
\hline 0-jet & 1.6 & 1.3 \\
1-jet, $p_{\mathrm{T}}^{H}<60 \mathrm{GeV}$ & 1.5 & 1.3 \\
1-jet, $p_{\mathrm{T}}^{H} \in[60,120] \mathrm{GeV}$ & 1.5 & 1.4 \\
1-jet, $p_{\mathrm{T}}^{H}>120 \mathrm{GeV}$ & 1.2 & 1.5 \\
2-jet VBF-enriched, $p_{\mathrm{T}}^{j_{1}}<200 \mathrm{GeV}$ & 1.3 & 1.0 \\
2-jet VBF-enriched, $p_{\mathrm{T}}^{j_{1}}>200 \mathrm{GeV}$ & 1.0 & 1.0 \\
2-jet VH-enriched, $p_{\mathrm{T}}^{H}<150 \mathrm{GeV}$ & 1.5 & 1.3 \\
2-jet VH-enriched, $p_{\mathrm{T}}^{H}>150 \mathrm{GeV}$ & 0.9 & 1.4 \\
VH-leptonic & 4.5 & 1.3 \\
ttH-like & 6.0 & 2.0 \\
\hline \hline
\end{tabular}

Table 6.6: List of per-category "best prediction" scale factors for Higgs signal samples with anomalous couplings.

to match the data, using dedicated samples of $Z+X$ events.

\subsection{Best prediction scaling for signal with anoma- lous couplings}

The Higgs signal samples used in the anomalous couplings studies are generated at a lower order than those used for the SM analysis. In order for the signal model to converge to the best-known SM signal rate predictions when all anomalous coupling strengths are zero, a so-called "best prediction" scale factor method is implemented for the different Higgs boson production modes. In essence, $k$-factors are computed on a per-category basis for each signal mode, which are assumed to be the same for SM and BSM scenarios. A given scale factor is defined as the ratio of expected number of events in a category obtained from the SM prediction, $S_{\text {POWHEG,SM, }}$ and from the SM coupling point sample, $S_{\mathrm{MG5,SM}}$, derived using MADGrAPH5_aMC@NLO. 
Accordingly, the scale factor for the $j^{\text {th }}$ category is computed as,

$$
f_{j}=\frac{\int S_{j}^{\mathrm{POWHEG}, \mathrm{SM}}}{\int S_{j}^{\mathrm{MG}, \mathrm{SM}}} .
$$

The corrected signal model $S_{j}^{\text {final }}(\vec{\kappa})$ is then defined as,

$$
S_{j}^{\mathrm{final}}(\vec{\kappa})=S_{j}^{\mathrm{POWHEG}, \mathrm{SM}}-f_{j} \cdot S_{j}^{\mathrm{MG} 5, \mathrm{SM}}+f_{j} \cdot S_{j}(\vec{\kappa})
$$

with the BSM signal model $S_{j}(\vec{\kappa})$ obtained using the morphing method described in Section 5.4. For events divided solely into reconstruction-level categories, the best prediction scaling of the signal model simplifies to beome,

$$
S_{j}^{\text {final }}(\vec{\kappa})=f_{j} \cdot S_{j}(\vec{\kappa})
$$

Separate scale factors are computed for ggF combined together with ttH and bbH production, and for $\mathrm{VBF}+\mathrm{VH}$ production, as shown in Table 6.6. 


\section{Chapter 7}

\section{The VBF $H \rightarrow Z Z^{*} \rightarrow 4 \ell$ multivariate analysis in ATLAS}

Vector boson fusion is among the rarer ways to produce a Higgs boson, with its production cross section being roughly twelve times smaller than that of gluon fusion. However, its highly characteristic final state (i.e. a central Higgs boson in association with two forward jets) means that the VBF production mode is an ideal candidate for the use of multivariate techniques, allowing for the enhancement and extraction of a small VBF "signal" on top of a large ggF "background". The use of multivariate techniques in VBF searches is not a new phenomenon in ATLAS; the $H \rightarrow \gamma \gamma$ analysis group, for example, used such techniques during Run-I to disentangle VBF production from the large, irreducible diphoton backgrounds present in the channel [128]. Likewise, the $H \rightarrow Z Z^{*} \rightarrow 4 \ell$ analysis made cursory use of a multivariate VBF discriminant in previous analyses during Run-I [129]. This chapter presents work done by the author to build an optimized multivariate discriminant which separates VBF- and ggF-mediated $H \rightarrow Z Z^{*} \rightarrow 4 \ell$ events for Run-II of the LHC, target- 
ing a centre-of-mass energy of $\sqrt{s}=13 \mathrm{TeV}$. This chapter also details the technical implementation of the resultant tools into the larger $Z Z^{*}$ analysis framework.

The work detailed in this chapter was contemporaneous with prospective studies to characterize VBF $H \rightarrow 4 \ell$ production at the High-Luminosity LHC, which extends the lifetime of the LHC past 2030 in order to record a total integrated luminosity of $3 \mathrm{ab}^{-1}$ at $\sqrt{s}=14 \mathrm{TeV}$. To acquire this large volume of data, the target luminosity levels at the HL-LHC will result in pile-up of $\langle\mu\rangle \sim 140$ to 200, affecting the visibility of both VBF and ggF Higgs production modes. Appendix D details the application of the $\mathrm{VBF} H \rightarrow 4 \ell$ multivariate analysis to simulations of these experimental conditions. However, the derivation of the multivariate analysis itself is presented here as a separate chapter, as the work is motivated mainly by the underlying physics of the processes under study, rather than specific luminosity or detector conditions.

\subsection{Boosted decision trees}

The multivariate classifier used in this analysis is derived using the boosted decision tree (BDT) method, as implemented in the TMVA package [82]. The BDT method uses multiple binary decision trees (as shown in Figure 7.1) to form a robust, statistically stable classifier which discriminates signal from background events in a data sample [82]. Each binary tree makes decisions using a set of user-provided discriminating variables, and is trained using distinct, non-overlapping signal and background samples. The function of each tree is to use a series of 'yes/no' decisions to classify individual events as signal-like or background-like.

The result from each tree (the leaf node) is combined via weighted average into a single discriminant, known as the BDT score [82]. This score varies nominally on 


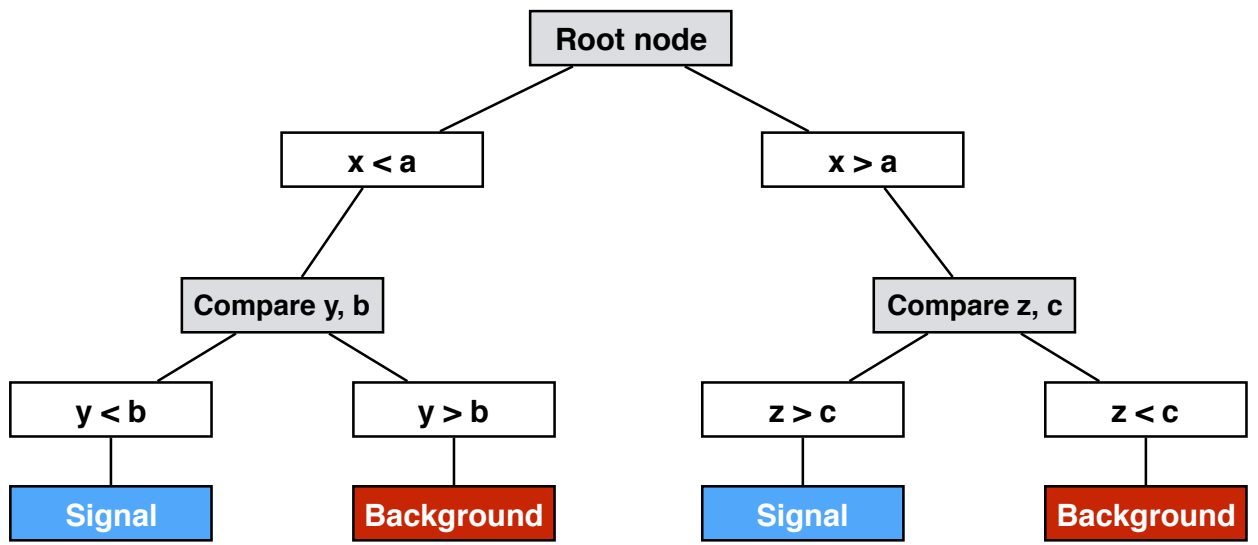

Figure 7.1: Schematic diagram of a binary decision tree.

$[-1,+1]$, with background-like events assigned highly negative scores, and signal-like events assigned highly positive scores. The 'boosting' aspect derives from the fact that the weight of each tree is proportionate to its rate of misclassification (i.e. poor signal/background discrimination yields a low weight, and vice versa).

The form of boosting used in this analysis is gradient boosting [130], which treats the individual trees as a sum of terms in a function expansion approach. Considering a set of input variables $\mathbf{x}=\left(x_{1}, x_{2}, \ldots, x_{n}\right)$, gradient boosting seeks to model the BDT score as an output variable $y$, mapped to $\mathbf{x}$ through a function $y_{i}=\hat{F}\left(\mathbf{x}_{i}\right)$. This boosting method was chosen for the multivariate analysis as it tends to isolate signal- and background-like events in opposite ends of the BDT score spectrum by default. Furthermore, gradient-boosted decision trees are also less susceptible to overtraining [82] - an inefficiency wherein the BDT learns only from a narrow region of phase space, or statistical fluctuations due to a low training sample size.

There are two interrelated tasks to consider when developing a BDT-based analysis. Firstly, signal and background training samples must be selected that both model well the underlying physics of the system under study, and are large enough to avoid 
overtraining effects in the BDT response. Secondly, training variables must be chosen to differentiate signal from background efficiently, and show no bias towards the quantity (or quantities) of interest in the analysis. The successful implementation of these two elements will produce an optimized, robust BDT-based analysis.

\subsection{Input sample selection}

In order to derive an effective BDT classifier, the input training samples must properly describe both the kinematics of the training variables, as well as the correlations between variables. Inadequate or incorrect modelling of the correlations between training variables can lead the BDT to make suboptimal decisions, meaning reduced signal efficiency or purity when the MVA is applied to real data. At the same time, Monte Carlo samples used for ATLAS analyses are required to undergo both detector and pile-up simulation, as described in Section 4.8, to properly account for the effects of both object reconstruction efficiency and resolution on signal and background event yields. These aspects of the simulation are particularly time-consuming and computationally intensive, meaning that reconstruction-level Monte Carlo samples tend to only be as large as is necessary to provide sensible yield predictions in each analysis category, resulting oftentimes in samples with $\mathcal{O}(100 \mathrm{k})$ events or less. Accordingly, for ggF events with two jets, which constitute approximately $12 \%$ of the total cross section [31], the training sample size could be as small as $\mathcal{O}(10 \mathrm{k})$ events.

Therefore, the decision was made to consider two cases when deriving the BDT to separate ggF and VBF Higgs boson production. Firstly, the derivation and optimization of the BDT would take place using truth-level samples, which would allow for the efficient production of large Monte Carlo training samples, of size $\mathcal{O}(1 \mathrm{M})$ events 
or more. The large sample size would allow for all regions of the phase space to be adequately probed by the BDT, and provide an accurate representation of the underlying correlations between kinematic variables. In a sense, this approach also allows for a more "physics-motivated" BDT training, as no detector effects are considered in either the shapes or correlations of training variables. Secondly, once an optimized multivariate approach was derived which reflects the true underlying physics of the ggF and $\mathrm{VBF}$ processes, the BDT would be re-trained using fully detectorsimulated, reconstruction-level samples of each process. Such an approach allows for reconstruction-level effects to be factored into the decisions made by the BDT, while also preventing the optimization process from being sensitive to detector efficiency or luminosity conditions.

To justify such an approach, the shapes and correlations of each training variable would require comparisons at truth- and reconstruction-level to ensure that the underlying physics of each process are properly represented by the reconstructionlevel Monte Carlo. To model VBF signal at both truth- and reconstruction-level, the Powheg VBFH generator was used, as described in Section 6.1.1. Conversely, to model truth-level dijet ggF production, the POwHEG HJJ generator was used, which produces $H+2 j$ events at NLO QCD, while at reconstruction-level, the NNLOPS method was used, which produces dijet events at LO. Although the kinematic differences between $H+2 j$ calculations at LO and NLO QCD are relatively small (as shown at truth-level in Figure 5.2), the physics-motivated nature of the BDT derivation meant that each training sample should be as theoretically accurate as possible. A comparison of the truth-level and reconstruction-level distributions of key dijet kinematic variables are shown in Figures 7.2 and 7.3, with good agreement seen be- 
tween truth and reconstruction-level shapes for both VBF and ggF production.

As a brief technical aside, a BDT is derived in two fundamental stages: a training stage where the classifier is derived based on input signal and background samples; and a testing stage where the trained classifier is applied to separate testing samples to evaluate the compatibility of the resultant signal/background discriminant distributions with those of the training samples. In order to avoid inducing biases in the training, the samples used for training and testing the BDT are required to be orthogonal, or have no events present in both training and testing samples. The separation of input samples into training and testing components was done using the default, random splitting algorithm in TMVA, ensuring that non-overlapping sub-samples were produced, which are populated by essentially random selections of events.

\subsection{Input variable selection}

The VBF process provides a rather unique kinematic signature at tree-level, consisting of two final state jets which tend to have high rapidity, along with a Higgs boson that tends to be central and low-momentum. Therefore, the kinematic variables which stand to best characterize this signal process are those which exploit its unique characteristics. For example, the dijet invariant mass $m_{j j}=\sqrt{\left(E_{j_{1}}+E_{j_{2}}\right)^{2}-\left(\vec{p}_{j_{1}}+\vec{p}_{j_{2}}\right)^{2}}$ tends to be quite large in VBF, owing to the fact that the two jets are highly separated in rapidity, to the point that the sum $\vec{p}_{j_{1}}+\vec{p}_{j_{2}}$ becomes nearly zero. Similarly, the transverse momentum of the combined Higgs boson - dijet system, $p_{\mathrm{T}}^{H j j}$, tends towards small values, owing to the small dijet system $p_{\mathrm{T}}$ from forward jets, and the low momentum of the Higgs boson itself.

Various numerical approaches can be taken to assess the discriminating power 

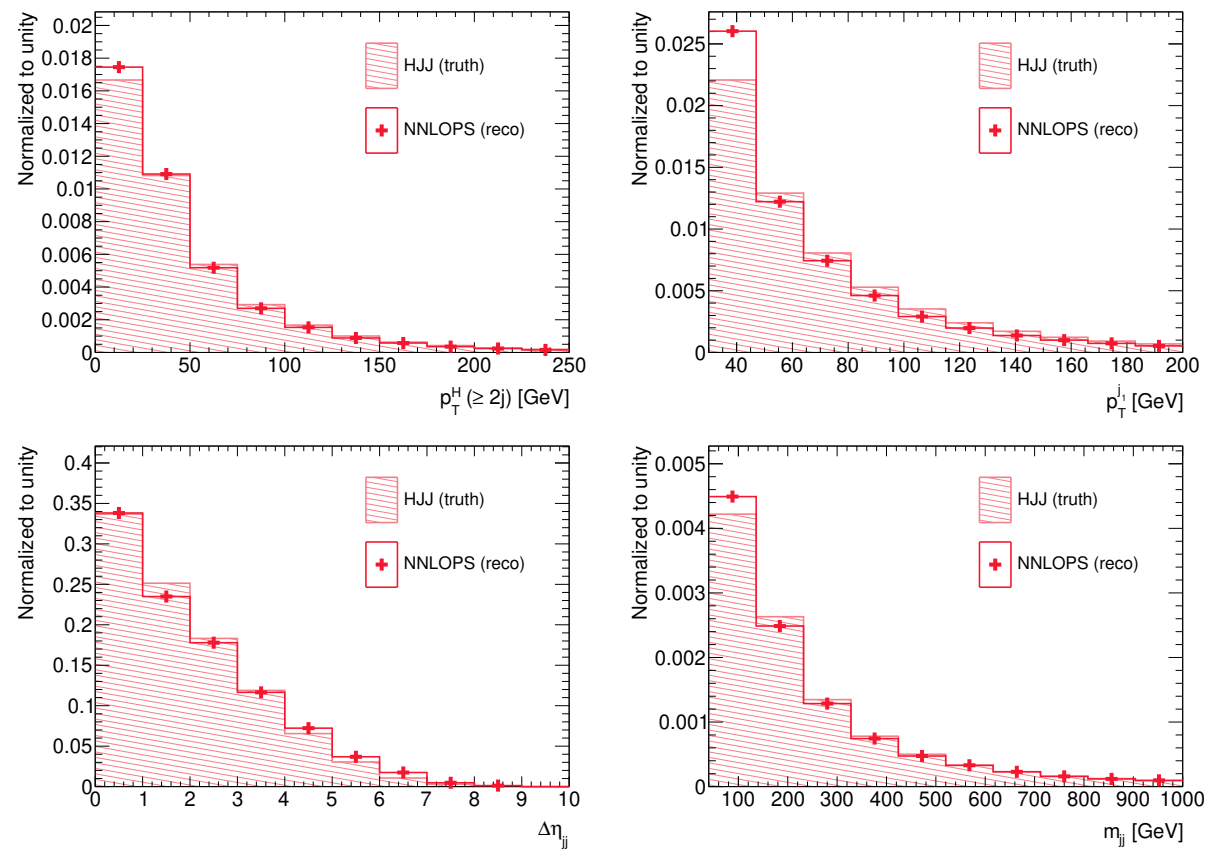

Figure 7.2: Comparison of truth- and reconstruction-level kinematics for ggF $H+2 j$ events. The truth-level events are generated at NLO QCD using the PowHEG HJJ generator, while the reconstruction-level events are generated at LO using the POWHEG NNLOPS generator.
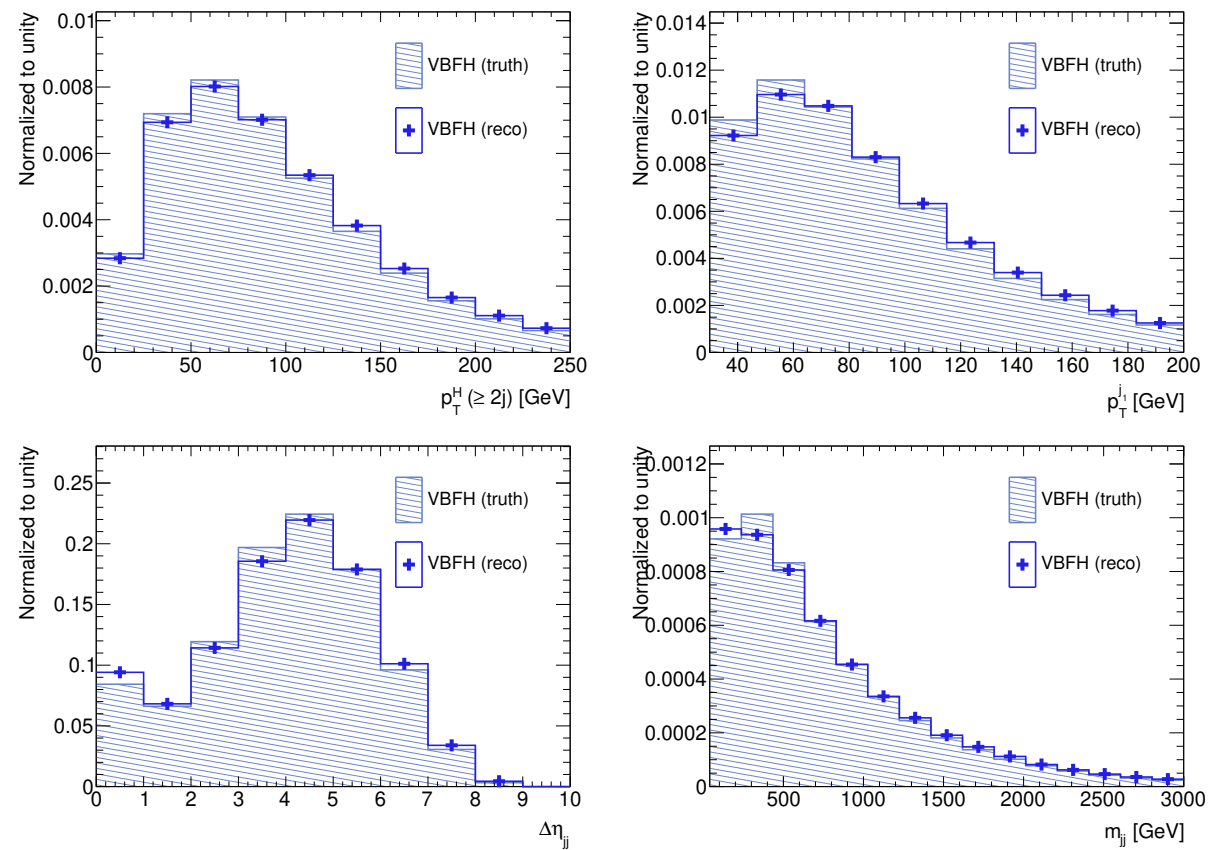

Figure 7.3: Comparison of truth- and reconstruction-level kinematics for VBF $H+2 j$ events. Both truth- and reconstruction-level events are generated using the POWHEG VBFH generator. 
of a given training variable. In TMVA, two particular quantities are used to assess this property. The shape separation $\left\langle S^{2}\right\rangle$ is used as an initial metric of performance, calculated as,

$$
\left\langle S^{2}\right\rangle=\frac{1}{2} \sum_{i=1}^{N_{\text {bins }}} \frac{\left(s_{i}-b_{i}\right)^{2}}{s_{i}+b_{i}} .
$$

where $s$ and $b$ represent binned probability density functions for the "signal" (VBF) and "background" (ggF) processes, respectively. Worth noting is that this variable is entirely shape-based, such that separation is zero for identical signal and background shapes, and one for shapes with no overlap. Therefore, differences in the overall normalization between the two samples are ignored, providing a highly intuitive, kinematically-motivated measure of the separability of the two processes.

The other quantity used to assess discriminating power is the importance, calculated internally within TMVA after the first pass of BDT training. The importance is a BDT-specific quantity, and quantifies how frequently a variable is used to make decisions. It is computed for a given variable as the frequency of its use in node splitting, weighted by both the number of events in each node, and the separation gain-squared it achieved [82]. So, the ideal set of training variables will demonstrate high individual shape separation, and a relatively equal balance of importance in their usage within the BDT.

To begin, a number of training variables were initially considered based around the Higgs, jet, and dijet kinematics, with studies performed to shrink an initially large list using $\left\langle S^{2}\right\rangle$ and the BDT importance as metrics. Shape distributions of the most performant variables of this initial list are shown in Figure 7.4. Aside from the more straightforward single jet and dijet-related variables, a number of variables also 
represent the composite Higgs-dijet system, such as $\eta_{Z Z}^{*}$, which is defined as,

$$
\eta_{Z Z}^{*}=\left|\frac{\eta_{j_{1}}+\eta_{j_{2}}}{2}-\eta_{Z Z}\right|
$$

and the minimum $\Delta R\left(j_{1}, j_{2}, Z_{1}, Z_{2}\right)$ (or $\Delta \mathrm{R}_{j Z}^{\min }$ ), which is defined as the minimum $\Delta R$ among the following choices,

$$
\Delta R\left(j_{1}, Z_{1}\right), \Delta R\left(j_{1}, Z_{2}\right), \Delta R\left(j_{2}, Z_{1}\right), \Delta R\left(j_{2}, Z_{2}\right) .
$$

To demonstrate the performance of each variable in a multivariate context, a BDT was trained using all chosen discriminating variables. The shape separation and importance for each variable is given in Table 7.1, with the variables ranked in order of decreasing shape separation. The most obvious conclusion from Table 7.1 is that $\Delta \eta_{\mathrm{jj}}$ is both the most separating variable, and used most frequently by the BDT. Such a result is not particularly surprising; considering that the tell-tale sign of VBF is two high energy, forward jets in conjunction with a central Higgs boson, it should be expected that this characteristic is exploited prominently by the BDT training.

However, it can also be gathered from this table that variables with high shape separation can in practice have low importance to the BDT. For instance, $p_{\mathrm{T}}^{H j j}$ is the third most separating training variable, but is only sixth in terms of importance, implying that it is either less frequently used by the BDT to make decisions, or that much of the decisions using this variable have a relatively small effect on the final signal purity. Likewise, $m_{\mathrm{jj}}$ is the fourth most important variable, a result which is likely due to its high correlation with $\Delta \eta_{\mathrm{j} j}$, conferring it a slight degree of redundancy.

Conversely, the second most important variable is found to be $\eta_{Z Z}^{*}$, which is ranked 

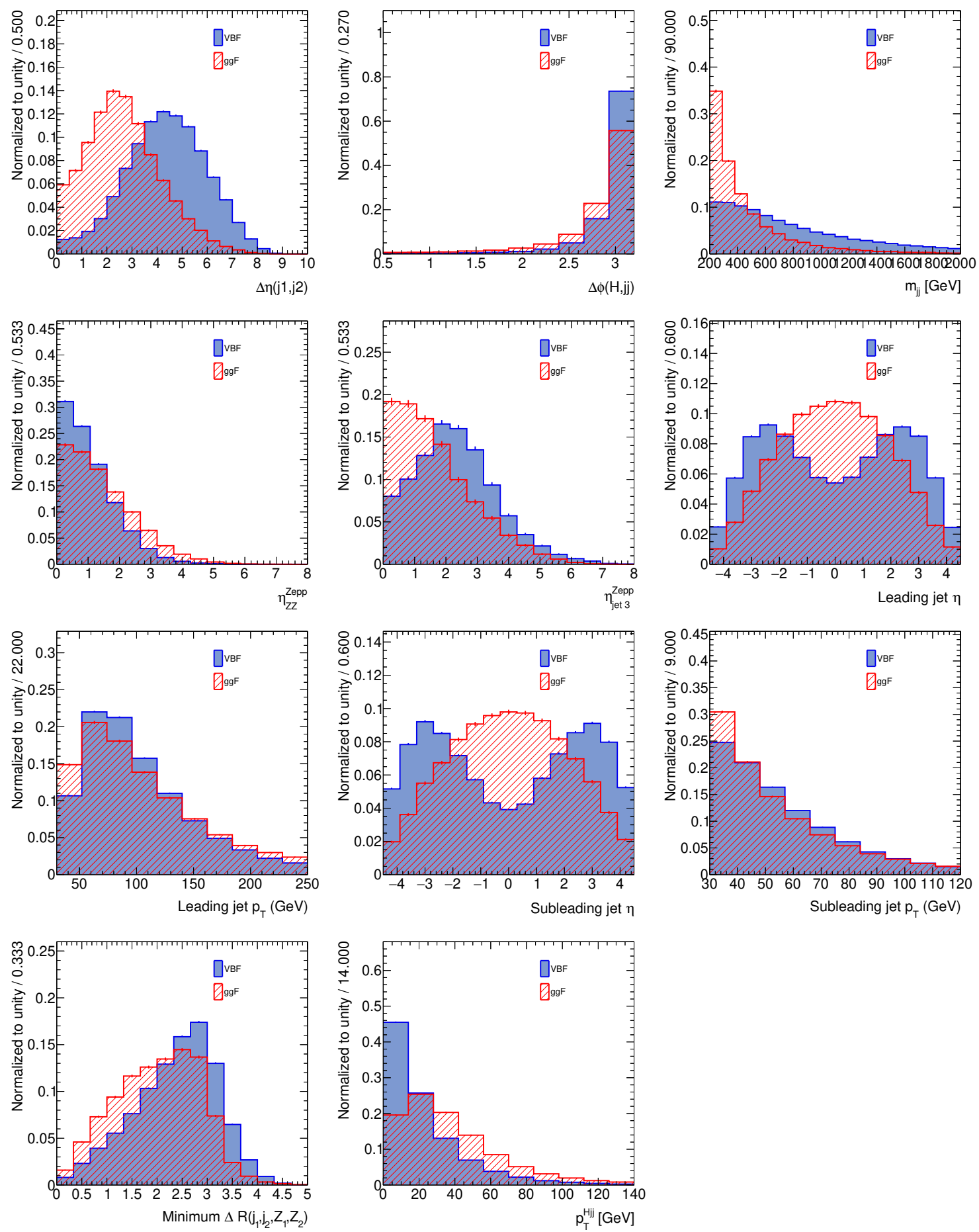

Figure 7.4: Discriminating variables considered for use in training the BDT to separate VBF and ggF production in the $H \rightarrow Z Z^{*} \rightarrow 4 \ell$ channel. 


\begin{tabular}{cccc}
\hline \hline Variable & Separation $\left\langle S^{2}\right\rangle$ & BDT importance & Importance ranking \\
\hline$\Delta \eta_{\mathrm{jj}}$ & 0.24 & 0.14 & 1 \\
$m_{\mathrm{jj}}$ & 0.23 & 0.097 & 4 \\
$p_{\mathrm{T}}^{H j j}$ & 0.11 & 0.087 & 6 \\
$\eta_{j_{2}}$ & 0.086 & 0.10 & 3 \\
$\eta_{j_{1}}$ & 0.053 & 0.067 & 11 \\
$\Delta \phi_{H j j}$ & 0.050 & 0.079 & 8 \\
$\Delta \mathrm{R}_{j Z}^{\min }$ & 0.046 & 0.084 & 7 \\
$\eta_{j_{3}}^{*}$ & 0.037 & 0.089 & 5 \\
$\eta_{Z Z}^{*}$ & 0.036 & 0.11 & 2 \\
$p_{\mathrm{T}}^{j_{1}}$ & 0.016 & 0.078 & 9 \\
$p_{\mathrm{T}}^{j_{2}}$ & 0.0095 & 0.073 & 10 \\
\hline
\end{tabular}

Table 7.1: Table of the shape separation $\left\langle S^{2}\right\rangle$ and BDT importance for a number of kinematic variables with power to separate VBF and ggF Higgs production. Variables are ranked in order of descending $\left\langle S^{2}\right\rangle$, with the corresponding rankings for BDT importance shown.

ninth in terms of separating power. The utility of this variable derives from its correlations with more separating variables, as its definition implicity carries information about the presence of additional radiation beyond the leading and subleading jets. In particular, tight cuts on $\eta_{Z Z}^{*}$ are correlated with a VBF-like phase space containing two high-rapidity jets and a central Higgs boson, as this limiting scenario corresponds to $\eta_{Z Z}^{*} \rightarrow 0$. This behaviour demonstrates how the shape separation and importance are complimentary metrics, and that an optimized BDT will rely on training variables which are both intrinsically separating, and which embed higher-order information through correlations with other variables.

\subsubsection{Optimization of input variables}

Given the conclusions of the previous section, it is worth investigating whether a subset of the full list of variables can be derived to optimize the overall performance 
of the BDT. In principle, the set of training variables chosen should be that which maximizes efficacy and efficiency, in the sense that a highly performant discriminant should be developed, trained using as few variables as possible to avoid "black box" scenarios where the BDT makes decisions that cannot be easily physically intuited.

In order to determine the optimal set of input training variables, the choice was made to perform an iterative scan over each possible permutation of the training variables shown in Figure 7.4 to find the configuration which provides the most effective discrimination with the least number of variables. That is to say, given the initial full list of $n=11$ training variables, the following steps were performed:

1. For $k$ training variables, iterate over all $n ! / k !(n-k)$ ! possible permutations

2. For each iteration $j$, perform a BDT training using the chosen input variables

3. Assess the maximal VBF signal significance with respect to ggF for the $j^{\text {th }}$ iteration

4. Determine the iteration which fully maximizes the VBF signal significance out of all $n ! / k !(n-k) !$ permutations

In lieu of performing a computationally-expensive signal significance calculation using e.g. a likelihood fit-based approach [131], this quantity is approximated using the median significance,

$$
Z_{0} \approx \sqrt{2\left((s+b) \ln \left(\frac{s+b}{b}\right)-s\right)}
$$

which is an asymptotic expression for the commonly-used log-likelihood ratio-based test statistic [131], valid when the number of background events, $b$, is large with 
respect to the number of signal events, $s$. The median significance is calculated using an integrated luminosity of $\int \mathcal{L}=1 \mathrm{fb}^{-1}$ for simplicity, as in practice, the median significance will scale with the square root of the integrated luminosity.

Within each iteration of step (3) defined above, a second scan procedure is performed to determine an optimal cut to place on the trained BDT discriminant. An iterative series of cuts in steps of 0.05 are placed on the BDT score, and at each step, the VBF signal significance is recomputed for events passing the cut. The chosen discriminant cut is that which maximizes the median significance for a given training iteration. The purpose of this procedure is to ensure that the performance of each training permutation is fairly assessed, as in principle measurements will be made in bins of the BDT score, with the most "VBF-enriched" bin providing the largest contribution to the overall significance. Accordingly, calculating the median significance only in the region above the discriminant threshold provides a straightforward approximation of the behaviour expected in the final analysis.

The optimal configuration of training variables for each choice of $k$ variables from $k=2$ to 11 is shown in Table 7.2, along with the optimal discriminant cut, and the corresponding median significance. As a baseline for the optimization, results are also shown for a training made with the variables used to build the Run-I BDT discriminant - namely, $m_{\mathrm{jj}}, \Delta \eta_{\mathrm{j} j}, p_{\mathrm{T}}^{j_{1}}, p_{\mathrm{T}}^{j_{2}}$, and $\eta_{j_{1}}$. It is evident that the change in efficacy of the BDT grows asymptotically small as more variables are added; while the change in optimal significance from $k=2$ to $k=6$ is $\sim 17 \%$, the change in significance from $k=6$ to $k=11$ is less than $2 \%$. Furthermore, the following variables all appear repeatedly among the optimal variable list permutations: 


\begin{tabular}{clcc}
\hline \hline$k$ & Optimal variable set & Optimal significance & Optimal BDT cut \\
\hline 2 & $m_{\mathrm{jj}}, \Delta \eta_{\mathrm{jj}}$ & 0.373 & 0.35 \\
3 & $m_{\mathrm{jj}}, \Delta \eta_{\mathrm{jj}}, p_{\mathrm{T}}^{H j j}$ & 0.419 & 0.5 \\
4 & $m_{\mathrm{jj}}, \Delta \eta_{\mathrm{jj}}, p_{\mathrm{T}}^{H}, \eta_{Z Z}^{*}$ & 0.430 & 0.5 \\
5 & $m_{\mathrm{jj}}, \Delta \eta_{\mathrm{jj}}, p_{\mathrm{T}}^{H j j}, \eta_{Z Z}^{*}, \eta_{j_{3}}^{*}$ & 0.434 & 0.5 \\
6 & $m_{\mathrm{jj}}, \Delta \eta_{\mathrm{jj}}, p_{\mathrm{T}}^{H j j}, \eta_{Z Z}^{*}, \eta_{j_{3}}^{*}, p_{\mathrm{T}}^{j_{2}}$ & 0.437 & 0.5 \\
7 & $($ Same as 6$)+p_{\mathrm{T}}^{j_{1}}$ & 0.440 & 0.6 \\
8 & $($ Same as 7$)+\Delta \mathrm{R}_{j Z}^{\min }$ & 0.443 & 0.6 \\
9 & $($ Same as 8$)+\Delta \phi_{H j j}$ & 0.444 & 0.55 \\
10 & $m_{\mathrm{jj}}, \Delta \eta_{\mathrm{jj}}, \eta_{j_{1}}, \eta_{j_{2}}, p_{\mathrm{T}}^{H j j}, \Delta \mathrm{R}_{j Z}^{\min }$, & 0.443 & 0.6 \\
& $\eta_{Z Z}^{*}, \eta_{j_{3}}^{*}, p_{\mathrm{T}}^{j_{2}}, \Delta \phi_{H j j}$ & & 0.6 \\
\hline Run-I variables & $(\mathrm{All}$ variables $)$ & 0.445 & 0.0 \\
\hline \hline
\end{tabular}

Table 7.2: Optimal training variable configurations for each choice of $k$ variables from the full set of $n=11$ possible variables.

$$
m_{\mathrm{jj}}, \Delta \eta_{\mathrm{jj}}, p_{\mathrm{T}}^{H j j}, p_{\mathrm{T}}^{j_{2}}, p_{\mathrm{T}}^{j_{1}}, \eta_{Z Z}^{*}, \Delta \mathrm{R}_{j Z}^{\min }
$$

In a sense, the configuration of variables shown above should not come as a surprise. As previously mentioned, both $m_{\mathrm{jj}}$ and $p_{\mathrm{T}}^{H j j}$ strongly characterize the VBF production process, along with the dijet pseudorapidity separation $\Delta \eta_{\mathrm{jj}}$, which is highly correlated to $m_{\mathrm{jj}}$. Furthermore, the distributions of the leading and subleading jet $p_{\mathrm{T}}$ differ enough between $\mathrm{VBF}$ and ggF production to provide some additional discriminating power. Lastly, the distributions of $\eta_{Z Z}^{*}$ and $\Delta \mathrm{R}_{j Z}^{\min }$ can indicate the presence of additional radiation in the central region of the detector, but do not explicitly act as vetoes with tight cuts.

While the variable $\eta_{j_{3}}^{*}$ does also reappear a few times in the optimal variable lists, tests done using simulated high-luminosity conditions (described in Appendix D) demonstrated that the variable held no discriminating power at high levels of pile-up. 


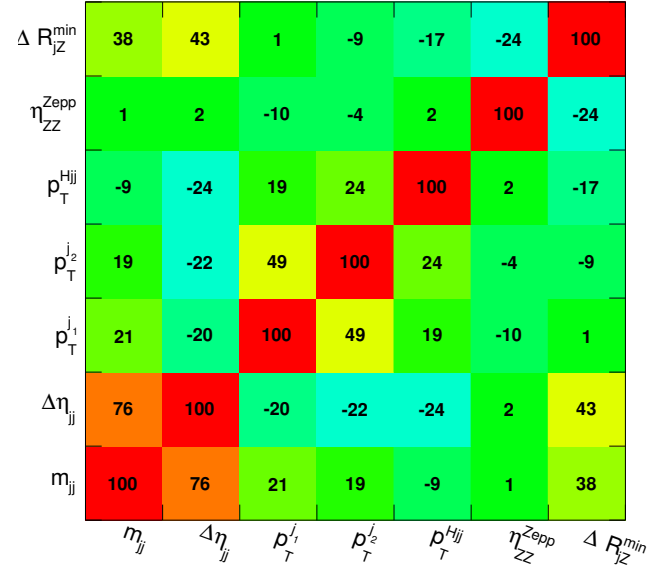

(a) LCCs (VBF)

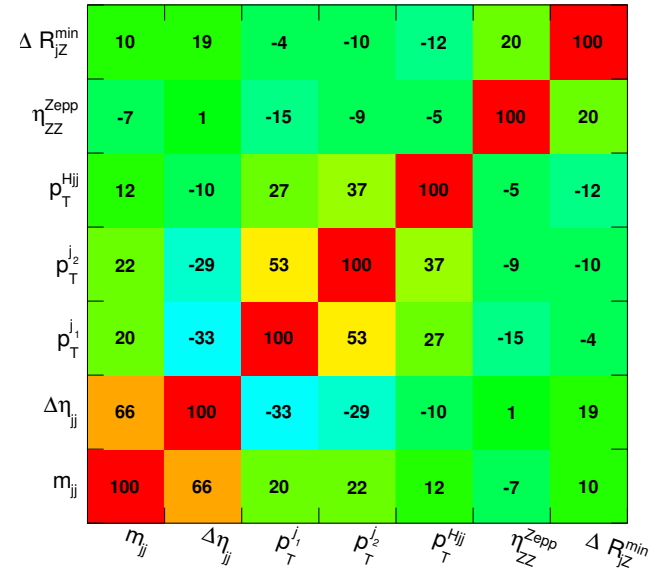

(b) LCCs $(\mathrm{ggF})$

Figure 7.5: Linear correlation coefficients for the optimized list of training variables, produced using truth-level samples of $\mathrm{VBF}$ and $\mathrm{ggF} H \rightarrow 4 \ell$ events, generated at $\sqrt{s}=14 \mathrm{TeV}$ using the POWHEG generator.

Furthermore, the modelling of the third subleading jet in $\mathrm{ggF}$ is highly generatordependent; while the use of an NLO QCD calculation provides a more accurate result derived from the matrix element calculation, a LO implementation would necessitate the modelling of the third jet through the SMC generator, which can lead to poorly modelled behaviour if the jet is not soft. Therefore, $\eta_{j_{3}}^{*}$ was removed from consideration to reduce the overall number training variables used in the BDT.

Matrices of the linear correlation coefficients (LCCs) for the remaining discriminating variables are found in Figure 7.5. The strong correlation between $m_{\mathrm{jj}}$ and $\Delta \eta_{\mathrm{jj}}$ is evident in the matrices corresponding to both VBF and ggF production, although the degree of correlation differs between processes. Furthermore, the differences in correlation strengths for $p_{\mathrm{T}}^{H j j}$ and other training variables in VBF and ggF production may explain the consistent presence of these three variables among the lists of 
optimal permutations.

Given that the above list of variables consistently provides a superior discrimination of VBF and ggF production, with the addition of more variables bringing only marginal performance improvements, it was decided that only these seven variables would be used to perform the BDT training. Therefore, this configuration of variables fulfils the expectation of both maximal efficacy and efficiency in the BDT training. Any further tests or modifications to the training methodology would be done using samples of reconstruction-level events, as the physics-motivated aims of the optimization were fulfilled, with the remaining aims targeted towards optimizing experimental signal efficiency and purity, while minimizing measurement uncertainties.

\subsection{BDTs and theory uncertainties in $H+2 j$ events}

One important consideration when training a discriminant to separate physical processes is whether the signal and background process cross sections are well-modelled in all regions of phase space probed by the discriminant. As previously mentioned in Section 2.5, higher-order QCD corrections to the ggF cross section calculation are highly non-trivial, and encompass both virtual and real corrections to the tree-level diagram. These corrections lead to theoretical complications when cuts are placed which restrict the number of final state jets in $\mathrm{ggF}$, such as tight cuts on $p_{\mathrm{T}}^{H j j}$ which create two-jet-enriched regions reminiscent of tree-level VBF Higgs production.

In general, the calculation of an observable (e.g. a cross section) in perturbative QCD can be written as,

$$
O=\phi \otimes \hat{O}+P_{O}
$$




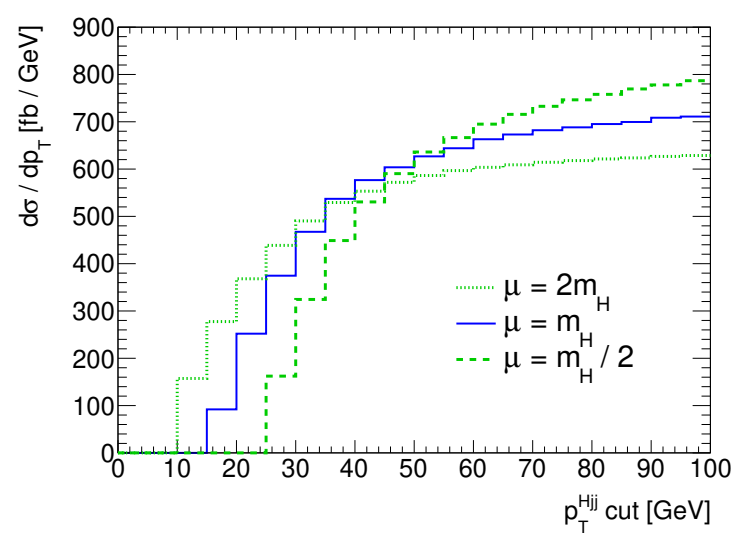

Figure 7.6: Effect of cuts on $p_{\mathrm{T}}^{H j j}$ on the parton-level ggF $H+2 j$ differential cross section $d \sigma / d p_{\mathrm{T}}$, along with the scale variation error bands defined by $\mu_{\mathrm{R}}=\mu_{\mathrm{F}}=m_{H} / 2$ and $2 m_{H}$. All distributions are derived using MCFM with $m_{H}=125 \mathrm{GeV}$, requiring jet $p_{\mathrm{T}}$ above $30 \mathrm{GeV}$, and $m_{\mathrm{jj}}>120 \mathrm{GeV}$.

where $\hat{O}=\sum_{n} c_{n} \alpha_{s}^{n}$ is the partonic version of the observable, with the series calculated to some finite order, and $\phi$ represents the parton distribution / fragmentation functions. The terms in $P_{O}$ represent corrections of the form $p_{n}\left(\Lambda_{\mathrm{QCD}} / Q\right)$, where $Q$ is the energy scale of the process, and $\Lambda_{\mathrm{QCD}}$ is the QCD energy scale. For ggF cross sections with $\geq 1$ final state jets, the imposition of cuts which suppress Feynman diagrams featuring the real emission of soft (or "infrared / IR") gluons leads to the presence of large Sudakov logarithms in $c_{n}$ at each order of the perturbative expansion $\hat{O}$. For example, placing an exclusive cut on the jet transverse momentum $p_{\mathrm{T}}^{\text {cut }}$, such that events are separated into $N$ and $\geq N+1$-jet bins, induces Sudakov logarithms of the form $L=\ln \left(p_{\mathrm{T}}^{\text {cut }} / Q\right)$ [132]. For very tight cuts, the logarithms become $\mathcal{O}(1)$, and dominate the perturbative series, leading to "IR-sensitivity" in the cross section prediction. If the logarithms become large enough that they overcome the $\alpha_{s}$ suppression necessary for perturbative QCD (see Section 2.1), then the perturbative expansion completely breaks down, leading to meaningless cross section estimates [132].

In general, the use of fixed-order QCD calculations also comes with associated 
uncertainties due to the fact that there are higher-order corrections "missing" from the perturbative expansion. These uncertainties are typically estimated by varying the renormalization $\left(\mu_{\mathrm{R}}\right)$ and factorization $\left(\mu_{\mathrm{F}}\right)$ scales in the cross section calculation, often in a correlated fashion using a single scale $\mu=\mu_{\mathrm{F}}=\mu_{\mathrm{R}}=\mu_{0} / 2$ or $2 \mu_{0}$, where $\mu_{0}$ is the central scale choice (typically $m_{H}$ or $m_{H} / 2$ ). So, beyond the estimation of the cross section itself, the use of tight or exclusive cuts on real emissions poses difficulties for estimating the uncertainties resulting from missing higher-order QCD corrections, as well. An illustration of the effect on the scale variation uncertainties is shown in Figure 7.6 for a parton-level ggF $H+2 j$ calculation at NLO QCD, made using the MCFM Monte Carlo generator [133]. In this distribution, the $x$-axis shows the $p_{\mathrm{T}}^{H j j}$ cut that events are required to pass, and the $y$-axis shows the differential cross section $d \sigma / d p_{\mathrm{T}}$ and scale variation error bands for the region passing the cut.

For the case of an inclusive $\geq 2$-jet calculation, it is generally expected that the error band defined by the $\mu=\mu_{0} / 2$ scale choice should provide a continuous upper bound for the perturbative uncertainty, with $\mu=2 \mu_{0}$ providing the lower bound. However, Figure 7.6 shows the effects induced by large Sudakov logarithms when a "2-jet-enriched" phase space is created using tight cuts on $p_{\mathrm{T}}^{H j j}$. In particular, the scale variation uncertainties shrink from $\mathcal{O}(30 \%)$ to $\mathcal{O}(5 \%)$ in certain regions, and the lower and upper error bands cross over at roughly $p_{\mathrm{T}}^{H j j}<50 \mathrm{GeV}$.

In practice, these effects can be understood as events "migrating" back and forth between the 2 and $\geq 3$-jet-enriched bins in a manner dependent on the scale choice, as the renormalization and factorization scales also dictate the hardness of the additional radiation in the parton-level process. In particular, the effects of migration are anticorrelated with respect to scale choice, with a smaller scale choice driving migration 
from the 2-jet to $\geq 3$-jet bins, and a larger scale choice doing the opposite. Accordingly, any estimate of the perturbative QCD uncertainty in the "2-jet-enriched" region must incorporate not only cross section variation effects, but also migration effects as a consequence of the presence of Sudakov logarithms.

To this end, the Stewart-Tackmann (S-T) method can be employed to produce more sensible, symmetric perturbative uncertainties for the 2-jet-enriched cross section when placing cuts on IR-sensitive variables such as $p_{\mathrm{T}}^{H j j}$. The full implementation of the method is described in detail in Refs. [95] and [134]. The main conclusion of this formulation is that, when placing an exclusive cut on an IR-sensitive variable such as $p_{\mathrm{T}}^{H j j}$, the uncertainty on the cross section of the 2-jet-enriched phase space $\left(\Delta \sigma_{2}\right)$ can be estimated as,

$$
\Delta \sigma_{2}^{2}=\Delta \sigma_{\geq 2}^{2}+\Delta \sigma_{\geq 3}^{2}
$$

where $\Delta \sigma_{\geq 2}$ is the cross sectional uncertainty for the inclusive $\geq 2$-jet cross section, and $\Delta \sigma_{\geq 3}$ is the cross sectional uncertainty for the $\geq 3$-jet-enriched region, i.e. that which fails the cut on $p_{\mathrm{T}}^{H j j}$. To estimate these uncertainties, parton-level NLO QCD $H+2 j$ cross sections were computed using the MCFM Monte Carlo generator assuming $m_{H}=125 \mathrm{GeV}$, as well as requiring jet $p_{\mathrm{T}}$ above $30 \mathrm{GeV}$, and $m_{\mathrm{jj}}>120 \mathrm{GeV}$. Figure 7.7 shows the estimated S-T uncertainties as a function of the $p_{\mathrm{T}}^{H j j}$ cut threshold, with the corresponding relative uncertainties given in Table 7.3. It is clear that the S-T method produces much more sensible estimates of the scale variation uncertainty, as the error bands both continuously envelop the nominal cross section predictions, and for loose cuts on $p_{\mathrm{T}}^{H j j}$, they also converge to the error bands created by the $\mu_{0} / 2$ and $2 \mu_{0}$ scale variations. 


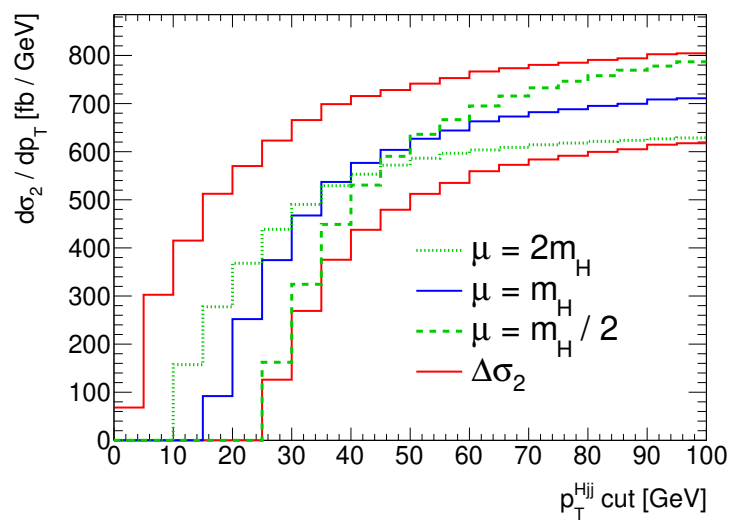

Figure 7.7: Effect of cuts on $p_{\mathrm{T}}^{H j j}$ on the parton-level ggF $H+2 j$ differential cross section $d \sigma / d p_{\mathrm{T}}$, along with the scale variation error bands defined by $\mu_{\mathrm{R}}=\mu_{\mathrm{F}}=m_{H} / 2$ and $2 m_{H}$. Also shown are the symmetrized error bands computed using the Stewart-Tackmann method. All distributions are derived using MCFM with $m_{H}=125 \mathrm{GeV}$, requiring jet $p_{\mathrm{T}}$ above $30 \mathrm{GeV}$, and $m_{\mathrm{jj}}>120 \mathrm{GeV}$.

\begin{tabular}{cc}
\hline \hline$p_{\mathrm{T}}^{H j j}$ cut $(\mathrm{GeV})$ & $\Delta \sigma_{2} / \sigma_{2}(\%)$ \\
\hline 20 & 126 \\
25 & 66.4 \\
30 & 42.4 \\
40 & 24.1 \\
50 & 18.3 \\
60 & 15.6 \\
70 & 14.4 \\
80 & 13.8 \\
\hline \hline
\end{tabular}

Table 7.3: Relative uncertainties for the 2-jet enriched region, $\Delta \sigma_{2} / \sigma_{2}$, in bins of rectangular cuts on $p_{\mathrm{T}}^{H j j}$, computed using the Stewart-Tackmann method. 


\subsection{Flattening the distribution of $p_{\mathrm{T}}^{H j j}$}

Given that $p_{\mathrm{T}}^{H j j}$ was included as a training variable in the BDT based on its ability to separate VBF and ggF Higgs production, it became crucial that the BDT was trained in a way that prevented cuts on $p_{\mathrm{T}}^{H j j}$ which could induce large theoretical uncertainties like those seen in Table 7.3. To prevent the BDT from making such cuts, the choice was made to use a modified version of the variable that is "flattened" below some threshold value, such that,

$$
p_{\mathrm{T}}^{\prime H j j}=\left\{\begin{array}{ll}
p_{\mathrm{T}}^{H j j} & \text { if } p_{\mathrm{T}}^{H j j}>X \mathrm{GeV} \\
X \mathrm{GeV} & \text { if } p_{\mathrm{T}}^{H j j}<X \mathrm{GeV}
\end{array} .\right.
$$

With this modification, a tight cut on the BDT classifier could induce a QCD scale variation uncertainty that is, at most, equivalent to the Stewart-Tackmann uncertainty for an exclusive cut on $p_{\mathrm{T}}^{H j j}$ at $X \mathrm{GeV}$. An example of the shape of $p_{\mathrm{T}}^{H j j}$ in ggF $H+2 j$ events, before and after "flattening" the distribution, is given in Figure 7.8 for a threshold value of $30 \mathrm{GeV}$.

In order to balance the discriminating power of the BDT with the associated perturbative uncertainty size, an iterative scan was performed to select the optimal flattening threshold for $p_{\mathrm{T}}^{\prime H j}$. The scan proceeded through nine iterations, with the threshold incrementing in $10 \mathrm{GeV}$ steps between values of $20 \mathrm{GeV}$ and $100 \mathrm{GeV}^{1}$. At the $i^{\text {th }}$ iteration, the distribution of $p_{\mathrm{T}}^{H j j}$ was "flattened" below $X_{i} \mathrm{GeV}$, and the BDT was re-trained with the newly-modified $p_{\mathrm{T}}^{\prime H j j}$ in place of $p_{\mathrm{T}}^{H j j}$.

Considering the relatively smaller number of iterations with respect to the scan

\footnotetext{
${ }^{1}$ No thresholds below $20 \mathrm{GeV}$ were tested, as beyond this point, the parton-level $H+2 j$ cross section was found to be negative.
} 


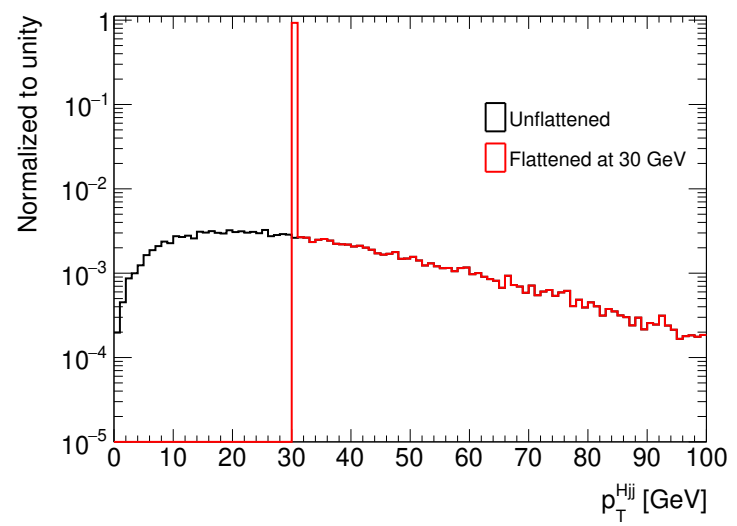

Figure 7.8: A comparison of the shape of $p_{\mathrm{T}}^{H j j}$ in ggF $H+2 j$ events, before and after "flattening" the distribution to create $p_{\mathrm{T}}^{\prime H j j}$, for a threshold value of $30 \mathrm{GeV}$.

done in Section 7.3.1, this optimization scan could employ a full likelihood-based fit, implemented using the Root-based HistFactory [135] and RooStats [136] statistical analysis software. In particular, a negative log-likelihood minimization technique [131] was applied across 10 bins of the BDT discriminant between $[-1,1]$, where the expected SM signal event yields in each bin were used as pseudo-data. The log-likelihood method tests two hypotheses: the nominal case of a VBF "signal" present on top of the ggF "background"; and a "background-only" hypothesis where the VBF signal strength is zero, with the excess yield assumed to be caused by a change in the dijet ggF cross section. The irreducible $q q Z Z$ process was also used as a fixed background source to better emulate real experimental conditions.

The primary metric of improvement in this scan was the VBF signal significance $Z_{0}^{\mathrm{VBF}}$ over the ggF and $q q Z Z$ yields, which was computed using the background-only $p$-value $\left(p_{0}\right)$, derived from the test statistic $q_{\mu}$,

$$
q_{\mu}=-2 \ln \frac{\mathcal{L}\left(\mu_{\mathrm{VBF}}, \theta\right)}{\mathcal{L}\left(\hat{\mu}_{\mathrm{VBF}}, \hat{\theta}\right)}
$$


such that $q_{0}$ represents the background-only hypothesis with $\mu_{\mathrm{VBF}}=0$. Here, $\mu_{\mathrm{VBF}}$ is the VBF signal strength parameter $\mu_{\mathrm{VBF}}=\sigma_{\mathrm{VBF}} / \sigma_{\mathrm{VBF}, \mathrm{SM}}$, while $\theta$ represents the set of nuisance parameters for signal and background, with the nuisance parameters constrained using auxiliary measurement terms. The terms with single circumflexes ( $\hat{\mu}$ and $\hat{\theta}$ ) denote the unconditional maximum likelihood estimates of each parameter, as explained further in Section 9.2. The value of $p_{0}$ is defined to be the probability to obtain a value of $q_{0}$ larger than the observed value under the background-only hypothesis. In particular, the value of $p_{0}$ can be expressed as,

$$
p_{0}=\int_{q_{0, \mathrm{obs}}}^{\infty} f\left(q_{0} \mid 0, \hat{\theta}_{0}\right) d q_{0}
$$

where the test statistic shape $f\left(q_{0} \mid 0, \hat{\theta}_{0}\right)$ is represented by a $\chi^{2}$ distribution with one degree of freedom. The $p_{0}$ value is converted to the corresponding number of standard deviations $(\sigma)$ in a one-sided Gaussian test, yielding the VBF significance $Z_{0}^{\mathrm{VBF}}$.

The error on the signal strength $\Delta \mu_{\mathrm{VBF}}$ was also used as a metric of improvement, as in practice, one always retains $\mu_{\mathrm{VBF}}=1$ from the log-likelihood fit when not testing the background-only hypothesis. So, the relevant concern is to optimize the VBF signal strength measurement precision by minimizing the size of $\Delta \mu_{\mathrm{VBF}}$, if possible. To limit the effects of statistical uncertainty in the likelihood fit, the likelihood fits were made assuming an integrated luminosity of $\mathcal{I}=100 \mathrm{fb}^{-1}$, which is roughly the amount of data recorded by the LHC up to the end of 2017 .

In general, performing a cut on a multivariate discriminant produces cuts on the constituent training variables whose shapes are defined by non-linear equations (as opposed to traditional rectangular cuts). As it pertains to IR-sensitive variables such as $p_{\mathrm{T}}^{H j j}$, placing a loose cut on the BDT score should not induce the same level 
of uncertainty as a tight cut, as the phase space isolated by each cut will contain different fractions of exclusively 2 -jet and $\geq 2$-jet events. To account for this effect in the iterative scan, the effective ggF perturbative uncertainty in a given BDT score bin was computed using a weighted average of S-T uncertainties for different exclusive cuts on $p_{\mathrm{T}}^{H j j}$. In particular, the relative uncertainty on the ggF yield in a given BDT bin was calculated as,

$$
\left(\frac{\Delta \sigma_{2}^{\text {tot }}}{\sigma_{2}}\right)^{2}=\sum_{i=1}^{N}\left(\frac{\Delta \sigma_{2}^{i}}{\sigma_{2}^{j}} \times h\left(x_{i}\right)\right)^{2}
$$

Here, $i$ is the histogram bin index, $h$ is an $N$-bin probability density histogram of $p_{\mathrm{T}}^{H j j}$ built from events in the specified BDT score range, and $x_{i}$ is the central value of the $i^{\text {th }}$ histogram bin. In addition, $\sigma_{2}^{i}$ is the 2-jet-enriched cross section for events passing a cut $p_{\mathrm{T}}^{H j j}<x_{i}$, and $\Delta \sigma_{2}^{i}$ is the corresponding S-T uncertainty for such a cut, with both values being derived from samples generated with MCFM, as described above. The above weighted sum operates as follows: In the limit that the BDT score cut induces tight restrictions on $p_{\mathrm{T}}^{H j j}$, the shape of the $p_{\mathrm{T}}^{H j j}$ histogram will approach a delta function at the flattening threshold, hence the effective uncertainty will approach the S-T uncertainty for a rectangular cut at said threshold. Conversely, when the BDT score range of interest produces only loose restrictions on $p_{\mathrm{T}}^{H j j}$, the total uncertainty will shrink as the bins with high $p_{\mathrm{T}}^{H j j}$ are more populated.

The intention of this scan was to find a threshold for flattening $p_{\mathrm{T}}^{H j j}$ which maximized $Z_{0}^{\mathrm{VBF}}$, while limiting the effect that the (potentially large) perturbative uncertainty has on $\Delta \mu_{\mathrm{VBF}}$. The resultant distribution of the expected VBF signal significance at each threshold choice is shown in Figure 7.9, along with the expected signal strength uncertainty, $\Delta \mu_{\mathrm{VBF}}$. Also shown on these plots are the expected $Z_{0}^{\mathrm{VBF}}$ 

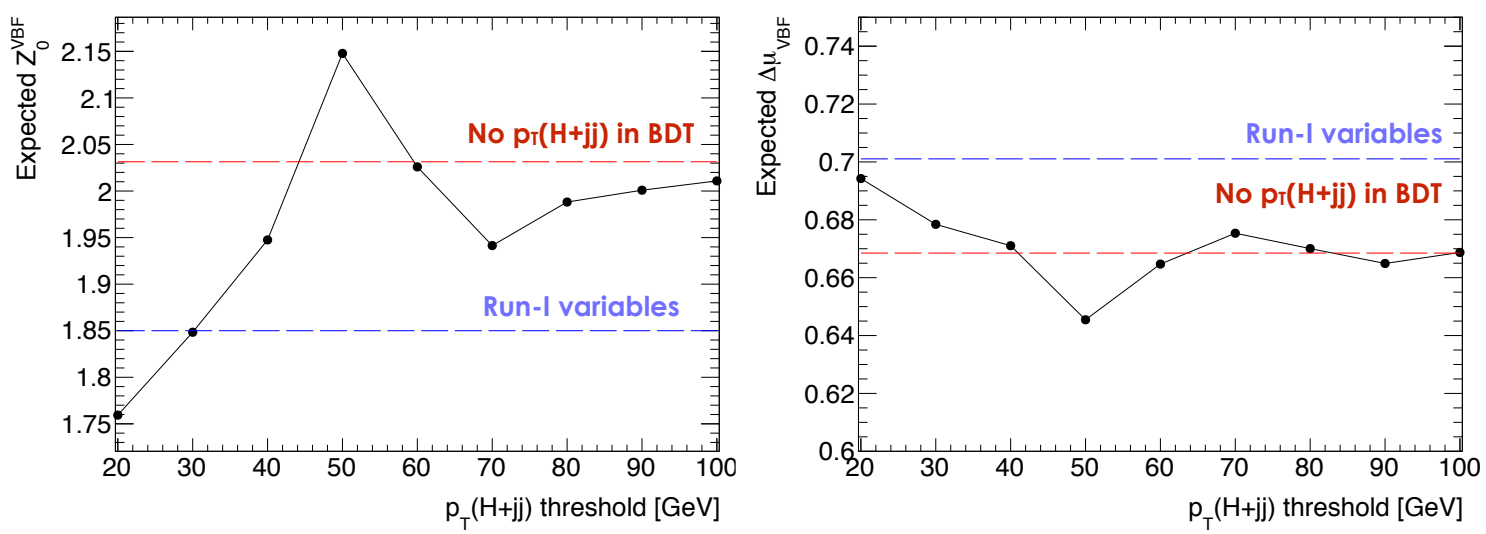

Figure 7.9: Distribution of expected VBF significance (left) and signal strength uncertainty (right) using a likelihood fit across 10 BDT bins in $[-1,1]$, under different flattening thresholds of $p_{\mathrm{T}}^{H j j}$. Also shown are analogous values for BDTs trained using the Run-I variables (blue line), and a BDT trained without $p_{\mathrm{T}}^{H j j}$ (red line).

and $\Delta \mu_{\mathrm{VBF}}$ for two alternate scenarios: a BDT trained using the list of "optimized" variables derived in Section 7.3.1, minus $p_{\mathrm{T}}^{H j j}$; and the same variables used to train the Run-I BDT discriminant.

It was found that $Z_{0}^{\mathrm{VBF}}$ was maximized using a threshold value of $50 \mathrm{GeV}$, indicating that a balance of discriminating power and uncertainty size was reached. For thresholds below this point, the presence of larger S-T uncertainties for lower $p_{\mathrm{T}}^{H j j}$ allows the ggF component to progressively "absorb" the VBF contribution, shrinking the signal significance, and increasing the signal strength uncertainty. Conversely, higher thresholds reduce the utility of $p_{\mathrm{T}}^{H j j}$, reducing it effectively to a delta function, such that it no longer has power to discriminate VBF and ggF production. Consequently, the variable is used successively less often to make decisions in the BDT. In this limit, the results asymptotically reach a point which is equivalent to a BDT training made without the use of $p_{\mathrm{T}}^{H j j}$, as one might expect.

However, naively binning this variable below the given threshold may not totally 


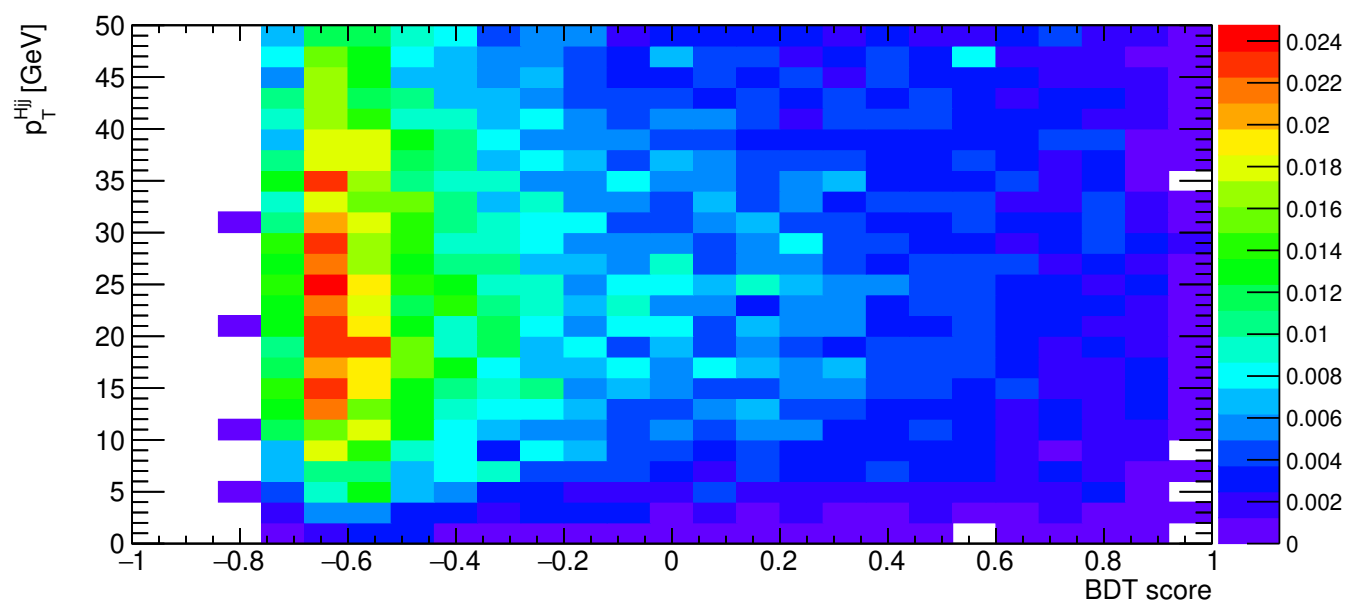

Figure 7.10: Distribution of $p_{\mathrm{T}}^{H j j}$ vs. BDT score in $\operatorname{ggF} H \rightarrow Z Z^{*} \rightarrow 4 \ell$ events with $m_{\mathrm{jj}}>$ $120 \mathrm{GeV}$ and $p_{\mathrm{T}}^{H j j}<50 \mathrm{GeV}$, indicating that the trained BDT does not have a visible correlation with $p_{\mathrm{T}}^{H j j}$ in this region of phase space.

eliminate this problematic behaviour, as correlations with other training variables may still induce implicit cuts on $p_{\mathrm{T}}^{H j j}$. To validate that the BDT is not entering this problematic region of phase space, the shape of $p_{\mathrm{T}}^{H j j}$ below $50 \mathrm{GeV}$ was plotted against the full range of the BDT score, as shown in Figure 7.10. If the classifier is sculpting the distribution of $p_{\mathrm{T}}^{H j j}$ to favour small $p_{\mathrm{T}}$ values at high BDT scores, then one would expect the 2D histogram of $p_{\mathrm{T}}^{H j j}$ vs. BDT score to show a distinct anticorrelation. However, it is clear from this distribution that no meaningful correlation is observed between the two variables. Therefore, the BDT does not induce cuts in the "IR-sensitive" region of the $p_{\mathrm{T}}^{H j j}$ phase space, avoiding the induction of large theoretical uncertainties. 


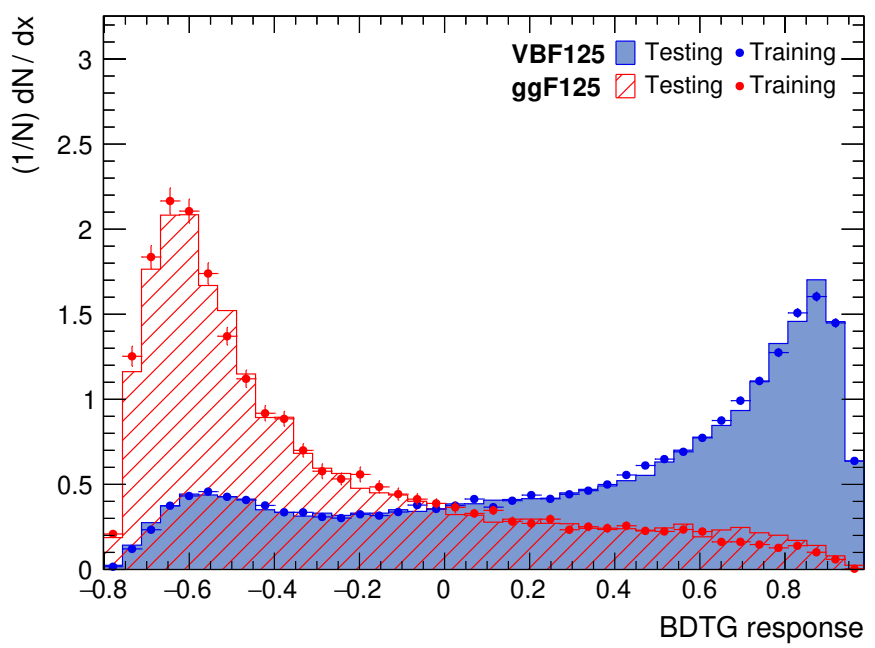

Figure 7.11: Superimposition of the BDT response for the training and testing samples of VBF and $\operatorname{ggF} H \rightarrow 4 \ell$ events.

\subsection{Overtraining and bias tests}

Another consideration when training a BDT is that it should not bias or "sculpt" any parameter of interest in the analysis - for example, the invariant mass of the four lepton system $m_{4 \ell}$, which is reconstructed as the Higgs boson mass. Furthermore, the classifier should be robust enough that it does not favour training events which are not properly represented in the testing samples - a behaviour known as "overtraining".

Superimposed distributions of the BDT response for the training and testing samples are shown in Figure 7.11 for both signal and background events. It is evident that there is good agreement in the discriminant shapes of the training and testing samples for both signal and background, allowing for some fluctuations due to the finite training sample sizes. To check that no sculpting or bias was induced on the shape or peak position of $m_{4 \ell}$ through use of the BDT training, distributions of the constrained mass spectra for $\mathrm{VBF}$ and $\mathrm{ggF} H \rightarrow Z Z^{*} \rightarrow 4 \ell$ events were compared in different 


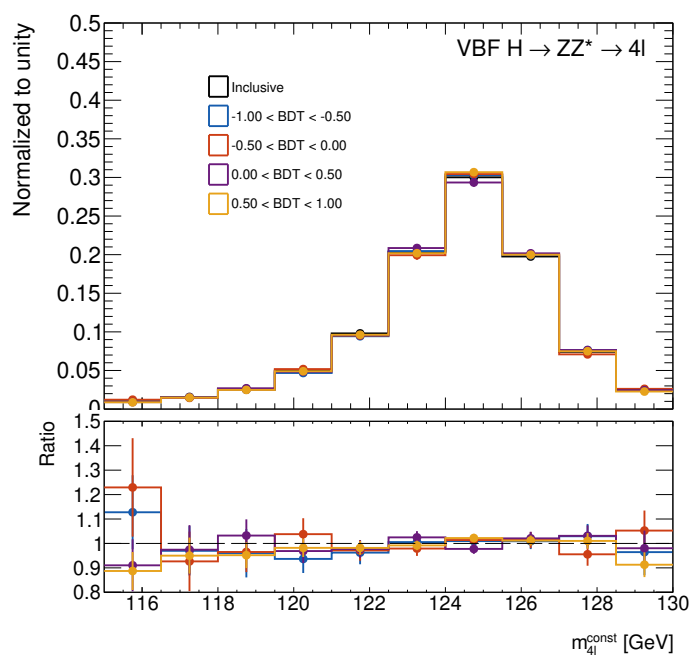

(a) $\operatorname{VBF} H \rightarrow Z Z^{*} \rightarrow 4 \ell$

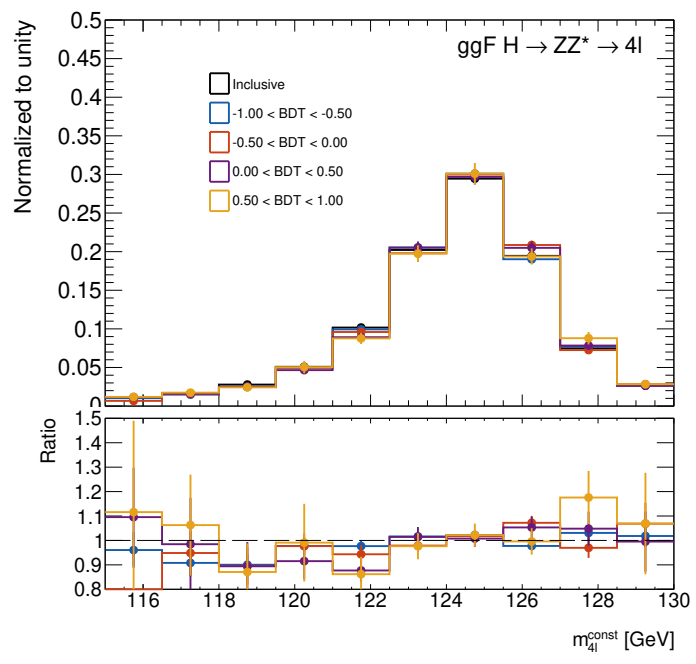

(b) $\operatorname{ggF} H \rightarrow Z Z^{*} \rightarrow 4 \ell$

Figure 7.12: Shape of $m_{4 \ell}$ in different regions of the BDT discriminant for both VBF (left) and ggF (right) production.

regions of the BDT score. In the ideal scenario where there is no correlation between the BDT discriminant and $m_{4 \ell}$, the shapes of the mass distributions in each region should be approximately identical, allowing for bin-by-bin fluctuations in regions of limited event yields. The resultant plots of $m_{4 \ell}$ are shown in Figure 7.12 for each production type, in BDT score regions delineated by bin edges of $[-1,-0.5,0,0.5,1]$. Ultimately, it was found that there is no meaningful effect on the shape of the $m_{4 \ell}$ spectrum from placing cuts on the BDT score.

One can also compare the LCCs between training variables at reconstructionlevel for the VBF and ggF processes, to ensure that the conclusions found from the truth-level discriminating variable optimization carry over once detector effects are factored in. While the magnitudes of the correlations will differ, owing particularly to reconstruction inefficiencies and the presence of pile-up jets, the relative degrees and signs of the correlations are consistent in each training with the findings at truth-level. 


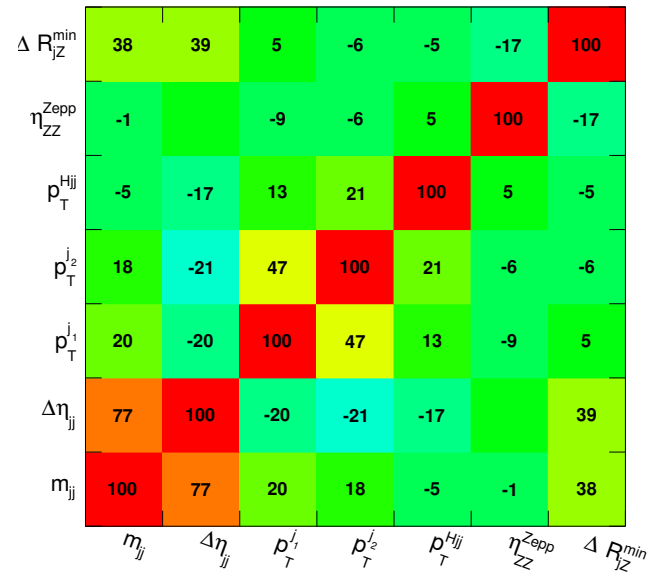

(a) LCCs (VBF)

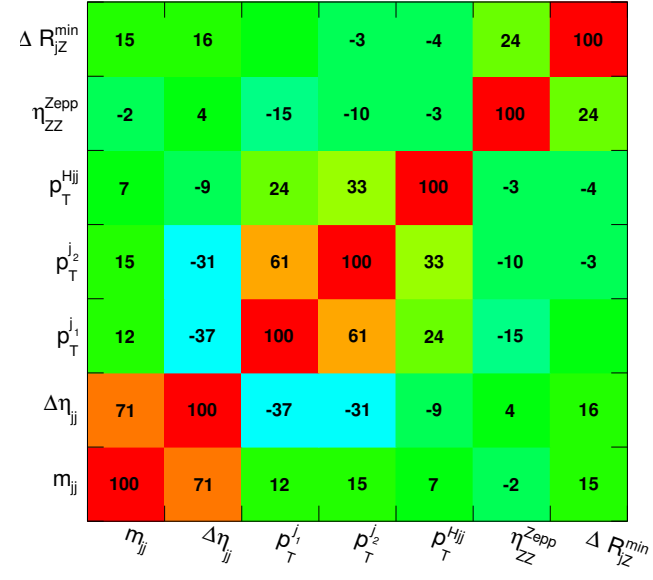

(b) LCCs (ggF)

Figure 7.13: Linear correlation coefficients for the BDT training produced using fully-simulated samples of $\mathrm{VBF}$ and $\mathrm{ggF} H \rightarrow 4 \ell$ events.

Worth noting is that the Powheg NnLOPS sample used to model ggF production is LO for $\geq 2$-jet events, so the agreement in the correlation structure between the reconstruction-level NNLOPS sample and the truth-level, NLO QCD $H+2 j$ sample (generated via the Powheg HJJ generator) indicates a good degree of robustness in the choice of training variables.

\subsection{Discriminants for other categories}

Although the focus of this chapter was on the VBF $H \rightarrow Z Z^{*} \rightarrow 4 \ell$ BDT discriminant, the statistical analysis methodology in the $H \rightarrow Z Z^{*} \rightarrow 4 \ell$ channel also relies on additional observables built from multivariate discriminants which are sensitive to the different SM Higgs boson production mechanisms, and applied in categories delineated by exclusive jet bins. The discriminants are used either to separate 
Higgs signal from the irreducible $q q Z Z$ background, or to distinguish Higgs boson production modes in categories with small experimental backgrounds. Along with the classifier described in this chapter, the following discriminants are used:

- 0-jet category: A BDT is trained to separate ggF production from $q q Z Z$ background

- 1-jet, low and medium $p_{\mathbf{T}}^{H}$ categories: A BDT is trained to separate VBF Higgs signal from background + non-VBF Higgs signal

- $\geq$ 2-jet, VH-hadronic-enriched category: A BDT is trained to separate hadronic VH Higgs signal from background + non-hadronic VH Higgs signal

The 0-jet discriminant is trained using $p_{\mathrm{T}}^{H}$ and $\eta_{H}$, as well as a kinematic discriminant built from LO matrix element calculations of the $q q Z Z$ and ggF processes. The discriminant for the VH-enriched category is trained using the same input variables as the VBF discriminant, with the exception of $p_{\mathrm{T}}^{H j j}$. Lastly, the 1-jet discriminant for the low and medium $p_{\mathrm{T}}^{H}$ categories are built from the leading jet $p_{\mathrm{T}}$ and pseudorapidity, as well as $\Delta R$ between the leading jet and $Z Z^{*}$ system.

For each signal process, one-dimensional, binned probability density functions are constructed using the BDT discriminant shapes in each analysis category. Event counting is used in the 1-jet category with $p_{\mathrm{T}}^{H}>120 \mathrm{GeV}$, as well as the VBFenriched category with $p_{\mathrm{T}}^{j_{1}}>200 \mathrm{GeV}$, and the leptonic VH-enriched and ttH-enriched categories. To form the PDF in the 0-jet category, 15 bins with even bin width are used spanning a range from -1 to 1 . In all the other categories, 10 bins with even bin width are used. The shapes of the per-category discriminants are shown in Figure 7.14 for each Higgs boson production mode used in the various trainings. 

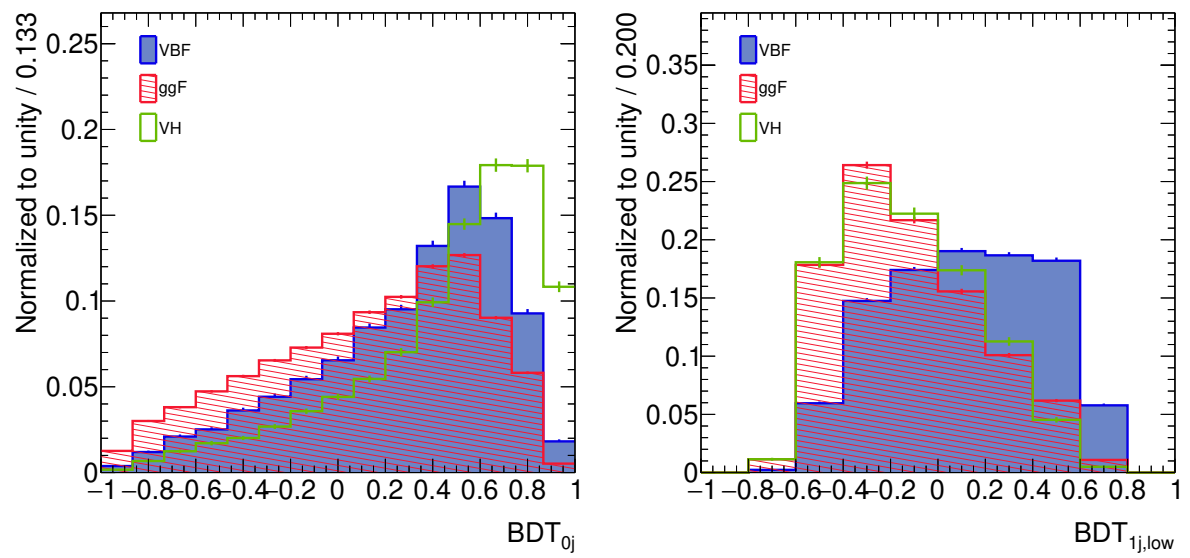

(a) 0 -jet

(b) 1 -jet, $p_{\mathrm{T}}^{H}<60 \mathrm{GeV}$
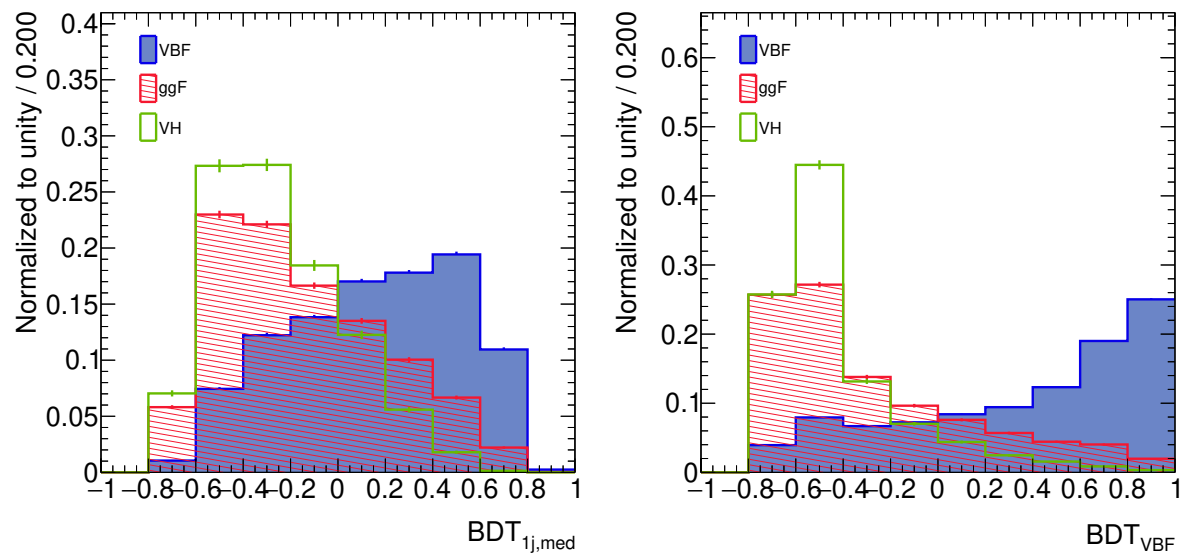

(c) 1 -jet, $60<p_{\mathrm{T}}^{H}<120 \mathrm{GeV}$

(d) $\geq 2$-jet, VBF-enriched

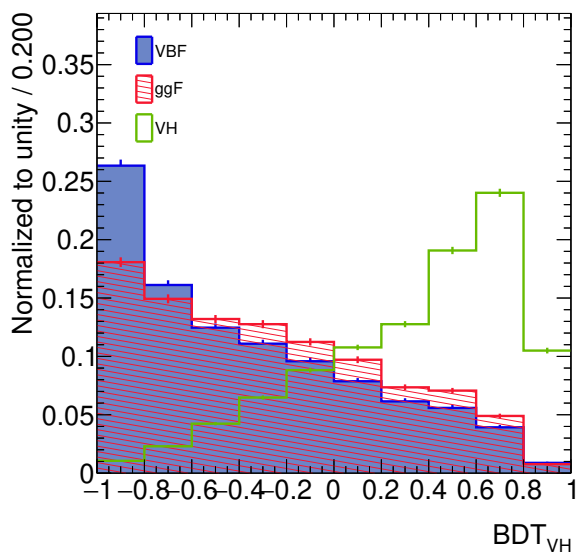

(e) $\geq 2$-jet, VH-enriched

Figure 7.14: Per-category discriminants used in the $H \rightarrow Z Z^{*} \rightarrow 4 \ell$ analysis, shown for each major considered Higgs boson production mode. 


\section{Chapter 8}

\section{Systematic uncertainties}

A careful evaluation of potential sources of systematic uncertainty is crucial for any physics analysis. Broadly speaking, systematic uncertainties fall under two categories: experimental uncertainties, which are derived from limitations in modelling detector resolution, object reconstruction efficiency, and energy calibration; and theoretical uncertainties, which arise from uncertainties on the theoretical calculations used to make signal and background predictions. The author's contributions to these aspects of the analysis were focused on the derivation and implementation of theoretical uncertainty estimates for ggF-mediated Higgs boson production. Emphasis is given to the uncertainties induced by the division of $\mathrm{ggF}$ events into exclusive jet bins, whose estimation must necessarily factor in the current limitations of perturbative QCD to treat such a configuration. 


\subsection{Experimental uncertainties}

Any experimental result in collider physics will invariably be subject to uncertainties introduced by inefficiencies in the detection and reconstruction of particles, as well as the calibration of their reconstructed energies. In the context of the $H \rightarrow Z Z^{*} \rightarrow 4 \ell$ analysis, the dominant experimental uncertainties emerge from reconstruction efficiency effects in electrons, muons, and jets, as well as the calibration of each object.

Independent of the reconstruction, the recorded integrated luminosity has an associated uncertainty, which for the combined $2015+2016$ ATLAS data set is $3.2 \%$. The uncertainty is derived using a preliminary calibration of the luminosity scale derived from $x-y$ scans of the beam-separation performed in August 2015 and May 2016 [137]. The use of pile-up modelling also induces an associated uncertainty on the predicted signal and background yields. By varying the average number of pile-up events in the simulation, an uncertainty is derived which covers the variation in the ratio of the predicted inelastic cross sections to their corresponding measured values [138]. The uncertainty due to pile-up modelling is found to be $\sim 2 \%$.

Electrons (muons) are subject to uncertainties on their energy (momentum) scale calibration and resolution, as well as their respective reconstruction and identification efficiencies. These uncertainties are derived from data using large samples of $J / \psi \rightarrow$ $\ell^{+} \ell^{-}$and $Z \rightarrow \ell^{+} \ell^{-}$decays $[139,140,141]$. The uncertainties on the predicted yields due to identification efficiency effects are $0.5-1.0 \%$ for muons, and $1.0-1.3 \%$ for electrons. The uncertainties on the expected yields due to muon and electron isolation efficiencies are typically $\sim 2 \%$ in size. Overall, in the $4 \ell$ channel, the lepton scale and resolution uncertainties are small with respect to the statistical uncertainties on the data yields, and the theoretical uncertainties presented in the following section. 
In terms of jet reconstruction, the uncertainties on the event yields in each reconstruction category due to jet energy scale and resolution effects are generally $<10 \%[142,143]$, although larger uncertainties of $\mathcal{O}(20 \%)$ are possible for low-yield regions, such as the ttH-enriched category. The uncertainties associated with the efficiency of the tagging algorithm used to identify $b$-quark jets, as derived from $t \bar{t}$ events, are at the percent level over most of the jet $p_{\mathrm{T}}$ range [116]. The latter uncertainty is only relevant in the ttH-enriched category, where $b$-quark jets are used directly, with the associated uncertainty being approximately 5\%. Uncertainties are also computed for jet flavour composition and response, which cover differences in jet calibration and correction factors which exist between quark- and gluon-initiated jets. These uncertainties are derived for each Higgs signal type, as well as the irreducible $Z Z^{*}$ background. The contribution of these uncertainties is at most $5-6 \%$ for a given signal or background process.

The impact of the precision of the Higgs boson mass $\left(m_{H}=125.09 \pm 0.24\right.$ $\mathrm{GeV}[144])$ on the signal acceptance was investigated as a potential source of uncertainty, due to the use of a mass window requirement to define the signal region. However, the effects of this choice were ultimately found to be negligible. A small dependency of the 0 -jet discriminant shape on $m_{H}$ is observed for the signal (below $2 \%$ in the highest BDT bins), which primarily affects measurements of ggF production, as well as the measurements in other categories with large concentrations of ggF events. Lastly, the uncertainties from the data-driven measurement of reducible background contributions tend to be less than $3 \%$. 


\subsection{Theoretical uncertainties}

The sources of theoretical uncertainty in this analysis may affect both signal and background yields in each experimental category, as well as the shapes of the discriminants used in the 0,1 , and $\geq 2$-jet categories. Perhaps the most critical aspect to consider is the effect of QCD scale variations on the ggF signal yields, and the resultant migrations of events in and out of $N$-jet bins, first introduced in Section 7.4. The choice of underlying PDF may also have non-trivial effects on the per-category yields, and applies to all signal and background processes. Lastly, the choice of SMC generator can modify the UE and MPI components of the simulation, as well as the evolution of the parton shower, which may affect both the event yields in each category, as well as the discriminant shapes. Therefore, each component of the simulation must be varied and evaluated carefully as a source of systematic uncertainty.

\subsubsection{QCD scale variation, bin migration uncertainties}

As previously described in Section 7.4, the uncertainty on the cross section of a given process due to missing higher-order terms in perturbative QCD is typically evaluated by varying the renormalization $\left(\mu_{\mathrm{R}}\right)$ and factorization $\left(\mu_{\mathrm{F}}\right)$ scales of the calculation. The scales are often varied at the same rate using a single scale variation parameter $\mu=\mu_{\mathrm{F}}=\mu_{\mathrm{R}}$, where $\mu$ can take on values of $\mu=\mu_{0} / 2$ or $2 \times \mu_{0}$ in comparison to the nominal choice, $\mu_{0}$. Therefore, the difference in yield between the nominal and upward/downward scale variations provides the uncertainty on the cross section $\Delta \sigma$, with the relative uncertainty $\Delta \sigma / \sigma$ propagated through the analysis.

However, when performing analyses based on exclusive jet bins, one must also consider the effect that such a separation induces on the cross section predictions 
for ggF production, particularly when fixed-order predictions are used. That is to say, the scale variation uncertainties on predictions may become unrealistic if cuts are made which separate inclusive $\geq N$-jet cross sections into exclusive $N$-jet and $\geq N+1$-jet components, as shown by Tackmann et al. $[95,134]$. While Section 7.4 detailed this behaviour for $H+2 j$ calculations at NLO QCD, in practice the same behaviour is visible for exclusive 0 - and 1-jet bins, as well.

Given that the current analysis methodology relies on cross section predictions made in exclusive jet bins, the total perturbative uncertainty for ggF must factor in both the cross sectional difference due to scale variations, as well as the effect of the jet binning on the perturbative uncertainty. During Run-I of the LHC, most ATLAS analyses which employed jet bins used an expanded version of the S-T method [134] for this purpose. Analogous to the case of a single cross section divided into two exclusive jet bins, the essential result of the expanded S-T method is that by dividing the inclusive ggF cross section into exclusive 0,1 , and $\geq 2$-jet bins, the perturbative uncertainties on each component can be evaluated as,

$$
\begin{gathered}
\Delta \sigma_{0}^{2}=\left(\Delta \sigma_{\geq 0}^{\mu}\right)^{2}+\left(\Delta \sigma_{\geq 1}^{\mu}\right)^{2}, \quad \Delta \sigma_{1}^{2}=\left(\Delta \sigma_{\geq 1}^{\mu}\right)^{2}+\left(\Delta \sigma_{\geq 2}^{\mu}\right)^{2}, \\
\Delta \sigma_{\geq 2}^{2}=\left(\Delta \sigma_{\geq 2}^{\mu}\right)^{2}
\end{gathered}
$$

where $\Delta \sigma_{\geq N}^{\mu}$ represents the absolute, inclusive scale variation uncertainty for the $\geq N$-jet ggF cross section, and $\Delta \sigma_{N}$ represents the total perturbative uncertainty for the exclusive $N$-jet cross section.

For purely fixed-order calculations which are sensitive to the presence of Sudakov 
logarithms, the above formulation provides a perfectly adequate estimate of the cross sectional uncertainty. However, in recent years, logarithmic resummation techniques have been developed for NNLO $\geq 0$-jet and NLO $\geq 1$-jet calculations [145], allowing for improved cross section predictions in perturbative QCD, as well as smaller associated uncertainties. Resummation is essentially a way to "reorganize" the terms in the perturbative expansion such that the divergent components become suppressed, and predictive power is restored to the calculation. For example, given a perturbative expansion for some observable $\hat{O}$ which has large Sudakov logarithms $L$,

$$
\hat{O}=1+\alpha_{s}\left(L^{2}+L+1\right)+\alpha_{s}^{2}\left(L^{4}+L^{3}+L^{2}+L+1\right)+\ldots
$$

the resummed expression may be rewritten as [146],

$$
\begin{aligned}
\hat{O} \rightarrow \exp \left(L g_{1}\left(\alpha_{s} L\right)+g_{2}\left(\alpha_{s} L\right)+\alpha_{s} g_{3}\left(\alpha_{s} L\right)+\ldots\right) C\left(\alpha_{s}\right) & \\
& +(\text { suppressed terms }),
\end{aligned}
$$

where including only the $g_{1}$ term would constitute a leading-logarithm (LL) resummation, while an expansion up to $g_{2}$ would be considered next-to-leading-logarithm (NLL). Worth noting is that resummation is not possible for all QCD processes, and even for processes which can be resummed, the calculations may be valid only at certain energy scales.

The Boughezal-Liu-Petriello-Tackmann-Walsh (BLPTW) method combines resummed $0 j$ and $1 j$ calculations with fixed-order $\geq 2 j$ calculations, providing an inclusive cross section estimate accurate to NNLO + NNLL, which is detailed in Ref. [145]. Aside from the improved cross section predictions, another important re- 
sult of the BLPTW approach is that theoretical covariances between the 0,1 , and $\geq 2$-jet cross sections can be derived, which are the result of both cross sectional uncertainties due to scale variations and jet bin migration effects (as in the original S-T method), as well as uncertainties from the choice of energy scale used in the resummation. In this construction, there are four sources of uncertainty to propagate - namely, the uncertainty from the choice of renormalization and factorization scales $\left(\Delta_{\mu}\right)$, the choice of resummation scale $\left(\Delta_{\phi}\right)$, and the migrations between the 0 and 1 -jet bins $\left(\Delta_{\text {cut }}^{0 / 1}\right)$, and the 1 and $\geq 2$-jet bins $\left(\Delta_{\text {cut }}^{1 / 2}\right)$.

Since the central values of the best-known ggF cross section predictions for 0,1 , and $\geq 2$-jet final states agree with the BLPTW predictions, and these predictions are used to compute the expected ggF event yields in the $H \rightarrow Z Z^{*} \rightarrow 4 \ell$ analysis, it stands to reason that the scale variation and migration uncertainties from the BLPTW approach can be propagated through the nominal ggF Monte Carlo sample in the $Z Z^{*}$ analysis, with their effects on the per-category yields used to estimate the components of the perturbative QCD uncertainty. To propagate these values, each uncertainty source serves as a "smearing factor" on the per-event Monte Carlo event weights. In particular, for the $i^{\text {th }}$ uncertainty source, the nominal weight for a given event is smeared as,

$$
w_{\text {Event }}^{\prime}=w_{\text {Event }} \times\left(1 \pm \frac{\Delta \sigma_{i}\left(N_{j}^{\text {true }}\right)}{\sigma_{i}\left(N_{j}^{\text {true }}\right)}\right)
$$

Here, the quantity $N_{j}^{\text {true }}$ is the number of truth-level jets in a given Monte Carlo event, and can assume values of 0,1 , or $\geq 2$. The corresponding values of $\sigma_{i}$ and $\Delta \sigma_{i}$ for each truth jet bin are tabulated in Ref. [31]. Worth noting is that the $0 / 1$ and $1 / 2$-jet bin migration uncertainties are treated as fully anti-correlated, since the 
covariance structure dictates that events can migrate back and forth between $N$ - and $(N+1)$-jet bins, but not simultaneously into both. All other uncertainty sources are treated as fully correlated.

Much in the same way that dividing ggF events into exclusive jet bins induces scale variation-based migration effects on the cross section predictions, further subdividing events into specific Higgs boson or jet $p_{\mathrm{T}}$ regions may also induce migration effects, enhancing the presence of unresummed Sudakov logarithms in the perturbative expansion. Therefore, it is necessary to consider additional uncertainties on the yields of the 1 -jet and $\geq 2$-jet bins which account for the migration of simulated events between low and high $p_{\mathrm{T}}$ regions. These migration effects were estimated using a shape uncertainty derived from the internal scale variations in a sample of PowHEG ggF events generated via the NNLOPS process. The uncertainties on the low and high $p_{\mathrm{T}}$ regions vary in size from $12-16 \%$ depending on the jet bin, and are anti-correlated, such that the uncertainty cancels when the $p_{\mathrm{T}}$ bins are joined together.

As described in Section 5.1, two approaches exist for calculating ggF cross sections: a more computationally intensive calculation which leaves the top quark mass finite; and a "Higgs effective field theory" approach which reduces the ggF production vertex to an effective contact interaction. Notable differences can emerge in the shape of the Higgs $p_{\mathrm{T}}$ spectrum when $p_{\mathrm{T}}^{H}>2 m_{t}$, therefore an additional source of uncertainty was considered to account for the differences caused by inclusion or exclusion of finite quark mass effects. In particular, a shape-based uncertainty was derived from samples of ggF events produced using Powheg NnLOPs with and without finite quark mass effects, similar to the $p_{\mathrm{T}}$ bin migration uncertainties. This uncertainty is negligible up to roughly $p_{\mathrm{T}}^{H}=150 \mathrm{GeV}$, but rises to $\mathcal{O}(40 \%)$ for $p_{\mathrm{T}}^{H} \sim 500 \mathrm{GeV}$. 
The resultant uncertainties due to scale variations / bin migrations and quark mass effects are shown in Figure 8.1, represented in terms of relative shifts $\Delta N_{i} / N_{i}$ on the nominal yields $N_{i}$ in each bin, and scaled such that the shifts are centred about one (i.e. zero uncertainty). To keep track of the correlations between uncertainty sources, it is necessary to propagate each parameter separately through the statistical analysis, rather than using a total uncertainty. This effect is important when considering migrations between adjacent jet bins, which are fully anti-correlated in the BLPTW construction. For example, in Figure 8.1, the upward variation for the 1 and $\geq 2$-jet migration uncertainty is below $\Delta N_{i} / N_{i}=1$ for the 1-jet categories, and above one for the $\geq 2$-jet categories, and vice versa for the downward variation. A similar trend is seen for the migration uncertainies between the 0- and 1-jet bins.

Because multivariate discriminants are used to differentiate between Higgs signal modes in each jet bin, one must also consider the effects that these variations have on the nominal BDT response. The scale variation / migration and quark mass effects are based on the true number of final state jets and Higgs boson $p_{\mathrm{T}}$ in each event, rather than the reconstructed quantities. Therefore, differences in reconstruction efficiency, pile-up effects, etc. can induce residual effects on the shapes of the BDT discriminants. Similar to the yield uncertainties of Figure 8.1, the effects on each discriminant are evaluated by smearing the event weights in the nominal ggF sample by the factors computed using Equation 8.4. Each discriminant distribution is then normalized to create a probability density function, with the bin-by-bin deviation in shape with respect to the nominal BDT response providing the uncertainty. The per-bin variations of the $\geq 2$-jet category discriminants are shown in Figure 8.2 for each theoretical uncertainty source considered. The BDT shape uncertainties 

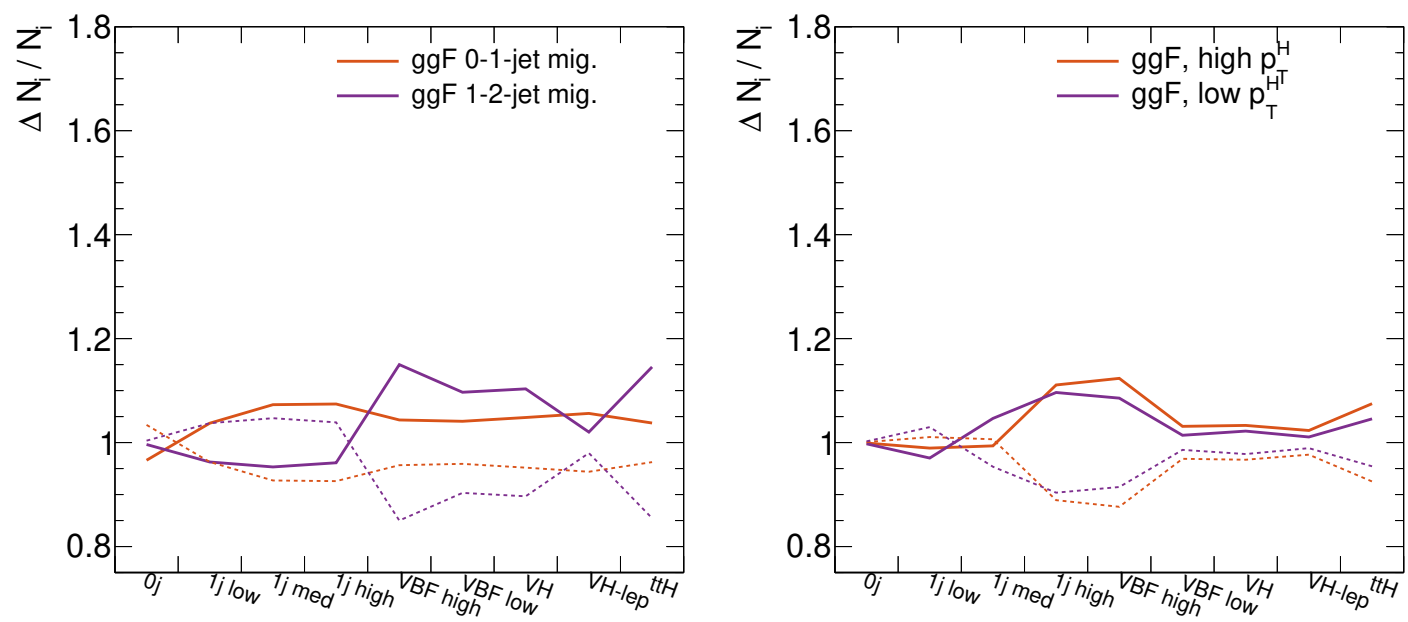

(a) Jet bin migration

(b) $p_{\mathrm{T}}^{H}$ bin migration
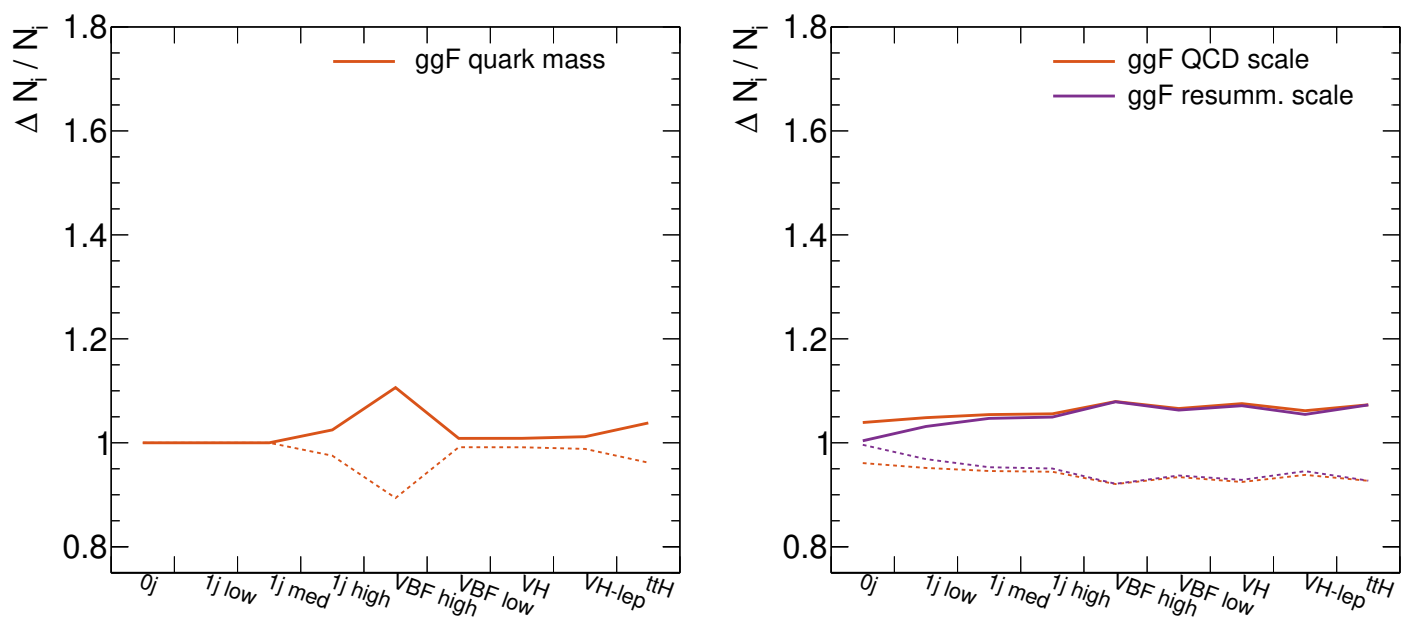

(c) Quark mass effects

(d) QCD, resummation scales

Figure 8.1: Theoretical uncertainties on the ggF cross section in each $4 \ell$ analysis bin, computed at $\sqrt{s}=13 \mathrm{TeV}$, due to QCD scale variations, bin migrations, and quark mass effects. 


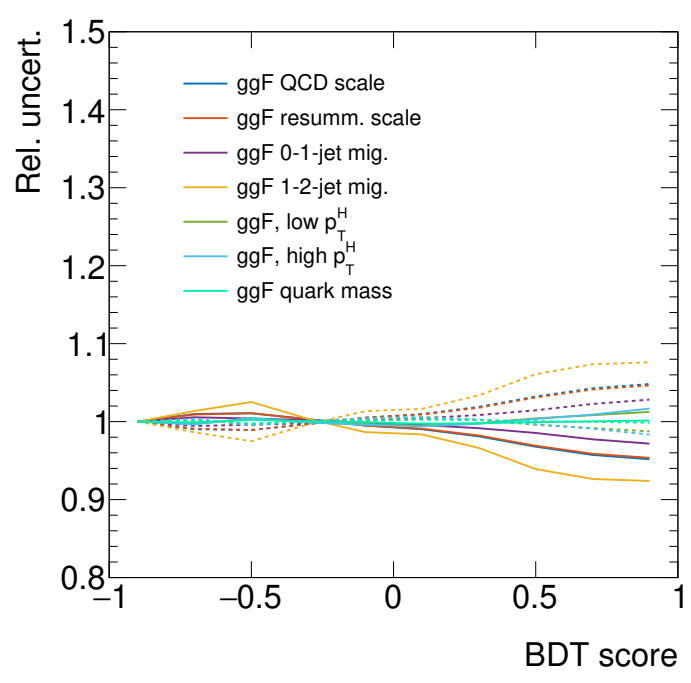

(a) VBF discriminant

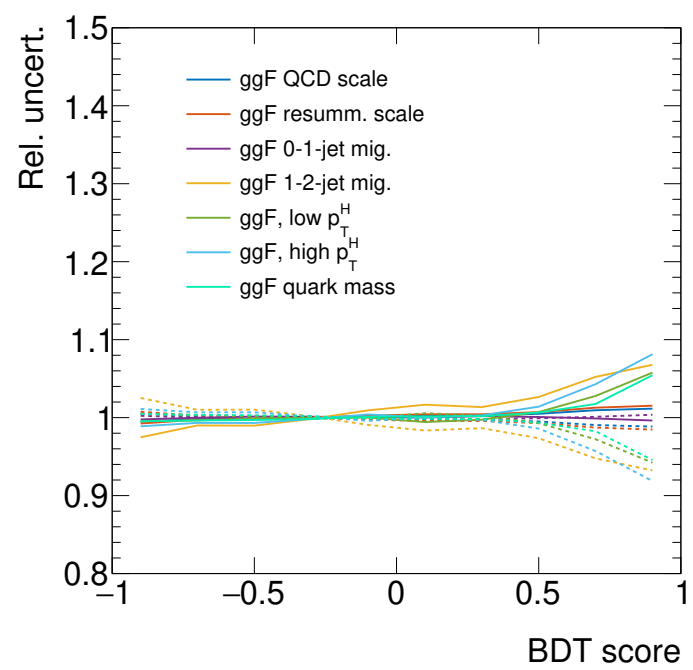

(b) $\mathrm{VH}$ discriminant

Figure 8.2: Theoretical uncertainties on the shapes of the BDT discriminants for ggF $H+2 j$ events due to QCD scale variations, bin migrations, and quark mass effects.

can be $10-15 \%$ in the least populated bins for gluon fusion production, but are generally negligibly small otherwise. For the $0 j$ and $1 j$ bin discriminants, the changes in discriminant shape are $<5 \%$ in any given bin, so these results are not shown.

Regarding VBF and VH production modes, these processes are entirely mediated by weak boson couplings at tree-level, so there is no danger of introducing Sudakov logarithms in the perturbative expansion when creating exclusive jet bins. Therefore, QCD scale variation uncertainties for these production modes can be derived through the usual technique of varying the renormalization/factorization scales of the process with respect to the nominal scale choices. Using such an approach yields relative uncertainties of $5-10 \%$ in each analysis category for both production modes, with mostly negligible changes in BDT discriminant shape. The QCD scale variation uncertainties for the irreducible $Z Z^{*}$ background are computed in the same manner as for VBF and VH Higgs production, with the per-category yield and discriminant 
uncertainties again computed from the envelope of $\mu_{\mathrm{F}}$ and $\mu_{\mathrm{R}}$ variations. The $Z Z^{*}$ background uncertainties are found to increase as a function of jet multiplicity, with the QCD uncertainty being $10-15 \%$ for events in the 0 - and 1-jet bins, and $30-40 \%$ for events with $\geq 2$ jets. The shape uncertainties for the per-category discriminants are of the same size as ggF Higgs production, with the uncertainties in less populated bins being $\mathcal{O}(10 \%)$, but negligible otherwise.

\subsubsection{PDF uncertainties}

Recalling that protons are composed of three partons (two up quarks, one down quark), along with sea quarks and gluons, the choice of PDF used to model the parton momentum distributions can have a non-trivial effect on both the cross section of the process, along with the kinematics of the final state particles. Generally, the uncertainties on the choice of PDF are calculated using internal variations (or eigenvectors) of a given $\mathrm{PDF}$ set, with the eigenvector variations propagated as smearing factors on the nominal event weights produced by the MC generator.

The absolute uncertainty in the $k^{\text {th }}$ category due to the $i^{\text {th }} \mathrm{PDF}$ eigenvector variation is computed as the difference in per-category event yield after applying the variant event weights for the $i^{\text {th }}$ eigenvector to the nominal sample. The relative uncertainty is then computed in the usual manner as $\Delta N_{i} / N_{i}$ and propagated through the analysis. For the Higgs boson signal Monte Carlo samples used in this analysis, PDF4LHC was chosen as the nominal PDF set, containing 30 internal variations, plus one upward/downward variation on $\alpha_{s}$. Rather than propagating the total uncertainty, it was decided that the individual eigenvector components would be propagated. This approach was taken to facilitate a later combination of measurements from Higgs 
analysis groups studying channels such as $H \rightarrow b \bar{b}$ and $H \rightarrow \tau \tau$, which are more sensitive to PDF-related effects, owing to the presence of QCD-mediated interactions in both Higgs production and decay.

The resultant per-category relative uncertainties are shown in Figure 8.3 for ggF production. To enhance readability, only eigenvectors which contribute a $0.5 \%$ uncertainty in at least one analysis category are shown. It is clear that the largest effect on the nominal yields comes from the $\alpha_{s}$ variation, which induces a relatively constant $2-3 \%$ change in yield per category. Other eigenvector variations tend to contribute $<1 \%$ uncertainty per category, such that the total PDF uncertainty from all variations is $\mathcal{O}(5 \%)$. The PDF variations for $\mathrm{VBF}$ and $\mathrm{VH}$ Higgs production contribute $\mathrm{a} \sim 2 \%$ uncertainty overall between each analysis category.

Similar to the scale variation uncertainties, the PDF eigenvector and $\alpha_{s}$ variations were also propagated to the per-category BDT discriminant shapes, and were found to have $a<2 \%$ effect per bin in each category. Lastly, the uncertainty on the irreducible $Z Z^{*}$ background is computed using an envelope of the maximum variations of the NNPDFnnlo 3.0 eigenvector set, and the difference of the nominal PDF choice with respect to two alternate PDF choices, CT14nnlo [147] and MMHT2014nnlo68cl [148]. The induced uncertainties were uniformly within the $1-2 \%$ range across all percategory yields and discriminants. Therefore, the effects of PDF variations in the $4 \ell$ decay channel are overall quite small in comparison to those induced by scale variations and jet $/ p_{\mathrm{T}}$ bin migrations. 


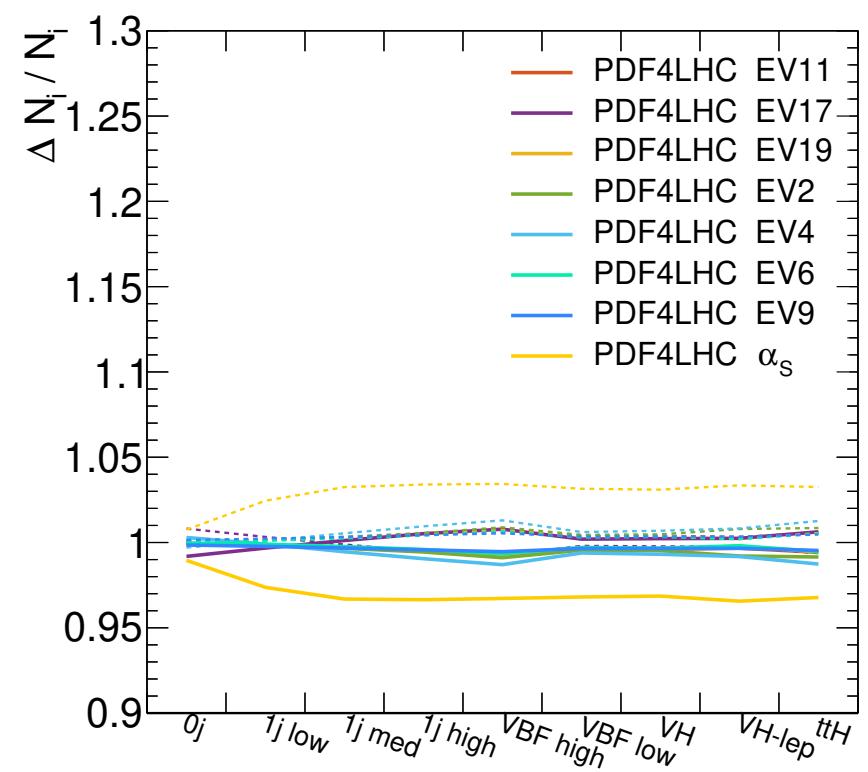

Figure 8.3: Theoretical uncertainties due to $\mathrm{PDF}$ eigenvector and $\alpha_{s}$ variations on the ggF cross section in each $4 \ell$ analysis bin, computed at $\sqrt{s}=13 \mathrm{TeV}$, using PDF4LHC as the nominal PDF choice.

\subsubsection{Parton showering, underlying event}

As previously mentioned, Shower Monte Carlo generators excel at modelling the behaviour of low-momentum particles found in quark and gluon hadronization, as well as multi-parton interactions. Because such processes are difficult to treat using perturbative QCD, a SMC generator will require the use of tunes to model the nonperturbative components of these processes. These tunes are often data-driven, employing hadronic data from LEP or the LHC to improve the quality of the simulation. In this regard, the choice of tune will affect both the description of the underlying event, as well as the evolution of each parton shower.

Several possible sources of variation exist in the parton showering component of the $\mathrm{MC}$ event generation: variations in the nominal SMC generator tune, e.g. the choice of multi-parton interaction model or FSR model; the choice of SMC generator tune; 
and the choice of SMC generator (such as Pythia8 vs. HeRWiG). In this analysis, for all but the last item, the parton showering is done via PYTHIA8, with uncertainties coming from variations in the generator configuration. The individual effects on the per-category event yields can either be summed in quadrature to produce a total uncertainty, or propagated as separate uncertainties through the analysis.

The nominal choice of SMC generator tune for this analysis is AZNLO, with variations on this nominal tune coming in the form of changes to the renormalization and factorization scale choices, initial momenta of the partons, variations on the configuration of the MPI model, and the choice of $p_{\mathrm{T}}$ cutoffs used to treat the divergences in $\mathrm{QCD}$ as $p_{\mathrm{T}} \rightarrow 0$ for the initial partons. As with the PDF and scale uncertainties, the relative uncertainty in the $k^{\text {th }}$ category is computed using the variation in event yield, with the main difference being that variant yield is not a product of smearing the nominal event weights, but rather samples of the same parton-level events which have been showered using variant configurations, then subject to the same reconstruction. The uncertainties on the per-category yields for each parameter variation are shown in Figure 8.4 for ggF events. It is evident that only the use of FSR has a meaningful impact on the final yields, being a $3-4 \%$ effect for events with two or more jets. For events with fewer final state jets, the effect of this variation is less pronounced.

To estimate the effect of different tunes in Pyтhia8 on the final per-category yields, the nominal parton-level events can also be showered using four ATLASstandard tunes - in particular, CTEQ6L1, MSTW2008LO, NNPDF23LO, and HERAPDF15LO - which together constitute the A14 (ATLAS 2014) tune series. Due to the fact that each tune has its own internal variations, the overall uncertainty is derived using a simple envelope of the upward and downward variations of the four constituent A14 


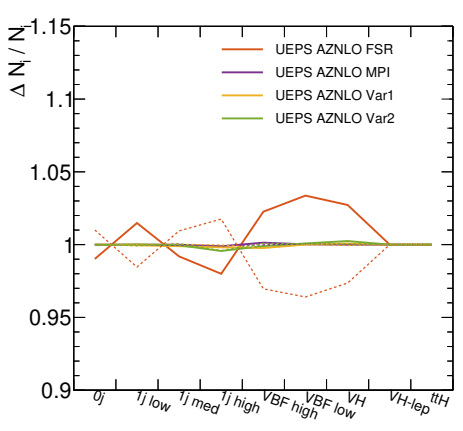

(a) AZNLO tune

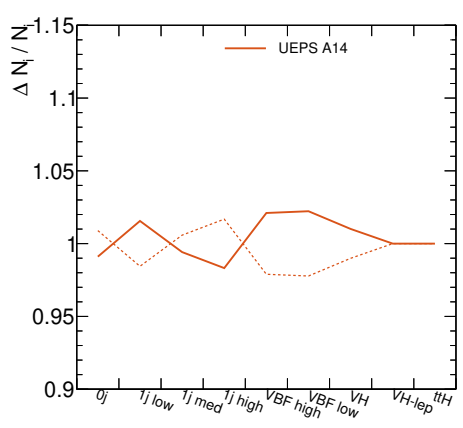

(b) A14 variation

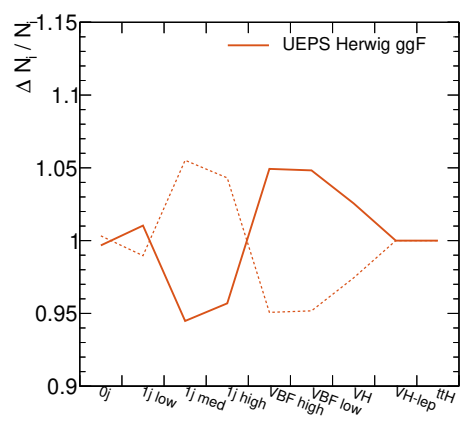

(c) Herwig variation

Figure 8.4: Uncertainties due to variations in the choice and configuration of the parton shower used to generate truth-level $\mathrm{ggF}$ events.

tunes, as compared to the nominally-showered sample. The envelope of the largest upward/downward variations in yield with respect to the nominal sample thus constitutes the A14 uncertainty. The resultant uncertainties are shown in Figure 8.4 for ggF events, with the choice of SMC tune having at most a $\sim 2 \%$ effect on the yield.

Lastly, in order to assess the effect of the choice of SMC generator on the percategory yields, the same parton-level events were showered separately using the Pythia8 and Herwig SMC generators. The events were showered using the AZNLO tune, with the uncertainty coming from the change in per-category yields induced by the choice of SMC generator. The effects of the choice of HERWIG are slightly more visible, with the relative uncertainty varying at most by $\sim 5 \%$ between categories, as shown in Figure 8.4. Regarding VBF + VH production, the uncertainties from each variation source are of comparable magnitude to those computed for ggF production. The uncertainties from each source tend to be $\leq 5 \%$, although they can be upwards of $15 \%$ in the 0 -jet region, where the event yields are generally the lowest for VBF and $\mathrm{VH}$ events. 
The SMC generator variations were also used to estimate uncertainties on the shapes of the per-category BDT discriminants. The discriminant shape uncertainties for ggF events are shown in Figures 8.5 for the HERWIG and A14 variations, and 8.6 for internal AZNLO variations. While the effect on the per-category yields is rather small, the impact on the shape of the discriminants can be quite non-trivial when varying the nominal tuning parameters, or choice of SMC generator, particularly in the extrema of the BDT score range. These large variations are not physically-motivated per se, but instead are a (rather unfortunate) function of the limited number of events in the variant samples used for the SMC uncertainty calculations. The first and last BDT bins tend to be less populated on average than the others, hence they are more susceptible to fluctuations in yield for small MC samples, which will in turn affect the normalized shape of the BDT discriminant. In future iterations of the analysis, increasing the size of the variant samples would provide a more robust (and likely smaller) estimate of each shape uncertainty. Similar trends in the discriminant shape uncertainties are observed for VBF and VH Higgs production, as well.

\subsection{4 $k$-factor variations with anomalous couplings}

As described in Section 6.7, a "best prediction" scaling is applied for the Higgs signal samples generated with anomalous couplings, which ensures that the signal predictions converge to their best-known SM values when all anomalous coupling terms vanish. This scaling approach amounts to the use of per-category $k$-factors applied to each signal sample, which are assumed to be constant over the entire coupling parameter space. However, there is some evidence of variation in the LO-to-NLO $k$-factor for $\mathrm{VBF}+\mathrm{VH}$ events with the introduction of anomalous couplings [97], ne- 


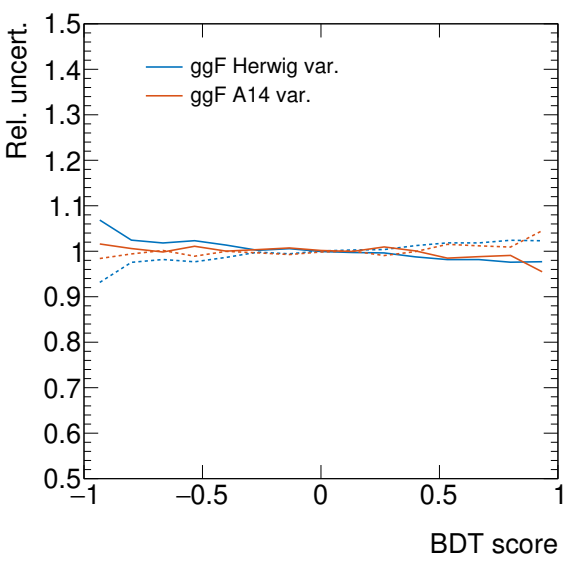

(a) 0 -jet

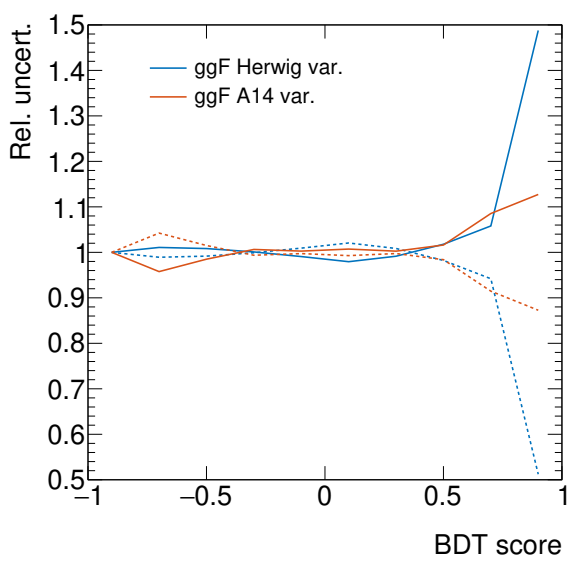

(c) 1 -jet, $60<p_{\mathrm{T}}^{H}<120 \mathrm{GeV}$

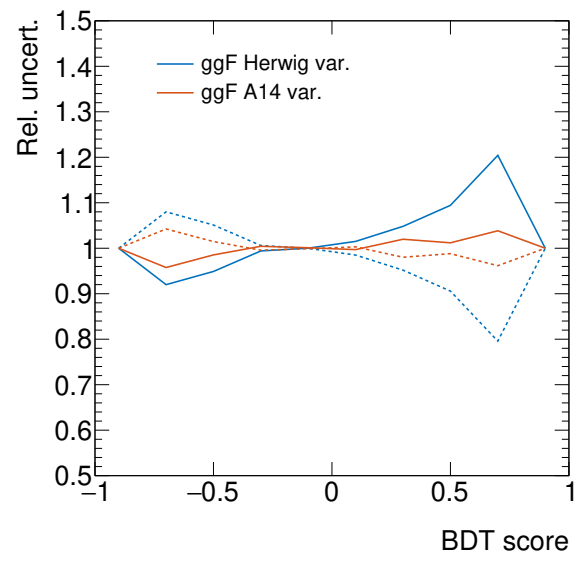

(b) 1 -jet, $p_{\mathrm{T}}^{H}<60 \mathrm{GeV}$

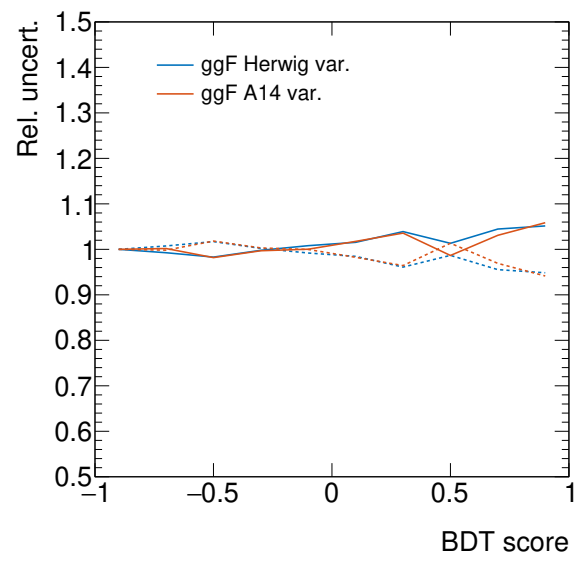

(d) 2-jet, VBF-enriched

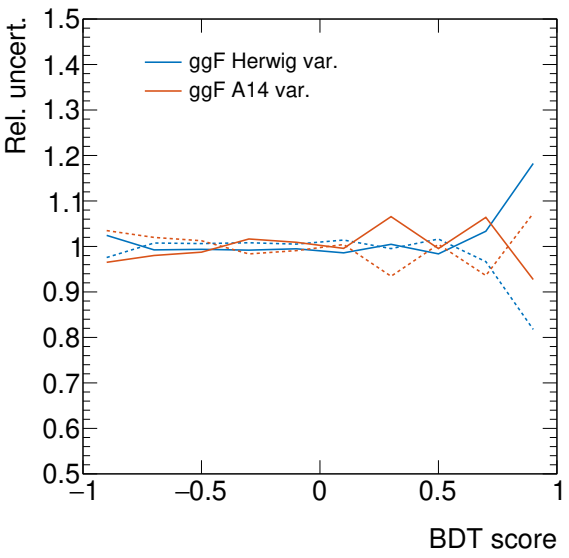

(e) 2-jet, VH-enriched

Figure 8.5: Shape uncertainties due to choice of SMC generator and tune for the BDT discriminants in $\mathrm{ggF} H \rightarrow Z Z^{*} \rightarrow 4 \ell$ events. 


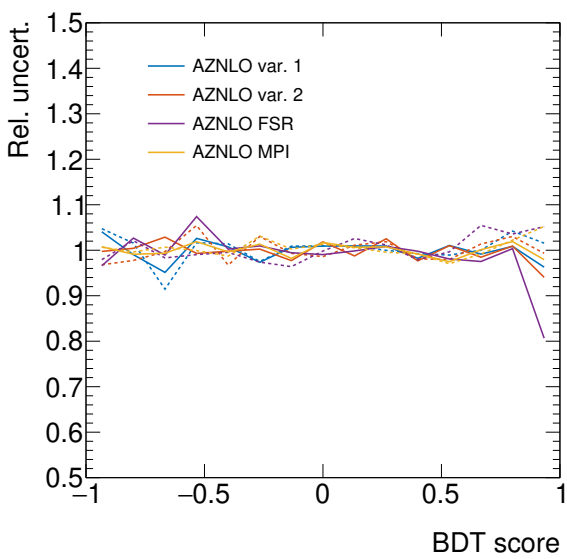

(a) 0 -jet

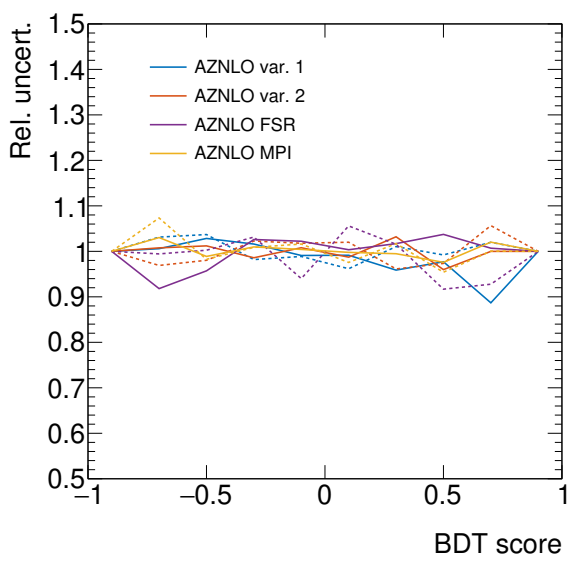

(c) 1 -jet, $60<p_{\mathrm{T}}^{H}<120 \mathrm{GeV}$

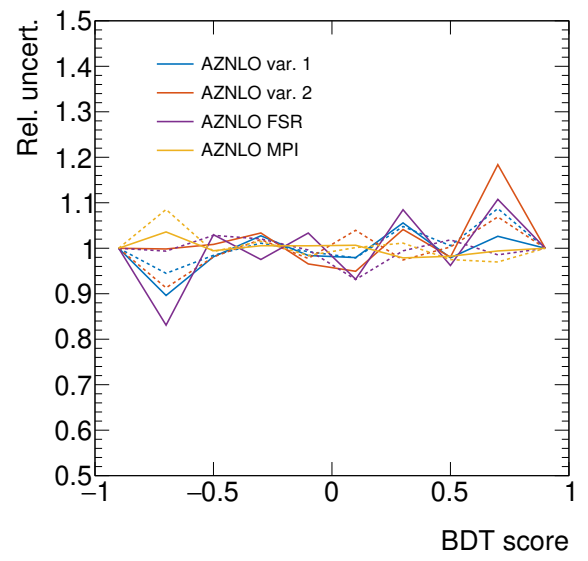

(b) 1 -jet, $p_{\mathrm{T}}^{H}<60 \mathrm{GeV}$

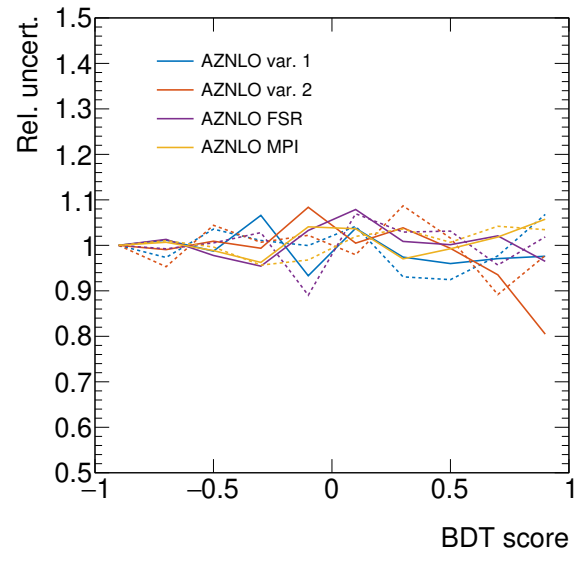

(d) 2-jet, VBF-enriched

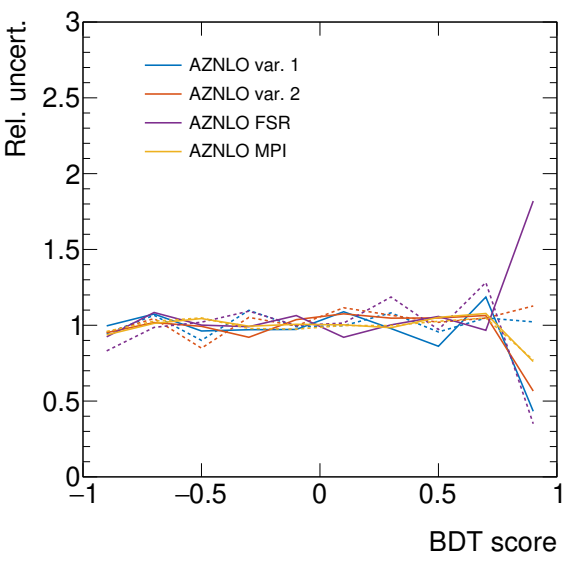

(e) 2-jet, VH-enriched

Figure 8.6: Shape uncertainties due to variations in the nominal PYTHIA8 AZNLO tune for the BDT discriminants in $\mathrm{ggF} H \rightarrow Z Z^{*} \rightarrow 4 \ell$ events. 
cessitating the evaluation of the uncertainty introduced by using $k$-factors exclusively based on the SM coupling point. To this end, the relative uncertainty on the $k$-factor in the $j^{\text {th }}$ category is computed as,

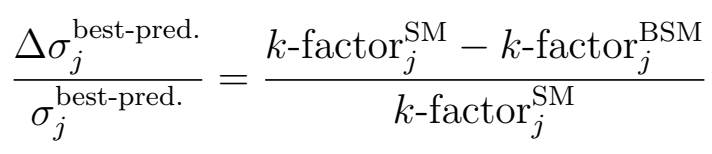

To compute the $k$-factor ${ }_{j}^{\mathrm{BSM}}$ terms, truth-level LO and NLO samples of $\mathrm{VBF}+\mathrm{VH}$ $H \rightarrow 4 \ell$ events are generated, with the histogram morphing technique described in Section 5.4 used to compute the LO-to-NLO variation at a given point in the coupling parameter space. As such, the uncertainty on the $k$-factor is dynamically computed for each category according to the BSM hypothesis being tested. The resultant uncertainties vary between $10 \%$ to $40 \%$, with larger uncertainties typically only observed in the ttH-enriched category, owing to the low number of MC events (and subsequent yield) in this region. Worth noting is that no additional uncertainties of this form are necessary for ggF-mediated Higgs signal, as these MC samples were computed at NLO QCD. 


\section{Chapter 9}

\section{Signal strength measurements,}

\section{limits on anomalous couplings, and}

\section{outlook}

This chapter explains the $H \rightarrow Z Z^{*} \rightarrow 4 \ell$ statistical analysis methodology, in which the optimized discriminant detailed in Chapter 7 was integrated as a means to improve the overall sensitivity to VBF-mediated $H \rightarrow 4 \ell$ signal. The expected and

observed event yields from $\int \mathcal{L}=36.1 \mathrm{fb}^{-1}$ of ATLAS data recorded at $\sqrt{s}=13 \mathrm{TeV}$ are shown, along with the results of the statistical analysis. Using the same data, constraints are also placed on the presence of anomalous couplings in the Higgs boson production and $4 \ell$ decay vertices. 


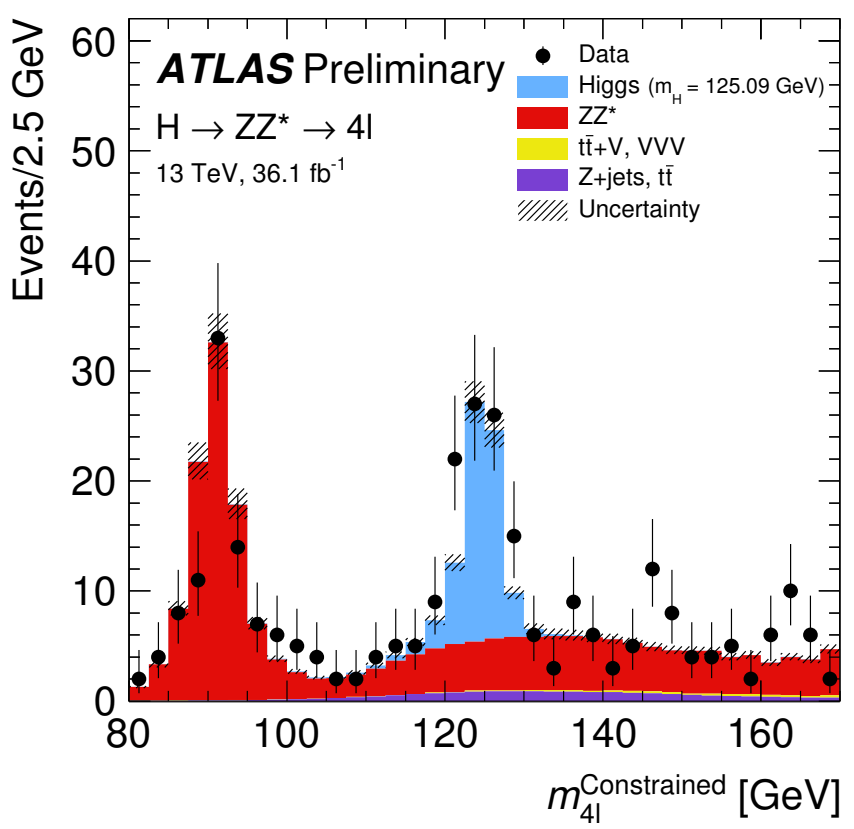

Figure 9.1: Inclusive distribution of the invariant mass $m_{4 \ell}$ in data, superimposed on MC normalized to the SM prediction for $36.1 \mathrm{fb}^{-1}$ at $\sqrt{s}=13 \mathrm{TeV}$.

\subsection{Event yields and discriminant shapes}

The observed yields in each reconstruction category, along with the corresponding SM expectations, are summarized in Table 9.1 for an invariant mass region $118<m_{4 \ell}<129 \mathrm{GeV}$. The total observed number of events is around $23 \%$ higher than the SM signal and background expectation, driven largely by excesses in the VBF-enriched categories. There are no events observed in the leptonic VH-enriched and ttH-enriched categories, which are both consistent with the SM prediction. Conversely, the observed yields in the VBF-enriched categories are roughly double and quadruple the expected yields for the low and high jet $p_{\mathrm{T}}$ categories, respectively. The inclusive distribution of the constrained $m_{4 \ell}$ is shown in Figure 9.1, while the observed discriminant distributions are shown superimposed with the MC expecta- 
tions in Figure 9.2. Furthermore, Figure 9.3 superimposes the expected and observed distributions of the training variables used to build the VBF discriminant in the kinematic region with $m_{\mathrm{jj}}>120 \mathrm{GeV}$ and $p_{\mathrm{T}}^{j_{1}}<200 \mathrm{GeV}$.

\begin{tabular}{lccccc}
\hline \hline $\begin{array}{l}\text { Reconstruction } \\
\text { category }\end{array}$ & Signal & $\begin{array}{c}Z Z \\
\text { background }\end{array}$ & $\begin{array}{c}\text { Other } \\
\text { backgrounds }\end{array}$ & $\begin{array}{c}\text { Total } \\
\text { expected }\end{array}$ & Observed \\
\hline 0-jet & $26.8 \pm 2.5$ & $13.7 \pm 1.0$ & $2.23 \pm 0.31$ & $42.7 \pm 2.7$ & 49 \\
1 -jet, $0<p_{\mathrm{T}}^{4 \ell}<60 \mathrm{GeV}$ & $8.8 \pm 1.1$ & $3.1 \pm 0.4$ & $0.53 \pm 0.07$ & $12.5 \pm 1.2$ & 12 \\
1 -jet, $60<p_{\mathrm{T}}^{4 \ell}<120 \mathrm{GeV}$ & $5.4 \pm 0.7$ & $0.88 \pm 0.12$ & $0.38 \pm 0.05$ & $6.7 \pm 0.7$ & 9 \\
1 -jet, $p_{\mathrm{T}}^{4 \ell}>120 \mathrm{GeV}$ & $1.47 \pm 0.24$ & $0.139 \pm 0.022$ & $0.045 \pm 0.007$ & $1.65 \pm 0.24$ & 3 \\
VBF, $p_{\mathrm{T}}^{j_{1}}<200 \mathrm{GeV}$ & $6.3 \pm 0.8$ & $1.08 \pm 0.32$ & $0.40 \pm 0.04$ & $7.7 \pm 0.9$ & 16 \\
VBF, $p_{\mathrm{T}}^{j_{1}}>200 \mathrm{GeV}$ & $0.57 \pm 0.10$ & $0.093 \pm 0.032$ & $0.054 \pm 0.006$ & $0.72 \pm 0.10$ & 3 \\
VH-hadronic, $p_{\mathrm{T}}^{4 \ell}<150 \mathrm{GeV}$ & $2.9 \pm 0.5$ & $0.63 \pm 0.16$ & $0.169 \pm 0.021$ & $3.7 \pm 0.5$ & 3 \\
VH-hadronic, $p_{\mathrm{T}}^{4 \ell}>150 \mathrm{GeV}$ & $0.64 \pm 0.09$ & $0.029 \pm 0.008$ & $0.0182 \pm 0.0022$ & $0.69 \pm 0.09$ & 0 \\
VH-leptonic & $0.318 \pm 0.019$ & $0.049 \pm 0.008$ & $0.0137 \pm 0.0019$ & $0.380 \pm 0.020$ & 0 \\
ttH & $0.39 \pm 0.04$ & $0.014 \pm 0.006$ & $0.07 \pm 0.04$ & $0.47 \pm 0.05$ & 0 \\
\hline Total & $54 \pm 4$ & $19.7 \pm 1.5$ & $3.9 \pm 0.5$ & $77 \pm 4$ & 95 \\
\hline \hline
\end{tabular}

Table 9.1: The observed and expected signal and background yields in each reconstructed event category, within the invariant mass range $118<m_{4 \ell}<129 \mathrm{GeV}$, for an integrated luminosity of $36.1 \mathrm{fb}^{-1}$ recorded at $\sqrt{s}=13 \mathrm{TeV}$. The expected yields assume the SM Higgs boson signal with a mass $m_{H}=125.09 \mathrm{GeV}$. Statistical and systematic uncertainties are added in quadrature.

\subsection{Higgs boson signal strength measurements}

To perform the statistical analysis in the $H \rightarrow Z Z^{*} \rightarrow 4 \ell$ channel, a likelihood function $\mathcal{L}(\vec{\mu}, \vec{\theta})$ is constructed as a product of conditional probabilities within bins of the per-category discriminant observables described in Chapter 7. In particular, the likelihood function is a product of Poisson-distributed probabilities $P$ corresponding to the observation of $N_{i, j}$ events in the $i^{\text {th }}$ bin of the $j^{\text {th }}$ category observable, given the expected signal and background yields $S_{i, j}(\vec{\mu}, \vec{\theta})$ and $B_{i, j}(\vec{\theta})$, respectively. This function is altogether expressed as, 


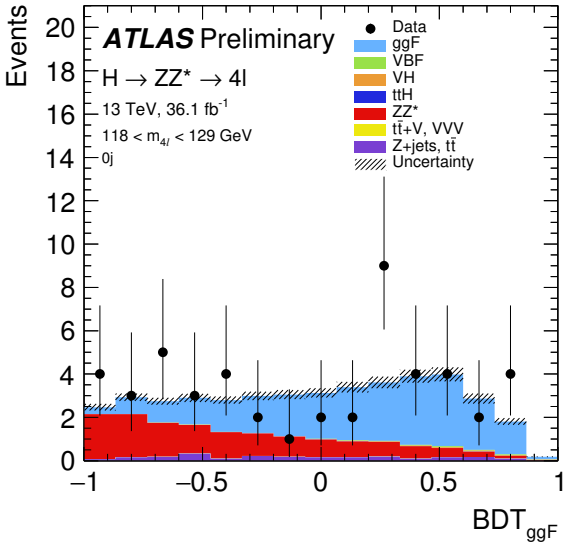

(a) 0 -jet

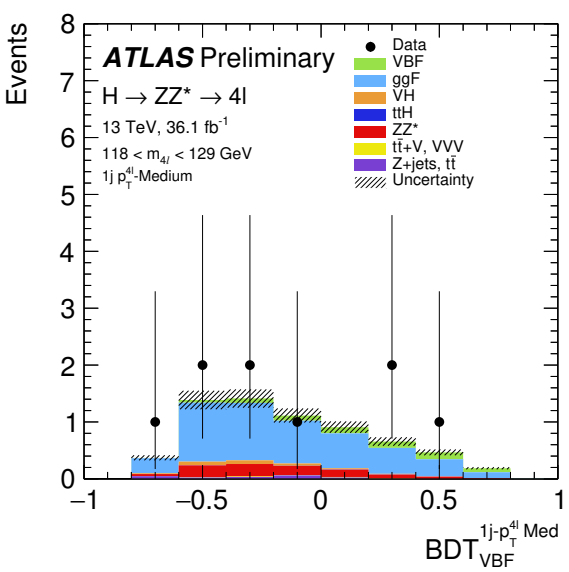

(c) 1 -jet, $60<p_{\mathrm{T}}^{H}<120 \mathrm{GeV}$

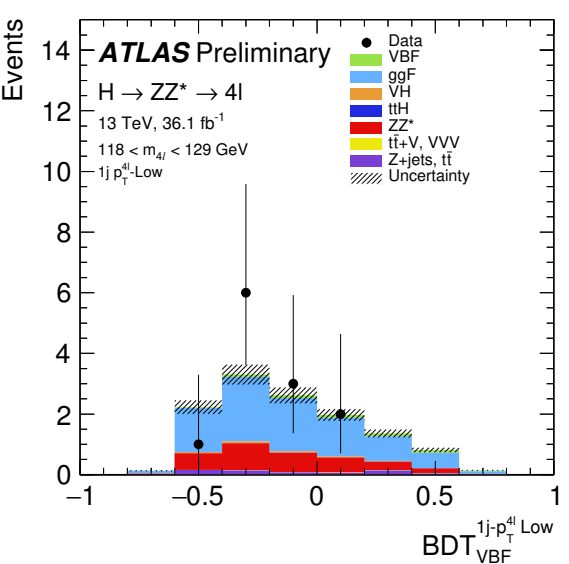

(b) 1 -jet, $p_{\mathrm{T}}^{H}<60 \mathrm{GeV}$

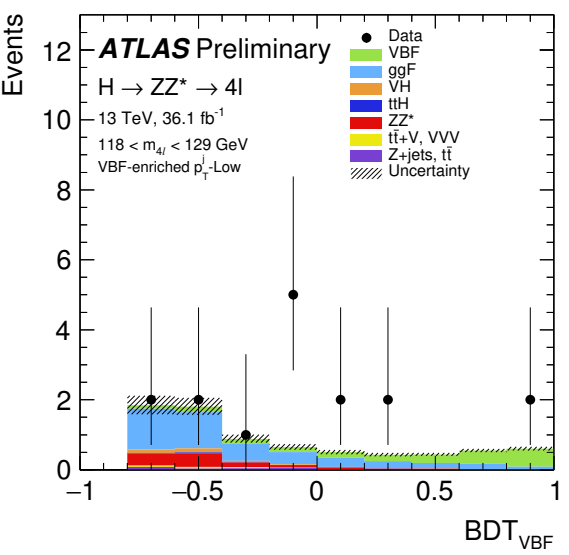

(d) $\geq 2$-jet, VBF-enriched

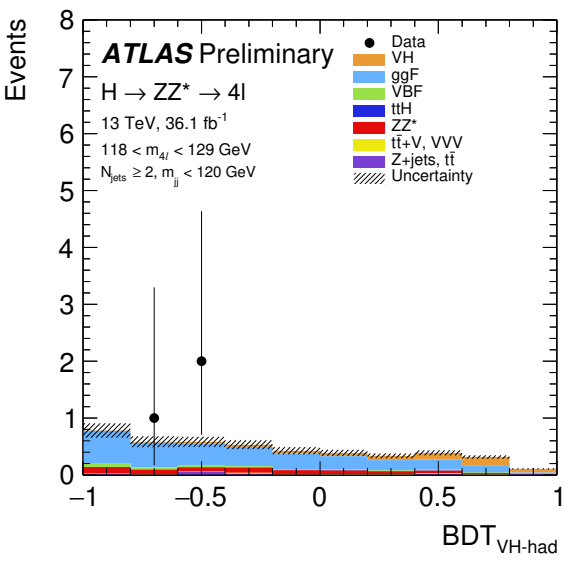

(e) $\geq 2$-jet, VH-hadronic-enriched

Figure 9.2: Distributions of the per-category discriminants in data, superimposed on MC normalized to the SM prediction for $\mathcal{L}=36.1 \mathrm{fb}^{-1}$ recorded at $\sqrt{s}=13 \mathrm{TeV}$. 


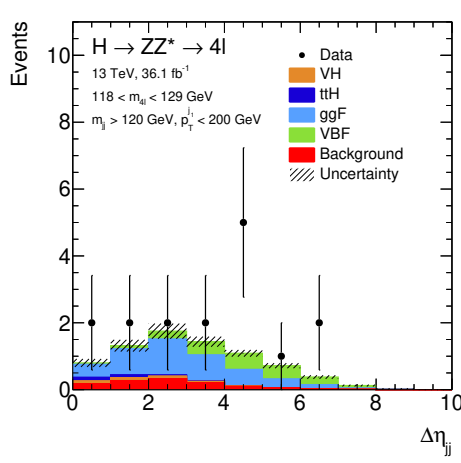

(a) $\Delta \eta_{\mathrm{jj}}$

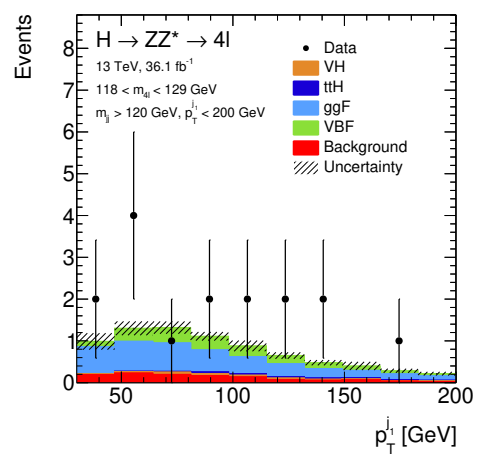

(d) $p_{\mathrm{T}}^{j_{1}}$

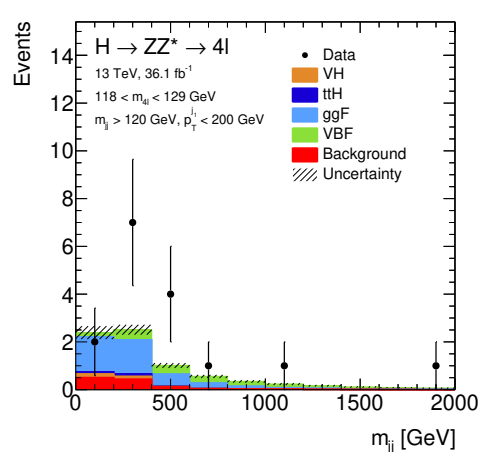

(b) $m_{\mathrm{jj}}$

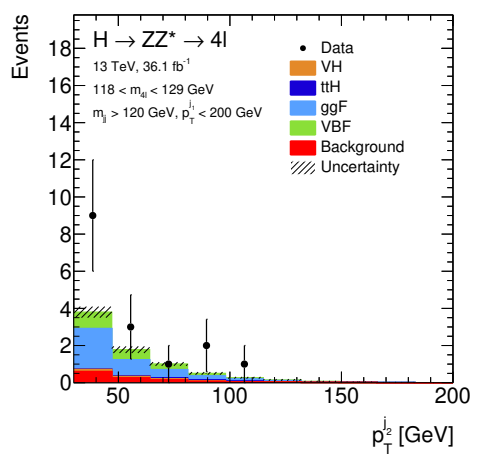

(e) $p_{\mathrm{T}}^{j_{2}}$

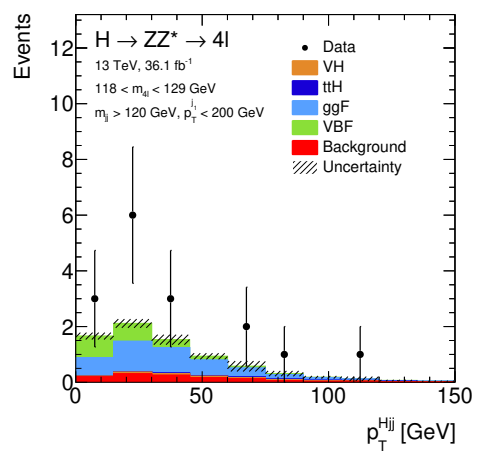

(g) $p_{\mathrm{T}}^{H j j}$

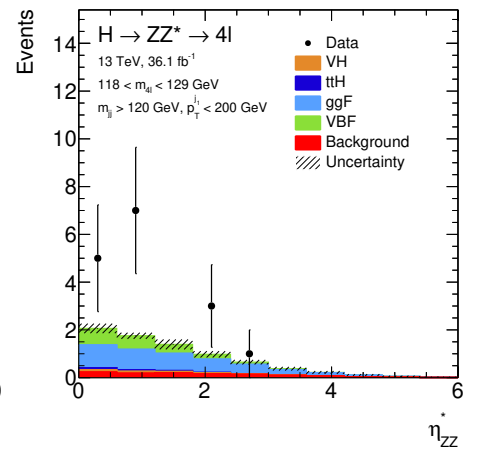

(c) $\eta_{Z Z}^{*}$

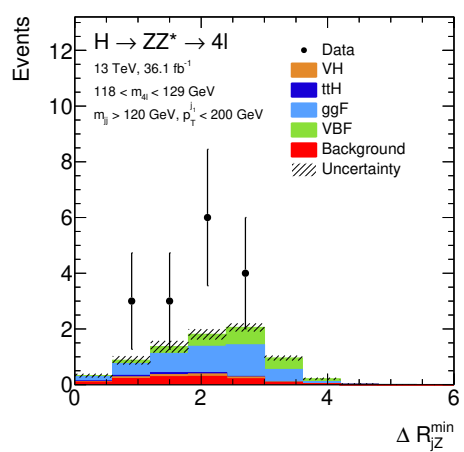

(f) $\Delta \mathrm{R}_{j Z}^{\min }$

Figure 9.3: Distributions of the kinematic variables used to train the VBF discriminant in both data and MC, shown in the kinematic region with $m_{\mathrm{jj}}>120 \mathrm{GeV}$ and $p_{\mathrm{T}}^{j_{1}}<200 \mathrm{GeV}$. 


$$
\mathcal{L}(\vec{\mu}, \vec{\theta})=\prod_{j}^{N_{\text {categories }}} \prod_{i}^{N_{\text {bins }}} P\left(N_{i, j} \mid S_{i, j}(\vec{\mu}, \vec{\theta})+B_{i, j}(\vec{\theta})\right) \times \prod_{m}^{N_{\text {nuisance }}} \mathcal{C}_{m}(\vec{\theta}) .
$$

In this expression, the symbol $\vec{\theta}=\left\{\theta_{1}, \theta_{2}, \ldots, \theta_{N}\right\}$ represents all nuisance parameters. Some of the nuisance parameters are constrained by auxiliary measurements characterizing detector acceptance, resolution, and reconstruction efficiency, with corresponding constraints represented by the functions $\mathcal{C}_{m}(\vec{\theta})$. The parameter of interest (POI) for this analysis is the vector of signal strengths $\vec{\mu}$, where $\vec{\mu}=\vec{\sigma} / \vec{\sigma}_{\mathrm{SM}}$. In this construction, the signal strengths for each Higgs production mode are treated as free parameters, with the signal strength for a given mode (e.g. $\left.\mu_{\mathrm{VBF}}\right)$ being the same across each experimental category. The test statistic for this analysis is based on a ratio of profiled likelihoods [131],

$$
q=-2 \log \frac{\mathcal{L}(\vec{\mu}, \hat{\vec{\theta}}(\vec{\sigma}))}{\mathcal{L}(\hat{\vec{\mu}}, \hat{\vec{\theta}})}
$$

where $\hat{\vec{\mu}}$ and $\hat{\vec{\theta}}$ are unconditional maximum likelihood estimators, and $\hat{\vec{\theta}}$ is the conditional maximum likelihood estimator under the specific $\vec{\mu}$ hypothesis.

The test statistic is used to compare the likelihoods of different signal strength hypotheses, allowing for a given hypothesis to be excluded (or not excluded) relative to the best-fit scenario. That is to say, large values of $q$ are interpreted as the data being in disagreement with the tested hypothesis in favour of the best-fit hypothesis. In practice, the analysis is done twice: once with the POI being a single number, used to obtain a measurement of the overall Higgs boson signal strength; and once with the 
POI being a four-dimensional vector, used to fit separately for each Higgs production mode of interest (i.e. ggF, VBF, VH, ttH). The likelihood scan performed is based on the assumption that the asymptotic approximation is valid, meaning that the test statistic follows a non-central $\chi^{2}$ distribution with one (four) degrees of freedom when fitting the overall (per-production mode) signal strength [131].

The inclusive, VBF, and per-production mode signal strength measurements are shown in Figure 9.4, with the limits in the inclusive and VBF scenarios shown with and without the inclusion of systematic uncertainties. The measured signal strengths and confidence limits are also summarized in Table 9.2. The use of BDT discriminants in the relevant reconstruction categories was found to improve the expected statistical uncertainties on the signal strength measurements by $15 \%, 25 \%$, and $35 \%$ for the ggF, $\mathrm{VH}$, and VBF production modes, respectively. While an excess in the inclusive signal strength is observed, the value of $\mu$ is still compatible with the SM prediction of $\mu=1$ within $1.3 \sigma$. It is clear from the inclusive $\mu$ that the limits in the $4 \ell$ channel are still quite driven by statistical uncertainty, as the inclusion of all systematic uncertainties increases the observed limits by $\sim 10 \%$ at most. With regard to the perproduction mode measurements, the observed excess focused in the VBF categories leads $\mu_{\mathrm{VBF}}$ to deviate from SM predictions by roughly $2 \sigma$, with the measured VBF signal strength being $\mu_{\mathrm{VBF}}=3.95_{-1.48}^{+1.73}$. For the $\mathrm{VH}$ and ttH production modes, only upper bounds can be set, as insufficient yields were recorded in data to constrain these signal strength parameters.

With regard to the deviation of $\mu_{\mathrm{VBF}}$ from SM predictions, it is worth discussing potential underlying explanations for the observed excess. Roughly half of the excess events are found to have BDT score $>0$, and thus are considered SM "VBF-like" 


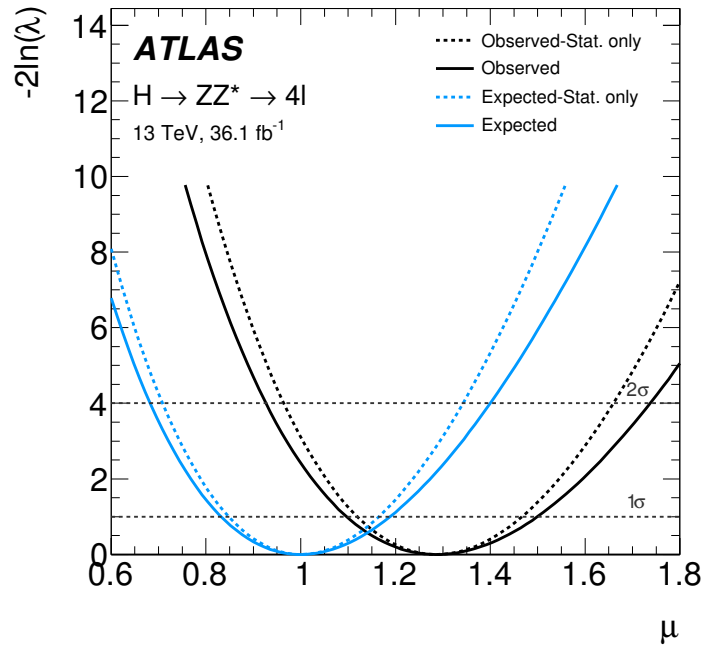

(a) Inclusive signal strength

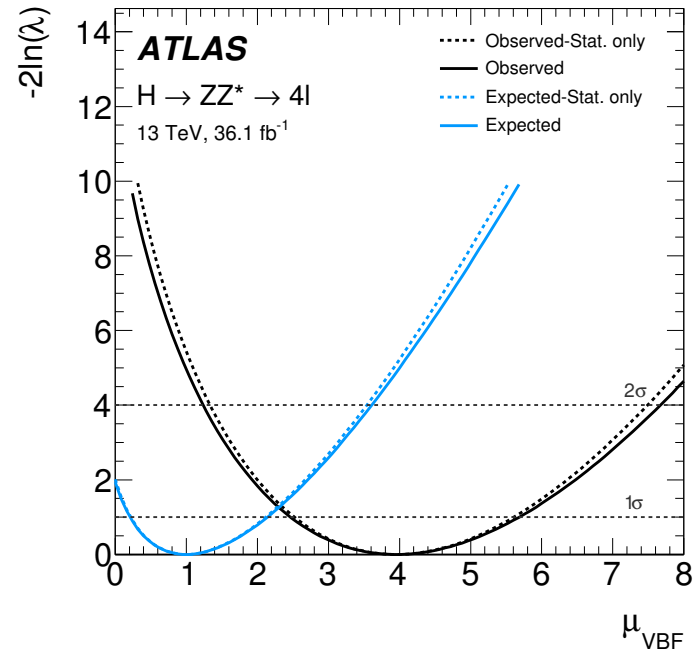

(b) VBF signal strength

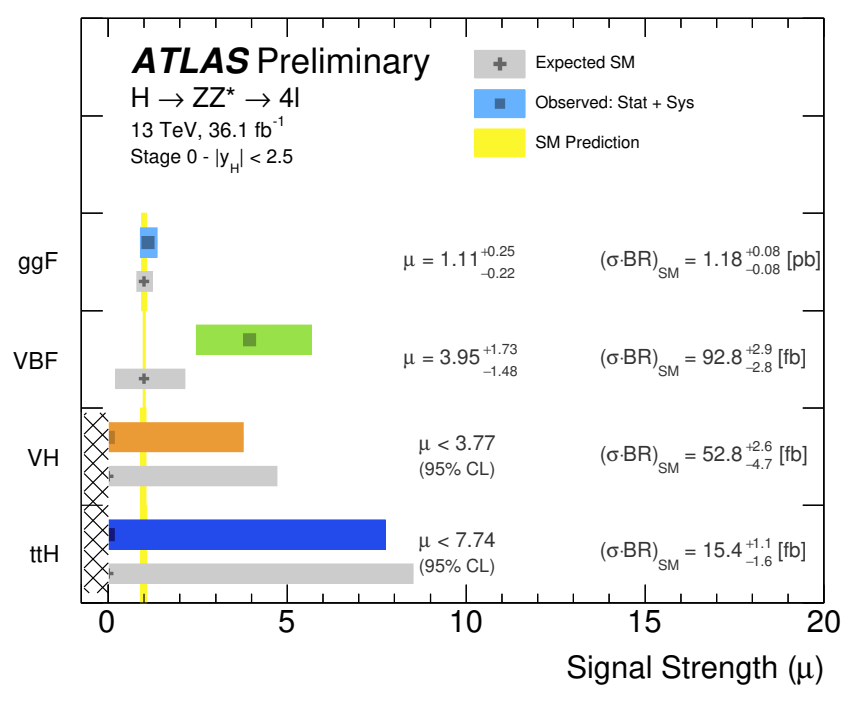

(c) Per-production mode signal strength

Figure 9.4: The observed inclusive signal strength measurement (top), as well as the signal strength per production mode (bottom), for $36.1 \mathrm{fb}^{-1}$ of data recorded at $\sqrt{s}=13 \mathrm{TeV}$. 


\begin{tabular}{lcc}
\hline \hline & \multicolumn{2}{c}{ Signal strength $(\mu)$} \\
Production mode & SM expected & Observed \\
\hline ggF & $1.00 \pm 0.07$ & $1.11_{-0.22}^{+0.22+0.07} \pm 0.04$ \\
$\mathrm{VBF}$ & $1.00 \pm 0.03$ & $3.95_{-1.40}^{+1.70} \pm 0.30 \pm 0.30$ \\
$\mathrm{VH}$ & $1.00_{-0.09}^{+0.06}$ & $<3.77$ \\
$\mathrm{ttH}$ & $1.00_{-0.10}^{+0.07}$ & $<7.74$ \\
\hline Inclusive & $1.00 \pm 0.07$ & $1.29_{-0.17}^{+0.18+0.07} \pm 0.03$ \\
\hline \hline
\end{tabular}

Table 9.2: Expected and observed signal strength values at $36.1 \mathrm{fb}^{-1}$ of $\sqrt{s}=13 \mathrm{TeV}$ data in the $H \rightarrow Z Z^{*} \rightarrow 4 \ell$ channel, made using the inclusive sample, and for each considered Higgs production mode. The relative uncertainties on each measurement are presented in components as $($ statistical $)+($ experimental $)+($ theoretical $)$.

events. So, it is perhaps not a surprising result that $\mu_{\mathrm{VBF}}$ grows to account for this excess, since VBF production is the dominant contribution in this region. With respect to the plots shown in Figure 9.3, one can see that the BDT score distribution is reflected in the kinematic distributions of the training variables. That is to say, excesses are visible around $\Delta \eta_{\mathrm{jj}} \sim 5, m_{\mathrm{jj}}>1 \mathrm{TeV}$, and small $p_{\mathrm{T}}^{H j}$, which all strongly characterize VBF production. The other half of the excess populates the region around BDT score $\sim 0$, meaning that the events do not strongly resemble either VBF or ggF production. Barring the presence of new physics, the most likely explanation for the excess in this BDT score region is the reconstruction of events with one or more pile-up jets, leading to the acceptance of spurious dijet events. In general, it is most likely that these results are the result of statistical fluctuations, in the sense that they are all compatible with the SM expectation within two standard deviations. 


\subsection{Limits on anomalous Higgs boson couplings}

In order to place limits on the presence of anomalous Higgs boson couplings to gauge bosons, a likelihood function is built as a product of conditional probabilities over the event yield $N_{j}$ in each reconstructed event category $j$,

$$
\mathcal{L}(\vec{\kappa}, \vec{\theta})=\prod_{j}^{N_{\text {categories }}} P\left(N_{j} \mid S_{j}(\vec{\kappa}, \vec{\theta})+B_{j}(\vec{\theta})\right) \times \prod_{m}^{N_{\text {nuisance }}} \mathcal{C}_{m}(\vec{\theta})
$$

such that the set of coupling strength parameters $\vec{\kappa}$ represents the POI for a specific hypothesis test. The expected number of signal events $S_{j}(\vec{\kappa}, \vec{\theta})$ is parameterized using the morphing approach described in Section 5.4, with the morphing bases constructed from the samples listed in Section 6.1.1. The test statistic is once again based on a profile likelihood ratio,

$$
q=-2 \ln \frac{\mathcal{L}(\vec{\kappa}, \hat{\vec{\theta}}(\vec{\kappa}))}{\mathcal{L}(\hat{\vec{\kappa}}, \hat{\vec{\theta}}(\hat{\vec{\kappa}}))}=-2 \ln \lambda(\vec{\kappa})
$$

with the conditional (unconditional) maximum-likelihood estimators shown in the numerator (denominator). Several different tests are made using this approach:

- The $\mathcal{C P}$-odd coupling strength $\kappa_{\text {Agg }}$ is measured assuming that all other BSM couplings are equal to zero

- The coupling strengths $\kappa_{H V V}$ and $\kappa_{A V V}$ are probed individually, assuming in each case that all other BSM couplings are equal to zero

- The coupling strengths $\kappa_{H V V}$ and $\kappa_{A V V}$ are probed simultaneously, assuming that all other BSM couplings are equal to zero 


\begin{tabular}{ccccccc}
\hline \hline $\begin{array}{c}\text { BSM coupling } \\
\text { strength } \kappa_{\mathrm{BSM}}\end{array}$ & $\begin{array}{c}\text { Fit } \\
\text { configuration }\end{array}$ & $\begin{array}{c}\text { Expected } \\
95 \% \mathrm{CL}\end{array}$ & $\begin{array}{c}\text { Observed } \\
95 \% \mathrm{CL}\end{array}$ & $\begin{array}{c}\text { Best-fit Best-fit } \\
\hat{\kappa}_{\mathrm{BSM}}\end{array}$ & $\begin{array}{c}\text { Deviation } \\
\hat{\kappa}_{\mathrm{SM}}\end{array}$ & from SM \\
\hline \hline$\kappa_{A g g}$ & $\left(\kappa_{H g g}=\kappa_{\mathrm{SM}}=1\right)$ & {$[-0.47,0.47]$} & {$[-0.68,0.68]$} & \pm 0.43 & - & $1.8 \sigma$ \\
\hline$\kappa_{H V V}$ & $\left(\kappa_{H g g}=\kappa_{\mathrm{SM}}=1\right)$ & {$[-2.9,3.2]$} & {$[0.8,4.5]$} & 2.9 & - & $2.3 \sigma$ \\
$\kappa_{H V V}$ & $\left(\kappa_{H g g}=1, \kappa_{\mathrm{SM}}\right.$ free $)$ & {$[-3.1,4.0]$} & {$[-0.6,4.2]$} & 2.2 & 1.2 & $1.7 \sigma$ \\
\hline$\kappa_{A V V}$ & $\left(\kappa_{H g g}=\kappa_{\mathrm{SM}}=1\right)$ & {$[-3.5,3.5]$} & {$[-5.2,5.2]$} & \pm 2.9 & - & $1.4 \sigma$ \\
$\kappa_{A V V}$ & $\left(\kappa_{H g g}=1, \kappa_{\mathrm{SM}}\right.$ free $)$ & {$[-4.0,4.0]$} & {$[-4.4,4.4]$} & \pm 1.5 & 1.2 & $0.5 \sigma$ \\
\hline \hline
\end{tabular}

Table 9.3: Expected and observed 95\% CL for the $\kappa_{A g g}, \kappa_{H V V}$ and $\kappa_{A V V}$ coupling strengths, the best-fit values of each coupling strength, and the corresponding compatibility with the SM expectation, obtained using negative log-likelihood scans performed with $36.1 \mathrm{fb}^{-1}$ of data at $\sqrt{s}=13 \mathrm{TeV}$. The coupling strength $\kappa_{H g g}$ is fixed to the SM value of one in the fit, while $\kappa_{\mathrm{SM}}$ is either fixed to the SM value of one, or left as a free parameter of the fit.

In each test, the best-fit hypothesis is re-measured, with the likelihood for a given hypothesis test computed relative to its corresponding best-fit measurement. Unless otherwise stated, the SM coupling strength values $\kappa_{\mathrm{SM}}$ and $\kappa_{H g g}$ are fixed to their SM expectations of one. Furthermore, the presence of anomalous couplings is assumed to induce changes only for Higgs boson signal.

The expected and observed test statistic values are shown as functions of one BSM coupling at a time in Figure 9.5, together with the corresponding exclusion limits at $68 \%$ and $95 \%$ confidence levels (CL). The best-fit coupling strength values, and the size of the deviation from the SM, are also summarized in Table 9.3. Worth noting is that the measured event yields in each experimental category do not provide any sensitivity to the sign of the $\kappa_{A g g}$ and $\kappa_{A V V}$ coupling strength parameters. In contrast, the event yields are expected to differ for positive and negative values of $\kappa_{H V V}$, owing to interference effects with the SM $H V V$ interactions detailed in Section 5.3. As a result of the excess number of observed signal events, mainly in the VBF-enriched reconstruction categories, the best-fit values for the coupling strengths $\kappa_{A g g}, \kappa_{H V V}$, and $\kappa_{A V V}$ differ from the expectation of zero, deviating from SM predictions by $1.8 \sigma$, 


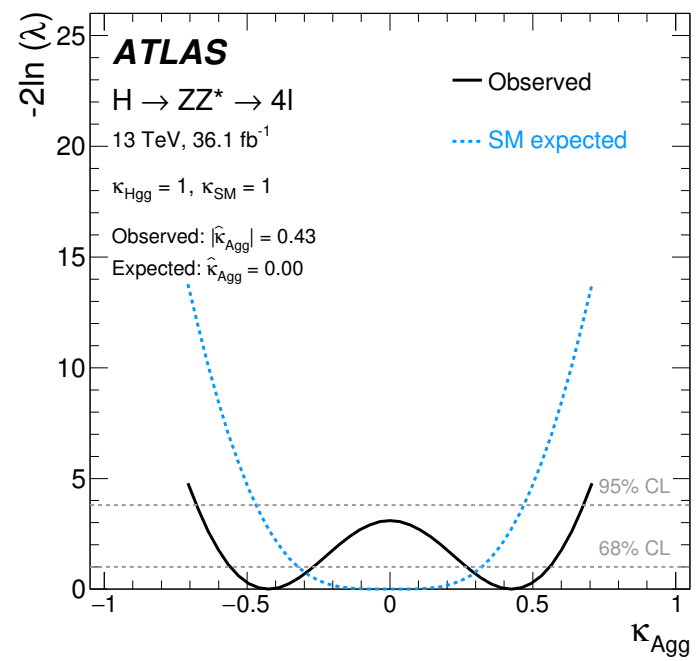

(a) Scan over $\kappa_{A g g}$ with $\kappa_{\mathrm{SM}}, \kappa_{H g g}$ fixed

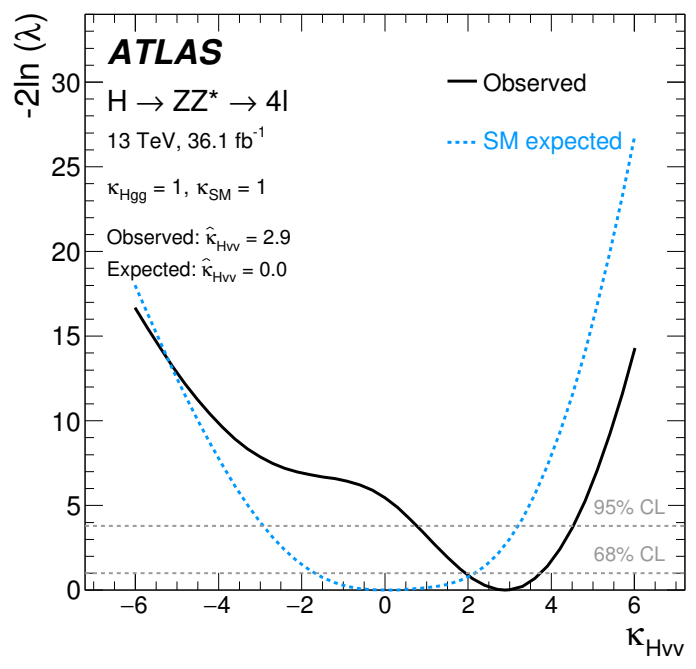

(b) Scan over $\kappa_{H V V}$ with $\kappa_{\mathrm{SM}}$ fixed

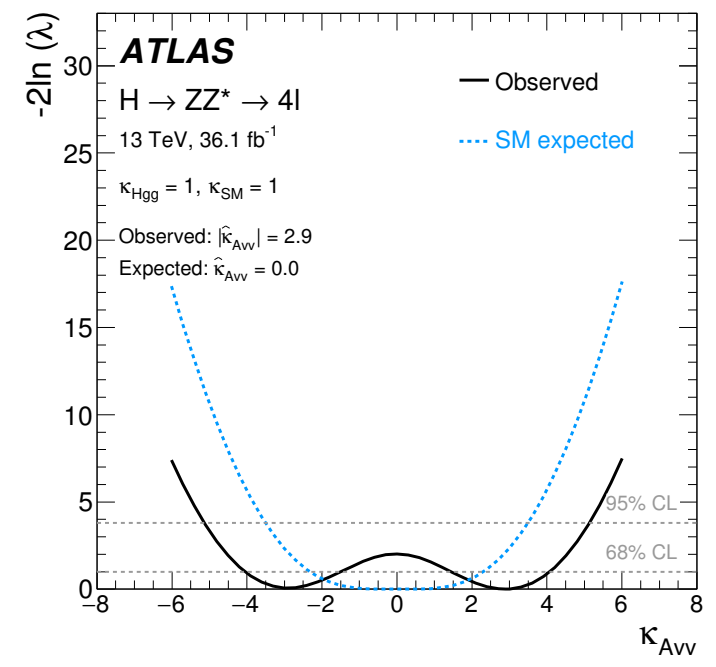

(c) Scan over $\kappa_{A V V}$ with $\kappa_{\mathrm{SM}}$ fixed

Figure 9.5: Observed (solid black line) and SM expected (dashed blue line) negative log-likelihood scans for (a) $\kappa_{A g g}$, (b) $\kappa_{H V V}$ and (c) $\kappa_{A V V}$ coupling strengths using $36.1 \mathrm{fb}^{-1}$ of data at $\sqrt{s}=$ $13 \mathrm{TeV}$. The horizontal lines indicate the value of the test statistic corresponding to the $68 \%$ and 95\% CL intervals for the parameter of interest. 
$2.3 \sigma$, and $1.4 \sigma$, respectively.

Analogous measurements for $\kappa_{H V V}$ and $\kappa_{A V V}$ were also made with the SM coupling strength $\kappa_{\mathrm{SM}}$ left as a free parameter in the fit, which are summarized in Table 9.3. In this scenario, the expected limits on $\kappa_{H V V}$ and $\kappa_{A V V}$ weaken by $15-20 \%$, as the deviations in event yield due to the introduction of BSM couplings can be partially "absorbed" by increasing the SM contribution. Accordingly, the observed exclusion limit of the SM hypothesis decreases to below $2 \sigma(1 \sigma)$ when placing constraints on $\kappa_{H V V}\left(\kappa_{A V V}\right)$ using data, since the observed excess of events is partially absorbed by a $20 \%$ increase of the SM coupling parameter $\kappa_{\mathrm{SM}}$. The best-fit $\kappa_{H V V}$ and $\kappa_{A V V}$ values also decrease correspondingly. Due to interference effects in the $H V V$ vertices under the presence of BSM $\mathcal{C P}$-even couplings, the expected changes in yield for small values of $\kappa_{H V V}$ undershoot those for equivalent values of $\kappa_{A V V}$, so an increase in the value of $\kappa_{\mathrm{SM}}$ does not "absorb" the observed excess to the same degree when constraining $\kappa_{H V V}$. Therefore, the change in the best-fit $\kappa_{H V V}$ value is smaller than that of the best-fit $\kappa_{A V V}$ value, as compared to the fit configuration with $\kappa_{\mathrm{SM}}=1$.

The $\mathcal{C P}$-even and $\mathcal{C P}$-odd BSM Higgs couplings to the weak bosons are also probed simultaneously using the test statistic of Equation ??. The results are shown in the form of two-dimensional 95\% exclusion limit contours in Figure 9.6, and summarized numerically in Table 9.4. The best-fit value of $\hat{\kappa}_{H V V}$ obtained from the $2 \mathrm{D}$ scan is similar to the one obtained in the $1 \mathrm{D}$ scan, while the analogous value of $\hat{\kappa}_{A V V}$ is closer to the SM expectation of zero. A possible reason for this behaviour is that the presence of a non-zero $\kappa_{H V V}$ scales the signal event yields across all reconstruction categories, owing to the non-trivial change in the $4 \ell$ branching ratio. Conversely, a non-zero value of $\kappa_{A V V}$ induces changes mainly in the 1 - and $\geq 2$-jet regions, due 


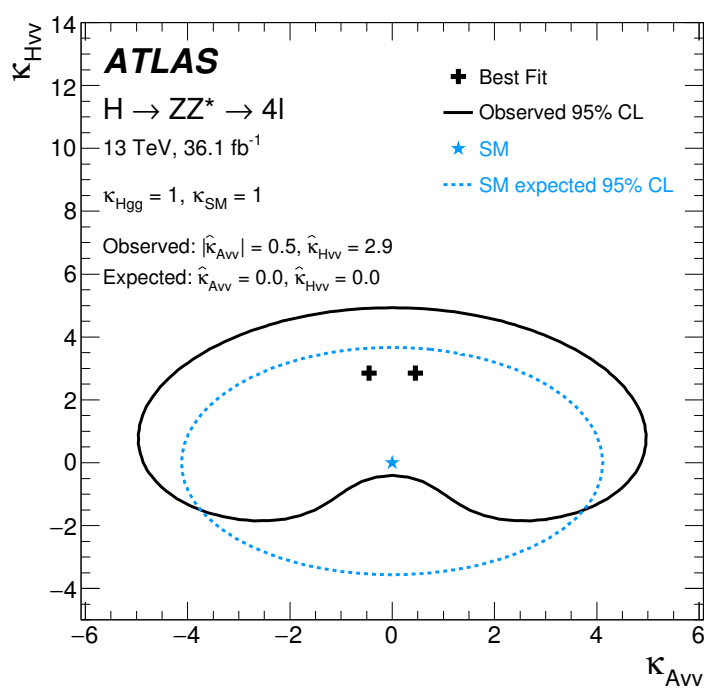

(a) $\kappa_{H V V}$ vs. $\kappa_{A V V}, \kappa_{\mathrm{SM}}$ fixed

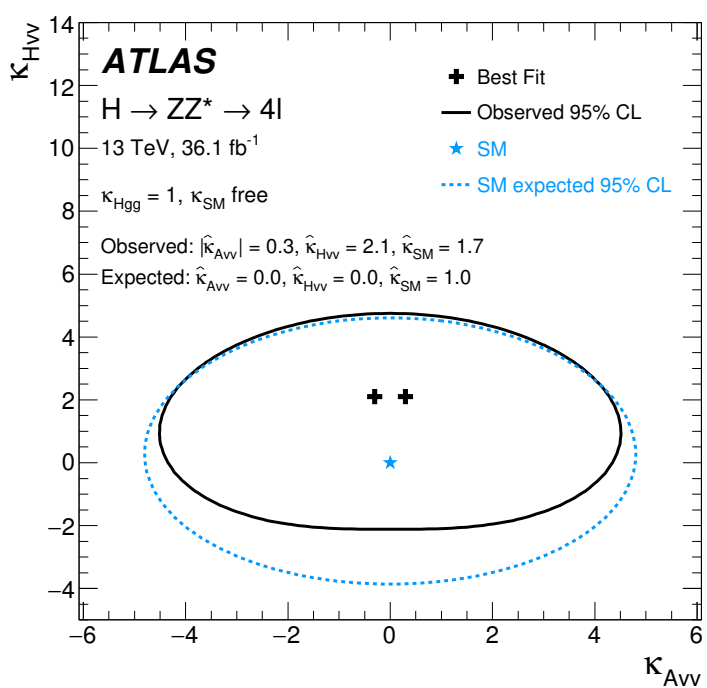

(b) $\kappa_{H V V}$ vs. $\kappa_{A V V}, \kappa_{\mathrm{SM}}$ free

Figure 9.6: Observed (black) and SM expected (blue) contours of the 2D negative log-likelihood at $95 \%$ CL for the $\kappa_{H V V}$ and $\kappa_{A V V}$ coupling strengths with $36.1 \mathrm{fb}^{-1}$ of data at $\sqrt{s}=13 \mathrm{TeV}$. The coupling strength $\kappa_{H g g}$ is fixed to the SM value of one in the fit. The coupling strength $\kappa_{\mathrm{SM}}$ is (a) fixed to the SM value of one or (b) left as a free parameter of the fit.

to the comparatively "slow" change in the decay rate. Therefore, due to the modest excesses in the 0 and 1-jet signal regions, a larger value of $\kappa_{H V V}$ is more favoured by the data, along with a smaller (but non-zero) value of $\kappa_{A V V}$ to account for the residual excess in the $\geq 2$-jet region.

Similar to the one-dimensional scans, two sets of limits are produced, with $\kappa_{\mathrm{SM}}$ being both fixed, and a free parameter in the fit. In both cases, non-zero values of $\kappa_{A V V}$ and $\kappa_{H V V}$ are favoured, although the observed results are still compatible with the SM prediction within $2 \sigma$. Likewise, the degree of compatibility with the SM is unsurprisingly better when $\kappa_{\mathrm{SM}}$ is not fixed. The exclusion limits for $\kappa_{A V V}$ were also found to shrink with a non-fixed $\kappa_{\mathrm{SM}}$, due to the excess of VBF-like events being partially absorbed by the SM contribution. 


\begin{tabular}{c|ccc|c}
\hline \hline Fit configuration & Best-fit $\hat{\kappa}_{H V V}$ & Best-fit $\hat{\kappa}_{A V V}$ & Best-fit $\hat{\kappa}_{\mathrm{SM}}$ & Deviation from SM \\
\hline$\kappa_{H g g}=1, \kappa_{\mathrm{SM}}=1$ & 2.9 & \pm 0.5 & - & $1.9 \sigma$ \\
$\kappa_{H g g}=1, \kappa_{\mathrm{SM}}$ free & 2.1 & \pm 0.3 & 1.7 & $1.2 \sigma$ \\
\hline \hline
\end{tabular}

Table 9.4: The best-fit coupling values and corresponding deviation from the SM expectation, obtained using two-dimensional log-likelihood scans over $\kappa_{H V V}$ and $\kappa_{A V V}$, performed with $36.1 \mathrm{fb}^{-1}$ of data at $\sqrt{s}=13 \mathrm{TeV}$.

\subsection{Outlook for anomalous Higgs boson coupling limits}

This work presents an EFT-based analysis to constrain anomalous couplings in the $H \rightarrow 4 l$ decay channel through direct measurements on data. However, using such an approach to constrain couplings has its own limitations, due to the requirement that any Higgs signal samples undergo simulation to factor in the effects of detector acceptance, efficiency, and resolution, as well as pile-up interactions. Considering the potentially large number of samples necessary to constrain a parameter space made up of more than two or three couplings simultaneously, the use of a direct measurement-based approach can rapidly become impractical due to the underlying computational complexity.

One possible alternative approach is to use unfolded differential cross section measurements to constrain anomalous couplings. Cross section measurements seek to characterize the detector resolution, efficiency, background contributions, pile-up effects, etc. in well-defined fiducial regions, then factor out (or unfold) these elements from the data. The end result of this process is an essentially model-independent measurement of the Higgs signal cross section in a selected region of phase space. The fiducial cross section for some region of phase space is defined as, 


$$
\sigma_{\text {fid }}=\frac{N_{\text {fit }}}{\int \mathcal{L} \times C}
$$

where $N_{\text {fit }}$ is the number of extracted signal events in data, $\int \mathcal{L}$ is the integrated luminosity, and $C$ is a correction factor for detector inefficiency and resolution. A differential cross section distribution further divides the fiducial phase space, binning events in a representative variable (e.g. $N_{j}$ or $\left.p_{\mathrm{T}}^{H}\right)$, such that,

$$
\left(\frac{d \sigma_{\text {fid }}}{d x}\right)_{i}=\frac{N_{\text {fit }}^{i}}{\int \mathcal{L} \times C_{i} \times \Delta x_{i}}
$$

where $N_{\text {fit }}^{i}$ and $C_{i}$ are the extracted number of signal events and correction factor for the $i^{\text {th }}$ bin of the distribution, respectively. The correction term $C_{i}$ in general varies for each bin of the distribution, and is derived on a bin-by-bin basis. Lastly, the term $\Delta x_{i}$ corresponds to the width of the $i^{\text {th }}$ bin, and may be dimensionful.

By using this information, constraints on anomalous couplings would be made by interpreting the unfolded data, rather than by direct coupling measurements. An obvious benefit of this approach is that placing constraints on BSM phenomena would only require the use of truth-level Higgs signal information to compare data to prediction. Furthermore, the critical information needed to make these comparisons is already publicly available; the latest $H \rightarrow Z Z^{*} \rightarrow 4 \ell$ differential cross section measurements using $36.1 \mathrm{fb}^{-1}$ of data recorded at $\sqrt{s}=13 \mathrm{TeV}$ have been reported in Ref. [52], with the differential measurements published to HepDATA ${ }^{1}$, along with the bin-by-bin correction factors and total errors. Accordingly, these measurements can be further used for interpretation without the use of any software specific to ATLAS.

Differential cross sections embed both shape and normalization information into

\footnotetext{
${ }^{1}$ The HePDATA record is available here: https://www.hepdata.net/record/ins1615206
} 
a given distribution, meaning that there is potential to use information from multiple distributions simultaneously to set stricter constraints on anomalous couplings than if one were to use each distribution in isolation. Such an approach was taken by the $H \rightarrow \gamma \gamma$ analysis group, in fact, where limits were set using a multivariate approach based on cross sections from several BSM-sensitive differential distributions [149]. In general, implementing a multivariate fit of this form requires knowledge of the degree of correlation between bins of each differential distribution. For example, the inclusive distribution of $p_{\mathrm{T}}^{H}$ tends to be non-trivially correlated with the jet multiplicity $N_{j}$. So, in order to avoid "double counting" shared information when using both distributions simultaneously, one must factor in the statistical cross correlation between the $i^{\text {th }}$ bin of $p_{\mathrm{T}}^{H}$, and the $k^{\text {th }}$ bin of $N_{j}$ when using them to compute a given test statistic.

The most straightforward way to include statistical cross correlations in a test statistic calculation is through the use of a multivariate $\chi^{2}$,

$$
\chi^{2}(\vec{\kappa})=\Delta x(\vec{\kappa}) \cdot C_{\text {tot }}(\vec{\kappa}) \cdot \Delta x(\vec{\kappa})
$$

where $\Delta x=x_{\text {data }}-x_{\text {pred }}(\vec{\kappa})$ is a vector containing the bin-by-bin differences in cross section between the data and a given hypothesis defined by the vector of coupling strengths $\vec{\kappa}$, and $C_{\text {tot }}(\vec{\kappa})$ is a covariance matrix which quantifies the statistical and systematic relationships between each set of differential measurements. In general, the covariance matrix $C_{\text {tot }}$ can also be a function of the hypothesis vector $\vec{\kappa}$, and is typically written as,

$$
C_{\text {tot }}=C_{\text {stat }}+C_{\text {exp }}+C_{\text {theo }}(\vec{\kappa}),
$$


where $C_{\text {stat }}$ is the statistical covariance matrix, while $C_{\exp }$ and $C_{\text {theo }}(\vec{\kappa})$ quantify the covariances arising from experimental and theoretical systematic uncertainties, respectively.

One particular issue with the $H \rightarrow Z Z^{*} \rightarrow 4 \ell$ channel is that the statistical cross correlations between bins of different variables (i.e. the off-diagonal terms of $\left.C_{\text {stat }}\right)$ are not easily estimated. The $H \rightarrow \gamma \gamma$ analysis group was able to leverage the considerably large non-resonant diphoton background present in the signal region to estimate these correlations in data via bootstrapping [149]. However, the general lack of background in the $4 \ell$ channel means that any attempt at bootstrapping would incur large uncertainties on the derived cross correlations. Therefore, other options must be considered to estimate these correlations, such as modelling them using Monte Carlo, using only single differential distributions, or using distributions made in exclusive regions of phase space, with the latter two options employing statistically uncorrelated measurements, by construction.

To limit the potential theory dependence of the test statistic calculation, a viable option is to use the $Z Z^{*}$ differential distributions of $p_{\mathrm{T}}^{H}$ made in bins of $N_{j}=0,1$, and $\geq 2$ jets, shown in Figure 9.7, which were measured in the latest $4 \ell$ differential cross section analysis [52]. The distribution of $p_{\mathrm{T}}^{H}$ in the $\geq 2$-jet bin, in particular, allows for greater sensitivity to anomalous Higgs boson couplings to the $W^{ \pm}$and $Z$ bosons, as the $p_{\mathrm{T}}^{H}$ spectrum is expected to change quite significantly in VBF and VH production for strongly non-SM hypotheses (as first seen in Section 5.3). The spectra in the 0and 1-jet bins allow for greater statistical precision, and constraints on changes to the overall signal normalization. Worth noting is that the final differential bin for the $N_{j}=0$ distribution is only an upper limit, and as such, should be excluded from 


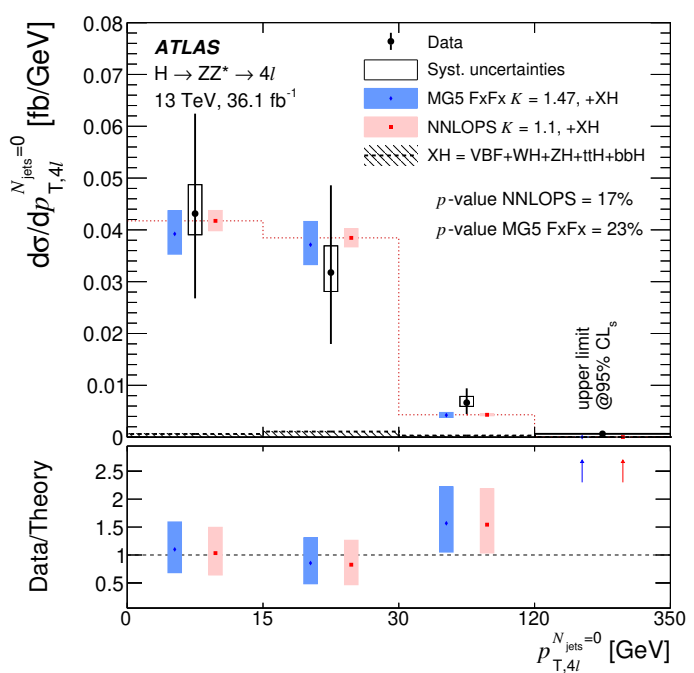

(a) $p_{\mathrm{T}}^{H}, N_{j}=0$

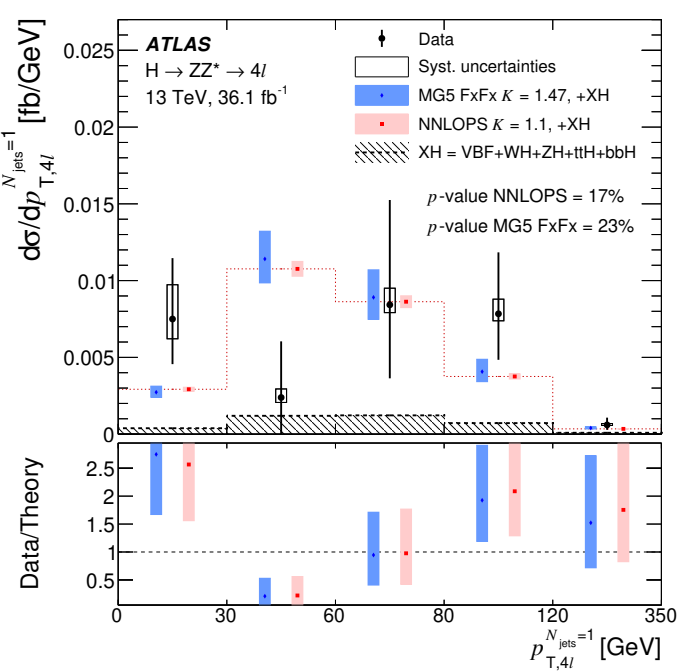

(b) $p_{\mathrm{T}}^{H}, N_{j}=1$

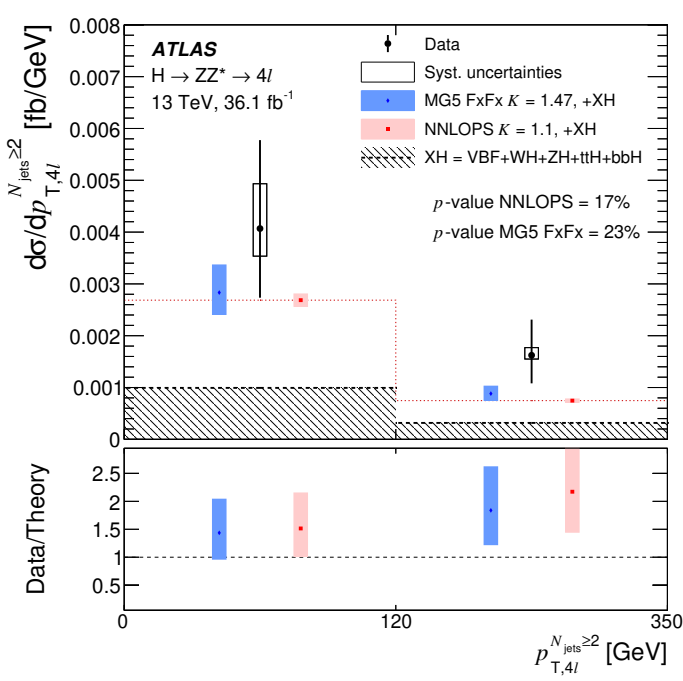

(c) $p_{\mathrm{T}}^{H}, N_{j} \geq 2$

Figure 9.7: Unfolded differential cross sections of $p_{\mathrm{T}}^{H}$ in bins of $N_{j}=0,1$, and $\geq 2$ jets, made using $36.1 \mathrm{fb}^{-1}$ of $H \rightarrow Z Z^{*} \rightarrow 4 \ell$ data recorded at $\sqrt{s}=13 \mathrm{TeV}$ [52]. 


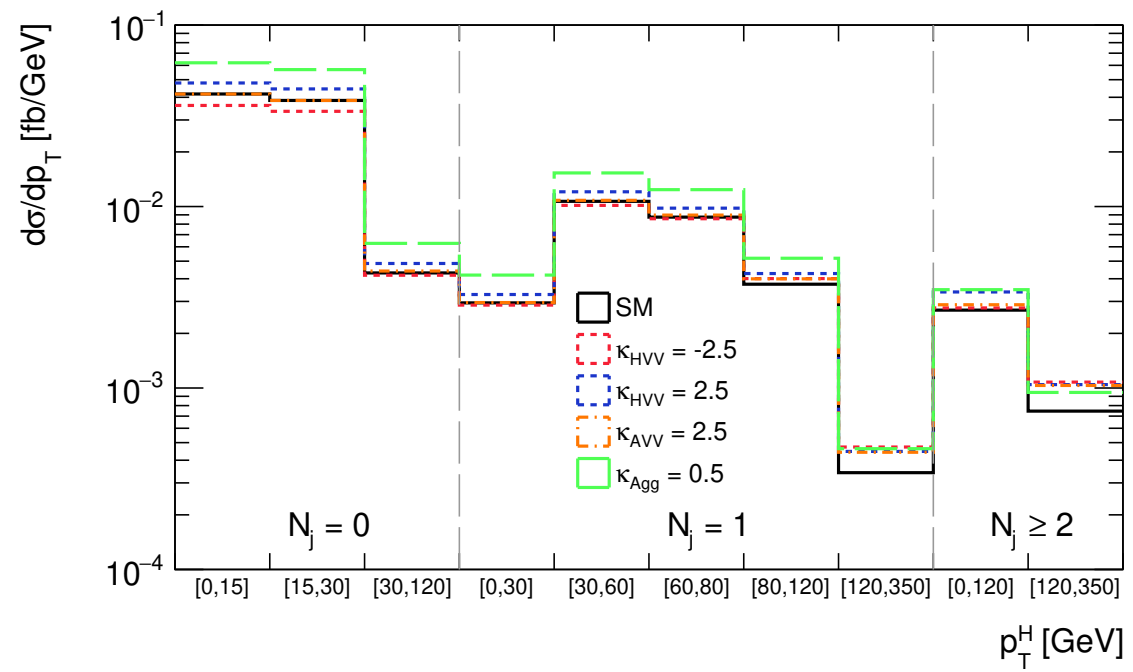

Figure 9.8: Comparison of $Z Z^{*} \mathrm{SM}$ differential cross section predictions of $p_{\mathrm{T}}^{H}$ vs. $N_{j}$ at $\sqrt{s}=$ $13 \mathrm{TeV}$ with a number of BSM hypotheses, derived using the Higgs Characterization model.

further consideration, leaving 10 total measurements to be used across all jet bins.

To evaluate the potential efficacy of this approach, initial tests were made using truth-level Monte Carlo, generated via MADGrAPH5_aMC@NLO in a fashion identical to Section 6.1.1. The best prediction scaling of Section 6.7 was also applied for each differential bin to ensure that the MC predictions converged to the best-known SM expectations with no anomalous couplings present. The truth-level events were further subjected to the selection cuts of Sections 6.3 and 6.4, with the exception of any reconstruction-level cuts on object quality (e.g. vertex fit, track quality requirements, etc.), which are not applicable to truth-level information. The allowed $4 \ell$ invariant mass range is also extended to $115<m_{4 l}<130 \mathrm{GeV}$ in the differential analysis. In order to efficiently perform hypothesis tests for arbitrary points in the coupling parameter space, the histogram morphing approach described in Section 5.4 was used to interpolate between BSM hypotheses. A comparison of the differential cross section predictions for the SM and various BSM hypotheses is given in Fig- 
ure 9.8, illustrating the effect of non-zero $\kappa_{H V V}, \kappa_{A V V}$, and $\kappa_{A g g}$ on the differential $p_{\mathrm{T}}^{H}$ spectra.

The test statistic chosen for this evaluation is a $\Delta \chi^{2}$ between two hypotheses namely, the alternate (BSM) and null (SM) scenarios,

$$
\Delta \chi^{2}=\chi_{\mathrm{BSM}}^{2}-\chi_{\mathrm{SM}}^{2}=\chi^{2}(\vec{\kappa})-\chi^{2}(\overrightarrow{0})
$$

where $\chi_{\mathrm{BSM}}^{2}$ is the $\chi^{2}$ value obtained from testing a given BSM hypothesis, and $\chi_{\mathrm{SM}}^{2}$ is computed with respect to the SM hypothesis. In both scenarios, the $\chi^{2}$ values are evaluated using predictions generated from the morphed truth-level Monte Carlo samples, with the tests applied to MC-derived pseudo-data to produce expected limits.

Because the total errors for each differential bin - including statistical and systematic error - are already available from the HEPDATA archive, the derivation of the covariance matrix $C_{\text {tot }}$ simplifies as follows,

$$
C_{\text {tot }}=\operatorname{diag}\left(\left(\Delta \sigma_{\text {data }, i}^{\text {fid }}\right)^{2}\right)+C_{\text {theo }}^{\text {BSM }}
$$

where $C_{\text {theo }}^{\mathrm{BSM}}$ is a theoretical uncertainty-based covariance matrix which varies with the BSM hypothesis being tested. This term is introduced to account for theoretical uncertainties which are not related to the unfolding, but rather the division of the BSM cross section predictions into exclusive bins of $p_{\mathrm{T}}^{H}$ and $N_{j}$, with the ggF component in particular being susceptible to the IR-sensitivity effects discussed in Section 8.2.1. The prescriptions of Sections 8.2.1 and 8.2.2 are used to calculate scale variation / bin migration and PDF uncertainties which enter into $C_{\text {theo }}^{\mathrm{BSM}}$ as, 


$$
C_{\text {theo }}^{\mathrm{BSM}, i j} \propto C_{\mathrm{PDF}}^{\mathrm{SM}, i j}+C_{\mathrm{QCD}}^{\mathrm{SM}, i j}
$$

with the important distinction that the magnitudes of the covariance terms scale with the predicted BSM yields, i.e. the covariance between bins $i$ and $j$ is expressed as,

$$
C_{\text {theo }}^{\mathrm{BSM}, i j}=\frac{N_{i}^{\mathrm{BSM}} N_{j}^{\mathrm{BSM}}}{N_{i}^{\mathrm{SM}} N_{j}^{\mathrm{SM}}}\left(C_{\mathrm{PDF}}^{\mathrm{SM}, i j}+C_{\mathrm{QCD}}^{\mathrm{SM}, i j}\right) .
$$

Each $C_{\text {theo }}^{\mathrm{BSM}, i j}$ term is computed separately for $\mathrm{ggF}$ and $\mathrm{VBF}+\mathrm{VH}$ production, with the final covariance matrix being a linear sum of the covariance matrices for each production type.

For a simple $\chi^{2}$ test across $n$ uncorrelated measurements, the test statistic shape is a $\chi^{2}$ distribution with $n$ degrees of freedom. This distribution is used to compute the $p$-value (hence, exclusion limits) for an arbitrary hypothesis, given the $\chi^{2}$ value with respect to the observed data. For a $\Delta \chi^{2}$-based method, however, the test statistic distributions for the null and alternate hypotheses - defined as $f\left(\Delta \chi^{2} \mid\right.$ null $)$ and $f\left(\Delta \chi^{2} \mid\right.$ alt $)$, respectively - typically vary with the alternate hypothesis being tested. The shape of the test statistic distribution for the alternate hypothesis is used to compute the $p$-value, as described in Ref. [131]. This value determines whether said hypothesis is excluded by the data, as illustrated in Figure 9.9. In particular, the value of $p_{\text {alt }}$ is computed as,

$$
p_{\text {alt }}=\int_{\Delta \chi_{\text {obs }}^{2}}^{\infty} f\left(\Delta \chi^{2} \mid \text { alt }\right) d\left(\Delta \chi^{2}\right) .
$$

Given an observed test statistic $\Delta \chi_{\text {obs }}^{2}$, a value of $1-p_{\text {alt }}>0.68$ implies the hypothesis is excluded at the $68 \% \mathrm{CL}$ (or $1 \sigma$ ) with respect to the null hypothesis, while $1-p_{\text {alt }}>$ 


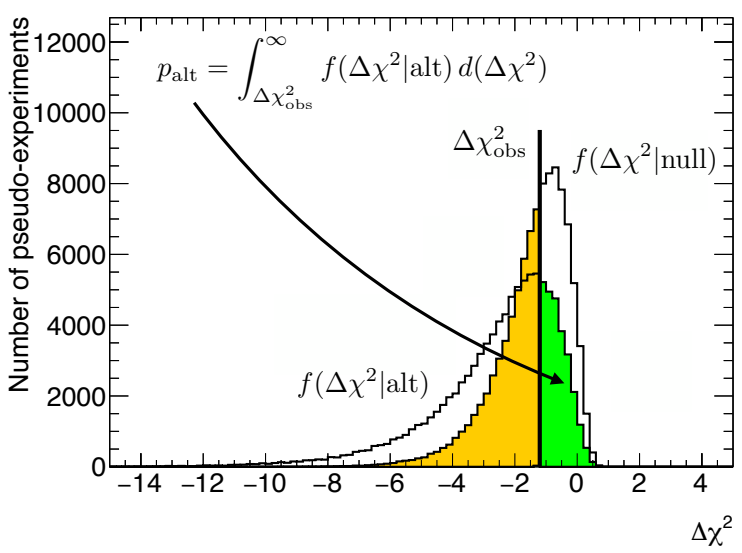

Figure 9.9: Example of $\Delta \chi^{2}$ test statistic distributions for null and alternate hypotheses, along with the definition of the $p$-value $p_{\text {alt }}$.

0.95 implies the hypothesis is excluded at $95 \%$ confidence (or $2 \sigma$ ).

As the test statistic distributions for the null and alternate hypotheses are frequently not known analytically, they can be derived explicitly using ensembles of pseudo-experiments (or toys), in which the nominal predictions of a given hypothesis are varied in a manner constrained by their associated statistical and systematic uncertainties, as well as any underlying correlations. A sufficiently large ensemble of toys should span the breadth of all possible permutations of the nominal predictions, yielding a shape and range of the test statistic distribution for that hypothesis. By choosing $p_{\mathrm{T}}^{H}$ measurements in exclusive bins of $N_{j}$, each differential cross section is statistically independent with respect to the others, allowing such correlations to be neglected in the toy ensembles. For an integrated luminosity of $36.1 \mathrm{fb}^{-1}$, the differential cross section predictions correspond to event yields of 10 or less signal events per bin, implying that the event yields vary in a manner governed by Poisson statistics.

To properly factor in the Poissonian nature of the statistical uncertainty when creating ensembles of pseudo-experiments, the toy generation is performed using a three-step process, depicted in Figure 9.10. In the first step, the nominal signal event 


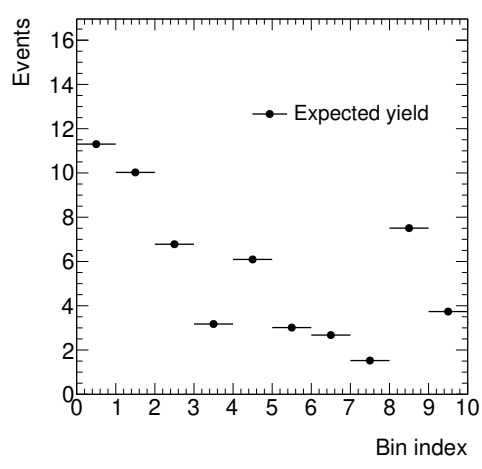

(a) Expected yields

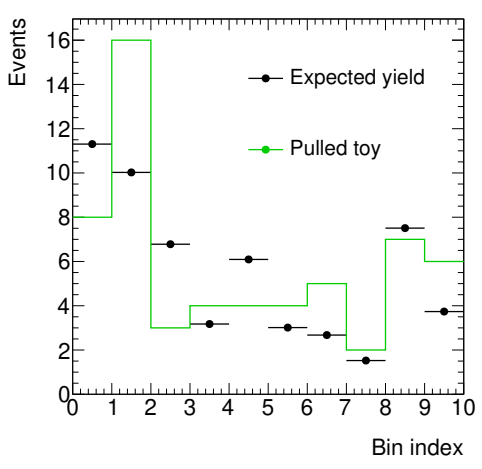

(b) Toy generation

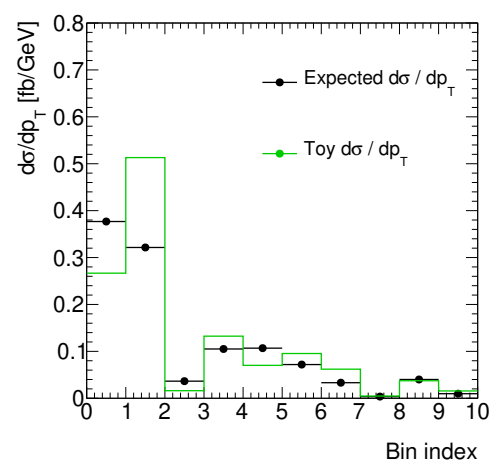

(c) Differential cross sections

Figure 9.10: Example of the method used to generate toys (pseudo-experiments) from expected differential cross sections, which are later used to generate $\Delta \chi^{2}$ test statistic distributions.

yield for bin $k$ of a given hypothesis is computed by scaling the $k^{\text {th }}$ differential cross section prediction by its corresponding bin width, correction factor, and the integrated luminosity, essentially inverting the result of Equation 9.3. The $k^{\text {th }}$ predicted event yield $N_{k}$ is then "pulled" by replacing it with a Poisson-distributed random number $N_{k}^{\prime}$, where the mean of the Poisson distribution has $\lambda_{k}=N_{k}$. Lastly, the $k^{\text {th }}$ pulled event yield is converted back into a differential cross section using the integrated luminosity, correction factor, and bin width for the $k^{\text {th }}$ bin.

Each systematic uncertainty source is also allowed to vary the yield in the $k^{\text {th }}$ bin upward or downward, which is done in a correlated fashion across each bin. In particular, the $j^{\text {th }}$ systematic uncertainty source pulls the $k^{\text {th }}$ event yield $N_{k}$ as,

$$
\left(N_{k}\right)^{\prime}=N_{k}+r_{N} \times \Delta N_{k}^{j},
$$

where $r_{N}$ represents a random number drawn from the standard Normal distribution, and $\Delta N_{k}^{j}$ is the absolute uncertainty due to the $j^{\text {th }}$ systematic uncertainty source. The total pull on the $k^{\text {th }}$ prediction due to systematic uncertainty is then, 


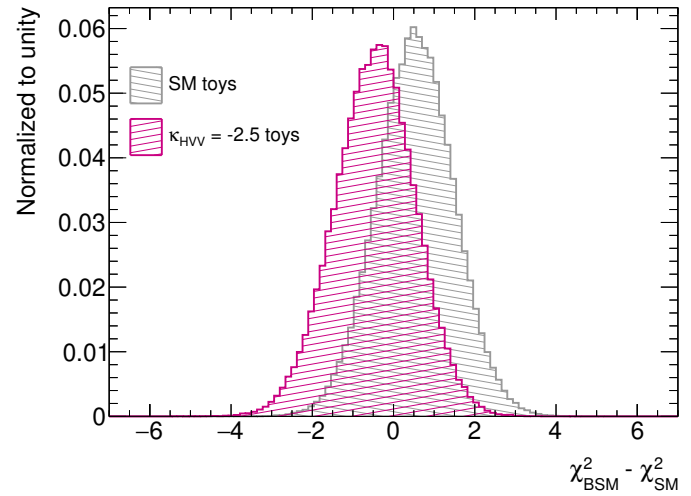

(a) Testing $\kappa_{H V V}=-2.5$

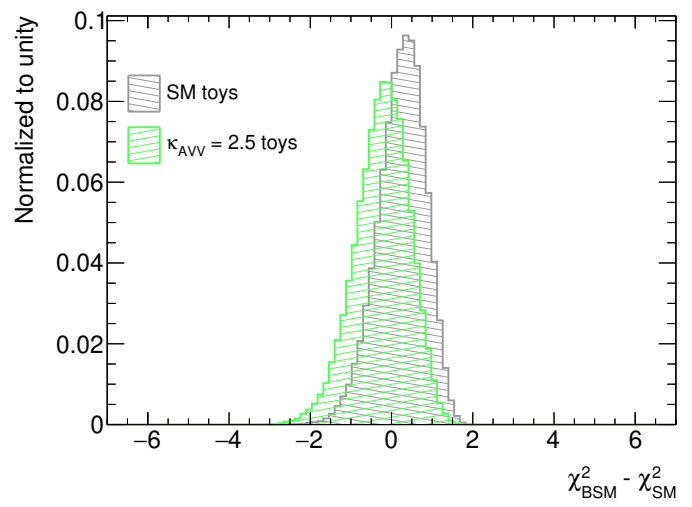

(c) Testing $\kappa_{A V V}=2.5$

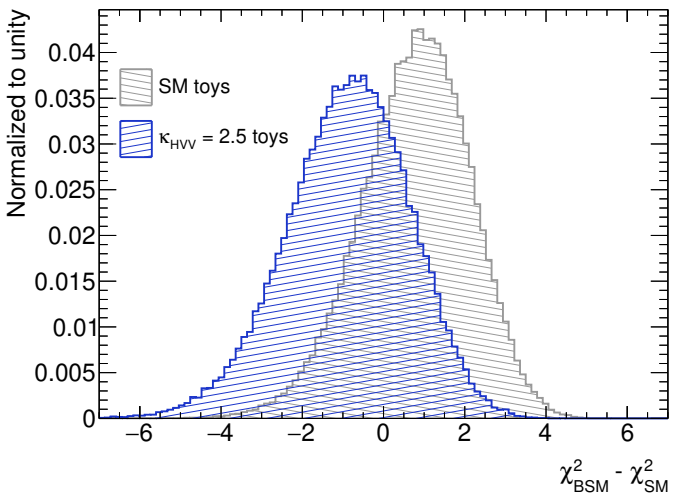

(b) Testing $\kappa_{H V V}=2.5$

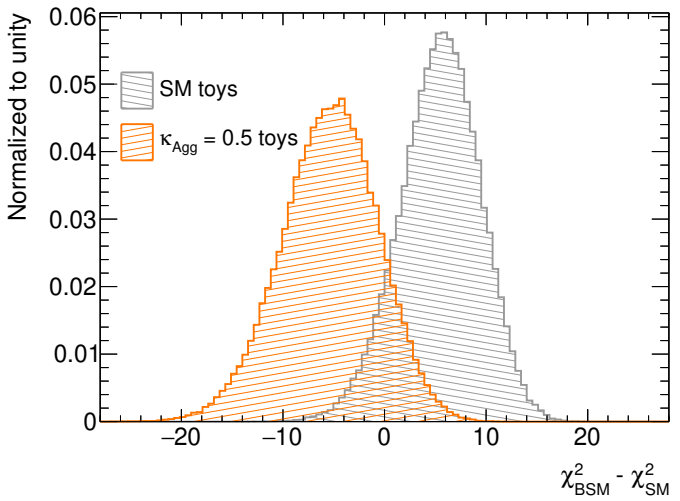

(d) Testing $\kappa_{A g g}=0.5$

Figure 9.11: Distributions of $\Delta \chi^{2}=\chi_{\mathrm{BSM}}^{2}-\chi_{\mathrm{SM}}^{2}$ for various hypothesis tests, computed using toys centred around the SM hypothesis, as well as toys centred around different BSM hypotheses.

$$
\left(N_{k}\right)^{\prime}=N_{k}+\sum_{j=1}^{n_{\mathrm{syst}}} r_{N}^{j} \times \Delta N_{k}^{j}
$$

It is also worth mentioning that the notation $r_{N}^{j}$ implies that for the $j^{\text {th }}$ uncertainty source, all differential bins are pulled by the same random number, owing to the fact that the deviations due to a particular systematic uncertainty are taken to be $100 \%$ correlated across all bins. 
Examples of the $\Delta \chi^{2}$ test statistic distributions generated from ensembles of toys are shown in Figure 9.11, where 100k toys are generated around both the null (SM) and alternate (BSM) hypotheses in each example. To create these distributions, every toy from both ensembles is subjected to the $\Delta \chi^{2}$ calculation of Equation 9.7, with the resultant $\Delta \chi^{2}$ value used to populate the corresponding histogram. The behaviour of these test statistic distributions reflects the phenomenological effects of introducing anomalous couplings in Higgs production and decay, as first observed in Section 5.3. For example, differences in the $\Delta \chi^{2}$ distributions are observed for $\kappa_{H V V}=-2.5$ and 2.5 , since the expected changes to $\sigma \times \mathcal{B}$ induced by this coupling are asymmetrical about zero. Furthermore, the test statistic distributions for $\kappa_{A g g}=0.5$ show a much greater degree of separation than those with anomalous couplings solely in the $H V V$ vertices, since the presence of $\kappa_{A g g} \neq 0$ induces a global increase in the ggF cross section. The effects induced by non-zero $\kappa_{A V V}$ and $\kappa_{A g g}$ are also symmetrical about zero, hence the test statistic distributions for negative values of these coupling strengths will be essentially the same as for positive values.

Using $\Delta \chi^{2}$ distributions of the form shown in Figure 9.11, expected exclusion limits relative to the $\mathrm{SM}$ can be set on anomalous $\mathcal{C P}$-even and odd couplings in the Higgs boson production and decay vertices. To set these expected limits, the lower limit of the integral in Equation 9.11 is taken to be the median $\Delta \chi^{2}$ value of the SM toys for a given BSM hypothesis test. To evaluate the robustness of this limit setting approach, two scenarios were considered:

1. A simultaneous constraint on $\mathcal{C P}$-odd and even $H V V$ coupling strengths $\kappa_{A V V}$ and $\kappa_{H V V}$, and

2. Constraints on $\mathcal{C P}$-odd interactions in Higgs production and decay, i.e. varying 
$\kappa_{A g g}$ and $\kappa_{A V V}$ simultaneously

For both scenarios, the SM coupling strengths $\kappa_{\mathrm{SM}}$ and $\kappa_{H g g}$ were fixed to their nominal values of one. Therefore, the first scenario is also analogous to the $2 \mathrm{D}$ scan results in Figure 9.6a), meaning that the expected limits can be compared to those of the direct measurement approach, as well. The expected exclusion limits for both cases are shown in Figure 9.12 in the form of $2 \mathrm{D}$ contour analyses. The results of Figure 9.12a), in particular, show a good agreement with the expected limits of Figure 9.6a), indicating that, in this scenario, the interpretation of differential cross section measurements provides equivalent sensitivity to the direct measurement approach for the constraint of anomalous couplings. The distribution of Figure 9.12b) shows an almost rectangular relationship between the two coupling strengths, which may be attributed to the fact that $\kappa_{A g g}$ affects only ggF Higgs production, while $\kappa_{A V V}$ affects only $\mathrm{VBF}+\mathrm{VH}$ production. Therefore, the confidence limits for non-zero $\kappa_{\text {Agg }}$ and $\kappa_{A V V}$ are driven largely by the change in the ggF production rate.

Differential cross section measurements currently exist across multiple Higgs decay channels and centre-of-mass energies in ATLAS, so the ultimate goal of this approach would be to combine these individual measurements using one large, multivariate fit. For example, the $\sqrt{s}=8$ and $13 \mathrm{TeV}$ measurements from $H \rightarrow \gamma \gamma$ and $H \rightarrow 4 \ell$ channels $[52,150,151,152]$ may be concatenated into a single vector, with the corresponding covariance matrix built from the statistical and systematic uncertainties associated to each individual measurement. The primary benefit of such an approach is that cross section information from different decay channels and $\sqrt{s}$ values is statistically independent by construction, since no information is "shared" between measurements. Furthermore, systematic uncertainties can generally be treated as fully 


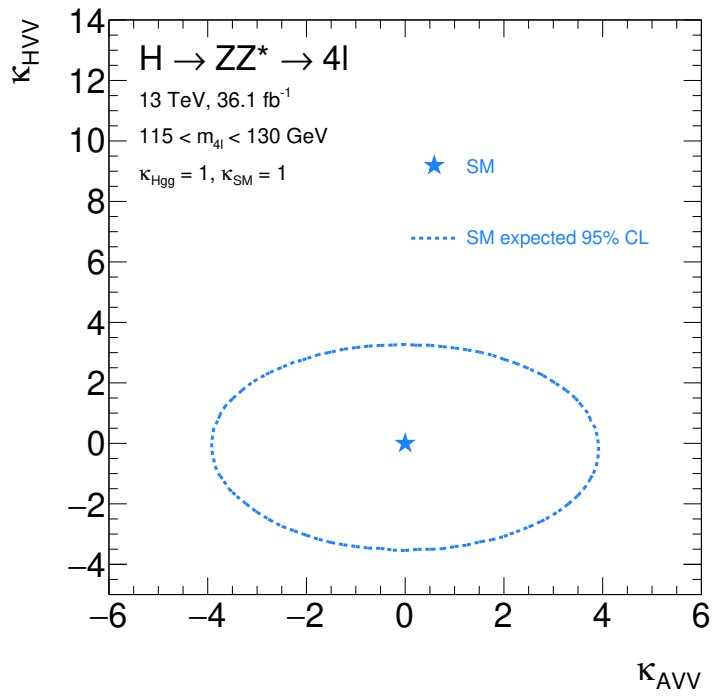

(a) $\kappa_{H V V}$ vs. $\kappa_{A V V}$

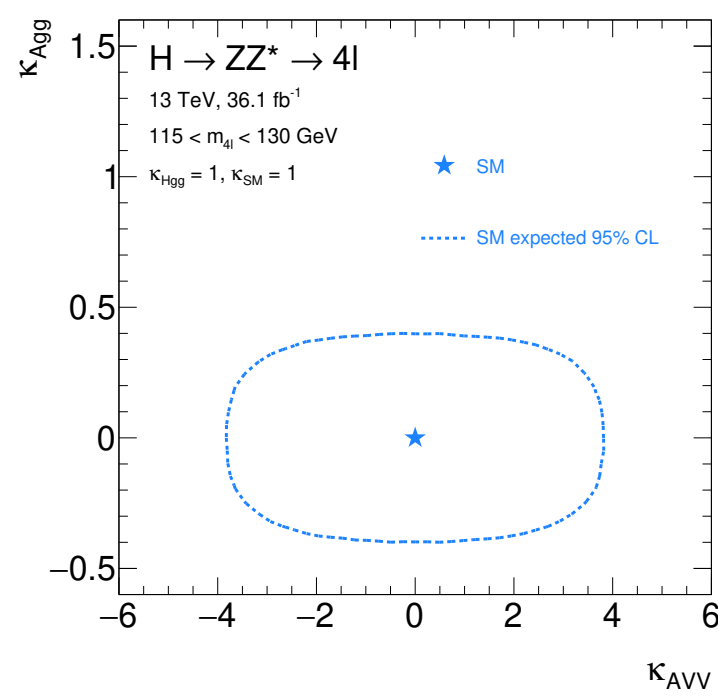

(b) $\kappa_{A g g}$ vs. $\kappa_{A V V}$

Figure 9.12: Expected exclusion limits on anomalous couplings in the Higgs Characterization model, set using differential cross section pseudo-data generated around the SM at $\sqrt{s}=13 \mathrm{TeV}$. Shown are simultaneous constraints on BSM $\mathcal{C} \mathcal{P}$-even and odd coupling strengths $\kappa_{H V V}$ and $\kappa_{A V V}$ (left), and constraints on $\mathcal{C P}$-odd coupling strengths $\kappa_{A g g}$ and $\kappa_{A V V}$ (right). 
correlated across different $\sqrt{s}$ and decay channels, simplifying the construction of these elements of the covariance matrix. Although such results are currently outside the scope of this work, the development of such a combination is active and ongoing, with the hope of inclusion in a future differential cross section analysis. 


\section{Chapter 10}

\section{Summary}

Analyses have been presented which measure the signal strengths and couplings of the Higgs boson in the $H \rightarrow Z Z^{*} \rightarrow 4 \ell$ decay channel using $36.1 \mathrm{fb}^{-1}$ of $p p$ collision data recorded at $\sqrt{\mathrm{s}}=13 \mathrm{TeV}$ using the ATLAS detector during 2015 and 2016. The Higgs boson candidate events were categorized into several topologies meant to increase sensitivity to different production modes, as well as various regions of phase space. The vector boson fusion signal strength measurement, in particular, made use of a multivariate discriminant which was optimized to distinguish between vector boson fusion and gluon fusion-mediated Higgs boson production. Similar discriminants were used in other experimental categories to improve the sensitivity to different Higgs production modes, and reject experimental background.

Using this approach, a deviation of $2.2 \sigma$ was observed for vector boson fusionmediated Higgs production due to an observed excess of events characterized by the presence of at least two jets, and a dijet invariant mass above $120 \mathrm{GeV}$. Otherwise, it was found that the signal strength measurements for each Higgs boson production mode were generally in agreement with Standard Model predictions. Based on 
the observed event yields in each reconstructed event category, constraints were also placed on possible Beyond Standard Model interactions of the Higgs boson within the framework of the Higgs Characterization model, which extends the Standard Model to include a number of common Beyond Standard Model interactions. Exclusion limits were set on $\mathcal{C P}$-even and $\mathcal{C} \mathcal{P}$-odd Beyond Standard Model couplings of the Higgs boson to vector bosons, and on $\mathcal{C P}$-odd Higgs boson couplings to gluons. In each case, the data were shown to be consistent with the Standard Model hypothesis, with the Standard Model hypothesis deviating by roughly $2 \sigma$ from the best-fit hypothesis, due to the observed excess of vector boson fusion-like events.

A new ATLAS electron and photon reconstruction algorithm has also been presented, wherein electron and photons are built using dynamically-sized clusters. The algorithm further identifies clusters which have emerged from material interactions with the Inner Detector, and combines them with the primary clusters to form "superclusters". The performance of this algorithm has been evaluated for single particles with and without pile-up interactions, and for a number of electron-related physics topologies. The algorithm generally shows good improvement in terms of energy resolution with respect to the previous $e / \gamma$ reconstruction algorithm. For electrons and converted photons, improvements in energy resolution of $30-40 \%$ are possible using superclusters, with the largest improvements found for low $E_{\mathrm{T}}$ particles, and for clusters reconstructed in the end-cap calorimeter. These resolution improvements translate directly to mass resolution improvements of $5-10 \%$ in $Z$ and $J / \psi$ decays to $e^{+} e^{-}$pairs, as well as $H \rightarrow Z Z^{*} \rightarrow 4 \ell$ decays featuring electrons.

The performance improvements seen for electrons and converted photons are also maintained for scenarios with higher pile-up, as a result of topo-cluster noise thresh- 
olds being adjusted to suit the luminosity conditions of a given data taking period. The move to high-luminosity conditions may require a re-tuning of the cell summation approach for unconverted photons, as well as the development of an average pile-up subtraction approach to compensate for increased average noise levels. The derivation of a pile-up-sensitive multivariate calibration may also allow for an even more robust energy response against a wide array of luminosity conditions. 


\section{Appendix A}

\section{Personal contributions to the ATLAS collaboration}

The following is a summary of the author's specific contributions to the measurements and results presented in this thesis, and the broader ATLAS experiment:

1. Multivariate SM VBF and BSM studies in the $H \rightarrow Z Z^{*} \rightarrow 4 \ell$ channel

$\triangleright$ Relevant chapters: Chapters 5, 7, and 8

$\triangleright$ Summary of contributions: I was the developer of a multivariate classifier used to isolate VBF Higgs boson production in the $H \rightarrow 4 l$ channel for the analysis of the full 2015+2016 ATLAS data set. I was also responsible for optimizing the technique to minimize the theoretical uncertainties on the gluon fusion cross section induced by the use of a multivariate classifier. The derivation and validation of the multivariate classifier are detailed in Chapter 7. These elements were incorporated into the larger analysis 
which measured the signal rates of different Higgs boson production modes in the $H \rightarrow 4 l$ channel.

I was responsible for the generation and validation of Monte Carlo samples of ggF-mediated $H \rightarrow 4 \ell$ events featuring anomalous couplings in the Higgs production and decay vertices. Furthermore, I contributed to the development and validation of tools used to interpolate between BSM hypotheses in an effective field theory (morphing), and used these tools to identify a region of coupling space in which to search for evidence of $\mathcal{C P}$-odd ggF-mediated Higgs boson production. Each of these elements are detailed in Chapter 5, and were incorporated into the analysis to set limits on the presence of anomalous couplings in the $H \rightarrow 4 l$ channel.

For both the SM and BSM-focused $H \rightarrow 4 l$ analyses, I was responsible for estimating the theoretical uncertainties associated to the ggF-mediated $H \rightarrow 4 \ell$ signal rates. These uncertainties included scale variation / bin migration effects emerging from the division of the ggF cross section into exclusive 0, 1, and $\geq 2$-jet regions, along with PDF and shower Monte Carlo generator variations, as detailed in Chapter 8. I was a primary author and editor of documentation describing each analysis, which was adapted into an ATLAS public note (ATLAS-CONF-2017-043), and is part of an ATLAS paper submitted to JHEP. 
2. Supercluster-based electron, photon reconstruction

$\triangleright$ Relevant chapter: Chapter 4

$\triangleright$ Summary of contributions: I was the principal developer of a new electron reconstruction algorithm in ATLAS which uses dynamic clustering to build "superclusters". This approach improves the energy resolution of electrons by recovering photons radiated in the Inner Detector due to bremsstrahlung. I also contributed to the development of the corresponding electron calibration, and validated the performance of the algorithm for different physics topologies using specially-derived Monte Carlo samples. The algorithm was later adapted for use with photons and photon conversions, and the larger method has now become the default electron/photon reconstruction algorithm for ATLAS. I was also the principal author of an ATLAS public document detailing the algorithm and its performance (ATL-PHYS-PUB-2017-022). 
3. Prospects for VBF $H \rightarrow Z Z^{*} \rightarrow 4 \ell$ measurements at the HL-LHC

$\triangleright$ Relevant chapter: Appendix D

$\triangleright$ Summary of contributions: I was the principal analyzer responsible for implementing a Monte Carlo-based analysis which used multivariate techniques to evaluate the potential visibility of $\mathrm{VBF} H \rightarrow 4 \ell$ signal at the High-Luminosity LHC after acquiring $3 \mathrm{ab}^{-1}$ of data at $\sqrt{s}=14 \mathrm{TeV}$. I was also responsible for running the statistical analysis and assessing theoretical uncertainties associated to the use of a multivariate discriminant in the analysis. I also served as the primary author of an ATLAS public note summarizing the results (ATL-PHYS-PUB-2016-008), which was incorporated into the ATLAS Phase-II Scoping Document (CERN-LHCC-2015-020). 


\section{Appendix B}

\section{Higgs, jet kinematic definitions}

This appendix provides a handy reference guide defining some kinematic quantities of interest common to several chapters in this work. Natural units are employed with $c=1$ and $e=1$, allowing representations of energy $(E)$, momentum $(p)$, and mass $(m)$ in units of $\mathrm{eV}$.

\begin{tabular}{lc}
\hline \hline Quantity & Definition \\
\hline$\eta$ & $-\ln (\tan (\theta / 2))$ \\
$p_{\mathrm{T}}$ & $|\vec{p}| / \cosh (\eta)$ \\
$E_{\mathrm{T}}$ & $\sqrt{m^{2}+p_{\mathrm{T}}^{2}}$ \\
$\Delta \eta_{\mathrm{jj}}$ & $\left|\eta_{j_{1}}-\eta_{j_{2}}\right|$ \\
$\Delta \phi_{j j}$ & $\left|\phi_{j_{2}}-\phi_{j_{1}}\right| \bmod 2 \pi$ \\
$\Delta R$ & $\sqrt{(\Delta \eta)^{2}+(\Delta \phi)^{2}}$ \\
$m_{\mathrm{jj}}$ & $\sqrt{\left(E_{j_{1}}+E_{j_{2}}\right)^{2}-\left(\vec{p}_{j_{1}}+\vec{p}_{j_{2}}\right)^{2}}$ \\
$\eta_{Z Z}^{*}$ & $\left|\left(\eta_{j_{1}}+\eta_{j_{2}}\right) / 2-\eta_{Z Z}\right|$ \\
\hline \hline
\end{tabular}




\section{Appendix $\mathrm{C}$}

\section{Merging scale choice and jet}

\section{kinematics for ggF FxFx samples}

To evaluate the effect that the choice of merging scale $\mu_{q}$ has on the single jet and dijet kinematics of NLO-merged ggF events, three samples of NLO-merged ggF $H \rightarrow 4 \ell$ events were generated using the FxFx merging process in MADGRAPH5_aMC@NLO, and showered using Pythia8, with the Higgs boson decaying as $H \rightarrow Z Z^{*} \rightarrow 4 \ell$. Each sample was subject to the same $4 \ell$ candidate selection, and subsequent jet selection, as described in Section 6. Each sample is generated using 150k events before $4 \ell$ candidate selection. Plots are shown inclusive to their respective minimum necessary jet multiplicity; for example, the Higgs boson $p_{\mathrm{T}}$ and pseudorapidity are shown inclusively, while all leading jet quantities require at least one jet with $p_{\mathrm{T}}>30 \mathrm{GeV}$, and all subleading jet require at least two jets with $p_{\mathrm{T}}>30 \mathrm{GeV}$. All dijet quantities shown require two jets with $p_{\mathrm{T}}>30 \mathrm{GeV}$, as well as a minimum dijet mass of $m_{\mathrm{jj}}>40 \mathrm{GeV}$.

The resultant plots are shown in Figure C.0 for choices of $\mu_{q}=30,40$, and 50 
$\mathrm{GeV}$. Within this range of merging scale, it is found that the kinematic results are generally consistent within the bin-by-bin MC statistical uncertainties. Though there are bin-by-bin fluctuations of upwards of $40 \%$ in some distributions, the associated MC statistical error tends to be large enough to only deviate within $1 \sigma$ of the prediction for $\mu_{q}=30 \mathrm{GeV}$. Therefore, it can be said that, for this particular range of values tested, the choice of merging scale does not have a meaningfully large effect on the Higgs boson or jet kinematics. 

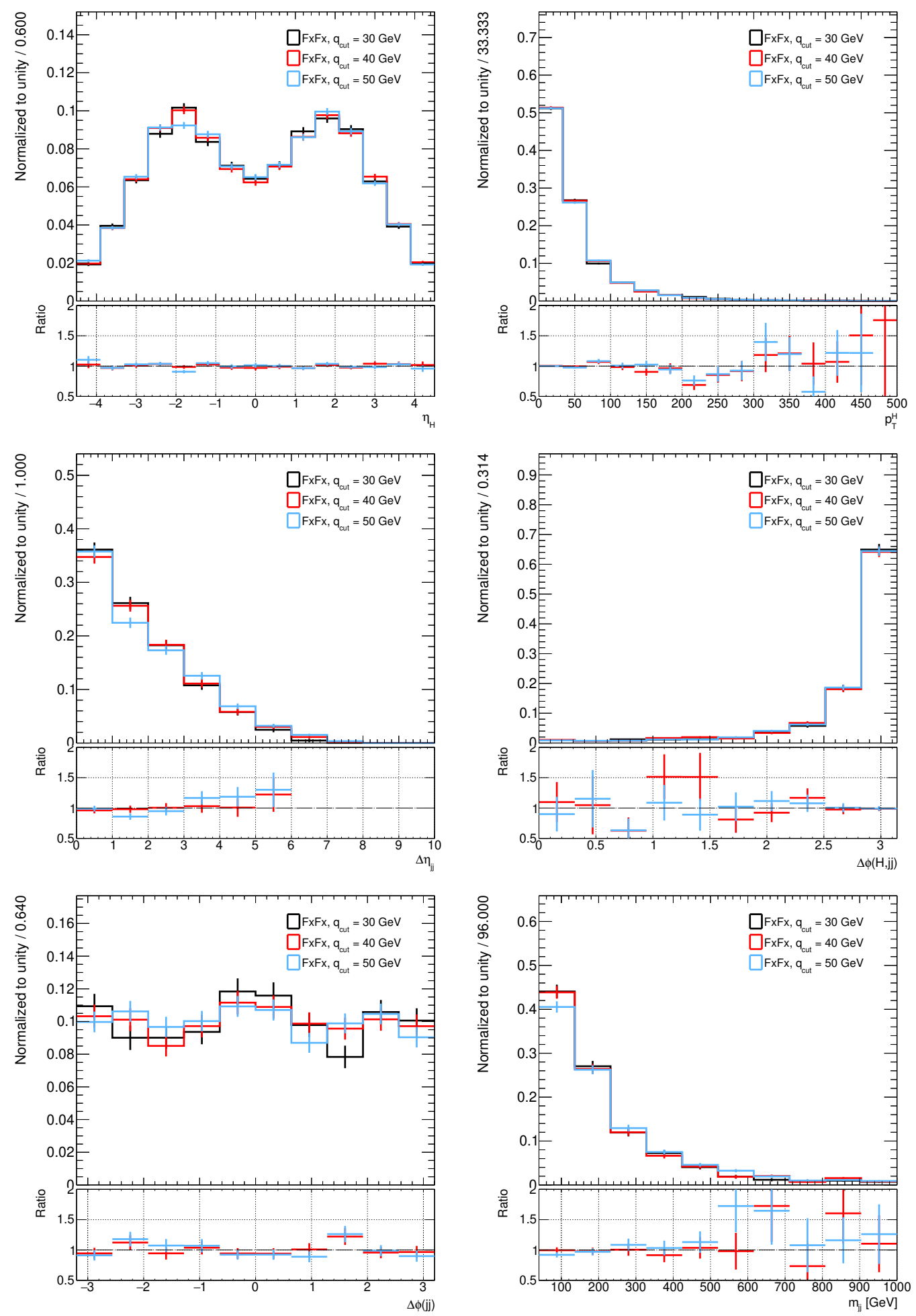

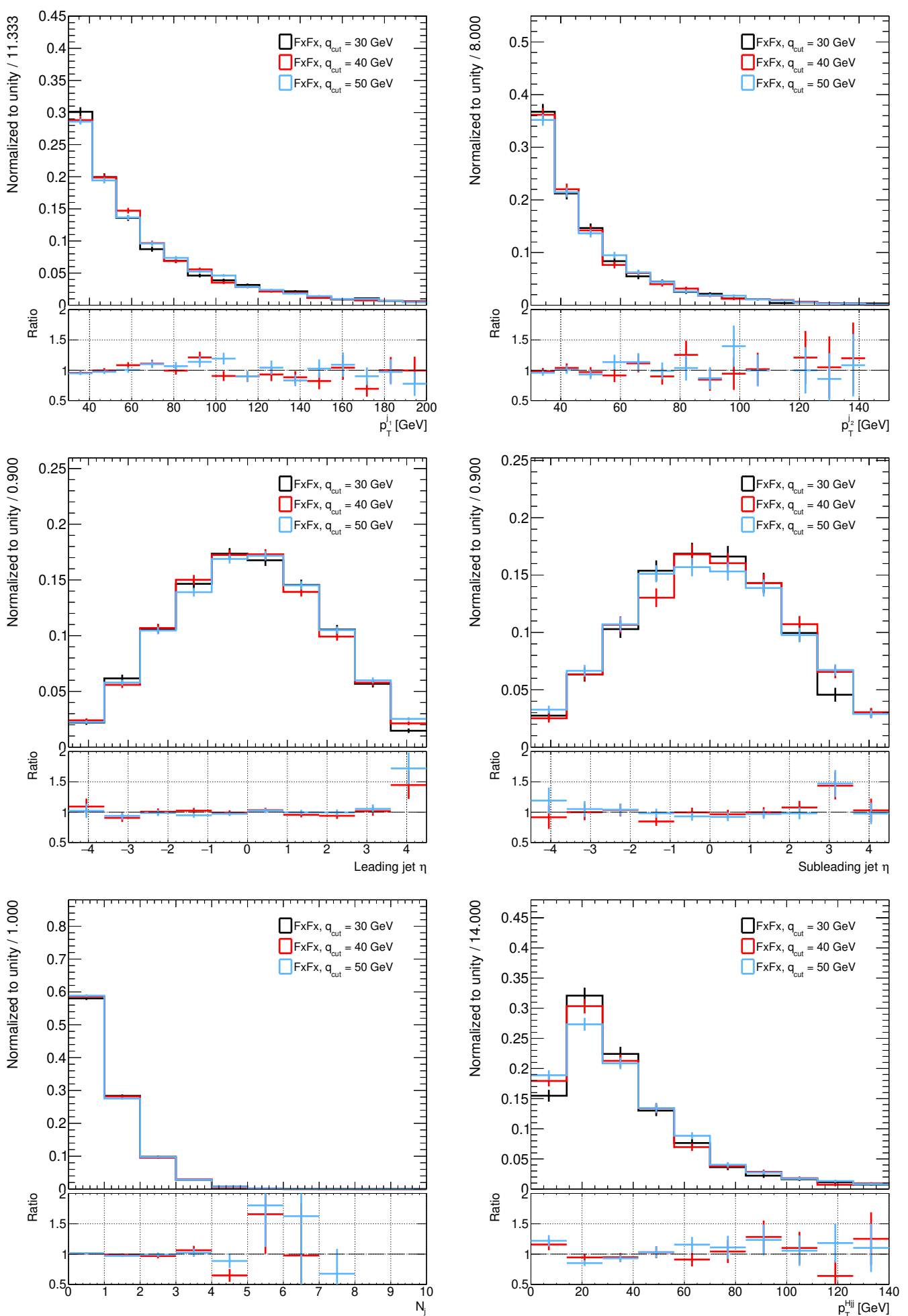

Figure C.0: Comparison of relevant Higgs boson and jet kinematic quantities for samples of NLOmerged ggF events generated using the FxFx merging process in MADGraPH5_aMC@NLO. Results are shown for choices of $\mu_{q}=30,40$, and $50 \mathrm{GeV}$. 


\section{Appendix D}

\section{VBF $H \rightarrow 4 \ell$ prospects at HL-LHC}

The High-Luminosity LHC (HL-LHC) is an extension of the LHC physics program past 2026, at which point ATLAS is expected to have collected approximately $300 \mathrm{fb}^{-1}$ of data. This milestone will be preceded by a second long shutdown period between 2019 and 2020, wherein several necessary upgrades to the LHC injector and cryostat system will be made to bring the centre-of-mass energy of the LHC to $\sqrt{s}=14 \mathrm{TeV}$. Over a ten year period, the HL-LHC physics program will exploit this higher operating energy to acquire roughly ten times the amount of data acquired by 2026, producing $3000 \mathrm{fb}^{-1}$ of data at $\sqrt{s}=14 \mathrm{TeV}$. To accomplish such a goal, Phase-II upgrades for the HL-LHC will take place during 2024 - 2025. In this scenario, the accelerator will operate with a peak levelled luminosity of $\mathcal{L} \approx 7.5 \times 10^{34} \mathrm{~cm}^{-2} \mathrm{~s}^{-1}$, which corresponds to $\mu \approx 200$ [153].

At high-luminosity conditions, a particularly important physics goal is the continued sensitivity to rarer production and decay modes which feature couplings to the weak bosons, such as VBF-mediated Higgs boson production, and $H \rightarrow Z Z^{*} \rightarrow 4 \ell$ decays. While the Higgs boson production rates will increase with the greater $\sqrt{s}$ and 
luminosity, so too will the amount of in-time and out-of-time pile-up interactions for each recorded event. Furthermore, at these luminosity conditions, the gluon fusion production cross section will increase proportionately, thus continuing to be another source of contamination in the VBF-like phase space.

Therefore, ensuring that weak-boson-mediated Higgs signals are sufficiently free of pile-up and background contamination will be crucial for improving constraints on Higgs boson couplings at high $\sqrt{s}$, as well as placing improved constraints on BSM physics. With regard to isolating VBF production, these effects will be particularly prominent in the forward region $(|\eta| \geq 2.5)$, for which ATLAS currently has no tracking capability. In this regard, an upgraded tracking detector which extends into the forward region will be an important element in maintaining the visibility of VBF-mediated Higgs signal against pile-up. In this appendix, prospective results are presented which evaluate the effects of different ATLAS detector upgrade scenarios on the visibility of VBF-mediated $H \rightarrow 4 \ell$ signal at the HL-LHC, both against pile-up, as well as contamination from ggF-mediated $H+2 j$ signal.

\section{D.1 The HL-LHC and Phase-II upgrades}

At the extraordinary levels of luminosity expected from the HL-LHC, the proposed Phase-II detector upgrades are targeted towards rejecting the high amounts of pile-up expected, while still affording the ability to trigger quickly and efficiently on physically interesting interactions [153]. As a matter of nomenclature, the "best-case" (but also highest cost) scenario in terms of proposed upgrades is defined as the Reference scenario, which is designed to maximize physics performance - and discovery potential - during the running of the HL-LHC. Two alternative, lower-cost configurations are 
also proposed, referred to as the Middle and Low scenarios. Accordingly, the changes in scope for the Middle and Low scenarios will affect the ability to trigger on, and reconstruct, leptons, jets, $b$-jets, and MET at high $\langle\mu\rangle$.

In particular, the changes to the ATLAS detector for the HL-LHC upgrade broadly encompass the trigger system, the Inner Detector, the end-cap and forward calorimeter systems, as well as the Muon Spectrometer. The changes to the trigger system are comprised of faster, custom triggering hardware; in the Reference design scenario, the $\mathrm{L} 1$ triggers operate at rates up to $400 \mathrm{kHz}$, while the Middle and Low scenarios reduce the rate of the $\mathrm{L} 1$ trigger to $200 \mathrm{kHz}$. In addition, a new L1 track trigger encompasses the full coverage of the tracking system, while the HLT system includes an upgraded tracker trigger, as well as improved EF processors. With regards to the inner tracking system, the Reference scenario brings in an improved Strip tracker, and crucially, extends the coverage of the Pixel detector to $|\eta|<4$.0. The Middle scenario limits the end-cap coverage of the Strip tracker, and also reduces the extension of the Pixel detector to $|\eta|<3.2$. Lastly, the Low scenario implements only a very limited form of the Strip tracker in the first barrel layer, while limiting the coverage of the Pixel detector to a range of $|\eta|<2.7$.

In all scenarios, the readout electronics of the LAr and Tile calorimeters will require upgrades to improve radiation tolerance, and compensate for the higher trigger rates expected from the upgraded ATLAS trigger system. Furthermore, in the Reference scenario, the calorimeter system includes a finely-segmented timing detector covering the region $2.4<|\eta|<4.3$, meant to improve calorimeter response in a region which in general has a lower degree of segmentation compared to the central region of $|\eta|<2.5$. In the Middle and Low scenarios, however, no upgrades of the detectors 
in the end-cap and forward region are generally implemented.

The changes the Muon Spectrometer largely come in the form of new readout electronics, along with chamber replacements, to allow for the MS to cope with the increased trigger rates expected at the HL-LHC. In the Reference scenario, tracking coverage will also be extended to the forward region through the addition of micropattern gaseous or silicon pixel detectors between the end-cap calorimeters and the NSWs, spanning $2.7<|\eta|<4$.0. However, this high- $\eta$ extension is not present for Middle and Low scenarios.

In the Reference scenario, the MDT readout electronics will also be adapted to the $400 \mathrm{kHz}$ trigger rate and increased hit rates, along with the read-out electronics chains in the barrel and end-cap regions. To maintain a reasonable level of trigger efficiency, new RPCs will be installed in the inner layer of the barrel muon spectrometer, while new, high resolution TGC chambers will replace the present chambers in the inner ring of the Big Wheel. In the Middle scenario, the number of chambers which receive upgraded electronics is reduced, with the Low scenario in particular not containing any upgrade of the RPC and MDT chambers. Conversely, the Reference, Middle, and Low scenarios all require the same upgrade for the TGC detectors.

\section{D.2 Higgs boson signal, background simulation}

As these upgrades pertain to the observation of the VBF $H \rightarrow 4 \ell$ process, it is necessary to evaluate how well the planned forward calorimeter and tracking system upgrades perform in each scenario. The main goal for each scenario is to understand the degree of mitigation of pile-up that the upgrades confer, e.g. through the association of "true" forward jets to the primary hard-scattering vertex through use of 
tracking information, and conversely, through the rejection of pile-up jets which do not meet the same criteria. This section describes the methods by which simulated Higgs boson signal and background samples are produced, and how the expected detector and luminosity conditions for the HL-LHC are recreated using simulation.

\section{D.2.1 Higgs boson signal samples}

In this analysis, hard-scattering events for VBF-mediated Higgs boson production were produced using the POWHEG framework, with parton showering performed using Pythia8. The VBF signal Monte Carlo samples were produced using the VBFH generator, which is a complete implementation of VBF Higgs-boson production at NLO QCD. Since the contribution of hadronic VH-mediated Higgs production in the dijet region $m_{\mathrm{jj}}>120 \mathrm{GeV}$ is almost negligible, for the purposes of this study, this signal process was neglected, along with smaller contributions from ttH and leptonic VH-mediated Higgs production.

A primary concern when performing any study of VBF-mediated Higgs boson production is the adequate modelling of the large "background" contribution of ggFmediated Higgs boson signal, produced in association with two jets. As reported in the combined results for the $H \rightarrow Z Z^{*} \rightarrow 4 \ell$ channel at $\sqrt{s}=7$ and $8 \mathrm{TeV}$ [129], the contamination by dijet gluon fusion events in the VBF-enriched region is roughly $44 \%$. Therefore, in order to properly discriminate this large source of contamination, this process must be well-modelled theoretically. To this end, the Powheg HJJ generator was chosen to model this signal contribution, which provides ggF $H+2 j$ calculations at NLO QCD. The use of a higher-order matrix element calculation serves two crucial purposes, in that it provides a better estimate of the process cross 
section, and the modelling of the third subleading jet is done within the matrix element calculation, as opposed to coming from the parton showering stage. The presence of this additional radiation in the matrix element improves the modelling of

quantities that are correlated with the presence of a third jet, such as $p_{\mathrm{T}}^{H j j}$ or $\eta_{Z Z}^{*}$, first defined in Section 7.3.

\section{D.2.2 Background samples}

A small, but non-zero, source of background in this $H \rightarrow 4 \ell$ analysis comes from the irreducible $q q \rightarrow Z Z^{*}+X$ process, where $X$ represents 2 or more additional jets. This source of background is generally more prominent in events with lower jet multiplicities, as $\geq 2$-jet events make up only $\sim 10 \%$ of the background yield. In order to account for this contribution, truth Monte Carlo $q q \rightarrow Z Z^{*}+X$ events were simulated at $\sqrt{s}=14 \mathrm{TeV}$ using the SHERPA MC generator and included as a source of background in the analysis. Smaller contributions from reducible $t \bar{t}$ and $Z+$ jets background sources were not considered, as their contributions to the total background are at least an order of magnitude smaller than that of the $q q Z Z$ process.

\section{D.2.3 Truth object smearing and pile-up simulation}

To emulate the effects of reconstructing particles within the upgraded ATLAS detector, the four momenta of the truth physics objects relevant to this analysis (jets, electrons, and muons) were subjec to energy and momentum smearing, with the smearing factors derived using techniques developed for physics studies presented at the ECFA 2013 [154] and 2014 [155] workshops. This resolution smearing was achieved using $\eta$ and $p_{\mathrm{T}}$-dependent smearing functions derived based on detailed simulations 
of each ATLAS upgrade scenario. In addition to the resolution smearing, simulated reconstruction-level pile-up jets were also mixed into the smeared Higgs boson signal and background samples on an event-by-event basis. The presence of these additional jets was meant to simulate the expected jet multiplicities and kinematic distributions of high pile-up conditions for both $\langle\mu\rangle=140$ and 200 .

The tools used for this analysis also simulated tracking confirmation for jets, which uses tracking information to distinguish jets emerging from the primary vertex, and those originating from pile-up interactions. As described in Section D.1, separate tracking confirmation configurations were used for the Reference, Middle, and Low scenarios. For the Low scenario, jet tracking confirmation only extends to $|\eta|<2.4$, which is functionally the same range as the current ATLAS Inner Detector tracking system. The primary vertex jet tracking confirmation was simulated as a function of jet $p_{\mathrm{T}}$ and $|\eta|$, with anywhere from $80 \%$ to $100 \%$ of jets being kept for a given event. Conversely, the simulated track confirmation was tuned to fix the pile-up jet efficiency at $2 \%$, meaning that only $2 \%$ of generated pile-up jets were randomly kept. The tracking efficiency cuts were only applied to jets with $p_{\mathrm{T}}<100 \mathrm{GeV}$, and only within the $|\eta|$ regions containing tracker instrumentation. Accordingly, the overall degree of pile-up rejection was expected to differ for each layout.

In practice, the ATLAS reconstruction software provides only a unified collection of jets to analyzers, which can contain objects from both the primary vertex, and pile-up interactions. Therefore, the smeared truth jets and simulated pile-up jets were merged into a single collection using several steps. Firstly, the four momenta of the truth-level jets from the hard scattering interaction had $p_{\mathrm{T}}$ and $\eta$-based smearing factors applied to simulate detector response, then were subject to the kinematic 
selection described in Table D.1. A uniformly distributed random number between 0 and 1 was assigned to each jet to determine whether it passed the tracking efficiency cut. Secondly, a random number of simulated pile-up jets were generated. The initial number of pile-up jets generated was sampled from a Poisson distribution with its mean set to the average number of pile-up jets expected for $\langle\mu\rangle=140$ or $\langle\mu\rangle=200$ at $\sqrt{s}=14 \mathrm{TeV}$. The jet kinematics were also sampled from template distributions of $p_{\mathrm{T}}$ and $\eta$, derived using samples of simulated VBF $H \rightarrow \mu \mu$ events generated at $\sqrt{s}=14 \mathrm{TeV}$ which have passed through a full detector simulation. The final state of this particular process has a well-defined topology (two muons, two light jets), allowing the hard scattering process to be "extracted" from each event, producing samples of pure simulated pile-up jets.

After generating the collection of simulated pile-up jets, each jet was assigned a uniformly-distributed random number between 0 and 1, such that a given pile-up jet was immediately rejected if this assigned random number was larger than the tracking efficiency cut. The simulated pile-up jets which passed the tracking efficiency cut were then required to pass the same jet selection cuts as the hard scatter jets. Once each step was complete, the two collections of jets were merged, and the resultant collection was sorted in order of descending $p_{\mathrm{T}}$, to emulate the order in which the jets would appear in an actual reconstructed event.

\section{D.2.4 BDT training and variable shapes}

To evaluate the impact of HL-LHC operating conditions on the separation of VBF and $\operatorname{ggF} H \rightarrow 4 \ell$ signal, the BDT-based analysis methodology of Chapter 7 was extended to the prospects study. In order to account for differences in detector response and 
tracking coverage between scenarios, the BDT separating VBF and ggF production was re-trained for each of the Reference, Medium, and Low scenarios, as well as $\langle\mu\rangle=200$ and 140 conditions. Variables that strongly characterize the presence of a third subleading jet, such as $\Delta \mathrm{R}_{j Z}^{\min }$ and $\eta_{j_{3}}^{*}$, were dropped from consideration, as it was found that for both VBF and ggF production, the third jet came most frequently from simulated pile-up, rather than the hard scattering process. Otherwise, the BDT was trained and used in accordance with the optimization studies of Chapter 7 .

Comparisons of the kinematics of the BDT training variables are given in Figure D.1 for VBF events, and Figure D.2 for ggF events. It is clear from these plots that the presence of high pile-up leads to a significant degradation of purity for the ggF signal. To some extent, this is not a surprising result; because only $\sim 12 \%$ of ggF events have $\geq 2$ jets, then the introduction of one or more pile-up jets leads to a significant amount of "fake" ggF-mediated $H+2 j$ events passing the selection cuts, even in the most optimistic reconstruction scenario. Worth noting about the shape of $\Delta \eta_{\mathrm{jj}}$, in particular, is that "spikes" emerge in the distribution at the point where tracking coverage ends, demonstrating the necessity of extending the tracking coverage to the forward region, in order to mitigate the effects of high pile-up as much as possible.

In contrast to the ggF process, the majority of VBF signal is composed of events with two hard, forward jets. Therefore, this signal is expected to be more robust against the presence of additional pile-up jets, which should ideally manifest as a closer resemblance of the Reference scenario to the scenario with zero pile-up. Such behaviour seems to be reflected in the shapes of the BDT training variables, which generally do not deviate strongly from the distributions shown without pile-up. No- 
table exceptions are seen in the shapes of the subleading jet $p_{\mathrm{T}}$ and $p_{\mathrm{T}}^{H j j}$, which appear to be more strongly influenced by the presence of pile-up jets.

\section{D.3 Analysis methodology}

As this study seeks to evaluate the prospective performance of the VBF $H \rightarrow 4 \ell$ analysis at the HL-LHC, the analysis methodology involves both the selection of candidate $4 \ell$ events, as well as the use of techniques which separate VBF from ggF Higgs boson production.

\section{D.3.1 Event selection}

The four lepton candidate selection was applied to electrons and muons, and followed the prescription of the combined $\sqrt{s}=7$ and $8 \mathrm{TeV} H \rightarrow Z Z^{*} \rightarrow 4 \ell$ analysis [129]. The list of selection cuts applied is provided in Table D. $1^{1}$. The expected number of $\mathrm{VBF}$ and $\mathrm{ggF}$ hard scattering-only events after kinematic and dijet selection at $\mathcal{I}=3000 \mathrm{fb}^{-1}$, scaled to the process cross sections expected for $\sqrt{s}=14 \mathrm{TeV}$, are given Table D.2. In the combined $\sqrt{s}=7$ and $8 \mathrm{TeV}$ analysis [129], the signalto-background ratio was $S / B \sim 7$ for the VBF-enriched category, where $S$ is the total signal yield from all Higgs boson production mechanisms, and the background $B$ included $q q Z Z, t \bar{t}$, and $Z+$ jets events. Within this subset of events, dijet gluon fusion events served as a significant contaminant, making up $\sim 44 \%$ of the yield for the 7 and $8 \mathrm{TeV}$ combined results. Therefore, the focus of this analysis was directed towards separating dijet VBF and gluon fusion events.

\footnotetext{
${ }^{1}$ The $\sqrt{s}=13 \mathrm{TeV}$ analysis selection, although more up-to-date, was not finalized when this study was made.
} 

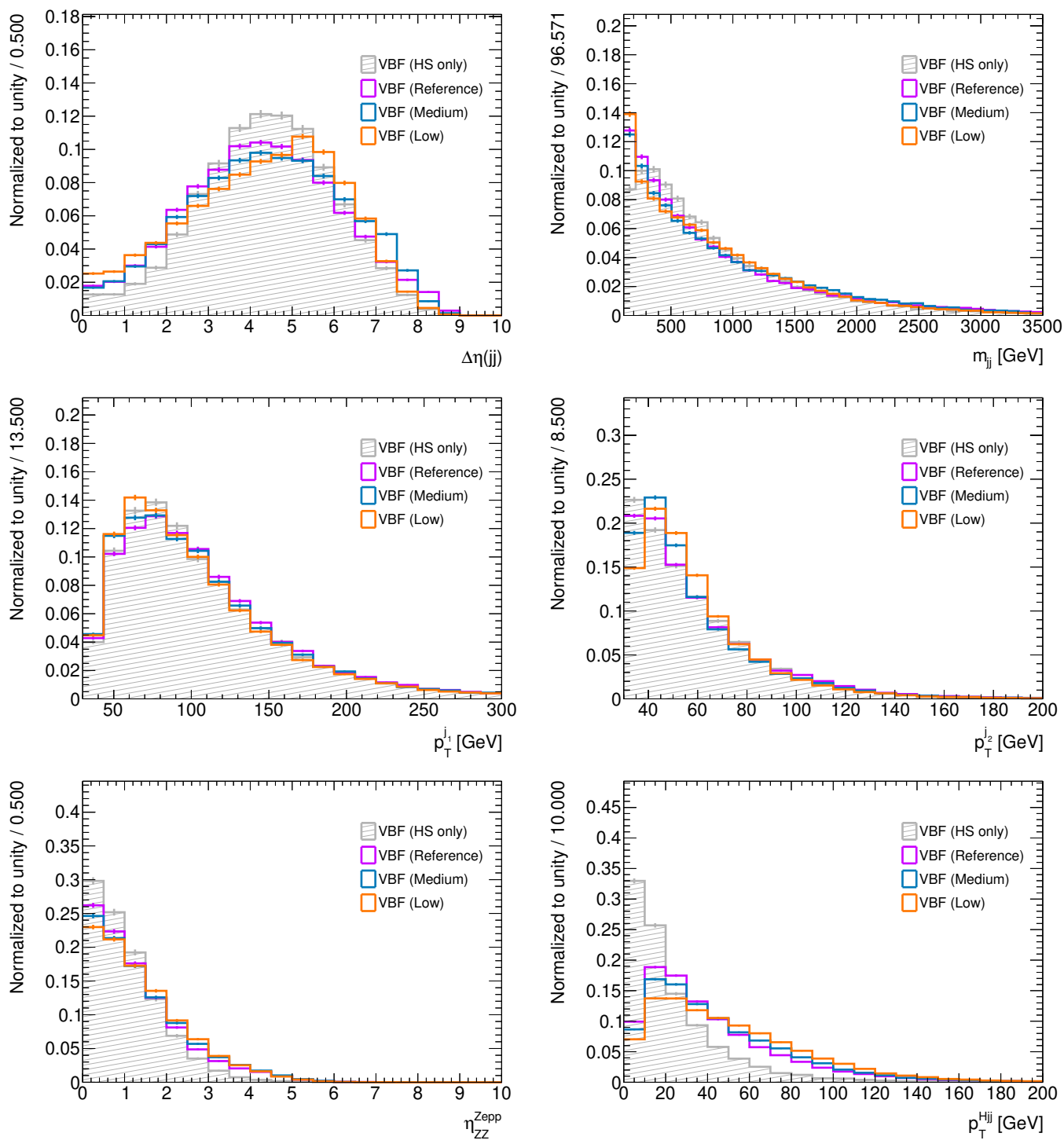

Figure D.1: Comparison of the BDT training variable shapes for VBF $H \rightarrow 4 \ell$ events generated at $\sqrt{s}=14 \mathrm{TeV}$. The events are shown for each upgrade scenario, as well as for the case of no simulated pile-up. 

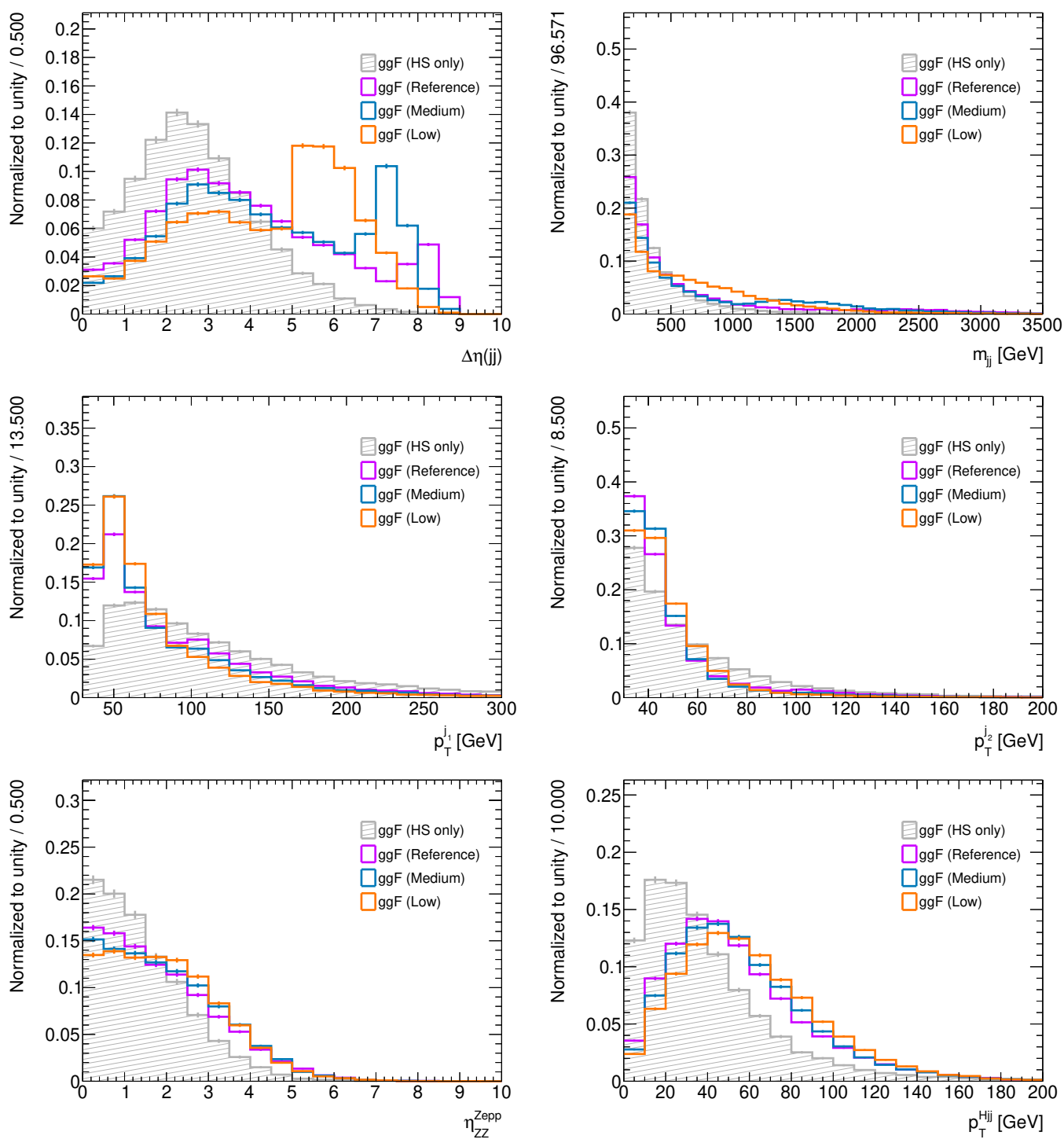

Figure D.2: Comparison of the BDT training variable shapes for ggF $H \rightarrow 4 \ell$ events generated at $\sqrt{s}=14 \mathrm{TeV}$. The events are shown for each upgrade scenario, as well as for the case of no simulated pile-up. 


\begin{tabular}{|c|c|}
\hline \multicolumn{2}{|r|}{ Event pre-selection } \\
\hline Lepton selection & $\begin{array}{l}\text { Require all truth electrons to have } E_{\mathrm{T}}>7 \mathrm{GeV},|\eta|<2.47 \\
\text { Require all truth muons to have } p_{\mathrm{T}}>6 \mathrm{GeV}|\eta|<2.7\end{array}$ \\
\hline \multicolumn{2}{|r|}{ Event selection } \\
\hline $\begin{array}{l}H \rightarrow 4 l \text { kinematic } \\
\text { selection }\end{array}$ & $\begin{array}{l}\text { Require at least one quadruplet of leptons consisting of two pairs of same- } \\
\text { flavour opposite-charge leptons fulfilling the following requirements } \\
p_{\mathrm{T}} \text { thresholds for three leading leptons in the quadruplet } 20,15 \text {, and } 10 \\
\mathrm{GeV} \\
\text { Select best quadruplet to be that with the leading, subleading dilepton } \\
\text { masses closest to the } Z \text { mass } \\
\text { Leading dilepton mass requirement } 50 \mathrm{GeV}<m_{12}<106 \mathrm{GeV} \\
\text { Subleading dilepton mass requirement } 12 \mathrm{GeV}<m_{34}<115 \mathrm{GeV} \\
\text { Remove quadruplet if alternative same-flavour opposite-charge dilepton } \\
\text { gives } m_{l l}<5 \mathrm{GeV} \text { or } \Delta R\left(l, l^{\prime}\right)<0.10(0.20) \text { for same (different) flavour } \\
\text { leptons in the quadruplet }\end{array}$ \\
\hline Dijet selection & $\begin{array}{l}\text { Require } p_{\mathrm{T}}>30 \mathrm{GeV},|\eta|<4.5 \text { for each jet } \\
\text { Remove jets overlapping leptons within } \Delta R<0.2 \\
\text { Dijet mass } m_{\mathrm{jj}}>120 \mathrm{GeV}\end{array}$ \\
\hline
\end{tabular}

Table D.1: Kinematic selection used to isolate truth-level $H \rightarrow 4 l+2 j$ events.

\begin{tabular}{ccc}
\hline \hline Sample & Expected events after $4 l$ selection & Expected events after dijet selection \\
\hline ggF (POWHEG) & 7812 & 621 \\
VBF (POWHEG) & 720 & 358 \\
\hline \hline
\end{tabular}

Table D.2: Expected event counts before and after inclusive and dijet event selection at $\mathcal{I}=$ $3000 \mathrm{fb}^{-1}$ and $\sqrt{s}=14 \mathrm{TeV}$, assuming a Higgs-boson mass of $m_{H}=125.5 \mathrm{GeV}$. 


\section{D.3.2 Definition of the VBF signal region}

The VBF signal region for this analysis was defined by optimizing a pair of cuts on the BDT classifier, thus creating three signal sub-regions: a highly VBF-enriched region; and two regions which add signal significance while maintaining a reasonable signal efficiency. To define the first region, an iterative likelihood scan was performed by tightening the cut on the BDT classifier in an incremental fashion between $0<$ $\mathrm{BDT}<1$. In contrast to the previous $\mathrm{VBF}$ studies presented at $\sqrt{s}=13 \mathrm{TeV}$, the requirement of $\mathrm{BDT}>0$ was necessary limit the presence of pile-up in the signal region. The step size for each iteration was taken to be $\Delta(\mathrm{BDT})=0.1$, as finer step sizes were found to have a minimal impact on the expected results. An integrated luminosity of $\mathcal{I}=3000 \mathrm{fb}^{-1}$ and $\langle\mu\rangle=200$ were assumed. The VBF $H \rightarrow 4 l$ significance $\left(Z_{0}\right)$ was chosen as the metric of improvement for a given set of cuts. At each step of the iteration, the events passing the BDT classifier cut formed the VBF-enriched signal region, such that the cut being that which maximized $Z_{0}$ was taken to be the optimal threshold.

This iterative scan process was repeated to find a second optimal cut to be made between the first cut and BDT $>0$. Accordingly, three signal categories were defined between $0<\mathrm{BDT}<1$, with a final likelihood fit being performed across all three categories simultaneously. For all three detector layout scenarios for $\langle\mu\rangle=200$, sets of categories defined by BDT $\in[0,0.6,0.8,1]$ were found to be the optimal choice in terms of VBF significance. In this construction, the first category defined by $0.8<\mathrm{BDT}<1$ corresponds to the highest purity of VBF signal, and therefore is the strongest contributor to the final significance calculation, while the looser cuts are used to capture as much VBF signal as possible, and miminize the effects of statistical 
uncertainty.

The BDT discriminant distributions for VBF and ggF events are shown in Figure D.3 for each detector layout, given $\langle\mu\rangle=200$ and a pile-up efficiency of $2 \%$. Events with $\geq 1$ pile-up jets induce a large peak around BDT $\sim 0$ in both the VBF and ggF discriminant distributions. Since the BDT was trained on samples containing a mixture of hard scatter and pile-up jets, events with $\geq 1$ pile-up jets would collect in approximately the same score region for both VBF and ggF distributions. However, with increased tracking coverage in $|\eta|$, this feature is both pushed further into the negative score region, and reduces in size. These effects are a consequence of the lower pile-up contamination expected from extended jet tracking; that is to say, with less pile-up contamination, the BDT can use a larger sample of true dijet VBF signal events for training, thereby improving the rejection of both ggF background events, and events with pile-up jets.

\section{D.3.3 $\quad p_{\mathbf{T}}^{H j j}$ and S-T uncertainties for BDT categories}

In this prospects analysis, $p_{\mathrm{T}}^{H j j}$ was used as a training variable in the BDT, therefore the IR-sensitivity effects detailed in Section 7.4 were considered when dividing events into analysis categories based on their BDT score. In particular, before being used in the BDT, the variable was transformed into its "IR-safe" form using Equation 7.6, with the threshold set at $50 \mathrm{GeV}$. Likewise, the Stewart-Tackmann-based uncertainty procedure described in Section 7.4 was employed to evaluate the scale variation uncertainty in each analysis category, with the necessary scale variations being computed at $\sqrt{s}=14 \mathrm{TeV}$ using the parton-level MCFM generator. The resultant S-T uncertainties are given in Table D.3. 


\begin{tabular}{|c|c|}
\hline$p_{\mathrm{T}}^{H j j}$ cut $[\mathrm{GeV}]$ & $\Delta \sigma_{2} / \sigma_{2}(\%)$ \\
\hline 20 & 96.8 \\
\hline 30 & 35.6 \\
\hline 40 & 20.7 \\
\hline 50 & 15.2 \\
\hline 60 & 12.8 \\
\hline 70 & 11.7 \\
\hline 80 & 11.2 \\
\hline 90 & 10.9 \\
\hline
\end{tabular}

Table D.3: Relative S-T uncertainties $\Delta \sigma_{2} / \sigma_{2}$ in bins of $10 \mathrm{GeV}$ rectangular cuts on $p_{\mathrm{T}}^{H j j}$, computed at $\sqrt{s}=14 \mathrm{TeV}$ using the Stewart-Tackmann method.

\section{D.4 Statistical analysis and results}

To assess the prospective VBF signal significance $\left(Z_{0}\right)$ and error on the signal strength $(\Delta \mu / \mu)$ resultant from discriminating dijet VBF and ggF Higgs production, the negative log-likelihood minimization technique was used [131], such that the smeared samples with simulated pile-up were used to perform the negative log-likelihood scans using the VBF $H \rightarrow 4 l$ sample as signal, and the ggF $H \rightarrow 4 l$ sample as background.

The statistical significance of the VBF excess over ggF was computed using the background-only $p$-value $\left(p_{0}\right)$, which is evaluated using the test statistic $q_{\mu}$,

$$
q_{\mu}=-2 \ln \frac{\mathcal{L}(\mu, \theta)}{\mathcal{L}(\hat{\mu}, \hat{\theta})}
$$

such that $q_{0}$ represents the background-only hypothesis, or $\mu=\sigma / \sigma_{\mathrm{SM}}=0$. Here, $\mu$ is the signal strength parameter, while $\theta$ represents the set of nuisance parameters for signal and background. The terms with single circumflexes ( $\hat{\mu}$ and $\hat{\theta}$ ) denote the unconditional maximum likelihood estimates of each parameter. The value of $p_{0}$ is 
defined to be the probability to obtain a value of $q_{0}$ larger than the observed value under the background-only hypothesis. In particular, the value of $p_{0}$ can be expressed as,

$$
p_{0}=\int_{q_{0, \mathrm{obs}}}^{\infty} f\left(q_{0} \mid 0, \hat{\theta}_{0}\right) d q_{0} .
$$

The $p_{0}$ value is then converted to the corresponding number of standard deviations $(\sigma)$ in a one-sided Gaussian test, referred to as the local significance, $Z_{0}$.

The distributions of the BDT discriminant for $\langle\mu\rangle=200$ conditions are shown in Figure D.3, with the corresponding expected VBF significance and signal strength uncertainties given in Table D.4. Overall, it was found that moving from the Reference to Low scenarios led to a degradation of $14 \%$ in $Z_{0}$, and an increase of $\sim 8 \%$ in $\Delta \mu / \mu$. Somewhat surprisingly, the change in VBF significance does not improve as dramatically as one might expect between detector configurations, which in a sense speaks to the robustness of the BDT-based approach in isolating a VBF-like phase space. Regarding the analogous $\langle\mu\rangle=140$ results, it is important to note that this scenario only approximates the expected experimental conditions; while the amount of simulated pile-up jets was reduced to match the expected number of $p p$ bunch crossings, the tracking confirmation used was the same as for the simulations done at $\langle\mu\rangle=200$. Therefore, the estimates given for $\langle\mu\rangle=140$ are likely more pessimistic than one might expect from a more realistic simulation of operating conditions.

The results for these tests are given in Table D.5, both considering only statistical uncertainty, and also Stewart-Tackmann uncertainties. Plots of the corresponding BDT response for each detector layout are shown in Figure D.4. Due to the lower amount of forward pile-up, the performance improvement gained by extended tracking 


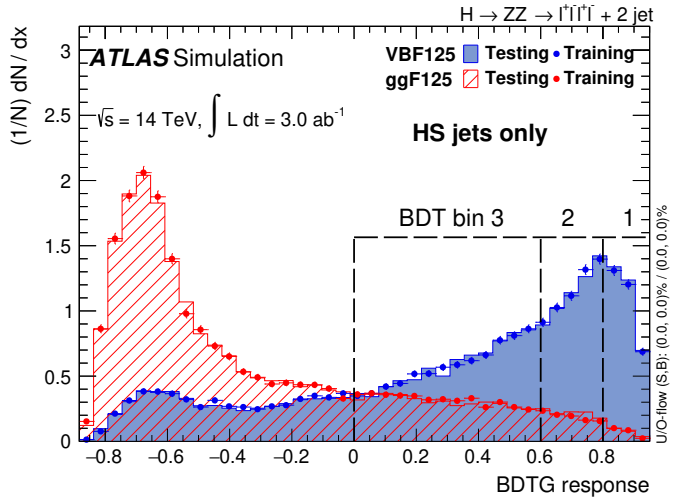

(a) Hard-scatter jets only

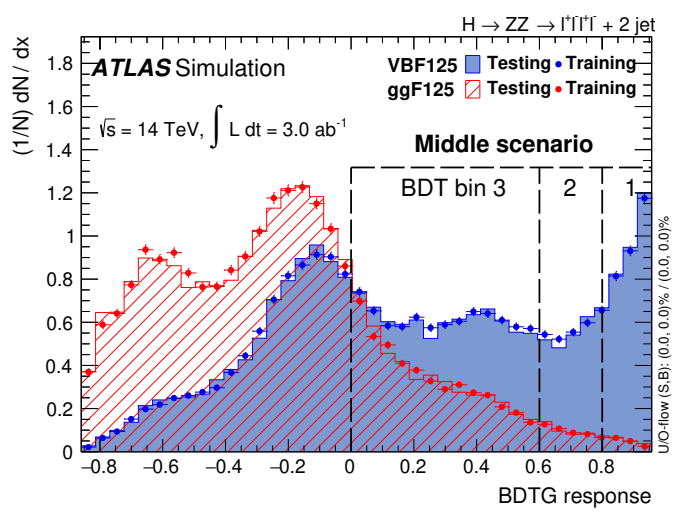

(c) Middle layout

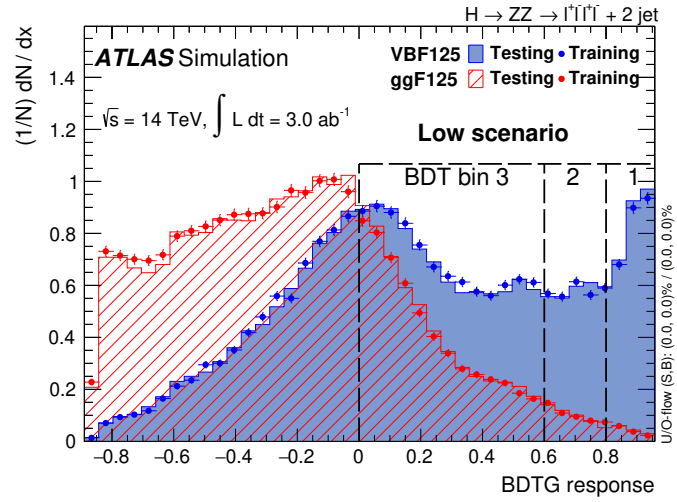

(b) Low layout

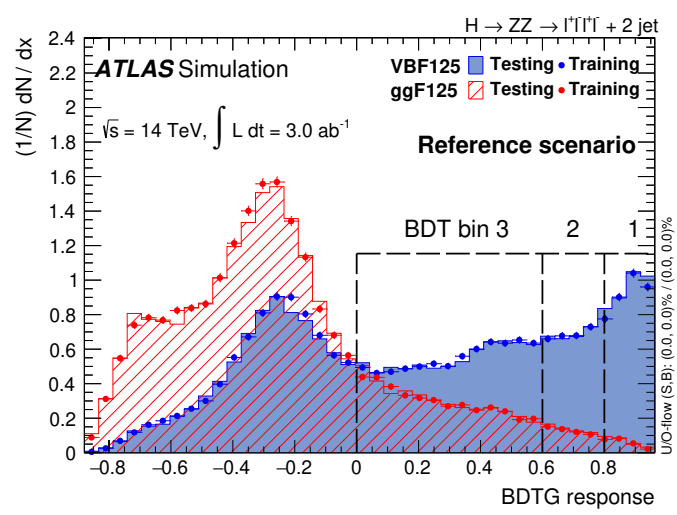

(d) Reference layout

Figure D.3: BDT classifier distributions for Low, Middle, and Reference detector layouts at $\langle\mu\rangle=$ 200 and pile-up efficiency of 0.02 , as well as the response for a BDT trained exclusively on hardscatter jets.

is smaller when considering equivalent tracking scenarios at $\langle\mu\rangle=200$ and 140. It was found that moving from the Reference to Low tracking scenarios for $\langle\mu\rangle=140$ conditions led to a degradation of $8 \%$ in $Z_{0}$, and an increase of $2 \%$ in $\Delta \mu / \mu$. These results are in contrast to the $14 \%$ decrease in $Z_{0}$ and $8 \%$ increase seen in $\Delta \mu / \mu$ for $\langle\mu\rangle=200$. 


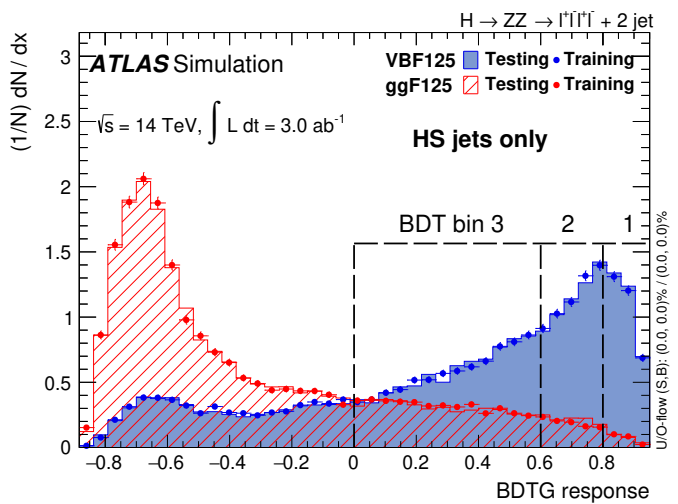

(a) Hard-scatter jets only

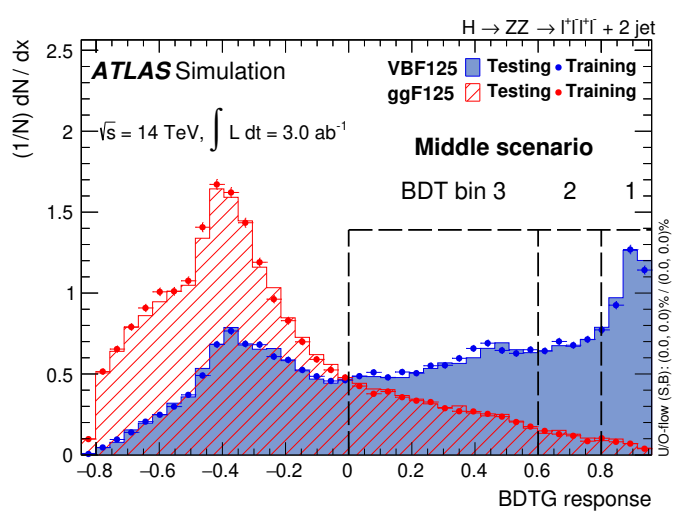

(c) Middle layout

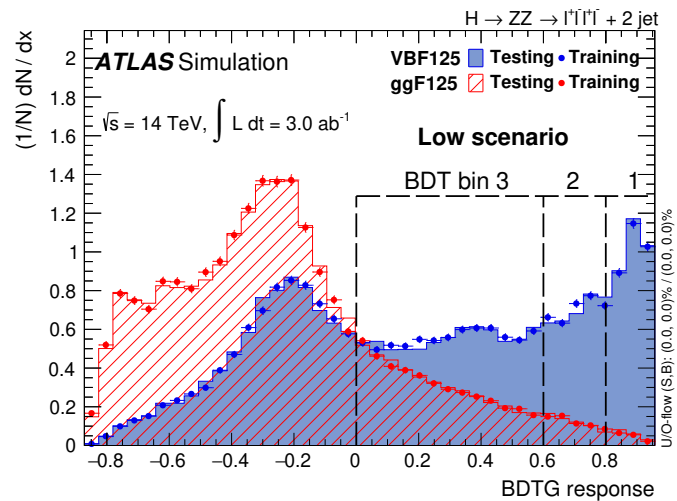

(b) Low layout

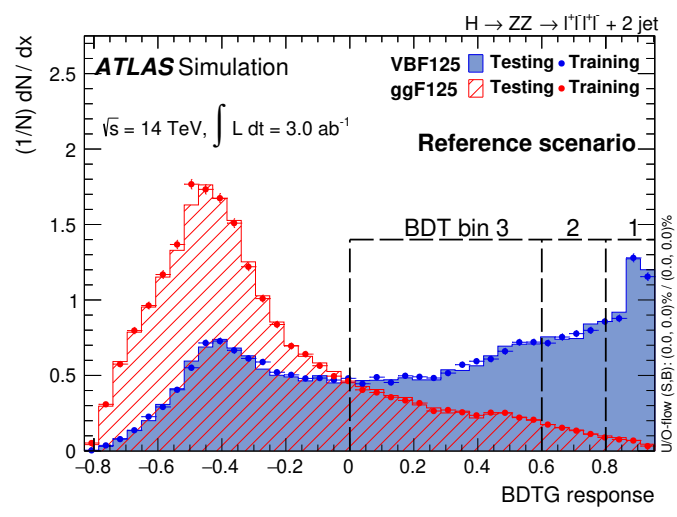

(d) Reference layout

Figure D.4: BDT classifier distributions for Low, Middle, and Reference detector layouts at $\langle\mu\rangle=$ 140 and pile-up efficiency of 0.02 , as well as the response for a BDT trained exclusively on hardscatter jets. 


\begin{tabular}{lccccc}
\hline \hline \multicolumn{7}{c}{ Statistical uncertainty only } \\
\hline Scoping scenario & $\mathrm{VBF}+2 j$ events & $\mathrm{ggF}+2 j$ events & $q q Z Z+2 j$ events & $Z_{0}$ & $\Delta \mu / \mu$ \\
\hline Reference & $192(168)$ & $287(140)$ & $39(16)$ & 10.2 & 0.152 \\
Middle & $218(167)$ & $454(155)$ & $69(15)$ & 9.5 & 0.157 \\
Low & $259(159)$ & $803(182)$ & $124(21)$ & 8.6 & 0.165 \\
\hline \multicolumn{5}{c}{ Statistical } & uncertainty + QCD scale var. uncertainty (S-T method) \\
\hline Scoping scenario & $\mathrm{VBF}+2 j$ events & $\mathrm{ggF}+2 j$ events & $q q Z Z+2 j$ events & $Z_{0}$ & $\Delta \mu / \mu$ \\
\hline Reference & 192 & 287 & 39 & 7.2 & 0.182 \\
Middle & 218 & 454 & 69 & 6.9 & 0.192 \\
Low & 259 & 803 & 124 & 6.2 & 0.208 \\
\hline \hline
\end{tabular}

Table D.4: Expected signal and background event counts for different jet tracking coverage scenarios at $\mathcal{I}=3000 \mathrm{fb}^{-1}$ and $\langle\mu\rangle=200$ in the region (BDT score) $>0$ and $120<m_{4 l}<130 \mathrm{GeV}$, along with the VBF signal significance and signal strength uncertainty for each scenario. Scenarios are shown where only the statistical uncertainty (top), and also Stewart-Tackmann uncertainties (bottom) are considered in the fit. In the first case, the bracketed terms represent the number of events in the signal region with two selected jets from the primary vertex.

\begin{tabular}{lccccc}
\hline \hline \multicolumn{7}{c}{ Statistical uncertainty only } \\
Scoping scenario & VBF $+2 j$ events & ggF $+2 j$ events & $q q Z Z+2 j$ events & $Z_{0}$ & $\Delta \mu / \mu$ \\
\hline Reference & $185(173)$ & $192(121)$ & $23(12)$ & 11.1 & 0.144 \\
Middle & $199(178)$ & $244(126)$ & $30(12)$ & 10.9 & 0.145 \\
Low & $220(178)$ & $385(141)$ & $55(15)$ & 10.2 & 0.148 \\
\hline \hline \multicolumn{5}{c}{ Statistical uncertainty + QCD scale var. uncertainty (S-T method) } \\
\hline Scoping scenario & VBF $+2 j$ events & ggF $+2 j$ events & $q q Z Z+2 j$ events & $Z_{0}$ & $\Delta \mu / \mu$ \\
\hline Reference & 185 & 192 & 23 & 7.7 & 0.170 \\
Middle & 199 & 244 & 30 & 7.7 & 0.173 \\
Low & 220 & 385 & 55 & 7.3 & 0.181 \\
\hline \hline
\end{tabular}

Table D.5: Expected signal and background event counts for different jet tracking coverage scenarios at $\mathcal{I}=3000 \mathrm{fb}^{-1}$ and $\langle\mu\rangle=140$ in the region (BDT score) $>0$ and $120<m_{4 l}<130 \mathrm{GeV}$, along with the VBF signal significance and signal strength uncertainty for each scenario. Scenarios are shown where only the statistical uncertainty (top), and also Stewart-Tackmann uncertainties (bottom) are considered in the fit. In the first case, the bracketed terms represent the number of events in the signal region with two selected jets from the primary vertex. 


\section{Appendix E}

\section{PYTHIA8 settings}

The estimation of uncertainties on the AZNLO parton shower tune in PYTHIA compose differences in:

- Renormalization/factorization scale choice

- Width of the initial parton $p_{\mathrm{T}}$ distributions (or primordial $k_{T}$ )

- The scale of the lower $p_{\mathrm{T}}$ cutoff $p_{\mathrm{T}, 0}^{\mathrm{ref}}$, used to regulate divergences in QCD as $p_{\mathrm{T}} \rightarrow 0$ for the incoming partons

- The scale of the cutoff $p_{\mathrm{T}, 0}^{\mathrm{ref}}$ for space-like showers

The configuration of PyTHIA8 for each parameter variation is given in Table E.1. 


\begin{tabular}{cccccc}
\hline \hline Variation & primordialKThard & SpaceShower:pT0Ref & renormMultFac & factorMultFac & MPI:pT0Ref \\
\hline Nominal & 1.749 & 1.924 & 1 & 1 & 2.002887 \\
Var1 Up & 1.719 & 1.919 & 1 & 1 & 2.002887 \\
Var1 Down & 1.780 & 1.928 & 1 & 1 & 2.002887 \\
Var2 Up & 1.762 & 1.844 & 1 & 1 & 2.002887 \\
Var2 Down & 1.737 & 2.044 & 1 & 1 & 2.002887 \\
FSR Up & 1.749 & 1.924 & 0.5 & 0.5 & 2.002887 \\
FSR Down & 1.749 & 1.924 & 2 & 2 & 2.002887 \\
MPI Up & 1.749 & 1.924 & 1 & 1 & 2.05 \\
MPI Down & 1.749 & 1.924 & 1 & 1 & 1.97 \\
\hline \hline
\end{tabular}

Table E.1: Shower variation settings for the AZNLO tune in Pythia8. 


\section{References}

[1] ATLAS Collaboration, Observation of a new particle in the search for the Standard Model Higgs boson with the ATLAS detector at the LHC, Phys. Lett. B716 (2012) 1-29, arXiv:1207.7214 [hep-ex].

[2] CMS Collaboration, Observation of a new boson at a mass of $125 \mathrm{GeV}$ with the CMS experiment at the LHC, Phys. Lett. B716 (2012) 30-61, arXiv:1207.7235 [hep-ex].

[3] ATLAS Collaboration, Study of the spin and parity of the Higgs boson in diboson decays with the ATLAS detector, Eur. Phys. J. C75 (2015) no. 10, 476, arXiv:1506.05669 [hep-ex]. [Erratum: Eur. Phys.

J.C76,no.3,152(2016)].

[4] ATLAS Collaboration, Test of CP Invariance in vector-boson fusion production of the Higgs boson using the Optimal Observable method in the ditau decay channel with the ATLAS detector, Eur. Phys. J. C76 (2016) no. 12,658 , arXiv:1602.04516 [hep-ex]

[5] CMS Collaboration, Study of the Mass and Spin-Parity of the Higgs Boson Candidate Via Its Decays to Z Boson Pairs, Phys. Rev. Lett. 110 (2013) 
no. 8,081803, arXiv:1212.6639 [hep-ex].

[6] CMS Collaboration, Constraints on the spin-parity and anomalous HVV couplings of the Higgs boson in proton collisions at 7 and $8 \mathrm{TeV}$, Phys. Rev. D92 (2015) no. 1, 012004, arXiv:1411.3441 [hep-ex].

[7] M. Peskin and D. Schroeder, An Introduction To Quantum Field Theory (Frontiers in Physics). Westview Press, 1995.

[8] P. W. Higgs, Broken symmetries and the masses of gauge bosons, Phys. Rev. Lett. 13 (1964) 508-509.

[9] F. Englert and R. Brout, Broken symmetry and the mass of gauge vector mesons, Phys. Rev. Lett. 13 (1964) 321-323.

[10] G. Guralnik, C. Hagen, and T. Kibble, Global conservation laws and massless particles, Phys. Rev. Lett. 13 (1964) 585-587.

[11] Y. Nambu, Quasi-Particles and Gauge Invariance in the Theory of Superconductivity, Phys. Rev. 117 (1960) 648-663. https://link.aps.org/doi/10.1103/PhysRev.117.648.

[12] P. W. Higgs, Spontaneous Symmetry Breakdown without Massless Bosons, Phys. Rev. 145 (1966) 1156-1163. https://link.aps.org/doi/10.1103/PhysRev.145.1156.

[13] S. Nussinov and R. Shrock, Upper Limits on a Possible Gluon Mass, Phys. Rev. D82 (2010) 034031, arXiv:1005.0850 [hep-ph]. 
[14] SNO Collaboration, Electron energy spectra, fluxes, and day-night asymmetries of B-8 solar neutrinos from measurements with $\mathrm{NaCl}$ dissolved in the heavy-water detector at the Sudbury Neutrino Observatory, Phys. Rev. C72 (2005) 055502, arXiv:nucl-ex/0502021 [nucl-ex].

[15] Super-Kamiokande Collaboration, Evidence for oscillation of atmospheric neutrinos, Phys. Rev. Lett. 81 (1998) 1562-1567, arXiv:hep-ex/9807003 [hep-ex] .

[16] KamLAND-Zen Collaboration, A. Gando et al., Search for Majorana Neutrinos near the Inverted Mass Hierarchy Region with KamLAND-Zen, Phys. Rev. Lett. 117 (2016) no. 8, 082503, arXiv:1605.02889 [hep-ex]. [Addendum: Phys. Rev. Lett.117,no.10,109903(2016)].

[17] GERDA Collaboration, The search for $0 \nu \beta \beta$ decay with the GERDA experiment: Status and prospects, AIP Conf. Proc. 1672 (2015) 110003, arXiv:1506.00415 [hep-ex].

[18] EXO-200 Collaboration, Search for Neutrinoless Double-Beta Decay in ${ }^{136}$ Xe with EXO-200, Phys. Rev. Lett. 109 (2012) 032505, arXiv:1205.5608 [hep-ex] .

[19] J. C. Collins, D. E. Soper, and G. F. Sterman, Factorization of Hard Processes in $Q C D$, Adv. Ser. Direct. High Energy Phys. 5 (1989) 1-91, arXiv:hep-ph/0409313 [hep-ph].

[20] ATLAS Collaboration, Performance of b-Jet Identification in the ATLAS Experiment, JINST 11 (2016) no. 04, P04008, arXiv:1512.01094 [hep-ex]. 
[21] J. Gao, M. Guzzi, J. Huston, H.-L. Lai, Z. Li, P. Nadolsky, J. Pumplin, D. Stump, and C. P. Yuan, CT10 next-to-next-to-leading order global analysis of QCD, Phys. Rev. D89 (2014) no. 3, 033009, arXiv:1302.6246 [hep-ph].

[22] A. Djouadi, The anatomy of electroweak symmetry breaking: Tome I: The Higgs boson in the Standard Model, Physics Reports 457 (2008) no. 1 - 4, 1 216.

[23] CMS Collaboration, W. de Boer, The Discovery of the Higgs Boson with the CMS Detector and its Implications for Supersymmetry and Cosmology, in Time and Matter 2013 (TAM2013) Venice, Italy. 2013. arXiv:1309.0721 [hep-ph] .

[24] SLAC E158 Collaboration, M. Woods, A New measurement of the weak mixing angle, eConf C0307282 (2003) TTH04, arXiv:hep-ex/0403010 [hep-ex].

[25] CMS Collaboration, Measurement of the weak mixing angle with the Drell-Yan process in proton-proton collisions at the LHC, Phys. Rev. D84 (2011) 112002, arXiv:1110.2682 [hep-ex].

[26] ATLAS Collaboration, Measurement of the forward-backward asymmetry of electron and muon pair-production in pp collisions at $\sqrt{s}=7 \mathrm{TeV}$ with the ATLAS detector, JHEP 09 (2015) 049, arXiv:1503.03709 [hep-ex].

[27] CDF, D0 Collaborations, Combination of CDF and D0 W-Boson Mass Measurements, Phys. Rev. D88 (2013) no. 5, 052018, arXiv:1307.7627 [hep-ex] . 
[28] ATLAS Collaboration, ATLAS Collaboration, Measurement of the $W$-boson mass in pp collisions at $\sqrt{s}=7 \mathrm{TeV}$ with the ATLAS detector, Eur. Phys. J. C78 (2018) no. 2, 110, arXiv:1701.07240 [hep-ex].

[29] Line Shape Sub-Group of the LEP Electroweak Working Group, DELPHI, LEP, ALEPH, OPAL, L3 Collaborations, Combination procedure for the precise determination of $Z$ boson parameters from results of the LEP experiments, arXiv:hep-ex/0101027 [hep-ex].

[30] G. C. Wick, The Evaluation of the Collision Matrix, Phys. Rev. 80 (1950) . https://link.aps.org/doi/10.1103/PhysRev.80.268.

[31] LHC Higgs Cross Section Working Group Collaboration, Handbook of LHC Higgs Cross Sections: 4. Deciphering the Nature of the Higgs Sector, arXiv:1610.07922 [hep-ph].

[32] C. Anastasiou, R. Boughezal, and F. Petriello, Mixed QCD-electroweak corrections to Higgs boson production in gluon fusion, JHEP 04 (2009) 003, arXiv:0811.3458 [hep-ph].

[33] T. Han, G. Valencia, and S. Willenbrock, Structure function approach to vector boson scattering in pp collisions, Phys. Rev. Lett. 69 (1992) 3274-3277.

[34] P. Bolzoni, F. Maltoni, S. Moch, and M. Zaro, Higgs production via vector-boson fusion at NNLO in QCD, Phys. Rev. Lett. 105 (2010) 011801, arXiv:1003.4451 [hep-ph].

[35] J. M. Campbell, R. K. Ellis, and C. Williams, Associated production of a Higgs boson at NNLO, JHEP 06 (2016) 179, arXiv:1601.00658 [hep-ph]. 
[36] M. Ciccolini, A. Denner, and S. Dittmaier, Strong and electroweak corrections to the production of Higgs + 2jets via weak interactions at the LHC, Phys. Rev. Lett. 99 (2007) 161803, arXiv:0707.0381 [hep-ph].

[37] M. L. Ciccolini, S. Dittmaier, and M. Kramer, Electroweak radiative corrections to associated WH and ZH production at hadron colliders, Phys. Rev. D68 (2003) 073003, arXiv:hep-ph/0306234 [hep-ph].

[38] J. Ellis, M. K. Gaillard, and D. Nanopoulos, A phenomenological profile of the Higgs boson, Nuclear Physics B 106 (1976) 292 - 340. http:

//www.sciencedirect.com/science/article/pii/0550321376903825.

[39] Particle Data Group Collaboration, C. Patrignani et al., Review of Particle Physics, Chin. Phys. C40 (2016) no. 10, 100001.

[40] A. Bredenstein, A. Denner, S. Dittmaier, and M. M. Weber, Precise predictions for the Higgs-boson decay $H-i W W / Z Z-i$ 4 leptons, Phys. Rev. D74 (2006) 013004, arXiv:hep-ph/0604011 [hep-ph].

[41] R. Tanaka, C. Mariotti, S. Heinemeyer, and G. Passarino, LHC Higgs Cross Section Working Group, https://twiki.cern.ch/twiki/bin/view/LHCPhysics/CrossSections, All figures used with permission of LHC HXSWG, April, 2017.

[42] K. G. Wilson, The Renormalization Group and Strong Interactions, Phys. Rev. D3 (1971) 1818.

[43] J. F. Gunion, Extended Higgs sectors, in Supersymmetry and unification of fundamental interactions. Proceedings, 10th International Conference, 
SUSY'02, Hamburg, Germany, June 17-23, 2002, pp. 80-103. 2002. arXiv:hep-ph/0212150 [hep-ph].

[44] J. Dietrich, Overview of the SUSY results from the ATLAS and CMS experiments, . http://cds.cern.ch/record/1642786.

[45] M. Perelstein, Little Higgs models and their phenomenology, Prog. Part. Nucl. Phys. 58 (2007) 247-291, arXiv:hep-ph/0512128 [hep-ph] .

[46] Planck Collaboration, Planck 2013 results. I. Overview of products and scientific results, Astron. Astrophys. 571 (2014) A1, arXiv:1303.5062 [astro-ph.CO].

[47] H. Goldberg, Constraint on the Photino Mass from Cosmology, Phys. Rev. Lett. 50 (1983) 1419.

[48] J. R. Ellis, J. S. Hagelin, D. V. Nanopoulos, K. A. Olive, and M. Srednicki, Supersymmetric Relics from the Big Bang, Nucl. Phys. B238 (1984) 453-476.

[49] C. Munoz, Models of Supersymmetry for Dark Matter, arXiv:1701.05259 [hep-ph]. [EPJ Web Conf.13,60100(2017)].

[50] ATLAS, CMS Collaboration, A. de Cosa, LHC results for dark matter from ATLAS and CMS, in Proceedings, 12th Conference on the Intersections of Particle and Nuclear Physics (CIPANP 2015): Vail, Colorado, USA, May 19-24, 2015. 2015. arXiv:1510.01516 [hep-ex].

[51] ATLAS Collaboration, Evidence for the spin-0 nature of the Higgs boson using ATLAS data, Phys. Lett. B726 (2013) 120-144, arXiv:1307.1432 [hep-ex]. 
[52] ATLAS Collaboration, Measurement of inclusive and differential cross sections in the $H \rightarrow Z Z^{*} \rightarrow 4 \ell$ decay channel in pp collisions at $\sqrt{s}=13 \mathrm{TeV}$ with the ATLAS detector, arXiv:1708.02810 [hep-ex].

[53] ATLAS Collaboration, Combined measurements of Higgs boson production and decay in the $H \rightarrow Z Z \rightarrow 4 \ell$ and $H \rightarrow \gamma \gamma$ channels using $\sqrt{s}=13 \mathrm{TeV} p p$ collision data collected with the ATLAS experiment, ATLAS-CONF-2017-047, CERN, Geneva, Jul, 2017. http://cds. cern.ch/record/2273854.

[54] L. Evans and P. Bryant, LHC Machine, JINST 3 (2008) S08001.

[55] ATLAS Collaboration, The ATLAS Experiment at the CERN Large Hadron Collider, Journal of Instrumentation 3 (2008) no. 08, S08003.

[56] CMS Collaboration, The CMS Experiment at the CERN LHC, JINST 3 (2008) S08004.

[57] ALICE Collaboration, The ALICE experiment at the CERN LHC, JINST 3 (2008) S08002.

[58] LHCb Collaboration, A. A. Alves, Jr. et al., The LHCb Detector at the LHC, JINST 3 (2008) S08005.

[59] W. Herr and B. Muratori, Concept of luminosity, . http://cds.cern.ch/record/941318.

[60] CDF Collaboration, Charged jet evolution and the underlying event in $p \bar{p}$ collisions at 1.8 TeV, Phys. Rev. D65 (2002) 092002. 
[61] G. G. Barnafoldi, A. G. Agocs, and P. Levai, Underlying Event Studies for LHC Energies, AIP Conf. Proc. 1348 (2011) 124-129, arXiv:1101.4155 [hep-ph] .

[62] ATLAS Collaboration, Track Reconstruction Performance of the ATLAS Inner Detector at $\sqrt{s}=13 \mathrm{TeV}$, ATL-PHYS-PUB-2015-018, 2015. https://cds. cern. ch/record/2037683.

[63] ATLAS Collaboration, F. Hügging, The ATLAS Pixel Insertable B-layer (IBL), Nuclear Instruments and Methods in Physics Research A (2011), arXiv:1012.2742 [physics.ins-det].

[64] ATLAS Collaboration, ATLAS detector and physics performance: Technical Design Report. CERN, Geneva, 1999.

[65] J. Pequenao, Computer Generated image of the ATLAS calorimeter, Mar, 2008.

[66] ATLAS Collaboration, Performance of the ATLAS Trigger System in 2010, Eur. Phys. J. C72 (2012) 1849, arXiv:1110.1530 [hep-ex].

[67] ATLAS Collaboration, Performance of the ATLAS Trigger System in 2010, Eur. Phys. J. C 72 (2012) 1849, arXiv:1110.1530 [hep-ex].

[68] W. Lampl et al., Calorimeter Clustering Algorithms: Description and Performance, ATL-LARG-PUB-2008-002, 2008. https://cds. cern.ch/record/1099735. 
[69] T. G. Cornelissen, M. Elsing, I. Gavrilenko, J. F. Laporte, W. Liebig, M. Limper, K. Nikolopoulos, A. Poppleton, and A. Salzburger, The global $\chi^{2}$ track fitter in ATLAS, J. Phys. Conf. Ser. 119 (2008) 032013.

[70] R. Fruhwirth, Application of Kalman filtering to track and vertex fitting, Nucl. Instrum. Meth. A262 (1987) 444-450.

[71] ATLAS Collaboration, Electron efficiency measurements with the ATLAS detector using 2012 LHC proton-proton collision data, arXiv:1612.01456 [hep-ex].

[72] ATLAS Collaboration, Measurement of the photon identification efficiencies with the ATLAS detector using LHC Run-1 data, arXiv:1606.01813 [hep-ex].

[73] ATLAS Collaboration, Electron and photon energy calibration with the ATLAS detector using data collected in 2015 at $\sqrt{s}=13 \mathrm{TeV}$, ATL-PHYS-PUB-2016-015, 2016. https://cds . cern. ch/record/2203514.

[74] ATLAS Collaboration, Topological cell clustering in the ATLAS calorimeters and its performance in LHC Run 1, arXiv:1603.02934 [hep-ex].

[75] NA48 Collaboration, Performance of an electromagnetic liquid krypton calorimeter, Nucl. Instrum. Meth. A344 (1994) 507-520.

[76] ATLAS Collaboration, Electron performance of the ATLAS detector using the $J / \psi \rightarrow e^{+} e^{-}$decays, ATL-COM-PHYS-2010-518. 
[77] ATLAS Collaboration, Performance of the ATLAS electromagnetic calorimeter for $\pi^{0} \rightarrow \gamma \gamma$ and $\eta \rightarrow \gamma \gamma$ events, ATLAS-CONF-2010-006, 2010. https://cds. cern.ch/record/1273999.

[78] GEANT4 Collaboration, S. Agostinelli et al., GEANT4: A Simulation toolkit, Nucl. Instrum. Meth. in Phys. Res. A506 (2003) 250-303.

[79] J. Allison, K. Amako, J. Apostolakis, H. Araujo, P. Dubois, et al., GEANT4 developments and applications, IEEE Trans. Nucl. Sci. 53 (2006) 270-278.

[80] ATLAS Collaboration, Electron identification measurements in ATLAS using $\sqrt{s}=13$ TeV data with 50 ns bunch spacing, . https://cds.cern.ch/record/2048202.

[81] ATLAS Collaboration, Photon identification in 2015 ATLAS data, ATL-PHYS-PUB-2016-014, 2016. https://cds . cern. ch/record/2203125.

[82] A. Hoecker, P. Speckmayer, J. Stelzer, J. Therhaag, E. von Toerne, H. Voss, et al., TMVA: Toolkit for Multivariate Data Analysis, Proceedings of Science ACAT (2007) 040, arXiv:physics/0703039.

[83] ATLAS Collaboration, Electron and photon energy calibration with the ATLAS detector using LHC Run 1 data, arXiv:1407.5063 [hep-ex].

[84] C. Oleari, The POWHEG-BOX, Nucl. Phys. Proc. Suppl. 205-206 (2010) 36-41, arXiv:1007.3893 [hep-ph].

[85] T. Sjostrand, S. Mrenna, and P. Z. Skands, A Brief Introduction to PYTHIA 8.1, Comput. Phys. Commun. 178 (2008) 852-867, arXiv:0710.3820 [hep-ph]. 
[86] ATLAS Collaboration, Measurement of the Higgs boson coupling properties in the $H \rightarrow Z Z^{*} \rightarrow 4 \ell$ decay channel at $\sqrt{s}=13 \mathrm{TeV}$ with the ATLAS detector, ATLAS-CONF-2017-043, CERN, Geneva, Jul, 2017.

http://cds. cern. ch/record/2273849.

[87] S. Weinzierl, Introduction to Monte Carlo methods, arXiv:hep-ph/0006269 [hep-ph] .

[88] F. Hautmann, Heavy top limit and double logarithmic contributions to Higgs production at $m_{H}^{2} / s<<1$, Phys. Lett. B535 (2002) 159-162, arXiv:hep-ph/0203140 [hep-ph].

[89] K. Hamilton, P. Nason, E. Re, and G. Zanderighi, NNLOPS simulation of Higgs boson production, JHEP 10 (2013) 222, arXiv:1309.0017 [hep-ph].

[90] K. Hamilton, P. Nason, and G. Zanderighi, MINLO: Multi-Scale Improved NLO, JHEP 10 (2012) 155, arXiv:1206.3572 [hep-ph].

[91] S. Catani and M. Grazzini, HNNLO: A Monte Carlo program to compute Higgs boson production at hadron colliders, arXiv:0802.1410 [hep-ph].

[92] J. Alwall, R. Frederix, S. Frixione, V. Hirschi, F. Maltoni, O. Mattelaer, H. S. Shao, T. Stelzer, P. Torrielli, and M. Zaro, The automated computation of tree-level and next-to-leading order differential cross sections, and their matching to parton shower simulations, JHEP 07 (2014) 079, arXiv:1405.0301 [hep-ph]. 
[93] K. Hamilton, P. Nason, and G. Zanderighi, Finite quark-mass effects in the NNLOPS POWHEG+MiNLO Higgs generator, JHEP 05 (2015) 140, arXiv:1501.04637 [hep-ph].

[94] M. Grazzini and H. Sargsyan, Heavy-quark mass effects in Higgs boson production at the LHC, JHEP 09 (2013) 129, arXiv:1306.4581 [hep-ph].

[95] S. Gangal and F. J. Tackmann, Next-to-leading-order uncertainties in Higgs+2 jets from gluon fusion, Phys. Rev. D87 (2013) no. 9, 093008, arXiv:1302.5437 [hep-ph].

[96] F. Demartin, E. Vryonidou, K. Mawatari, and M. Zaro, Higgs characterisation: $N L O$ and parton-shower effects, in 2nd Toyama International Workshop on Higgs as a Probe of New Physics (HPNP2015) Toyama, Japan, February 11-15, 2015. 2015. arXiv:1505.07081 [hep-ph].

[97] F. Maltoni, K. Mawatari, and M. Zaro, Higgs characterisation via vector-boson fusion and associated production: NLO and parton-shower effects, Eur. Phys. J. C74 (2014) no. 1, 2710, arXiv:1311.1829 [hep-ph].

[98] ATLAS Collaboration, A morphing technique for signal modelling in a multidimensional space of coupling parameters, ATL-PHYS-PUB-2015-047, 2015. https://cds. cern. ch/record/2066980.

[99] A. Kaluza, Development of morphing for studies of HEFT with ATLAS, Higgs Effective Field Theories workshop, November, 2015.

[100] N. Belyaev, Monte Carlo for the tensor structure analysis in the Higgs sector, Monte Carlo reweighting workshop, May, 2015. 
[101] J. Butterworth et al., PDF 4 LHC recommendations for LHC Run II, J. Phys. G43 (2016) 023001, arXiv:1510.03865 [hep-ph].

[102] P. Nason and C. Oleari, NLO Higgs boson production via vector-boson fusion matched with shower in POWHEG, JHEP 02 (2010) 037, arXiv:0911.5299 [hep-ph].

[103] G. Luisoni, P. Nason, C. Oleari, and F. Tramontano, $H W^{ \pm} / H Z+0$ and 1 jet at NLO with the POWHEG BOX interfaced to GoSam and their merging within MiNLO, JHEP 10 (2013) 083, arXiv:1306.2542 [hep-ph].

[104] ATLAS Collaboration, G. Aad et al., Measurement of the $Z / \gamma^{*}$ boson transverse momentum distribution in pp collisions at $\sqrt{s}=7 \mathrm{TeV}$ with the ATLAS detector, JHEP 09 (2014) 145, arXiv:1406.3660 [hep-ex].

[105] M. Bahr et al., Herwig++ Physics and Manual, Eur. Phys. J. C58 (2008) 639-707, arXiv:0803.0883 [hep-ph].

[106] ATLAS Collaboration, Muon reconstruction performance of the ATLAS detector in proton-proton collision data at $\sqrt{s}=13 \mathrm{TeV}$, Eur. Phys. J. C76 (2016) no. 5, 292, arXiv:1603.05598 [hep-ex].

[107] ATLAS Collaboration, Measurement of the muon reconstruction performance of the ATLAS detector using 2011 and 2012 LHC protonproton collision data, Eur. Phys. J. C74 (2014) no. 11, 3130, arXiv:1407.3935 [hep-ex].

[108] G. Cree, Direct measurement of the Higgs boson mass, natural width, and cross section times branching ratio to four leptons using a per-event lineshape 
in the Higgs to ZZ to four lepton decay channel with the ATLAS detector. $\mathrm{PhD}$ thesis, Carleton University, 2017.

[109] M. Cacciari and G. P. Salam, Dispelling the $N^{3}$ myth for the $k_{t}$ jet-finder, Phys. Lett. B 641 (2006) 57, arXiv:hep-ph/0512210.

[110] M. Cacciari, G. P. Salam, and G. Soyez, The Anti-k(t) jet clustering algorithm, JHEP 04 (2008) 063, arXiv:0802.1189 [hep-ph].

[111] ATLAS Collaboration, Jet energy scale measurements and their systematic uncertainties in proton-proton collisions at $\sqrt{s}=13 \mathrm{TeV}$ with the ATLAS detector, arXiv:1703.09665 [hep-ex].

[112] ATLAS Collaboration, Jet calibration and systematic uncertainties for jets reconstructed in the ATLAS detector at $\sqrt{s}=13 \mathrm{TeV}$, Atl-phys-pub-2015-015. http://cdsweb. cern. ch/record/2037613.

[113] M. Cacciari and G. P. Salam, Pileup subtraction using jet areas, Phys. Lett. B659 (2008) 119-126, arXiv:0707.1378 [hep-ph].

[114] ATLAS Collaboration, Selection of jets produced in 13 TeV proton-proton collisions with the ATLAS detector, ATLAS-CONF-2015-029, 2015. https://cds.cern.ch/record/2037702.

[115] ATLAS Collaboration, Tagging and suppression of pileup jets with the ATLAS detector, ATLAS-CONF-2014-018, 2014. https://cds.cern.ch/record/1700870.

[116] ATLAS Collaboration, Performance of b-Jet Identification in the ATLAS Experiment, JINST 11 (2016) P04008, arXiv:1512.01094 [hep-ex]. 
[117] T. Gleisberg, S. Hoeche, F. Krauss, M. Schonherr, S. Schumann, et al., Event generation with SHERPA 1.1, JHEP 0902 (2009) 007, arXiv:0811.4622 [hep-ph] .

[118] T. Gleisberg and S. Höche, Comix, a new matrix element generator, JHEP 0812 (2008) 039, arXiv:0808.3674 [hep-ph].

[119] F. Cascioli, P. Maierhofer, and S. Pozzorini, Scattering Amplitudes with Open Loops, Phys. Rev. Lett. 108 (2012) 111601, arXiv:1111.5206 [hep-ph].

[120] S. Schumann and F. Krauss, A Parton shower algorithm based on Catani-Seymour dipole factorisation, JHEP 0803 (2008) 038, arXiv:0709.1027 [hep-ph].

[121] S. Höche, F. Krauss, M. Schönherr, and F. Siegert, QCD matrix elements + parton showers: The NLO case, JHEP 04 (2013) 027, arXiv:1207.5030 [hep-ph].

[122] B. Biedermann, A. Denner, S. Dittmaier, L. Hofer, and B. Jger, Electroweak corrections to $p p \rightarrow \mu^{+} \mu^{-} e^{+} e^{-}+X$ at the LHC: a Higgs background study, Phys. Rev. Lett. 116 (2016) no. 16, 161803, arXiv:1601.07787 [hep-ph].

[123] B. Biedermann, A. Denner, S. Dittmaier, L. Hofer, and B. Jager, Next-to-leading-order electroweak corrections to the production of four charged leptons at the LHC, JHEP 01 (2017) 033, arXiv:1611.05338 [hep-ph] .

[124] N. Kauer, C. OBrien, and E. Vryonidou, Interference effects for $H \rightarrow W W \rightarrow \ell \nu q \bar{q}^{\prime}$ and $H \rightarrow Z Z \rightarrow \ell \bar{\ell} q \bar{q}$ searches in gluon fusion at the LHC, JHEP 10 (2015) 074, arXiv:1506.01694 [hep-ph] . 
[125] F. Caola, K. Melnikov, R. Rntsch, and L. Tancredi, QCD Corrections to ZZ Production in Gluon Fusion at the LHC, Phys. Rev. D92 (2015) no. 9, 094028, arXiv:1509.06734 [hep-ph].

[126] J. M. Campbell, R. K. Ellis, M. Czakon, and S. Kirchner, Two loop correction to interference in $g g \rightarrow Z Z$, JHEP 08 (2016) 011, arXiv: 1605.01380 [hep-ph] .

[127] C. S. Li, H. T. Li, D. Y. Shao, and J. Wang, Soft gluon resummation in the signal-background interference process of $g g\left(\rightarrow h^{*}\right) \rightarrow Z Z$, JHEP 08 (2015) 065, arXiv: 1504.02388 [hep-ph].

[128] ATLAS Collaboration, Measurement of Higgs boson production in the diphoton decay channel in pp collisions at center-of-mass energies of 7 and 8 TeV with the ATLAS detector, Phys. Rev. D90 (2014) no. 11, 112015, arXiv:1408.7084 [hep-ex].

[129] ATLAS Collaboration, Measurements of Higgs boson production and couplings in the four-lepton channel in pp collisions at center-of-mass energies of 7 and 8 TeV with the ATLAS detector, Phys. Rev. D91 (2015) no. 1, 012006, arXiv:1408.5191 [hep-ex].

[130] J. Friedman, Stochastic gradient boosting, Computational Statistics \& Data Analysis 38 (2002) no. 4, $367-378$.

[131] G. Cowan, K. Cranmer, E. Gross, and O. Vitells, Asymptotic formulae for likelihood-based tests of new physics, Eur. Phys. J. C71 (2011) 1554, 1-19, arXiv:1007.1727 [physics.data-an]. 
[132] S. Gangal, Precision Predictions of Exclusive Jet Cross Sections at the LHC. PhD thesis, U. Hamburg, Dept. Phys., Hamburg, 2015. http://bib-pubdb1. desy $\cdot$ de/search?cc=Publication+Database\&of=hd\&p= reportnumber:DESY-THESIS-2015-042.

[133] J. M. Campbell and R. Ellis, MCFM for the Tevatron and the LHC, Nucl. Phys. Proc. Suppl. 205-206 (2010) 10-15, arXiv:1007.3492 [hep-ph].

[134] I. W. Stewart and F. J. Tackmann, Theory Uncertainties for Higgs and Other Searches Using Jet Bins, Phys. Rev. D85 (2012) 034011, arXiv:1107.2117 [hep-ph] .

[135] ROOT Collaboration, K. Cranmer, G. Lewis, L. Moneta, A. Shibata, and W. Verkerke, HistFactory: A tool for creating statistical models for use with RooFit and RooStats, CERN-OPEN-2012-016, New York U., New York, Jan, 2012. https://cds. cern. ch/record/1456844.

[136] L. Moneta, K. Cranmer, G. Schott, and W. Verkerke, The RooStats project, in Proceedings of the 13th International Workshop on Advanced Computing and Analysis Techniques in Physics Research. 2010. arXiv:1009.1003.

[137] ATLAS Collaboration, Luminosity determination in pp collisions at $\sqrt{s}=8 \mathrm{TeV}$ using the ATLAS detector at the LHC, Eur. Phys. J. C 76 (2016) 653, arXiv:1608.03953 [hep-ex].

[138] ATLAS Collaboration, Measurement of the Inelastic Proton-Proton Cross Section at $\sqrt{s}=13 \mathrm{TeV}$ with the ATLAS Detector at the LHC, Phys. Rev. Lett. 117 (2016) 182002, arXiv:1606.02625 [hep-ex]. 
[139] ATLAS Collaboration, Electron efficiency measurements with the ATLAS detector using the 2012 LHC proton-proton collision data, ATLAS-CONF-2014-032, 2014. https://cds.cern.ch/record/1706245.

[140] ATLAS Collaboration, Muon reconstruction performance of the ATLAS detector in proton-proton collision data at $\sqrt{s}=13 \mathrm{TeV}$, Eur. Phys. J. C 76 (2016) 292, arXiv:1603.05598 [hep-ex] .

[141] ATLAS Collaboration, Electron and photon energy calibration with the ATLAS detector using LHC Run 1 data, Eur. Phys. J. C 74 (2014) 3071, arXiv: 1407.5063 [hep-ex].

[142] ATLAS Collaboration, Jet energy measurement with the ATLAS detector in proton-proton collisions at $\sqrt{s}=7 \mathrm{TeV}$, Eur. Phys. J. C 73 (2013) 2304, arXiv:1112.6426 [hep-ex].

[143] ATLAS Collaboration, Jet Calibration and Systematic Uncertainties for Jets Reconstructed in the ATLAS Detector at $\sqrt{s}=13 \mathrm{TeV}$, ATL-PHYS-PUB-2015-015, 2015. https://cds . cern.ch/record/2037613.

[144] ATLAS and CMS Collaborations, Combined Measurement of the Higgs Boson Mass in pp Collisions at $\sqrt{s}=7$ and 8 TeV with the ATLAS and CMS Experiments, Phys. Rev. Lett. 114 (2015) 191803, arXiv:1503.07589 [hep-ex].

[145] R. Boughezal, X. Liu, F. Petriello, F. J. Tackmann, and J. R. Walsh, Combining Resummed Higgs Predictions Across Jet Bins, Phys. Rev. D89 (2014) no. 7, 074044, arXiv:1312.4535 [hep-ph]. 
[146] E. Laenen, Resummation, https://www.nikhef.nl/pub/theory/center-talks/ ericlaenen_14-9-07.pdf, Sept, 2007.

[147] S. Dulat, T.-J. Hou, J. Gao, M. Guzzi, J. Huston, P. Nadolsky, J. Pumplin, C. Schmidt, D. Stump, and C. P. Yuan, New parton distribution functions from a global analysis of quantum chromodynamics, Phys. Rev. D93 (2016) no. 3, 033006, arXiv:1506.07443 [hep-ph].

[148] L. A. Harland-Lang, A. D. Martin, P. Motylinski, and R. S. Thorne, Parton distributions in the LHC era: MMHT 2014 PDFs, Eur. Phys. J. C75 (2015) no. 5, 204, arXiv:1412.3989 [hep-ph].

[149] ATLAS Collaboration, Constraints on non-Standard Model Higgs boson interactions in an effective Lagrangian using differential cross sections measured in the $H \rightarrow \gamma \gamma$ decay channel at $\sqrt{s}=8 \mathrm{TeV}$ with the ATLAS detector, Physics Letters B 753 (2016) 69 - 85.

[150] ATLAS Collaboration, Measurements of fiducial and differential cross sections for Higgs boson production in the diphoton decay channel at $\sqrt{s}=8$ TeV with ATLAS, JHEP 09 (2014) 112, arXiv:1407.4222 [hep-ex] .

[151] ATLAS Collaboration, Fiducial and differential cross sections of Higgs boson production measured in the four-lepton decay channel in pp collisions at $\sqrt{s}=8$ TeV with the ATLAS detector, Phys. Lett. B738 (2014) 234-253, arXiv: 1408.3226 [hep-ex] . 
[152] ATLAS Collaboration Collaboration, Measurements of Higgs boson properties in the diphoton decay channel with $36.1 \mathrm{fb} b^{1} \mathrm{pp}$ collision data at the center-of-mass energy of $13 \mathrm{TeV}$ with the ATLAS detector, ATLAS-CONF-2017-045, CERN, Geneva, Jul, 2017.

https://cds. cern.ch/record/2273852.

[153] ATLAS Collaboration, ATLAS Phase-II Upgrade Scoping Document, CERN-LHCC-2015-020. LHCC-G-166, CERN, Geneva, Sep, 2015. https://cds.cern.ch/record/2055248.

[154] ECFA High Luminosity LHC Experiments Workshop: Physics and Technology Challenges. 94th Plenary ECFA meeting, ECFA-13-284, CERN, Geneva, 2013. https://cds.cern.ch/record/1631032.

[155] ECFA High Luminosity LHC Experiments Workshop - Physics and Technology Developments Summary submitted to ECFA2014. 96th Plenary ECFA meeting, ECFA-15-289, CERN, Geneva, 2014. https://cds. cern. ch/record/1983664. 